\title{
Intracochlear electrical stimulation to suppress tinnitus
}

Citation for published version (APA):

Arts, R. A. G. J. (2016). Intracochlear electrical stimulation to suppress tinnitus. [Doctoral Thesis, Maastricht University]. Maastricht University. https://doi.org/10.26481/dis.20160115ra

Document status and date:

Published: 01/01/2016

DOI:

10.26481/dis.20160115ra

Document Version:

Publisher's PDF, also known as Version of record

\section{Please check the document version of this publication:}

- A submitted manuscript is the version of the article upon submission and before peer-review. There can be important differences between the submitted version and the official published version of record.

People interested in the research are advised to contact the author for the final version of the publication, or visit the DOI to the publisher's website.

- The final author version and the galley proof are versions of the publication after peer review.

- The final published version features the final layout of the paper including the volume, issue and page numbers.

Link to publication

\footnotetext{
General rights rights.

- You may freely distribute the URL identifying the publication in the public portal. please follow below link for the End User Agreement:

www.umlib.nl/taverne-license

Take down policy

If you believe that this document breaches copyright please contact us at:

repository@maastrichtuniversity.nl

providing details and we will investigate your claim.
}

Copyright and moral rights for the publications made accessible in the public portal are retained by the authors and/or other copyright owners and it is a condition of accessing publications that users recognise and abide by the legal requirements associated with these

- Users may download and print one copy of any publication from the public portal for the purpose of private study or research.

- You may not further distribute the material or use it for any profit-making activity or commercial gain

If the publication is distributed under the terms of Article $25 \mathrm{fa}$ of the Dutch Copyright Act, indicated by the "Taverne" license above, 


\section{Intracochlear electrical stimulation to suppress tinnitus}

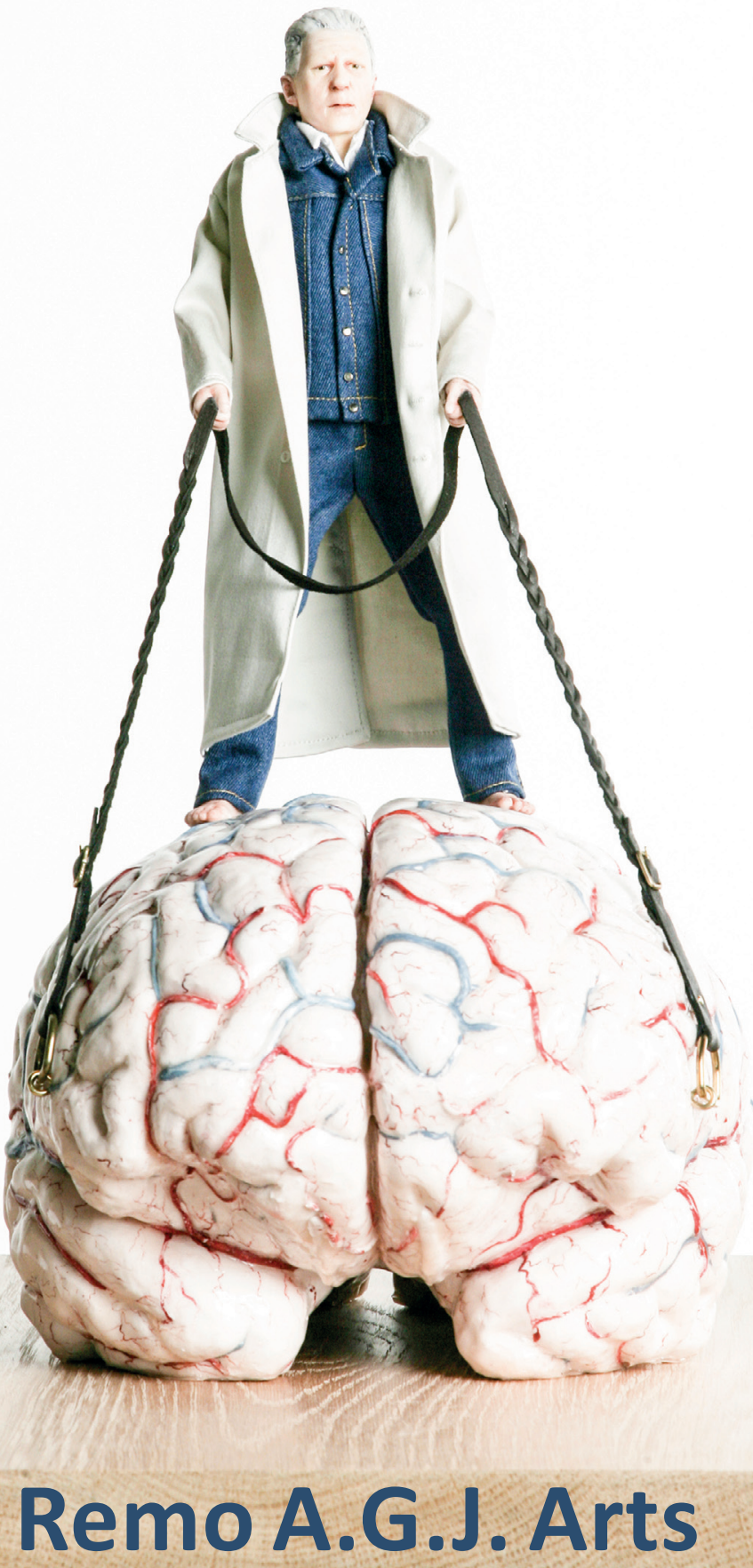





\section{Intracochlear electrical stimulation}

to suppress tinnitus

\section{Copyright Remo Arts, Maastricht 2015}

Cover design (front):

$$
\text { Jan Fabre }
$$

The artist who tries to drive his own brain forward (2007)

Material: $\quad$ sillicones, textiles, oak and paint

Size: $\quad 23 \times 32 \times 43.5 \mathrm{~cm}$

Installation view: Jan Fabre. Antropologia di un pianeta. (Exh. Cat.)

2007 Venezia, Palazzo Benzon (Oct. 2007)

Photographer: Pat Verbruggen

Copyright: Angelos bvba

Medium: sculpture

Cover design (back):

$$
\text { Matt Might }
$$

The Illustrated Guide to a Ph.D.

Source: $\quad$ http://matt.might.net/articles/phd-school-in-pictures/

Matt Might, a professor in Computer Science at the University of Utah

created The Illustrated Guide to a Ph.D. to explain what a Ph.D. is. [Matt has

licensed the guide for sharing with special terms under the Creative

Commons license.]

Layout:

De Weijer Design BNO

Production:

CPI Wöhrmann Print Service B.V.

ISBN:

978-94-6203-971-1

Financial support for the publication of this thesis was kindly provided by:

ABN AMRO Bank N.V., Advanced Bionics Benelux B.V., Beter Horen B.V., ZEISS, Cochlear

Benelux NV, Daleco Pharma B.V., Electro Medical Instruments Doesberg B.V., KNO-vereniging,

Laservision Instruments B.V., MED-ELDeutschland GmbH, Meditop Medical Products B.V.,

Olympus Nederland B.V., Oticon Medical, Pentax Medical, Sonova Nederland B.V., Specsavers,

Universiteit Maastricht

\section{PROEFSCHRIFT}

ter verkrijging van de graad van doctor aan de Universiteit Maastricht

op gezag van de Rector Magnificus, Prof. dr. L.L.G. Soete

volgens het besluit van het College van Decanen,

in het openbaar te verdedigen op vrijdag 15 januari 2016, om 14:00 uur. door

Remo Albert Gerardus Joseph Arts 
Prof. dr. R.J. Stokroos

\section{Co-promotor}

Dr. E.L.J. George

\section{Beo ordelingscommissie}

Prof. dr. B. Kremer (voorzitter)

Prof. dr. P. van Dijk (Rijksuniversiteit Groningen, NL)

Prof. dr. W.H. Mess

Prof. dr. Y. Temel

Dr. K. Vermeire (Long Island Jewish Medical Center, NY, USA)
Chapter 1 Review: cochlear implants as a treatment of tinnitus in single sided deafness

Current Opinion in Otolaryngology \& Head and Neck Surgery 2012;20:398-403

Chapter 2 The occurrence of tinnitus after $\mathrm{Cl}$ surgery in patients with severe hearing loss: A retrospective study

Int J Audiol 2015;Sep24:1-8

Chapter 3 Optimizing intracochlear electrical stimulation to suppress tinnitus

Ear \& Hearing 2015;36:125-135

Chapter 4 Long-term effects of intracochlear electrical stimulation with looped patterns on tinnitus: a case study

Accepted by Ear, Nose \& Throat Journal

Chapter 5 Tinnitus suppression by intracochlear electrical stimulation in single sided deafness - a prospective clinical trial: Part

Audiology and Neurotology 2015;20:294-313

Chapter 6 Tinnitus suppression by intracochlear electrical stimulation in single sided deafness - a prospective clinical trial: Part II

Submitted for publication

Chapter 7 Hearing after cochlear implantation in patients with unilateral severe to profound hearing loss accompanied with tinnitus and the effect of formal auditory training.

Submitted for publication

Summary \& General discussion

Samenvatting

Dankwoord

Curriculum Vitae

List of publications 
Tinnitus (aurium), meaning ringing of the ears is the meaningless phantom sensation of sound, affectin about 50 million people in the United States and an estimated 70 million in the European Union [Cederroth et al., 2013]. The estimated tinnitus prevalence in the Netherlands is 2 million of which about 60.000 people severely suffer from this disorder [Cima et al. 2009]. Some of these severe sufferers experience serious problems in daily life and even consider suicide [Turner et al., 2007].

Furthermore, based on the data of 2009 [Maes et al., 2013], the economic burden of tinnitus to society is substantial with an average annual tinnitus related societal cost per patient of $€ 5,315$ in the Netherlands. Most of these costs were associated with production losses; on average $€ 3,702$. The annual health care costs per patient was estimated to be $€ 1,544$ on average. Overall, the annual costs of tinnitus in the Netherlands examined from a societal perspective is about $€ 6.8$ billion ( $95 \%$ confidence interval: $€ 3.9$ billion - $€ 10.8$ billion). The majority cost spent on this illness was not healthcare related. The annua health care costs were $€ 1.9$ billion ( $95 \%$ confidence interval: $€ 1.4$ billion - $€ 2.5$ billion). This amounts to $2.3 \%$ of the total Dutch health care expenditure.

Tinnitus is characterized as the meaningless phantom sensation of sound and can be distinguished from auditory hallucinations. Auditory hallucinations are characterized by meaningful sounds such as speech, which can occur in, for instance, schizophrenia [Møller et al., 2011]. Unfortunately, tinnitus proved extremely difficult to treat while the incidence is expected to increase over time due to modern livin conditions [Gilles et al., 2013]. To date, tinnitus remains refractory to current medical treatment and therefore only management treatments and therapies can be offered [Jastreboff 1999; Cima et al. 2012]

The difficulty of treating tinnitus is probably partly the result of the heterogeneity in the underlying cause of this disorder. Tinnitus can for example be divided into objective and subjective tinnitus. Objective innitus, accounting for about $1 \%$ of all tinnitus cases, arises from an internal physical source (e.g. cochleovestibular neurovascular conflicts, muscle contractions and temporomandibular joint disorders) and is in general pulsatile [Hall et al., 2011]. The major category, subjective tinnitus, has no internal physical source and is audible only to the patient. This doctoral dissertation is focussed on subjective tinnitus. Thus, whenever the term 'tinnitus' is used, we refer to its subjective type, except when explicitly indicated.

Through the centuries, different ideas arose about the underlying cause of tinnitus and related to thes ideas, different treatment options have been considered. The old Egyptians believed that tinnitus occured from a bewitched ear (Figure 1) and they infused oils or herbs in the outer ear canal as a treatment option [Stephens, 1984; EUtinnitus]. Later, it was believed that the ear itself was the tinnitus cause. Some historians believe that Vincent Van Gogh (1853-90) was forced to cut off his right ear in 1888 in order to get relief from his troublesome tinnitus (Figure 2) [Bhattacharyya and Rai, 2015]

\section{If

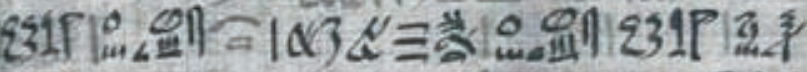

Figure 1: Item number 768 of the Egyptian papyrus Ebers. Ebbell (1937) translated the first part of this section as 'the bewitched ear', the second part was translated as 'pus in the ear'. With permission from Dr. E. Ooms from the university of Ghent.
Recently tinnitus is expected to have a more central origin. The current consensus is that tinnitus is the result of maladaptive plasticity in the central auditory pathway as a result of auditory deprivation [D Verney, 1731; Engineer et al., 2011]. Although the exact cause of tinnitus is still unknown, several mechanisms underlying tinnitus have been suggested. Some of these are changes in central gain to compensate for hearing loss (Figure 3 ), increased spontaneous activity and synchrony of cortical neuron by tonotopic reorganization (Figure 4) and a shift in the balance of excitatory and inhibitory neurotransmitters or its receptors (Figure 5) [Eggermont and Roberts, 2004, 2012; Schaette and McAlpine, 2011; Noreña and Farley, 2013; Roberts et al., 2013].

Based on these mechanisms of neural plasticity as the underlying cause, tinnitus should be reversible by ncreasing auditory stimulation, known as the 'bottom-up approach'. Acoustic sound enrichment by either conventional hearing aids [Saltzman and Ersner, 1947; Shekhawat et al., 2013; Hoare et al., 2014] tinnitus maskers [Erlandsson et al., 1987; Vernon and Meikle, 2003; Fioretti et al., 2012] or music with tinnitus-related modification [Davis et al., 2007; Tass et al., 2012; Pantev et al., 2012a; Pantev et al., 2012b; Reavis et al., 2012] is a potential treatment option, but shows a large variability in effectivenes among patients. This variability may be explained by the heterogeneity of tinnitus, which seems to be caused by auditory deprivation and can occur at various positions along the auditory pathway. As the most common cause of auditory deprivation is in the cochlea at the level of the hair cells, treatment options need to bypass this pathology.

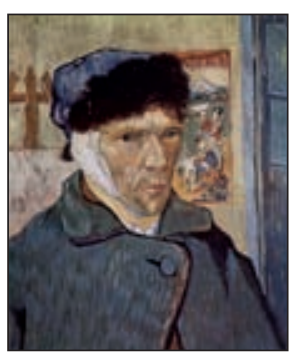

gigure 2: Self-Portrait with Bandaged Ear, Vincent Van Gogh, 1889, oil on canvas, 60x49cm (Courtauld Galleries, London).

In 1748, Giuseppe Veratti (1707-93) followed by Alessandro Volta (1745-1827) in 1800 were the firs individuals who reported about electrical stimulation of the auditory nerve and the induced sensations. These findings resulted, after intensive research of, inter alia, Lundberg, Djourno, Eyries, Doyle, House, Michelson and Clark in a formal Food and Drugs Administration (FDA) approval of the "3M/House" cochlear implant (Cl) in November 1984 and the introduction of the first multiple channel device under the name "Nucleus Multi-channel Cochlear Implant" [Biomed.brown.edu].

$\mathrm{ACl}$ is an electronic medical device, partly implanted in the cochlea, that transforms acoustic sounds into electrical current that directly stimulates the auditory nerve. This device has been developed for patients suffering from bilateral severe to profound sensorineural hearing loss for whom amplification of sounds with conventional hearing aids does not sufficiently restore the ability to understand speech. In these patients, the auditory nerves that lead to the brain cannot be stimulated as a consequence of loss of hair cells. The $\mathrm{Cl}$ was therefore developed to bypass the deficient transducer structures and produces action potentials at the auditory nerve sites (or the residual neurons, depending on the degree and type of pathology) using direct electrical stimulation to facilitate delivery of information to the primary and secondary auditory cortex. 


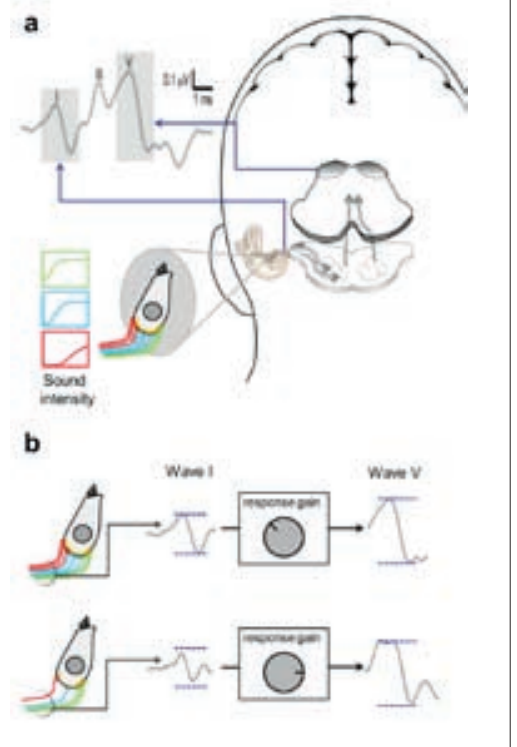

Figure 3
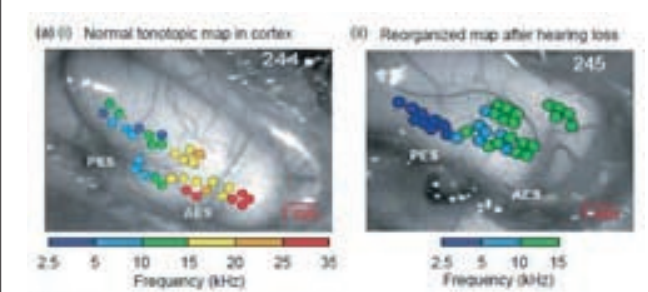

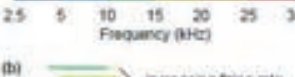

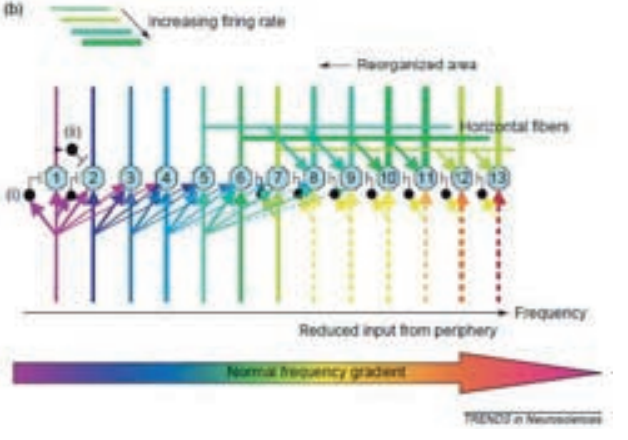

Figure 4

Figure 3: Auditory brainstem responses, hidden hearing loss, and homeostatic gain control in the auditory system. a Illustration of the genertion sites of wave I (auditory nerve) and wave V (midbrain) of the ABR, schematic depiction of an inner hair cell of the cochlea and of the AN fibers contacting it, and the rate-versus-intensity functions of the different types of auditory nerve fibers (green, low threshold fibers; blue, medium threshold fibers: red, high threshold fibers) $b$, Illustration of how homeostatic gain control in the auditory brainstem could normalize wave Vamplitude after hidden hearing loss. In the healthy situation (top) a complete population of AN fibers gives rise to a full-sized ABR wavel, response ain in the brainstem is low, and wave Vhas anormal amplitude. In hidden hearing loss (bottom a fraction of the AN fibers no longer responds to sound, leading to a redude.

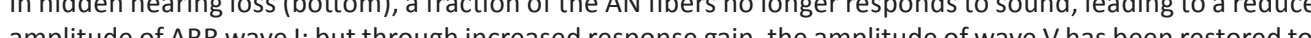
With permission from Dr. R. Schaette London.

Figure 4: Normal and reorganized tonotopic maps in primary auditory cortex (AI). (a) The characteristic frequency at each recording site is color-coded and overlaid on a photograph of the cortical surface for a control cat (i) and a cat with a noise induced hearing loss (ii). The hearing loss was limited to frequencies $>10 \mathrm{kHz}$ and amounted to $3 \mathrm{~dB}$ at $12 \mathrm{kHz}, 12 \mathrm{~dB}$ at $16 \mathrm{kHz}, 22 \mathrm{~dB}$ at $24 \mathrm{kHz}$ and $23 \mathrm{~dB}$ at $32 \mathrm{kHz}$. (244 and 245 are cat identification numbers.) (b) The effect of restricted high-frequency hearing loss on the input to pyramidal cells (numbered 1-13) in auditory cortex The large colored arrow shows the normal frequency gradient of the inputs conveying the tonotopic mapping. The thin vertical lines leading to the cortical cells are color-coded to reflect their frequency-specific input from the thalamus. For the higher frequencies, covering the range of the hearing loss, the lines are shown as dashed to indicate their reduced ability to activate cortical cells at low stimulus levels and during silence. Numerous divergen connections lead from each thalamic cell to a range of cortical cells (indicated by lines with the same color). A few inhibitory feedforward connections are indicated [one is labeled (i) on the left]. These affect the same cells that receive their thalamic inputs. Feedback inhibition is also prevalent but is only shown for cell 1 (ii). The assumption is that loss of input limits not only the excitation but also, even more strongly, the inhibitory feedforward activity. As a result, the diverging thalamic inputs from neighboring unaffected cells, and the inputs from cortical cells via horizontal fibers, face less competition from inhibition at those cortical cells deprived from thalamic input. Thus, these excitatory inputs are disinhibited or 'unmasked' and can impose their own frequency-selective inputs on cortical cells in the hearing loss range, which will ultimately result in a reorganization of the tonotopic map in the hearing-loss animal. Abbreviations: AES, anterior ectosylvian sulcus; PES, posterior ectosylvian sulcus [Eggermon and Roberts, 2004]. With permission from Prof. Emer. J. Eggermont from the university of Galgary.

This medical device, implanted in over 300,000 people worldwide up to 2012 [National Institute on Deafness and Other Communication Disorders], consists of an external and internal part (Figure 6). The external part includes a microphone to detect acoustic sounds, a processor which selectively filters sounds by splitting the sound into channels and sends these signals to the transmitter. The transmitter is positioned outside the head at the height of the internal receiver, using a magnet. Moreover, the external part includes a battery pack and an optional remote control. The external transmitter is coupled via a radio-frequency link to the internal receiver. Of the internal part, the receiver is surgically placed behind the ear underneath the skin and the electrode array is implanted into to cochlea. From the receiver, the decoded information is transmitted to the electrodes on the electrode array. Electrode arrays may comprise of 12 to 22 stimulating electrodes. Roughly, each electrode represents a specific frequency band, based on the natural tonotopic organization of the cochlea while the loudness is mainly influenced by the generated charge.

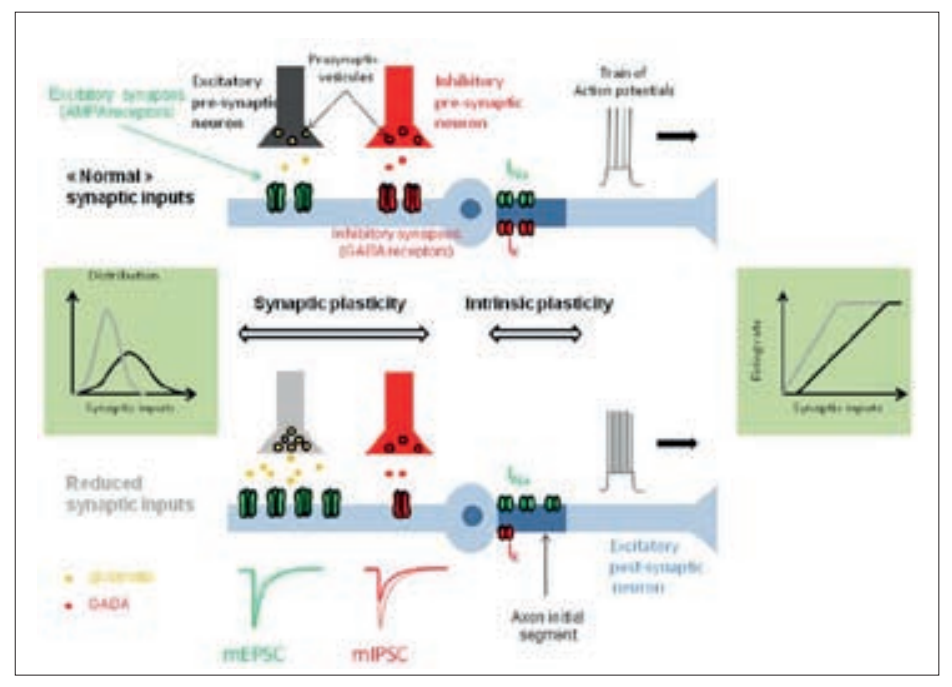

Figure 5: Basic scheme of homeostatic plasticity following activity deprivation. On the left, mechanisms related to pre- and post-synaptic homeostasis are illustrated. Activity blockade has been shown to increase alpha-amino-3hydroxy-5-methyl-4-isoxzole propionic acid (AMPA) channel expression and decrease gamma-aminobutyric acid (GABA) channel expression on postsynaptic neurons. These changes result in increased miniature excitatory postsynaptic currents (MEPSCS, before and after activity blockade, dotted and continuous line, respectively) and decreased miniature inhibitory postsynaptic currents (mIPSC). At the presynaptic level, chronic suppression of neura activity is accmpan reare probability. On the right, hom chase distribution: of synaptic inputs ar deprivit At the extreme right the firing rate of the post-synaptic nuron as a function of the synaptic inputs is depriven At the suchchangesmayalso resultinincreased spontaneous activity [Noreña and Farley, 2013] With persissionfrom A. Noreña from Aix-Marselle University.

William House reported, after some experiments of Wibel and Grapengiesser on static electricity as a therapeutic approach to tinnitus, on tinnitus suppression in one of the first $\mathrm{Cl}$ patients [House, 1976]. After decades of research it has become clear that tinnitus suppression is a common side effect of cochlear implants [Ito and Sakakihara, 1994; Aschendorff et al., 1998; House, 1999; Steenerson and 
Cronin 1999; Ruckenstein et al., 2001. Miyamoto and Bichey, 2003. Di Nardo et al., 2007: Tyler et al., 2008; Van de Heyning et al., 2008; Arndt et al., 2010; Buechner et al., 2010; Olze et al., 2011; Olze et al., 2012; De Carvalho et al., 2013]. Nevertheless, tinnitus can also be a complication after cochlear implantation [Di Nardo et al., 2007; Postelmans et al., 2007; Hou et al., 2010] which is possibly related to cochlear trauma due to the surgery [Nordfalk et al., 2014; Todt et al., 2014, 2015].
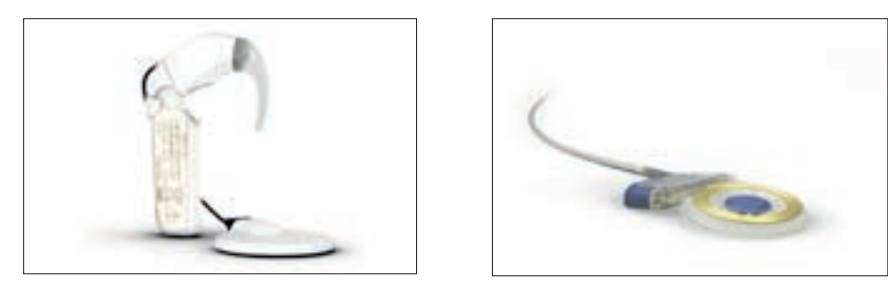

Figure 6: External (left) and internal (right) part of a Cl. Here, a MED-EL cochlear implant system (MED-EL Corporation, Innsbruck, Austria) is shown, consisting of the OPUS2 speech processor and the CONCERTO implant.

\section{Aim of this thesis}

The aim of this research was to evaluate the relationship between intracochlear electrical stimulation that is generated by $\mathrm{Cl}$ and tinnitus. Furthermore, we optimize stimulation characteristics for tinnitus suppression.

The following questions were addressed:

What can be concluded, after an extensive search in the literature, about the effect of $\mathrm{Cl}$ on tinnitus in single sided deafness (SSD)?

In Chapter 1, we performed the first (scoping) review that shows an overview of all publicly available reports about $\mathrm{Cl}$ as a treatment for tinnitus in SSD. Here, nine full research articles have been reported.

Furthermore, induction of tinnitus is also a known complication of $\mathrm{Cl}$-surgery. However, the incidence varies considerably among the studies. To date, it remains unclear why some $\mathrm{Cl}$-recipients experience tinnitus complaints due to $\mathrm{Cl}$-surgery while others do not. A possible explanation is that deterioration of residual hearing as a result of the traumatic insertion of the electrode array may trigger the experience of tinnitus postoperatively. This led to the following question:

\section{What is the effect of detected deterioration of residual hearing due to $\mathrm{Cl}$-surgery on tinnitus?}

Chapter 2 describes a retrospective study using the database of the $\mathrm{Cl}$-team of South-East Netherlands, based at the Maastricht University Medical Center. Of the 512 patients who were implanted between 2003 and 2013, 247 patients met the inclusion criteria and were sent a questionnaire. Of these, 197 patients were included.

Are we able to suppress tinnitus with electrical stimulation, that does not directly encode acoustic sound, in bilateral severe to profound deaf subjects with unilateral $\mathrm{Cl}$ and tinnitus?

Since $\mathrm{Cl}$ seems to have a positive effect on tinnitus (Chapter 1 ), the question arises what mechanism is responsible for tinnitus suppression after cochlear implantation. Is this suppression due to the reversal of the assumed maladaptive plasticity or is it simply the shift in attention from the tinnitus to environmental sound and therefore less awareness that reduces tinnitus perception?
In Chapter 3 , a prospective clinical trial is reported about the short-term effects on tinnitus in a cohort of eleven subjects with bilateral severe to profound hearing loss, unilateral $\mathrm{Cl}$ and tinnitus.

What are the long-term effects of both standard clinical $\mathrm{Cl}$ and intracochlear electrical stimulation independent of an acoustic input on tinnitus in a single sided deaf subject with unilateral tinnitus? In order to investigate the long-term effects of intracochlear electrical stimulation independent of acoustic input, a contralateral normal hearing ear is required. This contralateral ear enables the communication during intracochlear electrical stimulation independent of acoustic sound.

Chapter 4 reports about a case presented with a two-year history of debilitating tinnitus in the right ear following an idiopathic profound sudden sensorineural hearing loss in the right ear with a Pure Tone Average (PTA; averaged across $0.5,1$ and $2 \mathrm{kHz}$ ) of $97 \mathrm{~dB}$ hearing level (HL). His right ear was implanted with a $\mathrm{Cl}$ in order to suppress his tinnitus. The standard clinical $\mathrm{Cl}$ rehabilitation was followed by a threemonth period of looped intracochlear electrical stimulation (without environmental sound perception) Although the results of Chapter 4 are promising, the evidence of this finding is limited since a single case is reported. Therefore, a cohort of ten single sided deaf subjects with tinnitus localized to the deaf ear is described in Chapter 5-7. In these chapters, the following questions were answered:

What are the optimal stimulation characteristics for short-term tinnitus suppression in SSD with electrical stimulation independent of acoustic sound?

Chapter 5 describes a prospective clinical trial about the short-term effects of looped electrical stimulation on tinnitus (without environmental sound perception). Furthermore, with the proposed study design we were able to answer three additional research questions: 1 . Does the surgery itself suppress tinnitus? 2 . Does the clinical $\mathrm{Cl}$ suppress tinnitus? and 3 . Is a one-week period of $\mathrm{Cl}$-deactivation sufficient to rule out the possible influence of providing a hearing sensation on the experience of tinnitus?

\section{What are the long-term effects of intracochlear electrical stimulation, that does not encode} environmental sounds, on tinnitus?

The main objective of Chapter 6 is to investigate the possibility for the development of a "Tinnitus Implant" (TI), an intracochlear pulse generator for the suppression of tinnitus. Although the results obtained in Chapter 5 seem to be promising, long-term tinnitus suppression is a requirement for the viability of the TI. Therefore, long-term suppressive effects of looped electrical stimulation (without environmental sound perception) were compared with the standard stimulation pattern of a $\mathrm{Cl}$ (with environmental sound perception).

Finally, one last relevant research question remains unanswered, namely:

What is the effect of intracochlear electrical stimulation that does not encode environmental sounds, but suppresses tinnitus, on speech perception in SSD?

In Chapter 7, the effects of looped electrical stimulation on speech perception are reported. Furthermore, we aim to answer two additional research questions: 1 . What is the effect of the standard clinical $\mathrm{Cl}$ on speech perception in SSD? and 2. Is formal auditory training beneficial during $\mathrm{Cl}$-rehabilitation in SSD? 


\section{References}

Arndt S, Aschendorff A, Laszig R, Beck R, Schild C, Kroeger S, Ihorst G, Wesarg T: Comparison of pseudobinaural hearing to real binaural hearing rehabilitation after cochlear implantation in patients with unilateral deafness and tinnitus. Otol Neurotol 2010;32:39-47.

Aschendorff A, Pabst G, Klenzner T, Laszig R: Tinnitus in cochlear implant users: The Freibur g Experience. Int Tinnitus J 1998;2:162-164.

Bhattacharyya KB, Rai S: The neuropsychiatric ailment of Vincent Van Gogh. Ann Indian Acad Neurol 2015; 18(1):6-9.

Biomed.brown.edu. History of Cochlear Implants. Available at: http://biomed.brown.edu/Courses/ BI108/BI108_2001_Groups/Cochlear_Implants/history.html. Accessed: May 12, 2015.

Buechner A, Brendel M, Lesinski-Schiedat A, Wenzel G, Frohne-Buechner C, Jaeger B, Lenarz T: Cochlear implantation in unilateral deaf subjects associated with ipsilateral tinnitus. Otol Neurotol 2010;31:1381 1385.

Cederroth CR, Canlon B, Langguth B: Hearing loss and tinnitus - are funders and industry listening? Nature Biotechnology 2013; 31:972-4.

Cima RF, Joore MA, Maes IH, Scheyen DJ, El Refaie A, Baguley DM, Vlaeyen JW, Anteunis LJ: Costeffectiveness of multidisciplinary management of tinnitus at a specialized Tinnitus centre. BMC Health Serv Res 2009;11;9:29

Cima RF, Maes IH, Joore MA, Scheyen DJ, El Refaie A, Baguley DM, Anteunis LJ, van Breukelen GJ, Vlaeyen $\mathrm{JW}$ : Specialised treatment based on cognitive behaviour therapy versus usual care for tinnitus: randomized controlled trial. Lancet 2012;379(9830):1951-9.

Davis PB, Paki B, Hanley PJ: Neuromonics tinnitus treatment: third clinical trial. Ear Hear 2007;28:242

De Carvalho GM, Guimaraes AC, Duarte ASM, Muranaka EB, Soki MN, Martins RSZ, Bianchini WA, Paschoal JR, Castilho AM: Hearing preservation after cochlear implantation: UNICAMP outcomes. Int J Otolaryngol DOI: 10.1155/2013/107186.

Di Nardo W, Cantore I, Cianfrone F, Melillo P, Scorpecci A, Paludetti G: Tinnitus modifications after cochlear implantation. Eur Arch Otorhinolaryngol 2007;264:1145-1149.

Du Verney G: Traité de l'organe de l'ouïe: contentant la structure, les usages \& les maladies de toutes les parties de l'oreille (1731). Leide: Joh. Arn. Langerak.

Eggermont JJ, Roberts LE: The neuroscience of tinnitus. TRENDS in Neurosciences 2004; 27(11):676-
Eggermont JJ, Roberts LE: The neuroscience of tinnitus: understanding abnormal and norma perception. Front Syst Neurosci DOI: 10.3389/2012/00053.

Engineer ND, Riley JR, Seale JD, Vrana WA, Shetake JA, Sudanagunta SP, Borland MS, Kilgard MP: Reversing pathological neural activity using targeted plasticity. Nature 2011;470(7332):101-104.

Erlandsson S, Ringdahl A, Hutchins T, Carlsson SG: Treatment of tinnitus: a controlled comparison of masking and placebo. Br J Audiol 1987;21(2):37-44.

EUtinnitus. History of tinnitus. Available at: http://www.eutinnitus.com/downloads/History\%20of\%20 Tinnitus.pdf. Accessed: May 12, 2015.

Fioretti A, Peri G, Eibenstein A: Suppression of tinnitus in a patient with unilateral sudden hearing loss: a case report Case Reports in Otolaryngology DOI:10.1155/2012/210707.

Gilles A, Van Hal G, De Ridder D, Wouters K, Van de Heyning P: Epidemiology of noise-induced tinnitus and the attitudes and beliefs towards noise and hearing protection in adolescence. PLOS ONE DOI: 10.1371/journal.pone.0070297.

Hall DA, Láinez MJA, Newman CW, Sanchez TG, Egler M, Tennigkeit F, Koch M, Langguth B. Treatment options for subjective tinnitus: self reports from a sample of general practitioners and ENT physician within Europe and the USA. BMC Health Serv Res 2011:11:302.

Hoare DJ, Edmondson-Jones M, Sereda M, Akeroyd MA, Hall D: Amplification with hearing aids for patients with tinnitus and co-existing hearing loss. Cochrane Database of Systematic Reviews DOI: 10.1002/14651858.CD010151.pub2.

Hou J-H, Zhao S-P, Ning F, Rao S-Q, Han D-Y: Postoperative complications in patients with cochlear implants and implact of nursing intervention. Acta Oto-Laryngol 2010;130:687-695.

House D: Tinnitus suppression via cochlear implants: review and remarks. Int Tinnitus J 1999;5(1):27-29

House WF: Cochlear implants. Ann Otol Rhinol Laryngol 1976;85(27):1-93.

Ito J, Sakakihara J: Suppression of tinnitus by cochlear implantation. Am J Otolaryngol 1994;15(2):145148

Jastreboff PJ: Tinnitus retraining therapy. Br J Audiol 1999;33(1):68-70.

Maes IHL, Cima RFF, Vlaeyen JW, Anteunis LJC, Joore MA: Tinnitus: a cost study. Ear Hea 2013;34(4);508-514.

Miyamoto RT, Bichey BG: Cochlear implantation for tinnitus suppression. Otolaryngol Clin N Am 2003; 36:345-352.

Møller AR (2011) Introduction. In A.R. Møller, B. Langguth, D. De Ridder, T. Kleinjung (Eds). Textbook of tinnitus (pp,3-8). New York: Springer. 
National Institute on Deafness and Other Communication Disorders, National Institute of Health. Cochlear implants. Available at: http://www.nidcd.nih.gov/health/hearing/pages/coch.aspx. Accessed: April 8, 2015.

Nordfalk KF, Rasmussen K, Hopp E, Greisiger R, Jablonski GE: Scalar position in cochlear implant surgery and outcome in residual hearing and the vestibular system. Int J Audiol 2014;53:121-127.

Noreña AJ, Farley BJ: Tinnitus-related neural activity: theories of generation, propagation, and centralization. Hearing Res 2013;295:161-171.

Olze H, Szczepek AJ, Haupt H, Förster U, Zirke N, Gräbel S, Mazurek B: Cochlear implantation has a positive influence on quality of life, tinnitus, and psychological comorbidity. Laryngoscope 2011;121:2220-2227.

Olze H, Szczepek A, Haupt H, Zirke N, Graebel ., Mazurek B: The impact of cochlear implantation on tinnitus, stress and quality of life in postlingually deafened patients. Audiol Neurotol 2012;17:2-11.

Pantev C, Okamoto $\mathrm{H}$, Teismann H: Music-induced cortical plasticity and lateral inhibition in the human auditory auditory cortex as foundations for tonal tinnitus treatment. Front Syst Neurosci DOI:

$10.3389 / 2012 / 00050$

Pantev C, Okamoto H, Teismann H: Tinnitus: the dark side of auditory cortex plasticity. Ann NY Acad Sci 2012;1252:253-258.

Postelmans JTF, Cleffken B, Stokroos RJ: Post-operative complications of cochlear implantation in adults and childeren: five years' experience in Maastricht. J Laryngol Otol 2007;121:318-323.

Reavis KM, Rothholtz VS, Tang Q, Carroll JA, Djalilian H, Zeng F-G: Temporary suppression of tinnitus by modulated sounds. J Assoc Res Otolaryngol 2012;13(4):561-71.

Roberts LE, Husain FT, Eggermont JJ: Role of attention in the generation and modulation of tinnitus. Neurosci Biobehav Rev 2013;37(8):1754-73

Ruckenstein MJ, Hedgepeth C, Rafter KO, Montes ML, Bigelow DC: Tinnitus suppression in patients with cochlear implants. Otol Neurotol 2001;22:200-204.

Saltzman M, Ersner M: A hearing aid for the relief of tinnitus aurium. Laryngoscope 1947;57:358-66.

Schaette R, McAlpine D: Tinnitus with a normal audiogram: physiological evidence for hidden hearing loss and computational model. J Neurosci 2011;31(38):13452-13457.

Shekhawat GS, Searchfield GD, Stinear CM: Role of hearing aids in tinnitus intervention: a scoping review. J Am Acad Audiol 2013;24:747-762.

Steenerson RL, Cronin GW: Treatment of tinnitus with electrical stimulation. Otolaryng Head Neck 1999;121:511-513
Stephens SDG: The treatment of tinnitus - a historical perspective J Jaryngol Otol 1984:98:963-972.

Tass PA, Adamchic I, Freund H-J, von Stackelberg T, Hauptmann C: Counteracting tinnitus by acoustic coordinated reset neuromodulation. Restor Neurol Neurosci 2012;30:137-159.

Todt I, Rademacher G, Mutze S, Ernst A: Scalar electrode position and the occurrence of tinnitus in cochlear implantees. XI International Tinnitus Seminar, Berlin 2014.

Todt I, Rademacher G, Mutze S, Ramalingam R, Wolter S, Mittmann P, Wagner J, Ernst A: Relationship between intracochlear electrode position and tinnitus in cochlear implantees. Acta Oto-Laryngologica 2015;26:1-5

Turner O, Windfuhr K, Kapur N: Suicide in deaf populations: a literature review. Ann Gen Psychiatry 2007:6:26.

Tyler RS, Rubinstein J, Pan T, Chang S-A, Gogel SA, Gehringer A, Coelho C: Electrical stimulation of the cochlea to reduce tinnitus. Semin Hear 2008;29(4):326-332.

Van de Heyning P, Vermeire K, Diebl M, Nopp P, Anderson I, De Ridder D: Incapacitating unilatera tinnitus in single-sided deafness treated by cochlear implantation. Ann Oto Rhinol Laryn 2008;117(9):645-652.

Vernon JA, Meikle MB: Masking devices and alprazolam treatment for tinnitus, Otolaryngol Clin N Am 2003;36:307-320. 


\section{Chapter}

Review: cochlear implants as a treatment of tinnitus in single sided deafness.

Remo A.G.J. Arts M.Sc,

Erwin L.J. George Ph.D,

Robert J. Stokroos MD, Ph.D

and Katrien Vermeire Ph.D

Department of ENT/Head and Neck Surgery,

Maastricht University Medical Centre,

Maastricht, The Netherlands

Curr Opin Otolaryngol Head Neck

Surg 2012;20:398-403 DOI:10.1097/

MOO.0b013e3283577b66 
Abstract:

Purpose of review: Tinnitus is a symptom that is highly associated with hearing loss. Its incidence is expected to increase, due to the detrimental effects of occupational and leisure noise. Even though no standard treatment is currently available, the effect of cochlear implants (CI) on tinnitus in Single-Sided Deafness (SSD) is under scientific attention. This review reveals an overview of all public available reports about $\mathrm{Cl}$ as a treatment for tinnitus in SSD.

Recent findings: Cochlear implantation in SSD suppresses tinnitus in most of the cases. Some studies even demonstrate complete tinnitus suppression after implantation. No tinnitus worsening is reported in any of the cases. Furthermore, tinnitus does not restore during the electrical stimulation presented by the $\mathrm{Cl}$. The experienced tinnitus level seems to stabilize after 3 to 6 months after the first fitting.

Summary: Although the underlying mechanism responsible for the observed tinnitus suppression is not yet clear, cochlear implantation should be considered as a treatment option for tinnitus arising from single sided deafness. However, appropriate patient selection is essential as it is expected that it is a requirement that tinnitus arises from cochlear deafferentiation.

Key words: Tinnitus; Single Sided Deafness; Cochlear Implants

\section{Introduction:}

Tinnitus can be defined as an otologic complaint, arising from an experienced auditory phantom sensation in the absence of an external physical source. Tinnitus is generally divided into two categories: objective and subjective. Objective tinnitus arises from an internal physical source (e.g. cochleovestibular neurovascular conflict) and is in general pulsatile[1]. Only a minor $1 \%$ of the tinnitus cases is objective; that's why objective tinnitus is disregarded in this review. The major category, subjective tinnitus, is audible only to the patient and occurs at different levels of severity. The underlying pathophysiology remains as yet unclear[1].

Although there is still discussion concerning the cause of tinnitus, it is hypothesized that tinnitus arises from changes in neural activity caused by a reduced (or lost) auditory input, for instance due to hearing loss [2, 3]. Prior animal studies seem to give evidence for cortical reorganization of the tonotopic map[3]. Unfortunately, it is still unclear whether tinnitus arises from this cortical reorganization or from the confounding effect of the hearing loss. Therefore, recently a study was performed with normal-hearing patients with and without tinnitus[4]. No evidence was found for any cortical reorganization of the tonotopic map as the underlying mechanism of tinnitus, at least not at the macroscopic level[4].

Another well-known hypothesis for the underlying mechanism of tinnitus is related to the first-mentioned. Here, an increased neural activity in the central auditory areas as a result of an imbalance of excitatory and inhibitory inputs conveyed to central auditory structures due to a hearing loss, causes tinnitus[3]. However animal studies seem to give evidence for this hypothesis, it can again not be excluded that hearing loss is a confounder.

Subjective tinnitus occurs in 10 to $15 \%$ of the general population, where 1 to $3 \%$ has severe distressing tinnitus[5]. It may have a major impact on daily life[5] and its incidence is expected to increase due to occupational and leisure noise.

Although the pathophysiologic mechanism underlying tinnitus still remains unclear, it is striking that $85 \%$ of tinnitus cases are accompanied by hearing loss. Tinnitus is therefore more common in adults, especially olderadults [1]. Furthermore, in adult patients with bilateral deafness, that undergo cochlear implantation for the classical indication of hearing restoration, tinnitus occurs in $66 \%$ to $86 \%$ of the cases before implantation[6]. The prevalence of tinnitus in Single Sided Deaf (SSD) patients is unknown, but it is expected that it is comparable to the prevalence of tinnitus in bilateral deaf patients.

Most current therapies for tinnitus consist of cognitive behavioral treatment in combination with sound enrichment $[7,8]$. These treatments aim at improving habituation and coping strategies and are generally based on Jastreboffs neurophysiological model[9]. Conventional hearing aids, distraction sounds and tinnitus maskers are all forms of sound enrichment which are used as a treatment for tinnitus.

However, sound enrichment becomes problematic in patients that are completely deaf, either unilateral or bilateral. In bilateral deaf subjects suffering from tinnitus, literature shows that cochlear implants (Cl) may cause tinnitus suppression; the experienced tinnitus level is reduced in $65 \%$ to $93 \%$ of the cases[10]. 
The criteria for $\mathrm{Cl}$ implantation widen over time. For example, audiological criteria for cochlear implantation have been adapted from bilateral total deafness (> $110 \mathrm{~dB} \mathrm{HL})$ in the early 1980 s, to severe hearing loss (> $70 \mathrm{~dB} \mathrm{HL}$ ) in the 1990s, and then to the current suprathreshold speech-based criteria (< $50 \%$ open-set sentence recognition with properly fitted hearing aids)[11]. Also, the age for cochlear implantation in children is reduced over the years. It is expected that it will only be a matter of time before cochlear implantation will be an acceptable treatment for unilateral deafness[12]. This may be one of the reasons why the effect of $\mathrm{Cl}$ on tinnitus in Single Sided Deafness (SSD) is nowadays under large scientific attention.

Cl as tinnitus therapy in SSD:

Table 1 summarizes all public available research documentation about the effect of $\mathrm{Cl}$ on tinnitus in SSD subjects. Eight of the nine published reports investigated the effect of "standard Cl" on tinnitus which means that the electrical stimulation depended on environmental sounds. In these cases, implanting and fitting a $\mathrm{Cl}$ in the deaf ear resulted in improved binaural hearing, especially in spatial conditions, as well as in improved directional hearing and localization $\left[13,14,16-19^{* *}, 22-24\right]$. One study reported the effect of an "experimental $\mathrm{Cl}$ " on tinnitus which means that the electrical stimulation was independent of environmental sounds. Here, no improvement of binaural hearing was expected.

Van de Heyning et al. reported the first study in which $\mathrm{Cl}$ was primary used as an option to treat unilateral tinnitus in SSD. In this study, 22 adults were included with unilateral severe, intractable tinnitus resulting from ipsilateral sensorineural deafness of various cochlear causes. Nine of them used a hearing aid in the non-implanted ear. Tinnitus loudness was measured with a visual analog scale (VAS). A score of 0 corresponds to "no tinnitus" while a score of 10 represents a "very loud, disturbing tinnitus". Furthermore, the Tinnitus Questionnaire (TQ) was used to evaluate the distress caused by the tinnitus. A higher TQscore represents more severe tinnitus complaints. VAS and TQ were administered 1 month prior to surgery and 1, 3, 6, 12, 18 and 24 months after the first fitting. After 24 months and based on VAS, this study reported complete tinnitus suppression in $14 \%$ of the subjects and tinnitus improvement in $82 \%$ of the subjects. Tinnitus loudness seemed to return partly after one hour of $\mathrm{Cl}$ deactivation. No worsening of tinnitus was reported. Tinnitus loudness seemed to stabilize after 3 to 6 months of $\mathrm{Cl}$ use and for these subjects the tinnitus loudness remained stable, at least up to 5 years after CI[13, 24]

Kleinjung et al. reported on a unilateral deaf man suffering from ipsilateral severe tinnitus by whom tinnitus was reduced 1 month after implantation and had disappeared completely 3 months postoperatively. TQ was used to measure the severity of tinnitus complaints, VAS was used to quantify tinnitus loudness and annoyance and Tinnitus Handicap Inventory (THI) to quantify the tinnitus related handicap[14].

Palau et al. investigated three subjects suffering from tinnitus who underwent $\mathrm{Cl}$ implantation. Subject 1 and 3 used a conventional $\mathrm{Cl}$ and subject 2 used a $\mathrm{Cl}$ with application of a noise habituator modulated via the $\mathrm{Cl}$ audio input (specially designed for the study). Based on THI and VAS, 6 months after implantation, tinnitus disappeared completely in subject 1 and tinnitus improved in subject 2 and 3 . The least tinnitus suppression was observed in the subject suffering more than 20 years of tinnitus, with application of noise habituator (subject 2)[15].

Buechner et al. published a study including five severe to profound unilateral deaf subjects suffering from ipsilateral tinnitus. Based on an average of four VAS scores, tinnitus suppression was observed in three subjects. Two of the subjects reported a nearly complete tinnitus reduction. The other two indicated that tinnitus could be reduced in certain situations[16]. Here, tinnitus possibly reoccurred due to psychological stress and a noisier work environment.

Arndt et al. reported another study including eleven SSD subjects, of which ten suffered from tinnitus. Based on VAS, at 6 months after $\mathrm{Cl}$-activation, five of the subjects reported a complete suppression of their tinnitus and three showed a tinnitus improvement. No tinnitus worsening was reported. When the $\mathrm{Cl}$ was deactivated, tinnitus reoccurred to initial tinnitus strength. However, of the two subjects in which tinnitus did not change after implantation with the $\mathrm{Cl}$ activated, one subjects reported an increase in tinnitus when the speech processor was deactivated[17].

Jacob et al. published the effects of $\mathrm{Cl}$ on the quality of hearing in unilateral deafness. Eleven of the thirteen included subjects suffered from tinnitus. They observed an improvement in the quality of hearing due to $\mathrm{Cl}$ implantation. As an additional effect they reported that nine subject declared that tinnitus was improved. No tinnitus worsening was reported. Quantification was not available[18*].

Ramos et al. performed a study including ten SSD subjects suffering from severe-to-profound suddenonset hearing loss and tinnitus in the affected ear, who all received a $\mathrm{Cl}$. Similar T-levels and C-levels of the electrode responsible for the tinnitus pitch and the four collateral electrodes were used. THI as well as VAS was used to quantify the tinnitus handicap and loudness respectively, at 1 and 3 months postoperatively. Two patients reported complete suppression of their tinnitus, while seven reported less tinnitus handicap and loudness. Again, no tinnitus worsening was observed. Interestingly, the improvement in tinnitus perception remained when the $\mathrm{Cl}$ was deactivated[19**].

Kleine Punte et al. published a study including 26 SSD-subjects undergoing Cl-implantation, all with unilateral severe-to-profound sensorineural hearing loss and suffering from severe tinnitus. 22 of them were already reported by Van de Heyning at al[13]. Based on VAS, 24 months after implantation, four of the subjects reported complete tinnitus suppression. The others reported tinnitus improvement. In 24 cases tinnitus reoccurred at the original loudness after $\mathrm{Cl}$ deactivation. Two subjects did not experience any tinnitus within one day after $\mathrm{Cl}$ deactivation. Four subjects (S23-S26), which were not described by Van de Heyning et al. [13] were followed up to 12 months post-operative. Effects on tinnitus loudness were comparable to the 22 subjects described earlier. Tinnitus Loudness seemed to be stabilized after 3 to 6 months post-operatively. Furthermore, no differences were observed between patients suffering from pure-tone tinnitus, narrow band noise tinnitus or polyphonic tinnitus [20**]. 
Table 1: Summary of published research on Cl as a treatment for tinnitus in SSD. Cl: cochlear implant, THI: Tinnitus Handicap Inventory, TQ: Tinnitus Questionnaire and VAS: Visual Analog Scale.

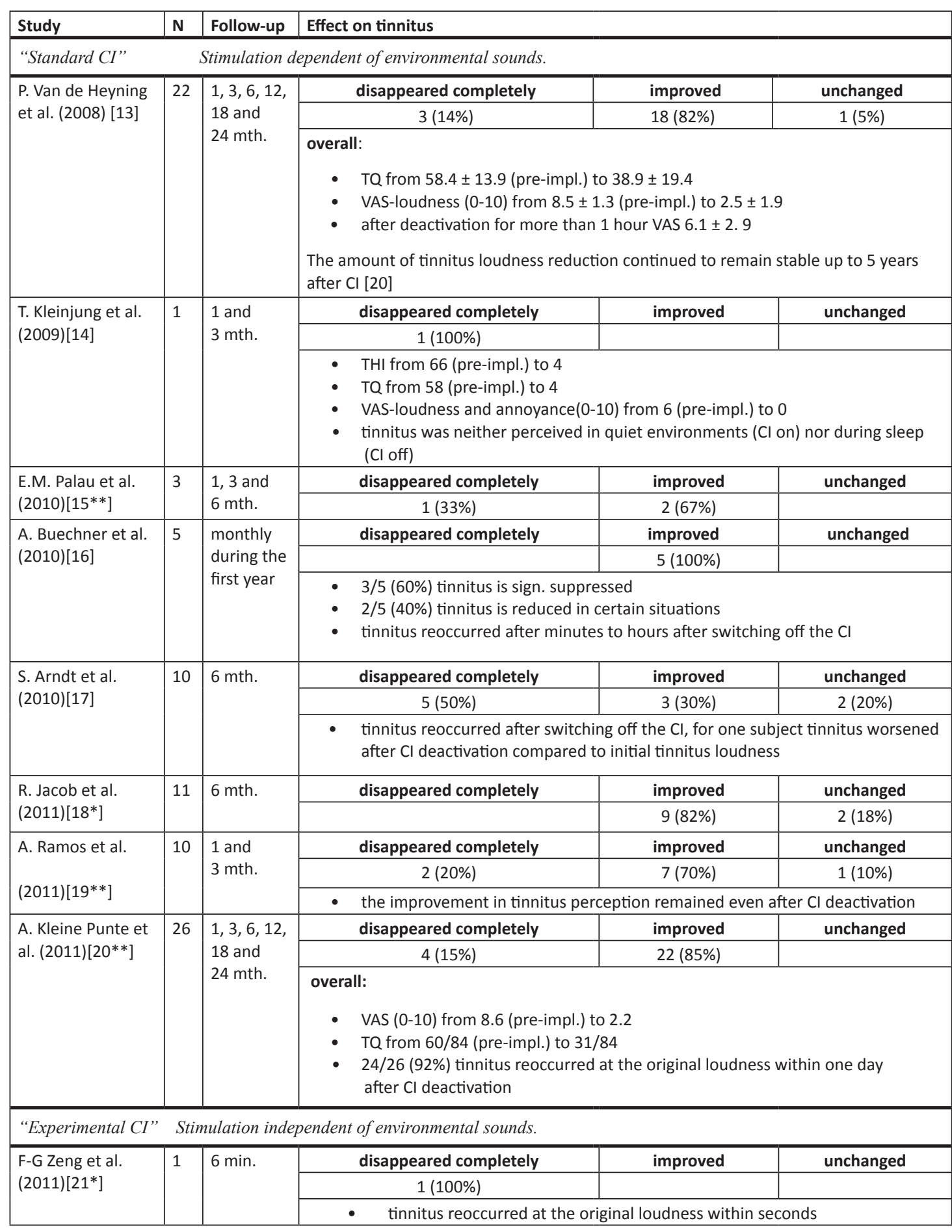

Zeng et al. reported a study with a study design different from the others in which they used experimenta $\mathrm{Cl}$ settings as an attempt to suppress tinnitus in one SSD subject. Here, the quality of hearing was not improved as they used an electrical stimulation pattern independent of environmental sounds. The reason why they did this is because there was no tinnitus reduction observed with the standard clinical $\mathrm{Cl}$ setting. Based on VAS, tinnitus was completely suppressed for six minutes using a certain stimulus. Tinnitus reoccurred at original loudness within seconds after stopping intracochlear electrical stimulation[21*].

\section{Discussion:}

Based on the publicly available reports investigating the effect of $\mathrm{Cl}$ on tinnitus in SSD, cochlear implantation seems to be an appropriate treatment to suppress tinnitus, which is considered to arise from cochlear deafferentiation. Tinnitus does not restore during the electrical stimulation presented by the Cl. This result is comparable with studies investigating tinnitus suppression after cochlear implantation in bilateral deafness[25-27]. Sporadically a deterioration of tinnitus can be observed in bilateral deaf patients after Cl implantation [26, 28]. This does not seem to be the case in SSD patients which suffer from pre-operative tinnitus. Furthermore, the tinnitus level seems to stabilize 3 to 6 months after the first fitting. There is no indication for any relation between the tinnitus duration before implantation and the level of tinnitus suppression. Finally, no differences were observed between pure-tone tinnitus, narrow band noise tinnitus or polyphonic tinnitus.

As an attempt to increase power, data from several studies are pooled. To do this in an appropriate way only data from identical study designs and outcome measures are included. However, as already explained, Ramos et al.[19**] used a different intervention in which five electrodes had the same T-levels and C-levels. Paired sample t-test (two-tailed) were accomplished when normally distributed differences between pre- and post-operative variables could be found (Kolmogorov-Smirnov: $\alpha=0.05$ ). Otherwise, Wilcoxon Signed Rank Test was used. Table 2 shows quantification for testing differences in mean. Data is considered as a multi-center study.

Table 2: Testing differences in mean for pooled data. Tinnitus loudness measured by means of VAS $(0-10)$. Subjects are excluded if data was missing so caution for selection bias must be emphasized. Quantification is obtained using SPSS.

\begin{tabular}{|c|c|c|c|c|c|}
\hline & pooled data & $\begin{array}{c}\text { testing } \\
\text { normally } \\
\text { distribution }\end{array}$ & $\begin{array}{c}\text { pre-op. } \\
\text { mean } \pm \text { std }\end{array}$ & $\begin{array}{c}\text { post-op. } \\
\text { mean } \pm \text { std }\end{array}$ & $\begin{array}{c}\text { testing } \\
\text { differences in } \\
\text { mean }\end{array}$ \\
\hline $\begin{array}{c}\text { pre-op. vs. 1 mth. } \\
\text { post-op. }\end{array}$ & $\begin{array}{c}\mathrm{N}=23 \\
{[13,14]}\end{array}$ & $\mathrm{p}=0.2$ & $8.35 \pm 1.36$ & $3.20 \pm 2.38$ & $\mathrm{p}=0.000$ \\
\hline $\begin{array}{c}\text { pre-op. vs. 3 mth. } \\
\text { post-op. }\end{array}$ & $\begin{array}{c}\mathrm{N}=33 \\
{\left[13,14,19^{* *}\right]}\end{array}$ & $\mathrm{p}=0.027$ & $8.21 \pm 1.57$ & $2.29 \pm 1.87$ & $\mathrm{p}=0.000$ \\
\hline $\begin{array}{c}\text { pre-op. vs. 6 mth. } \\
\text { post-op. }\end{array}$ & $\begin{array}{c}\mathrm{N}=31 \text {. } \\
{[13,17]}\end{array}$ & $\mathrm{p}=0.06$ & $7.84 \pm 0.36$ & $2.15 \pm 0.31$ & $\mathrm{p}=0.000$ \\
\hline $\begin{array}{c}\text { pre-op. vs. 12 } \\
\text { mth. post-op. }\end{array}$ & $\begin{array}{c}\mathrm{N}=19 \mathbf{} \\
{\left[13,20^{* *}\right]}\end{array}$ & $\mathrm{p}=0.2$ & $8.68 \pm 1.04$ & $2.52 \pm 1.72$ & $\mathrm{p}=0.000$ \\
\hline
\end{tabular}


After testing pooled data, statistical evidence can be found for differences in mean as regards tinnitus loudness (VAS 0-10) between pre-op and 1, 3, 6 as well as 24 months post-op ( $p=0.000$ for each test).

However, it must be considered that these studies have some limitations like the lack of a control group, the absence of any blinding and the small numbers of included subjects. Furthermore, caution is required because of possible publication bias.

Even though $\mathrm{Cl}$ appears to be an appropriate treatment for tinnitus in SSD, the responsible underlying mechanism is still unknown. Implantation increases afferent information in the auditory nerve, which may reduce tinnitus as it reverses the possibly responsible neural changes. However, the improved hearing after cochlear implantation also results in less direct awareness of the tinnitus, which is possibly a confounding effect. Zeng et al. reported in a case-report that tinnitus disappeared after intracochlear electrical stimulation, independent of environmental sounds, generated by the $\mathrm{Cl}$ in a SSD subject[21*]. This seems to give evidence for the increased afferent information in the auditory nerve as the mechanism responsible for the observed suppression. Although, it must be emphasized that it is a case-report and the stimulus duration was only six minutes. Therefore, it cannot be excluded that this effect is due to placebo-effect for which tinnitus patients are very sensitive. We plan a study including SSD subjects suffering from tinnitus which will receive long-term intracochlear electrical stimulation. To get insight in which mechanism is responsible for the observed tinnitus suppression after cochlear implantation, participants will receive electrical stimulation independent of environmental sounds. Possibly tinnitus can be suppressed further due to stimulus optimization.

\section{Conclusion:}

All the recent studies assessed in the current review observe tinnitus suppression in the majority of the included SSD subjects undergoing cochlear implantation. None of the studies reported tinnitus worsening after $\mathrm{Cl}$ implantation. Furthermore, no restoration of the tinnitus to the electrical stimulation presented by the $\mathrm{Cl}$ can be observed.

Although the underlying mechanism responsible for the observed tinnitus suppression is not yet clear, cochlear implantation should be considered as a viable treatment option for tinnitus arising from single sided deafness. However, appropriate patient selection is essential as it is expected that it is a requirement that tinnitus arises from cochlear deafferentiation.

\section{Key bullet points:}

- The majority of the SSD subjects suffering from tinnitus observed tinnitus improvement after cochlear implantation.

- None of the studies reported tinnitus worsening after cochlear implantation.

- No restoration of the tinnitus to the electrical stimulation presented by the $\mathrm{Cl}$ was observed.
References

Papers of particular interest have been highlighted as:

* Of special interest

** Of outstanding interest

[1] Hall DA, Láinez MJ, Newman CW, Sanchez TG, Egler M, Tennigkeit F, Koch M, Langguth B. Treatment options for subjective tinnitus: self report from a sample of general practitioners and ENT physicians within Europe and the USA. BMC Health Services Research 2011;11:302

[2] Tyler RS, Rubinstein J, Pan T, Chang S, Gogel SA, Gehringer A, Coelho C. Electrical stimulation of the cochlea to reduce tinnitus. Semin Hear 2008; 29: 326-332

[3] Eggermont JJ, Roberts LE. The neuroscience of tinnitus. TRENDS in Neuroscience 2004; Vol. 27 No. 11: 676-682

[4] Langers D, de Kleine E, van Dijk P. Tinnitus does not require macroscopic tonotopic map reorganization. Frontiers in Systems Neuroscience 2012; Vol. 6 (2): 1-15

[5] Westin VZ, Schulin M, Hesser H, Karlsson M, Noe RZ, Olofsson U, Stalby M, Wisung G, Andersson G. Acceptance and Commitment Therapy versus Tinnitus Retraining Therapy in the treatment of tinnitus: A randomised controlled trial. Behaviour Research and Therapy 2011; $x x x: 1-11$

[6] Quaranta N, Fernandez-Vega S, D'Elia C, Filipo R, Quaranta A. The effect of unilateral multichannel cochlear implant on bilateral perceived tinnitus. Acta Oto-Laryngologica 2008; 128: 159-163

[7] Parazzini M, Bo LD, Jastreboff M, Tognola G, Ravazzani P. Open ear hearing aids in tinnitus therapy: an efficacy comparison with sound generators. International Journal of Audiology 2011; 50:548-553

[8] Henry JA, Loovis C, Montero M, Kaelin C, Anselmi KA, Coombs R, Hensley J, James KE. Randomized clinical trial: group counseling based on tinnitus retraining therapy. J Rehabil Res Dev 2007; 44(1):21-32

[9] Jastreboff PJ. Phantom auditory perception (tinnitus): mechanism of generation and perception. Neurosci Res 1990; 8: 221-254

[10] Aschendorff A, Pabst G, Klenzner T, Laszig R. Tinnitus in cochlear implant users: the Freiburg experience. International Tinnitus Journal 1998; Vol. 4 No. 2: 162-164

[11] Zeng FG. Trends in cochlear implants. Trends Amplif 2004; 8(1): 1-34

[12] Sampaio A, Araújo M, Oliveira C. New criteria of indication and selection of patients to cochlear implant. International Journal of Otolaryngology 2011 
[13] Van de Heyning, Vermeire K, Diebl M, Nopp P, Anderson I, De Ridder D. Incapacitating unilateral tinnitus in single-sided deafness treated by cochlear implantation. Annals of otology, rhinology \& Laryngology 2008; 117(9): 645-652

[14] Kleinjung T, Steffens T, Strutz J, Langguth B. Curing tinnitus with a cochlear Implant in a patien with unilateral sudden deafness: a case report. Cases Journal 2009; 2: 7462

[15] Palau EM, Gil J, Vidal C, González J, Cabrera O, Macías Á. Tinnitus and cochlear implantation Preliminary experience. Acta Otorrinolaringol Esp. 2010; 61(6):405-411

[16] Buechner A, Brendel M, Lesinski-Schiedat A, Wenzel G, Frohne-Buechner C, Jaeger B, Lenarz Cochlear implantation in unilateral deaf subjects associated with ipsilateral tinnitus. Otology \& Neurotology 2010; 31: 1381-1385

[17] Arndt S, Aschendorff A, Laszig R, Beck R, Schild C, Kroeger S, Ihorst G, Wesarg T. Comparison of pseudobinaural hearing to real binaural hearing rehabilitation after cochlear implantation in patients with unilateral deafness and tinnitus. Otology \& Neurotology 2010; 32:39-47

*[18] Jacob R, Stelzig Y, Nopp P, Schleich P. Audiologische Ergebnisse mit Cochleaimplantat bei einseitiger Taubheit. HNO 2011; 59: 453-460

Nine of the eleven included subjects in this study reported tinnitus suppression.

However, not any quantification was available.

**[19] Ramos Á, Polo R, Masgoret E, Artiles O, Lisner I, Zaballos M, Moreno C, Osoria Á. Cochlear implant in patients with sudden unilateral sensorineural hearing loss and associated tinnitus. Acta otorrinolaringol Esp. 2012; 63(1): 15-20

This study reported $\mathrm{Cl}$ as a valid and effective tinnitus therapy when other treatments have failed.

**[20] Kleine Punte A, Vermeire K, Hofkens A, De Bodt M, De Ridder D, Van de Heyning P. Cochlear implantation as a durable tinnitus treatment in single-sided deafness. Cochlear Implant International 2011; Vol. 12 No. S1

In this study, no differences were observed between patients with pure-tone tinnitus, narrow band noise tinnitus, or polyphonic tinnitus.

*[21] Zeng FG, Tang Q, Dimitrijevic A, Starr A, Larky J, Blevins NH. Tinnitus suppression by low-rat electric stimulation and its electrophysiological mechanisms. Hearing Research 2011:277: 61

This study is a case report. No tinnitus reduction was found using the standard clinical $\mathrm{Cl}$ setting. Complete tinnitus suppression was reported after applying electrical stimulation independent of environmental sounds.

[22] Vermeire k, Van de Heyning P. Binaural hearing after cochlear implantation in subjects with unilateral sensorineural deafness and tinnitus. Audiol Neurotol 2009; 14: 163-171
Stelzig Y, Jacob R, Mueller J. Preliminary speech recognition results after cochlear implantatio in patients with unilateral hearing loss: a case series. Journal of Medical Case Reports 2011; 5 343

[24] Van de Heyning P, Kleine Punte A, De Bodt M, De Ridder D. Long-term tinnitus relief after cochlear implantation in single-sided deafness. Abstract. Tinnitus Research Initiative 2011 Iowa USA

[25] Ito J, Sakakihara J. Suppression of tinnitus by cochlear implantation. American Journal of Otolaryngology 1994; Vol. 15 No. 2:145-148

[26] Nardo W, Cantore I, Cianfrone F, Melillo P, Scorpecci A, Paludetti G. Tinnitus Modifications after cochlear implantation. Eur Arch Otorhinolaryngol 2007; 264: 1145-1149

[27] Amoodi H, Mick p, Shipp D, Friesen L, Nedzelski J, Chen J, Lin V. The effects of unilatera cochlear implantation on the tinnitus handicap inventory and the influence on quality of life. Laryngoscope 2011; 121: 1536-40

[28] Bovo R, Ciorba A, Martini A. Tinnitus and cochlear implants. Auris Nasus Larynx 2010 doi:10.1016/j.anl.2010.05.003 


\section{Chapter}

The occurrence of tinnitus after $\mathrm{Cl}$ surgery in patients with severe hearing loss: A retrospective study.

$$
\begin{aligned}
& \text { R.A.G.J. Artsa, } \\
& \text { T. Netz } \\
& \text { A.M.L. Janssena }{ }^{\mathrm{b}} \text {, } \\
& \text { E.L.J. George } \\
& \text { R.J. Stokroos } \\
& \text { a }
\end{aligned}
$$

aDepartment of Otorhinolaryngology, Head and Neck Surgery, Maastricht University Medical Center; MHeNS School for Mental Health and Neuroscience, Maastricht, the Netherlands

bDepartment of Methodology and Statistics,

Maastricht University; CAPHRI School for Public Health and Primary Care, Maastricht, the Netherlands

Int J Audiol. 2015 Sep 24:1-8. [Epub ahead of print] D Ol:10.3109/14992027.2015.1079930

Key words: Tinnitus, Cochlear Implant, Cl-surgery, retrospective

mens niet slechts a1s midde1, tegelijkertijd ook a1s doe 1 Kant (1724-1804) 
Abstract:

Objective: An often reported side-effect of a cochlear implant (CI) is suppression of tinnitus. However, induction of tinnitus is also a known complication of $\mathrm{Cl}$-surgery. To date, it remains unclear why some $\mathrm{Cl}$ recipients experience larger tinnitus complaints due to $\mathrm{Cl}$-surgery while most others do not. The goal of this study was to investigate the effect of deterioration of residual hearing due to $\mathrm{Cl}$ surgery on tinnitus.

Design: In this retrospective database study, pre- and postoperative audiometric hearing thresholds were retrieved from patients' clinical files. Furthermore, tinnitus questionnaires were sent to all patients who fitted inclusion criteria.

Study Sample: Of the $512 \mathrm{Cl}$-patients who were implanted within the last ten years, 197 patients fitted the inclusion criteria and returned the questionnaires.

Results: We identified 39 cases (19.8\%) who suffered from a newly developed (25) or deteriorated (14) tinnitus following $\mathrm{Cl}$ surgery. No statistically significant association between perioperative deterioration of hearing thresholds measured by pure-tone audiometry and the development or deterioration of tinnitus was found.

Conclusions: There is a small risk for deteriorated residual hearing after cochlear implantation. However for patients with preoperative residual hearing, there appears to be no increased risk of developing chronic tinnitus after hearing deterioration due to cochlear implantation.

\section{List of abbreviations:}

\section{C.I. confidence interval}

$\mathrm{Cl}$ cochlear implant

$\mathrm{dB}$ decibels

$\mathrm{dBHL}$ decibels Hearing Leve

ExpB exponent of the regression coefficient

$\mathrm{HD}$ hearing deterioration

$\mathrm{kHz} \quad$ kilohertz

OR odds ratio

Periop. perioperative

Postop. postoperative

Preop. preoperative

PTA pure-tone audiometry

SD standard deviation

TQ Tinnitus Questionnaire

VAS Visual Analogue Scale

\section{Introduction:}

Tinnitus aurium, which means "ringing of the ears", can be divided into objective and subjective tinnitus. Objective tinnitus is defined as a sound that originates from an acoustic source within the patient and is audible for an examiner, while subjective tinnitus has no acoustic source (Erlandsson \& Dauman, 2013), accounting for $95 \%$ of all tinnitus cases. Subjective tinnitus has an estimated prevalence of $8-15 \%$ in the general adult population (Langguth et al., 2013; Nondahl et al., 2002) and a relatively higher prevalence is observed among the elder compared to the younger population (Lockwood et al., 2002; Engdahl et al., 2012). The variability in prevalence is largely explained by the slight differences in definitions of tinnitus used. Hearing loss due to aging has been shown to be a major risk factor for its development (Knipper et al., 2013). Considering the demographic changes in western societies and the increasing noise exposure among the younger population, the prevalence of tinnitus is expected to further increase during the following decades (Gilles et al., 2013).

Tinnitus significantly reduces the quality of life, may cause stress, depression, anxiety and may be the cause of suicidal tendencies for some patients (Turner et al., 2007; Schaaf et al., 2009). A recent study in the Netherlands calculated mean annual health care costs of $€ 1544$ per tinnitus patient as a consequence of frequent consultation of different health professionals (Maes et al., 2013).

There is no unambiguous evidence about the cause of tinnitus, but numerous studies have led to severa theories about its etiology. Section of the auditory nerve does not cure the patient from tinnitus, suggesting an involvement of the central nervous system (Jackson, 1985). Different homeostatic processes along the auditory pathway to compensate for complete hearing loss or partially reduced hearing are plausible causes of tinnitus. These homeostatic processes may be tonotopic reorganization (Eggermont \& Roberts, 2012), neural response gain: an up-regulation of the neural responsiveness through the brainstem (Schaette \& McAlpine, 2011) and an imbalance in inhibitory and excitatory neurotransmitters (Bauer et al., 2013).

Within the past decade, cochlear implantation is commonly applied as a treatment in patients with severe bilateral sensorineural hearing loss. An often reported side-effect is that the Cochlear Implant (C) seems to suppress tinnitus in bilateral deaf as well as in unilateral deaf patients (Van de Heyning et al, 2008; Amoodi et al., 2011; Arts et al., 2012; Kompis et al., 2012; Olze et al., 2012). Auditory stimulation by a Cl appears to suppress tinnitus ipsilateral and contralateral to the implanted side (Demajumdar et al., 1999). Moreover, a positive effect of the $\mathrm{Cl}$ surgery itself on tinnitus is sporadically reported (Arts et al. 2015; Klootstra et al., 2015). However, induction of tinnitus is also a known complication of $\mathrm{Cl}$ surgery, although the incidence varies considerably among the studies; ranging from $0 \%$ to $23.5 \%$ postoperatively (Webb et al., 1991; Greimel et al., 2003; Quaranta et al., 2004; Di Nardo et al., 2007; Postelmans et al . 2007; Akdogan et al., 2009; Pan et al., 2009; Hou et al., 2010; Kloostra et al., 2015; Todt et al., 2015).

To date, it remains unclear why some $\mathrm{Cl}$-recipients experience tinnitus complaints due to $\mathrm{Cl}$ surgery while most others do not. A possible explanation is that deterioration of residual hearing as a result of traumatic insertion of the electrode array may trigger the experience of tinnitus postoperatively (Todt et al., 2014; 2015). The goal of this study was to investigate the effect of deterioration of residual hearing due to $C$ surgery on tinnitus development. This is increasingly important as the criteria for $\mathrm{Cl}$ widen over time and cochlear implantation is more often considered in patients with significant residual hearing. Furthermore, knowledge concerning the mechanism responsible for changes in tinnitus experience as a result of $\mathrm{Cl}$ surgery has relevance for patient counselling and informed consent. Finally, insight of the underlying 
mechanism of tinnitus as a complication of $\mathrm{Cl}$ surgery contributes to the general knowledge about the origin of tinnitus.

\section{Materials and Methods:}

\section{Subjects}

The Cl-team of South-East Netherlands, based at the Maastricht University Medical Center, has been fitting patients with cochlear implants since 2001. A total of $512 \mathrm{Cl}$-patients were implanted within the last ten years (from 2003 to 2013) and were retrospectively available as research population. Cl-patients who were implanted more than ten years ago and patients older than 85 were excluded because of expected difficulties remembering preoperative aspects of their tinnitus. Patients younger than 18 years were excluded from participation to conform with ethical regulations. Furthermore, patients experiencing pulsatile tinnitus were excluded from this study as it could be an indication for objective tinnitus; patients with diagnosed objective tinnitus were also excluded.

In this retrospective database study, audiometric hearing thresholds, measured by pure-tone audiometry (PTA) were retrieved from patients' clinical files, both preoperatively (less than a month before Cl surgery) and 6 months postoperatively.

The indication for unilateral $\mathrm{Cl}$ in the Netherlands is bilateral severe hearing loss (OPCl, 2005). To be able to investigate possible further loss of residual hearing due to surgery, patients without any preoperative residual hearing were excluded (thresholds larger than $100 \mathrm{~dB} \mathrm{HL}$ at 0.25 and $8 \mathrm{KHz}$, and larger than 120 $\mathrm{dB} \mathrm{HL}$ at $0.5,1,2$ and $4 \mathrm{kHz}$ ). Finally, patients with bilateral Cl's were excluded, as the non-implanted contralateral ear was used as control.

Moreover, this retrospective database study was compared to an age-matched reference population from the Blue Mountains Hearing Study (Gopinath et al., 2010). Here, 811 participants without tinnitus at baseline were followed for five years.

\section{Methods}

Questionnaires concerning tinnitus experiences (see appendix; accessible via http://informahealthcare. $\mathrm{com} / \mathrm{loi} / \mathrm{ija}$ ) were sent to all patients who fitted inclusion criteria. For all patients who returned their questionnaires, pre- and postoperative PTA were studied to investigate pre- and postoperative hearing loss, comparing the patients who developed tinnitus following $\mathrm{Cl}$ surgery with those who do not. Gender and $\mathrm{Cl}$ brand were included in the analysis as covariates. Descriptive statistics were performed on implanted electrode array since length and thickness of the electrode array may be expected to affect residual hearing.

\section{Outcome measurements}

PTA was performed under earphones (TDH 39, Telephonics; New York; NY) at frequencies 0.25, 0.5, 1, 2, 4 and $8 \mathrm{kHz}$, as part of our standard clinical routine.

In order to avoid false positive thresholds due to vibrotactile sensations strict criteria were applied to the obtained thresholds. Cutoff points for vibrotactile sensations were earlier found to be around 100dB HL for 0.25 and $8 \mathrm{KHz}$ and around 120dB HL for 0.5, 1, 2 and 4KHz (Boothroyd \& Cawkwell, 1970). Therefore, taking the accuracy of the PTA into account, we additionally excluded patients with preoperative hearing thresholds of $90 \mathrm{~dB} \mathrm{HL}$ or more for 0.25 and $8 \mathrm{KHz}$ and $110 \mathrm{~dB} \mathrm{HL}$ or more for $0.5,1,2$ and $4 \mathrm{KHz}$ on the $\mathrm{Cl}$ - side. For the analyses, no responses were indicated as the limit of the audiometer at this specific frequency (see cutoff points for vibrotactile sensations) plus $5 \mathrm{~dB}$.

Deterioration of hearing loss as a result of surgery was defined according to our clinical criteria: postoperative deterioration of $15 \mathrm{~dB}$ or more in one frequency and/or $10 \mathrm{~dB}$ or more in three frequencies, compared to preoperative PTA. As a control for confounding through external factors that might have altered the hearing as well, the contralateral ears were examined, handling the same criteria for deterioration of hearing thresholds.

A questionnaire concerning pre- and postoperative tinnitus loudness and postoperative tinnitus pitch as well as questions to identify objective tinnitus was used (see appendix; accessible via http:// informahealthcare.com/loi/ija). The questionnaire explicitly focused on postoperative tinnitus experiences in situations when the Cl-processor was not used, to avoid bias from intracochlear electrical stimulation. Patients with preexistent tinnitus were asked to report changes in tinnitus loudness; all patients with postoperative tinnitus were asked to additionally fill out the validated Dutch version of the Tinnitus Questionnaire (TQ) (Meeus et al., 2007; Zeman et al., 2012). The TQ relates to the postoperative tinnitus and consists of 52 items about the severity of tinnitus that can be answered, "true", "partly true" and "not true". Patients can score a maximum of 84 points and are divided into four groups of tinnitus severity: mild 0-30, moderate 31-46, severe 47-59 and very severe tinnitus 60-84 (Adamchic et al., 2012).

\section{Ethics}

This study was conducted in accordance with national legislation, the medical-ethical standards of the institutional review board and the Declaration of Helsinki. Patients included in the study population declared no objection to the use of clinically obtained data for medical research.

\section{Statistics}

Descriptive statistics include mean and standard deviation (SD) or percentage and quantitative data are presented as absolute frequencies. The Fisher exact test was performed to assess the association between perioperative hearing deterioration and TQ-score. The McNemar's test for correlated proportions was used to test whether hearing deterioration was associated to $\mathrm{Cl}$-surgery. A series of univariable logistic regression analyses were performed with perioperative tinnitus as dependent variable and perioperative hearing deterioration, gender and $\mathrm{Cl}$ brand as independent variables. A multivariable logistic regression model was constructed to examine the effect of perioperative deterioration of hearing thresholds on perioperative tinnitus after adjustment for gender and $\mathrm{Cl}$ brand. Furthermore, in the multivariable model the potential interaction between perioperative hearing deterioration and gender was examined. The odds ratios (OR) are presented along with the $95 \%$ confidence intervals ( $95 \%$ C.I.) and the corresponding $p$-values. Binomial tests were used to test for differences in tinnitus incidence in an age-matched population. $P$-values $<0.05$ were considered statistically significant. Data analysis was performed using IBM SPSS Statistics version 20 (IBM Corp., Armonk, NY).

\section{Results:}

Of the 512 patients who underwent $\mathrm{Cl}$ surgery within the last ten years, 265 did not meet the inclusion criteria. From the remaining 247 patients to whom a questionnaire was sent, 42 did not respond and 8 questionnaires were filled in invalidly (response rate: $80 \%$ ). Of the 197 patients who responded validly, $106(53.8 \%)$ had no postoperative tinnitus at all, $25(12.7 \%)$ had developed tinnitus after the implantation 
and $66(33.5 \%)$ patients suffered from preoperative as well as postoperative tinnitus. The patients who experienced tinnitus preoperatively as well as postoperatively were subdivided into deterioration (14), no change (31) and improvement (21) of the postoperative tinnitus complaints compared to preoperative complaints. In total we identified 39 cases who suffered from a newly developed (25) or deteriorated tinnitus (14) following $\mathrm{Cl}$ surgery (Figure 1), and this group was defined as perioperative tinnitus. The 106 patients who do not experience tinnitus postoperatively were not further split into groups as it was not relevant for the current research question.

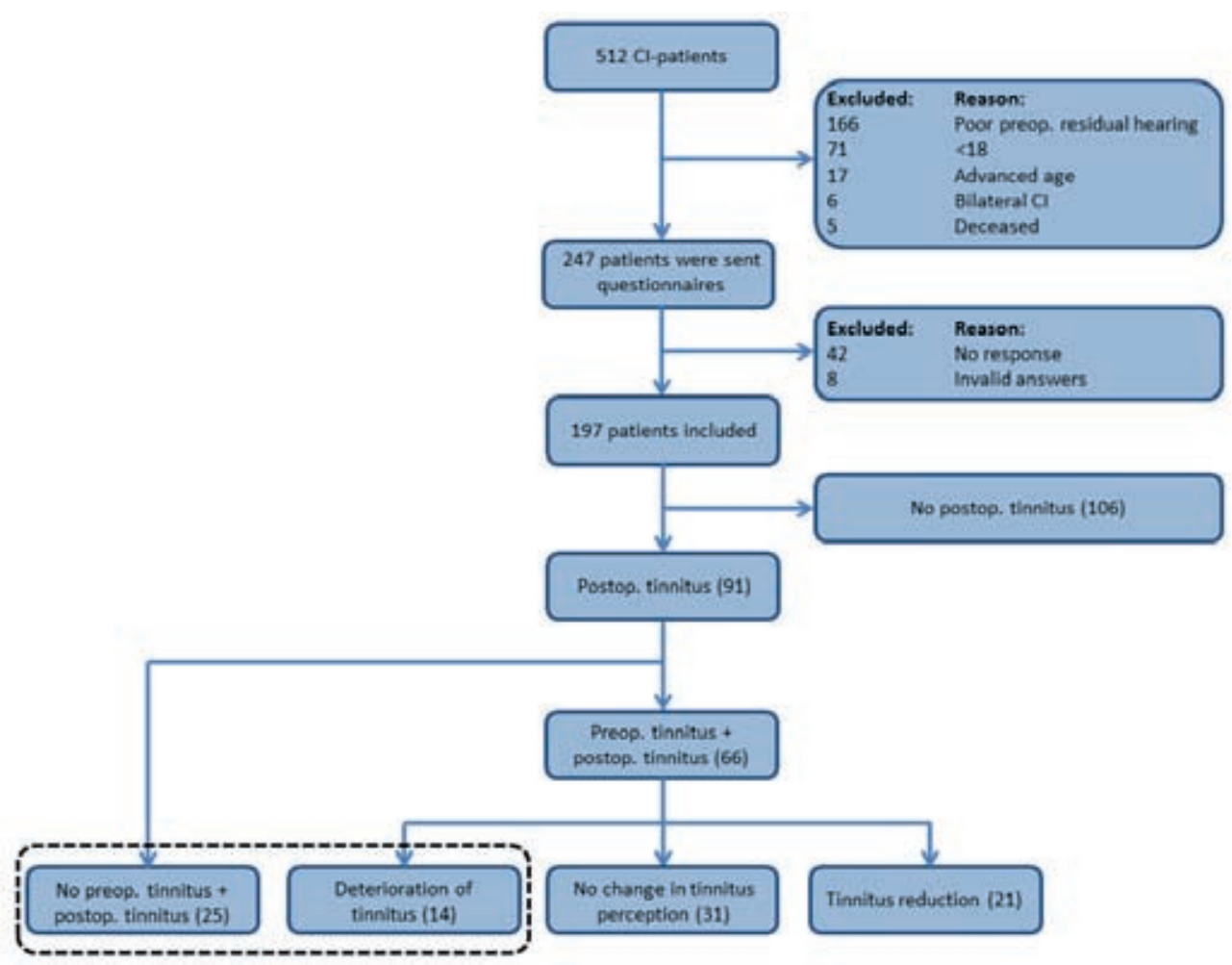

Figure 1: Flowchart. The perioperative tinnitus group is marked with a dashed line. CI: Cochlear Implant; Preop.: preoperative; Postop.: postoperative

Of the 39 patients who developed perioperative tinnitus, in 27 patients the tinnitus developed in the implanted ear while in 11 patients the tinnitus deteriorated in the implanted ear. In one patient the tinnitus, perceived in the non-implanted ear, deteriorated.
Table 1: Demographics

\begin{tabular}{|c|c|c|c|c|}
\hline \multirow{2}{*}{\multicolumn{2}{|c|}{ Variable }} & \multicolumn{2}{|c|}{ Perioperative tinnitus } & \multirow{2}{*}{$\begin{array}{c}\text { Total } \\
(\mathrm{N}=197)\end{array}$} \\
\hline & & No $(N=158)$ & Yes $(\mathrm{N}=39)$ & \\
\hline \multicolumn{2}{|l|}{ Gender } & & & \\
\hline \multicolumn{2}{|l|}{ Male } & 67 & $22(24.7 \%)$ & $89(100.0 \%)$ \\
\hline \multicolumn{2}{|l|}{ Female } & 91 & $17(15.7 \%)$ & $108(100.0 \%)$ \\
\hline \multicolumn{2}{|c|}{ Mean age in years (SD) } & $62.5(14.6)$ & $58.0(13.6)$ & $61.6(14.5)$ \\
\hline \multicolumn{2}{|c|}{ Mean Cl-use in years (SD) } & $4.6(2.8)$ & $5.1(2.9)$ & $4.7(2.8)$ \\
\hline \multicolumn{2}{|l|}{ Implanted ear } & & & \\
\hline \multicolumn{2}{|l|}{ Right ear } & 82 & $26(24 . \%)$ & $108(100.0 \%)$ \\
\hline \multicolumn{2}{|l|}{ Left ear } & 76 & $13(14.6 \%)$ & $89(100.0 \%)$ \\
\hline \multicolumn{2}{|c|}{ Hearing preservation } & & & \\
\hline \multicolumn{2}{|c|}{ No perioperative hearing deterioration } & 65 & $16(19.8 \%)$ & $81(100.0 \%)$ \\
\hline \multicolumn{2}{|c|}{ Perioperative hearing deterioration } & 93 & $23(19.8 \%)$ & $116(100.0 \%)$ \\
\hline \multicolumn{2}{|l|}{ Cl's brand } & & & \\
\hline \multirow{3}{*}{$\begin{array}{l}\text { Advanced Bionics } \\
\text { Cochlear } \\
\text { MED-EL }\end{array}$} & (Advanced Bionics LLC, Sylmar, CA) & 32 & $10(23.8 \%)$ & $42(100.0 \%)$ \\
\hline & (Cochlear Ltd. Sydney, Australia) & 108 & $21(16.3 \%)$ & $129(100.0 \%)$ \\
\hline & (MED-EL Corp., Innsbruck, Austria) & 18 & $8(30.8 \%)$ & $26(100.0 \%)$ \\
\hline \multicolumn{2}{|l|}{ Electrode array } & & & \\
\hline Hi Focus Helix & (Advanced Bionics LLC, Sylmar, CA) & 24 & $8(25.0 \%)$ & $32(100.0 \%)$ \\
\hline Hi Focus $1 J$ & (Advanced Bionics LLC, Sylmar, CA) & 11 & $3(21.4 \%)$ & $14(100.0 \%)$ \\
\hline Contour Advance & (Cochlear Ltd., Sydney, Australia) & 71 & $16(18.4 \%)$ & $87(100.0 \%)$ \\
\hline Slim Straight & (Cochlear Ltd., Sydney, Australia) & 22 & $2(8.3 \%)$ & $24(100.0 \%)$ \\
\hline Contour & (Cochlear Ltd., Sydney, Australia) & 9 & $1(10.0 \%)$ & $10(100.0 \%)$ \\
\hline Flex 28 & (MED-EL Corp., Innsbruck, Austria) & 8 & $2(20.0 \%)$ & $10(100.0 \%)$ \\
\hline Unknown & (MED-EL Corp., Innsbruck, Austria) & 2 & $1(33.3 \%)$ & $3(100.0 \%)$ \\
\hline Other & & 11 & $6(35.3 \%)$ & 17 (100.0\%) \\
\hline
\end{tabular}

Cl: cochlear implant, SD: standard deviation

In three included Cl-recipients, the implanted electrode array is unknown (Table 1). However, of these unknown electrode arrays the Cl brand is MED-EL (MED-ELCorp., Innsbruck, Austria). The most frequently implanted electrode array was the "Contour Advance" from Cochlear (Cochlear Ltd., Sydney, Australia) of which 18.4\% developed perioperative tinnitus. Advanced Bionics' (Advanced Bionics LLC, Sylmar, CA) and MED-EL's (MED-ELCorp., Innsbruck, Austria) most frequently implanted electrode array were significantly less often implanted and do not seem to be different in perioperative tinnitus development, compared to the "Contour Advance" electrode array.

Table 2 shows that the proportion of hearing deterioration for implanted ears (58.9\%) significantly differs from the proportion of hearing deterioration for contralateral ears $(9.1 \%, p<0.001)$. Figure 2 shows the averaged pre- and postoperative audiometric hearing thresholds of both the "perioperative tinnitus" group and the "no perioperative tinnitus" group. 
Table 2: Association between Cl-surgery and perioperative hearing deterioration.

\begin{tabular}{|c|c|c|c|c|}
\hline & No HD non-implanted ear & HD non-implanted ear & Total & \multirow{4}{*}{$\begin{array}{l}\text { McNemar's test: } \\
\quad P<0.001\end{array}$} \\
\hline No HD implanted ear & $74(37.6 \%)$ & $7(3.6 \%)$ & 81 (41.1\%) & \\
\hline HD implanted ear & 105 (53.3\%) & $11(5.6 \%)$ & 116 (58.9\%) & \\
\hline Total & $179(90.9 \%)$ & $18(9.1 \%)$ & $197(100.0 \%)$ & \\
\hline
\end{tabular}

$\mathrm{HD}$ : hearing deterioration

The results of the univariable and multivariable logistic regression analyses are shown in Table 3 . Unadjusted univariable logistic regression analysis shows no statistically significant association between perioperative deterioration of hearing thresholds and perioperative tinnitus. Furthermore, univariable analyses also show no significant associations between perioperative tinnitus and the covariates gender and Cl's brand. The implanted electrode array could not be included as a covariate due to the limited amount of cases with perioperative tinnitus.

No perioperative tinnitus ( $N=158)$
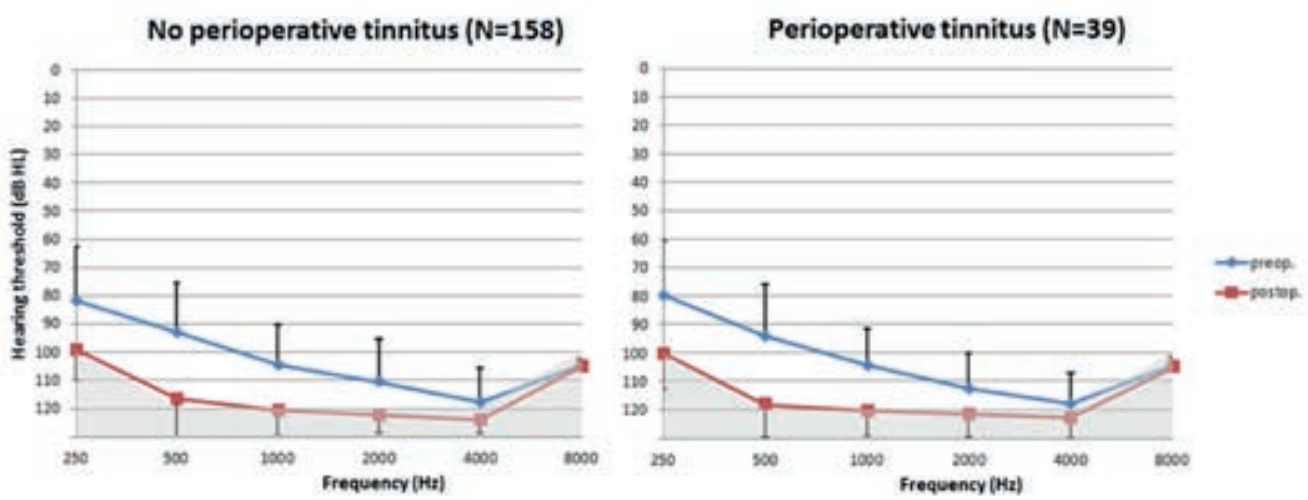

Figure 2: Averaged pre- and postoperative audiometric hearing thresholds. The left graph represents the "no perioperative tinnitus" group while the right graph represents the "perioperative tinnitus" group. Standard deviations were presented as error bars. Furthermore, the limits of the audiometer were represented in grey.

Three multivariable logistic regression models were constructed in order to examine the association of perioperative hearing deterioration with perioperative tinnitus after adjustment for gender and $\mathrm{Cl}$ brand and to test the potential interaction between perioperative deterioration of hearing thresholds and gender. Again, the implanted electrode array could not be included as a covariate due to the limited amount of cases with perioperative tinnitus. Model A shows a near-significant interaction between perioperative hearing deterioration and gender $(p=0.063)$. Although caution is required by interpreting this result as the number of variables used exceeds the recommended maximum number of variables (approximately $10 \%$ of the perioperative tinnitus cases found), for the sake of completeness, we studied the effect of stratifying by gender in Model B. After stratifying, the adjusted effect of perioperative hearing deterioration on perioperative tinnitus is not significant in both males and females. However, stratification resulted in small numbers of perioperative tinnitus cases in both males (22) and females (17). Therefore in Model C no stratification was applied and the interaction between perioperative deterioration of hearing thresholds and gender was omitted from the model. Again, no significant association was observed between perioperative hearing deterioration and perioperative tinnitus in a model corrected for gender and $\mathrm{Cl}$ brand.

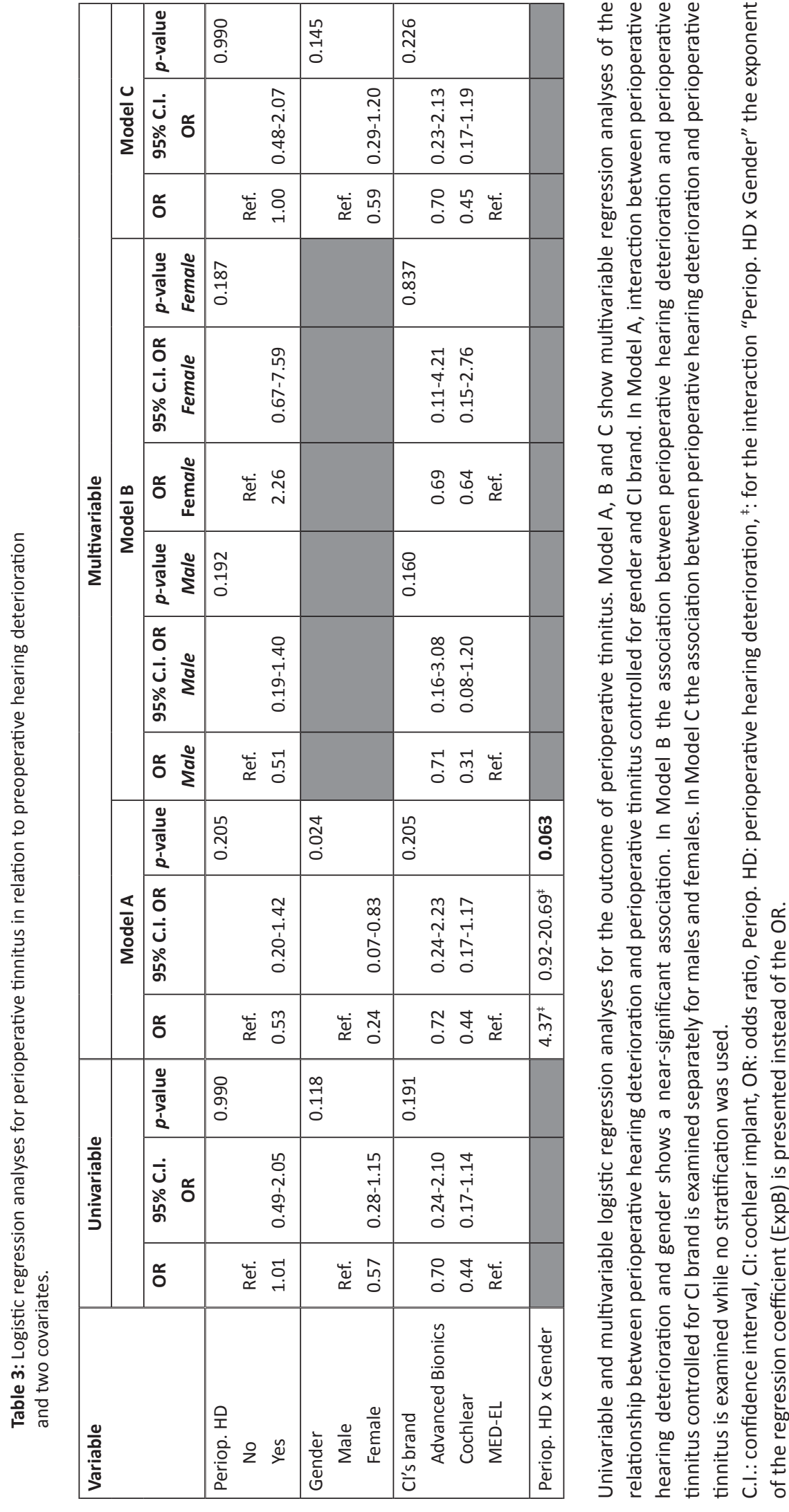


The average postoperative tinnitus loudness measured using a visual analogue scale (VAS) from 0 to 100 (see appendix; accessible via http://informahealthcare.com/loi/ija) was 52 (SD: 22.8) for the patient who newly developed tinnitus and 55 (SD: 18.2) for the patients with a deteriorated tinnitus following C surgery. Concerning TQ results, the average postoperative score was 38 (SD: 17.9) for the patients who newly developed tinnitus and 37 (SD: 18.3) for the patients with a deteriorated tinnitus following $\mathrm{Cl}$ surgery. These results related to the situation with an inactive $\mathrm{Cl}$. The original four TQ groups of tinnitus severity were combined into two groups to simplify analysis and obtain larger group size. Patients with mild to moderate TQ scores (i.e. TQ score<47) were assigned to the first group while patients with severe to very severe TQ scores (i.e. TQ score $\geq 47$ ) were assigned to the second group. Missing TQ scores were excluded from analysis. No association between TQ score and perioperative hearing deterioration was found as shown in Table $4(p=0.248)$

Table4: Association between hearing deterioration and tinnitusseverity related to the TQscore in the perioperative tinnitus group.

\begin{tabular}{|c|c|c|c|c|c|}
\hline & TQ: Mild-moderate & $\begin{array}{l}\text { TQ: Severe-very } \\
\text { severe }\end{array}$ & Missing & Total & \multirow{4}{*}{$\begin{array}{c}\text { OR: } 0.43 \\
95 \% \text { C.I. OR: } 0.09- \\
2.05 \\
\text { Exact p-value } \\
\text { (1-sided): } 0.248\end{array}$} \\
\hline No HD & $8(20.5 \%)$ & $5(12.8 \%)$ & $3(7.7 \%)$ & $16(41.0 \%)$ & \\
\hline HD & 15 (38.5\%) & $4(10.3 \%)$ & $4(10.3 \%)$ & 23 (59.0\%) & \\
\hline Total & $23(59.0 \%)$ & $9(23.1 \%)$ & $7(17.9 \%)$ & $39(100.0 \%)$ & \\
\hline
\end{tabular}

C.I.: confidence interval, HD: hearing deterioration, OR: odds ratio, TQ: Tinnitus Questionnaire

Finally, a binomial test was performed to check for a significant difference between the 5 -years tinnitus incidence of an age-matched general population sample without cochlear implantation and the percentage "perioperative tinnitus" in our study population. Despite slightly shorter follow up (the mean $\mathrm{Cl}$-duration was 4.7 years whereas the follow up of the age-matched general population was 5 years) the two populations are comparable. The difference between the $19.8 \%$ perioperative tinnitus in the $\mathrm{Cl}$ patients was not statistically significant from the $18.0 \% 5$-years incidence found in the epidemiologica study ( $p=0.564$ ) (Gopinath et al., 2010). Furthermore, the percentage perioperative tinnitus of the $\mathrm{Cl}$ recipients implanted with the Contour Advance electrode array, accounting for $44.2 \%$ of all electrode arrays implanted in the included patients and thus the most frequently implanted electrode array, is as is previously mentioned $18.4 \%$ (Table 1). Again, this percentage does not significantly deviate from the 5 -years tinnitus incidence in the age-matched general population described by Gopinath and colleagues ( $p>0.999$ ). Excluding the 66 patientsfromanalyses who experienced tinnitus pre-as well as postoperatively gives a tinnitus incidence of $19.1 \%$. The difference between the $19.1 \%$ tinnitus incidence in the $\mathrm{Cl}$-patients was again not significantly different from the $18.0 \%$ tinnitus incidence found in the above-mentioned epidemiological study $(p=0.880)$

\section{Discussion:}

The aim of this study was to investigate whether development of postoperative tinnitus or deterioration of already existing tinnitus may be associated to perioperative damage to the cochlea. Results show, as expected, that postoperative deterioration of hearing occurred significantly more often in the implanted ear compared to the contralateral ear. Furthermore, 25 out of 131 (19.1\%) patients newly developed tinnitus after $\mathrm{Cl}$ surgery and 14 out of $66(21.2 \%)$ patients who experienced pre- as well as postoperative tinnitus showed deterioration of their tinnitus by undergoing the $\mathrm{Cl}$ procedure. While some patients developed hearing deterioration and tinnitus after $\mathrm{Cl}$ surgery, there were others who had perioperative deterioration of hearing thresholds but no tinnitus. Moreover, a small group of patients even had perioperative tinnitus without having observed perioperative deterioration of hearing thresholds at all. Furthermore, the univariable and multivariable logistic regression analyses show no statistically significant association between perioperative deterioration of hearing thresholds and perioperative tinnitus nor between perioperative tinnitus and the covariates gender and Cl's brand (Table 3). Therefore, perioperative deterioration of hearing thresholds measured by PTA does not seem to be associated to the development or deterioration of tinnitus.

Because severe hearing loss is the main indication for cochlear implantation, hearing deterioration due to the surgery seems to be an unwanted but permissible side-effect, especially considering that the vast majority of patients will improve in hearing in everyday life when using their $\mathrm{Cl}$ (Alice et al., 2013). Applying our results to clinical practice, there appears to be no increased risk of developing chronic tinnitus after hearing deterioration due to cochlear implantation. No confounding effects were observed from gender and $\mathrm{Cl}$ brand.

The found tinnitus incidence following $\mathrm{Cl}$ surgery is comparable to previous studies (Webb et al., 1991; Greimel et al., 2003; Quaranta et al., 2004; Di Nardo et al., 2007; Postelmans et al., 2007; Akdogan et al. 2009; Pan et al., 2009; Hou et al., 2010; Kloostra et al., 2015). As far as known to the authors, this is the first study focused on a possible association between cochlear trauma due to $\mathrm{Cl}$ surgery, measured by PTA, and tinnitus.

The 5-years incidence in the general population, without cochlear implantation, within the same age group (18.0\%) (Gopinath et al., 2010) was not significantly different from the 4.7-years incidence for the Cl-patients (19.1\%) nor from the percentage perioperative tinnitus (19.8\%). Therefore, the current study found no significantly increased risk of developing or deterioration of tinnitus after cochlear implantation itself in the studied population. A considerable lower 5 -years incidence of tinnitus (5.7\%) was reported in Wisconsin among 3753 adults aged 48 to 92 years, with a mean age of 65.8 years (Nondahl et al., 2002). This lower 5 -years incidence can probably be explained by the relatively strict definition of 'significant' tinnitus used. Persons were classified as having tinnitus if the experienced tinnitus was at least moderate in severity or in case their tinnitus caused sleep problems.

Because tinnitus is associated with hearing loss (Langguth et al., 2013; Gopinath et al., 2010), one would expect that even without the $\mathrm{Cl}$ this hard of hearing population would have a higher incidence of tinnitus compared to an age-matched general population. Moreover, a similar incidence of tinnitus was also surprising because the current study showed that the $\mathrm{Cl}$-patients had an increased risk of hearin deterioration in the implanted ear after cochlear implantation.

\section{Limitations}

A limitation of the current study was the used patient group, consisting of conventional $\mathrm{Cl}$-candidates. Because the indication for $\mathrm{Cl}$ is severe bilateral hearing loss, this means that most included patients already have a severe hearing loss in the implanted ear, which complicates the detection of cochlear damage due to the surgery using PTA. With indication criteria for $\mathrm{Cl}$ shifting towards more residual hearing, patients with less hearing loss will in the future be more likely to be implanted, making it easie to consistently measure PTAs. Therefore, a prospective clinical study on subjects with substantially symmetrical residual hearing would be highly recommended. Furthermore, clinically measured PTA (octave frequencies) may not be the best measure for perioperative deterioration of hearing loss, 
because of the rather large between-frequency intervals. This means that, minor hearing loss at the non-tested frequencies could not be detected, but might possibly give rise to tinnitus. Testing a wider range of frequencies and inter-octave frequencies could improve data interpretation. Furthermore, ceiling effects are plausible due to the limits of the audiometer (Figure 2).

Another limitation is the condition itself. As tinnitus is a subjective symptom, researchers are dependent on the collaboration and honesty of their patients. Therefore the authors could not guarantee, however explicitly asked, that the patients answered the questions about their postoperative situation without bias from intracochlear electrical stimulation. This interaction is plausible as electrical stimulation of the auditory nerve has a known positive effect on tinnitus perception (Arts et al., 2015). However, because of residual inhibition (Osaki et al., 2005), the results obtained are possibly biased anyway. Therefore, to keep this retrospective study as accurate as possible, the analysis was driven by the above mentioned research question whereby, for example, the "no postoperative tinnitus" group was not further subdivided.

Furthermore, the time between implantation and questionnaire completion, which mount up to 10 years in this study, makes the accuracy of answers about preoperative tinnitus status questionable as patients might not be able to remember the situation before $\mathrm{Cl}$ surgery very well and may therefore induce recall bias (Kloostra et al., 2015). Nevertheless, a retrospective approach has also benefits compared to a prospective approach. A retrospective study design allows to use already existing clinical data as optimally as possible with relatively large numbers of subjects and, using this methodology, it avoids response shift (Kloostra et al., 2015). Response shift is defined as the phenomenon recognized by clinicians and researchers that people change their internal standards or values of how they function, with respect to their health-related quality of life (Schwartz et al., 2006), and it is assumed that $\mathrm{Cl}$ improves the healthrelated quality of life. Because two different situations at constant internal standards of how they function are questioned by means of the questionnaire, this type of bias was avoided. The current study has primarily clinical impact on patient counseling and informed consent; an additional prospective study design is required to reduce limitations as both approaches complement each other.

\section{Conclusion:}

It can be concluded that there is a small risk for deteriorated residual hearing after cochlear implantation. However, for patients with preoperative residual hearing, this deterioration of hearing loss does not seem to be associated with the development of postoperative chronic tinnitus. No confounding effects from gender or Cl's brand were observed. Finally, it can be concluded that, compared to an age-matched general population, patients undergoing cochlear implantation in the current study do not have a higher risk of developing tinnitus.

\section{Declaration of interest:}

The work of the first author (R.A.) was supported by a research grant from MED-EL Corporation, Innsbruck, Austria. The third author (M.J.) provided statistical support, made possible by a grant from the Dutch Heinsius Houbolt Foundation. The other authors declare no conflicts of interest. The authors alone are responsible for the content of this paper.

\section{References}

Adamchic, l., Tass, P.A., Langguth, B., Hauptmann, C., Koller, M., et al 2012. Linking the tinnitus questionnaire and the subjective clinical global impression: which differences are clinically mportant? Health and Quality of Life Outcomes, 10,79.

Akdogan, O., Ozcan, I., Ozbek, C., Dere, H. 2009. Tinnitus after cochlear implantation. Auris Nasus Larynx, 36, 210-212.

Alice, B., Silvia, M., Laura, G., Patrizia, T., Roberto, B. 2013. Cochlear implantation in the elderly: surgical and hearing outcomes. BMC Surg, 13(2),S1.

Amoodi, H.A., Mick, P.T., Shipp, D.B., Friesen, L.M., Nedzelski, J.M., et al 2011. The effects of unilateral cochlear implantation on the tinnitus handicap inventory and the influence on quality of life. Laryngoscope,121(7),1536-40.

Arts, R.A.G.J., George, E.L.J., Griessner, A., Zierhofer, C., Stokroos, R.J. 2015. Tinnitus suppression by intracochlear electrical stimulation in Single Sided Deafness - a prospective clinical trial: Part I. Audio Neurootol, 20(5), 294-313.

Arts, R.A., George, E.L., Stokroos, R.J., Vermeire, K. 2012. Review: cochlear implants as a treatment of tinnitus in single-sided deafness. Curr Opin Otolaryngol Head Neck Surg, 20(5),398-403.

Bauer, C.A., Wisner, K.W., Baizer, J.S., Brozoski, T.J. 2013. Tinnitus, Unipolar Brush Cells, and Cerebellar Glutamatergic Function in an Animal Model. PLoS One, 8(6), e64726.

Boothroyd, A., Cawkwell, S. 1970. Vibrotactile thresholds in pure tone audiometry. Acta Otolaryngol, 69(6),381-7

Demajumdar, R., Stoddart, R., Donaldson, I., Proops, D.W. 1999. Tinnitus, Cochlear implants and how they affect patients. J Laryngology Otology, 113,24-26.

Di Nardo, W., Cantore, I., Cianfrone, F., Melillo, P., Scorpecci, A., et al 2007. Tinnitus Modifications After cochlear implantation. Eur Arch Otorhinolaryngol , 264,1145-1149.

Eggermont, J.J., Roberts, L.E. 2012. The neuroscience of tinnitus: understanding abnormal and normal auditory perception. Front Syst Neurosci, 11,6:53.

Engdahl, B., Krog, N.H., Kvestad, E., Hoffman, H.J., Tambs, K. 2012. Occupation and the risk of bothersome tinnitus: results from a prospective cohort study (HUNT). BMJ Open, 21,2(1).

Erlandsson, S., Dauman, N. 2013. Categorization of tinnitus in view of history and medical discourse. Int J Qualitative Stud Health Well-being, 8,23530.

Gilles, A., Van Hal, G., De Ridder, D., Wouters, K., Van de Heyning, P. 2013. Epidemiology of noiseInduced tinnitus and the attitudes and beliefs towards noise and hearing protection in adolescents. PLoSOne, 24,8(7) 
Gopinath, B., McMahon, C.M., Rochtchina, E., Karpa, M.J., Mitchell, P. 2010. Incidence, persistence, and progression of tinnitus symptoms in older adults: the Blue Mountains Hearing Study. Ear Hear,31(3),407-12.

Greimel, K.V., Meco, C., Mair, A., Kohlböck, G., Albegger, K. 2003. Wie wird der Tinnitus durch eine Kochlearimplantation beeinflusst? HNO, 51, 226-231.

Hou, J-H., Zhao, S-P., Ning, F., Rao, S-Q., Han, D-Y. 2010. Postoperative complications in patients with Cochlear implants and impacts of nursing intervention. Acta Oto-Laryngologica, 130,687-695.

Jackson, P. 1985. A comparison of the effects of eighth nerve section with lidocaine on tinnitus. $J$ Laryngol Otol, 99(7),663-6.

Kloostra, F.J.J., Arnold, R., Hofman, R., Van Dijk, P. 2015. Changes in tinnitus after cochlear implantation and its relation with psychological functioning. Audiol Neurotol, 20,81-89.

Knipper, M., Van Dijk, P., Nunes, I., Rüttiger, L., Zimmermann, U. 2013. Advances in the neurobiology Of hearing disorders: recent developments regarding the basis of tinnitus and hyperacusis. Prog Neurobiol, 111,17-33.

Kompis, M., Pelizzone, M., Dillier, N., Allum, J., DeMin, N., et al 2012. Tinnitus before and 6 months after cochlear implantation. Audiol Neurotol, 17,161-168.

Langguth, B., Kreuzer, P.M., Kleinjung, T., De Ridder, D. 2002. Tinnitus: cause and clinical management. Lancet Neurol,12,920-30.

Lockwood, A.H., Salvi, R.J., Burkard, R.F. 2002. Tinnitus. N Eng/ J Med, 347,904-910.

Maes, I.H., Cima, R.F., Vlaeyen, J.W., Anteunis, L.J., Joore, M.A. 2013. Tinnitus: A Cost Study. Ear Hear, $34(4), 508-14$.

Meeus, O., Blaivie, C., Van de Heyning, P. 2007. Validation of the Dutch and the French version of the Tinnitus Questionnaire. B-ENT, 7,11-7.

Nondahl, D.M., Cruickshanks, K.J., Wiley, T.L., Klein, R., Klein, B.E.K., et al 2002. Prevalence and 5years incidence of tinnitus among older adults: The epidemiology of hearing loss study. J Am Acad Audiol, 13,323-331.

Olze, H., Szczepek, A.J., Haupt, H., Zirke, N., Graebel, S., et al 2012. The Implact of cochlear implantation on tinnitus, stress and quality of life in postlingually deafened patients. Audiol Neurotol, $17,2-11$.

Onafhankelijke Platform Cochleaire Implantatie (NL). Information for patients [internet]. OPCI (NL) 2005. [updated 2014 Apr 4; cited 2014 Apr 4]Available from:

http://www.opciweb.nl/over-ci/voor-wie/]
Osaki, Y, Nishimura, H., Takasawa, M., Imaizumi, M., Kawashima, T, et al 2005. Neural mechanism of residual inhibition of tinnitus in cochlear implant users. Neuroreport, 16(15),1625-8.

Pan, T., Tyler, R.S., Ji, H., Coelho, C., Gehringer, A.K., et al 2009. Changes in the tinnitus handicap questionnaire after cochlear implantation. Am J Audiol, 18(2),144-151.

Postelmans, J.T.F., Cleffken, B., Stokroos, R.J. 2007. Post-operative complications of cochlear implantation in adults and children: five years' experience in Maastricht. J Laryngology Otology, 121,318-323.

Quaranta, N., Wagstaff, S., Baguley, D.M. 2004. Tinnitus and cochlear implantation. Int J Audiol, $43,245-251$.

Schaaf, H., Eipp, C., Deubner, R., Hesse, G., Vasa, R., Gieler, U. 2009. [Psychosocial aspects of coping with tinnitus and psoriasis patients. A comparative study of suicidal tendencies, anxiety and depression]. [Article in German].HNO, 57(1),57-63.

Schaette, R., McAlpine, D. 2011. Tinnitus with a normal audiogram: physiological evidence for hidden hearing loss and computational model. J Neurosci, 31(38), 13452-7.

Schwartz, C.E., Bode, R., Repucci, N., Becker, J., Sprangers, M.A., et al 2006. The clinical significance of adaptation to changing health: a meta-analysis of response shift. Qual Life Res, 15,1533-1550.

Todt, I., Rademacher, G., Mutze, S., Ernst, A. 2014. Scalar electrode position and the occurrence of tinnitus in cochlear implantees. XI International Tinnitus Seminar, Berlin.

Todt, I., Rademacher, G., Mutze, S., Ramalingam, R., Wolter, S., et al 2015. Relationship between intracochlear electrode position and tinnitus in cochlear implantees. Acta Oto-Laryngologica (Epub ahead of print).

Turner, O., Windfuhr, K., Kapur, N. 2007. Suicide in deaf populations: a literature review. Ann Gen Psychiatry, 6,26.

Van de Heyning, P., Vermeire, K., Diebl, M., Nopp, P., Anderson, I., et al 2008. Incapacitating unilateral tinnitus in single-sided deafness treated by cochlear implantation. Ann Oto Rhinol Laryn, 117(9),645652.

Webb, R.L., Lehnhardt, E., Clark, G.M., Laszig, R., Pyman, B.C., et al 1991. Surgical complications with the cochlear multiple-channel intracochlear implant: experience at Hannover and Melbourne. Ann Otol Rhinol Laryngol, 100,131-136.

Zeman, F., Koller, M., Schecklmann, M., Langguth, B., Landgrebe, M. 2012. TRI database study group. Tinnitus assessment by means of standardized self-report questionnaires: psychometric properties of the Tinnitus Questionnaire (TQ), the Tinnitus Handicap Inventory (THI), and their short versions in an international and multi-lingual sample. Health Qual Life Outcomes, 18,10,128. 


\section{Appendix}

English translation of the used questionnaire.

Questionnaire Cl-patients:

1. What is your name?

2. What is your date of birth?

3. What is your gender?

O Male

4. On which side do you wear your Cochlear Implant (CI)?

O Left ear

5. Of which brand is your $\mathrm{Cl}$ ?

O Advanced Bionics

O Cochlear

O MED-EL

6. Did you experience tinnitus before the $\mathrm{Cl}$-surgery?
0 Yes, in my left ear
0 Yes, in my right ear
0 Yes, in both ears
O No

7. Do you experience now, after the Cl-surgery, tinnitus?
O Yes, in the implanted ear
O Yes, in the non-implanted ear
O Yes, in both ears
O No

All questions regarding tinnitus related to the situation after Cl-surgery, but when you are not using the $\mathrm{Cl}$

If you do not experience tinnitus after the Cl-surgery you don't have to answer the following questions!

Thank you for your cooperation!
8. In case you experienced tinnitus both before and after the $\mathrm{Cl}$-surgery; is the tinnitus loudness changed after the surgery?

O The tinnitus loudness is increased after the surgery

The tinnitus loudness is decreased after the surgery

O The tinnitus loudness did not change after the surgery

O N.A.

9. Is your tinnitus synchronous with the rhythm of your heartbeat?

0 Yes

O No

10. Is it possible for you to change your tinnitus by for example making some movements with your eyes or jaw?

O No

O Yes, how?

11. Please give the loudness of your tinnitus in the situation you are not using your $\mathrm{Cl}$ by marking the bar below. The left side represents 0: "no tinnitus" while the right side represents 100: "extremely loud". 100

(No Tinnitus)

(Extremely loud)

12. How would you describe your tinnitus?

0 The tinnitus has a low frequency (buzzing)

0 The tinnitus has an average frequency

0 The tinnitus has a high frequency (beeping)

13. Please could you additionally fill out the attached Tinnitus Questionnaire for the situation you are not using your $\mathrm{Cl}$ ?

14. If you have any comments related to the questionnaire, please add it below: 


\section{Chapter}

Remo A.G.J. Arts*

Erwin L.J. George,

Michelene N. Chenault

and Robert J. Stokroos

Department of ENT/Head and Neck Surgery,

Maastricht University Medical Centre,

PO Box 5800, 6202 AZ Maastricht,

The Netherlands

Ear Hear 2015;36:125-135 DOI:10.1097/ AUD.0000000000000090

iness cuts off from things,

'B1 indness cuts off from people'

but deafness cuts ofler (1905) He11en Kel1er (1905) 


\section{Abstract:}

Objectives: Research on tinnitus suppression by intracochlear electrical stimulation has gained interest over the past few decades and it has become easier to apply since the introduction of cochlear implants (CI). This study attempted to gain more insight into optimal stimulation characteristics for tinnitus suppression.

Design: Eleven subjects with unilateral $\mathrm{Cl}$ and tinnitus were recruited from our $\mathrm{Cl}$ clinic. Electrical stimulation, independent of acoustic sounds, was generated using their $\mathrm{Cl}$. The current prospective (single blinded) experimental study systematically assessed 2 stimulation parameters, namely current level and the anatomical stimulation site inside the cochlea, and their short-term effect on tinnitus.

Results: Approximately one-third of the tested conditions were successful in which case tinnitus loudness was reduced by at least $30 \%$. At least 1 successful condition was achieved for 9 subjects (82\%). Complete suppression was achieved in 6 out of 107 tested conditions (6\%).

The effect of subthreshold electrical stimulation on tinnitus suppression did not differ significantly from above threshold electrical stimulation. However, a positive relation between mean percentage tinnitus suppression and current level was observed. Pitch matched electrical stimulation did not appear to suppress tinnitus better than other tested conditions.

Conclusions:The majority of the subjectswereable to experience tinnitus reduction through intracochlear electrical stimulation independent of acoustic sounds. Tinnitus can be reduced with audible or even inaudible, subthreshold stimuli. Clear trends in optimal stimulation characteristics were not found. Optimal stimulus characteristics for tinnitus reduction therefore appear to be highly subject-specific.

\section{Short Summary}

This study was performed to gain more insight into optimal stimulation characteristics for tinnitus suppression. Intracochlear electrical stimulation, independent of acoustic sounds were generated in eleven subjects. The majority of subjects experienced tinnitus reduction and tinnitus could effectively be reduced in subjects who did not initially report tinnitus benefit when using their Cochlear Implant to perceive acoustic sounds. Tinnitus can be reduced by audible and inaudible stimuli, but optimal stimulus characteristics for tinnitus reduction seem to be highly subject-specific.

\section{Introduction:}

Tinnitus (aurium) is the perception of sound or noise in the absence of an external physical source. This disorder affects millions of people worldwide and severe forms are directly associated with psychiatric disorders including anxiety and depression (Luo et al. 2012). Its incidence is expected to increase due to the detrimental effects of occupational and leisure noise. Tinnitus has clinically been identified as a symptom of neurotological disease rather than a unitary complaint (Shulman 1979).

Although the pathophysiologic mechanism underlying tinnitus is still unclear, there is increasing evidence that auditory deprivation (not necessarily involving cochlear damage) can lead to subjective tinnitus. Tinnitus can e.g. be induced by simulated unilateral hearing loss (Schaette et al. 2012). Although not all individuals experiencing tinnitus have audiometric detectable hearing loss, those with hearing loss have an $83 \%$ greater chance of developing tinnitus (Nondahl et al. 2002). Tinnitus is partially or completely reversed about half of the time by hearing aid uptake (Surr et al. 1985) and up to $100 \%$ of the time by cochlear implants (CI) (Aschendorff et al. 1998; Kim et al. 2013). Cochlear implantation is also a viable treatment option for tinnitus arising from Single-Sided Deafness (SSD) (Bishop \& Eby 2009; Arts et al. 2012). Although research on tinnitus suppression by electrical stimulation of the cochlea has gained interest over the past few decades, it is not new. In the 18th century G.D. Wibel (1768) presented the possibility of tinnitus suppression by electrical stimulation. In 1893 Field claimed that tinnitus could completely be suppressed by electrical stimulation of the cochlea in humans (Cazals et al. 1977). This approach has been facilitated by the introduction of $\mathrm{Cl}$ which is a medical intervention that can restore sound perception to the severe-to-profound deaf via electrical stimulation of the auditory nerve (Fretz et al. 1985; Zeng 2004; Mielczarek et al. 2013).

Even though it appears that improving hearing can treat tinnitus, there is no consensus regarding the underlying mechanism responsible for tinnitus suppression. It can be asked whether tinnitus suppression is due to the reversal of responsible neural changes or that reduced tinnitus perception is caused by an attention shift from the tinnitus to sounds from the environment (less awareness of the tinnitus). The latter explanation is in line with the neurophysiologic model proposed by Jastreboff and Hazell (Jastreboff 1999). Previous studies show that tinnitus can at least in the short-term be suppressed by intracochlear electrical stimulation independent of acoustic sounds (Dauman et al. 1993; Rubinstein et al. 2003; Zeng et al. 2011; Chang et al. 2012) and even by stimuli that were not perceptible (Battmer et al. 1989). This stimulation differs from conventional $\mathrm{Cl}$-stimulation as it does not encode sounds from the environment.

These findings may lead to the development of a "Tinnitus Implant", an implantable device which generates intracochlear electrical stimulation to reverse neural changes as the underlying cause of tinnitus may be due to auditory deafferentiation. Essential for this application is that possible suppressive effects of electrical stimulation independent of acoustic sounds on tinnitus can be maintained over time, but the optimal stimulation parameters must be determined first. The theories on the cochlea as an ignition site for tinnitus together with the hypotheses regarding the way it can be influenced by electrical stimulation may indicate the need for individually modified parameters of electrical stimulation (Mielczarek et al. 2013).

To gain more insight into optimal intracochlear electrical stimulation characteristics for tinnitus suppression, the current prospective (single blinded) experimental study systematically assesses 2 stimulation parameters and their effects on tinnitus. The investigated parameters are 1. the applied 
current level and 2. the anatomical stimulation site inside the cochlea. It is hypothesized that subthreshold and thus inaudible current levels suppress tinnitus to a lesser extent than above threshold stimulation (Battmer et al. 1989; Chang et al. 2012). Furthermore, it is hypothesized that tinnitus suppression is optimal when applying pitch-matched electrical stimulation. Finally, it is hypothesized that noise band stimulation is superior to narrow band stimulation.

Besides effects on tinnitus reduction, the current study also investigated the effect of electrical stimulation on loudness adaptation. Loudness adaptation is defined as a decrease in perceived loudness for sustained, fixed-level stimulus, which from a patient's perspective can be beneficial when applyin stimulation in the long-term. Loudness is a subjective attribute of sound intensity which is also clearly affected by stimulus frequency and duration. A general consensus regarding the location in the auditor pathway of the mechanism responsible for loudness adaptation is lacking. Loudness adaptation might be due to the "restricted excitation pattern" in the cochlea (Scharf 1983) or by a peripherally based, retrocochlear mechanism (Javel 1996), or possibly by a central feedback mechanism (Tang et al. 2006). For normal hearing, the loudness of fixed level pure tones can decrease by $70-100 \%$ at $5 \mathrm{~dB}$ sensation level (SL), $20 \%$ at $40 \mathrm{~dB}$ SL but remain fairly constant at higher SLs (Hellman et al. 1997). Loudnes adaptation occurs mostly for high-frequency sounds and rarely for low-frequency sounds (Tang et al. 2006). Furthermore, at loudness intensities of $70 \mathrm{~dB}$ SPL or more, greater degrees of loudness adaptation are obtained in broad than in narrow stimulus bands (Carterette 1956)

\section{Materials and Methods:}

\section{Subjects}

Severe-to-profound hearing-impaired, cochlear implant users with postoperative chronic tinnitus, having their present $\mathrm{Cl}$ for at least 9 months, and being at least 18 years old, were screened from our tertiary referral otologic practice. Before enrollment, subjects completed the Tinnitus Handicap Inventory (THI) questionnaire (Newman et al. 1996) and a self-composed Tinnitus Characteristics questionnaire. The THI, which has been internationally validated, quantifies the effect of tinnitus on the patient's emotions and daily activities. Responders are asked to answer the questions with No (0 points) Sometimes (2 points) or Yes (4 points), with a higher THI-score indicating a more severe handicap. The Tinnitus Characteristics questionnaire consists of questions about etiology, tinnitus duration, -pitch and -loudness, the presence of tinnitus pre- and postoperative and the effect of Cl-use on tinnitus.

Inclusion criteria required that the subject's tinnitus be idiopathic, the tinnitus loudness be stable for at least 6 months and that tinnitus be at least mild which means that the loudness should be ranked 3 or more on a 0-10 Visual Analog Scale (VAS) and the THI-score be more than 16.

Six of the included subjects were male and 5 were female, with an average age of $60.1 \pm 6.4$ (SD) years ranging from 49 to 72 years. The duration of $\mathrm{Cl}$ use ranged from 3 to 9 years with an average of $5.0 \pm 1.9$ (SD) years. Five subjects used Advanced Bionics devices (Advanced Bionics LLC, Sylmar, CA, USA), 5 used Cochear (Cochlear Ltd., Sydney, Australia) and 1 subject used a MED-EL device (MED-EL Corporation, Innsbruck, Austria) (Table 1).

Tinnitus duration ranged from 2 to 40 years with an average of $15.1 \pm 14.4$ (SD) years. Average experienced tinnitus loudness on a VAS from 0-10 was $6.8 \pm 1.4$ (SD) ranging from 3.6 to 8.8 . Six subjects indicated that
Table 1: Patient demographics.

\begin{tabular}{cccccccc}
\hline Subject & Sex & $\begin{array}{c}\text { Age } \\
\text { (yr) }\end{array}$ & $\begin{array}{c}\text { Duration of deafness } \\
\text { implanted ear (yr) }\end{array}$ & Etiology & $\begin{array}{c}\text { Implanted } \\
\text { ear }\end{array}$ & $\begin{array}{c}\text { CI } \\
\text { manufacturer }\end{array}$ & $\begin{array}{c}\text { CI } \\
\text { duration } \\
\text { (yr) }\end{array}$ \\
\hline $\mathbf{1}$ & M & 69 & 12 & Unknown & Right & Cochlear & 3 \\
\hline $\mathbf{2}$ & M & 63 & 43 & Heritable & Right & Cochlear & 5 \\
\hline $\mathbf{3}$ & F & 49 & 44 & Otitis & Right & Cochlear & 6 \\
\hline $\mathbf{4}$ & F & 60 & 8 & Meningitis & Left & MED-EL & 3 \\
\hline $\mathbf{5}$ & M & 61 & 23 & Heritable & Left & Cochlear & 7 \\
\hline $\mathbf{6}$ & M & 61 & 26 & Sudden & Left & Cochlear & 6 \\
\hline $\mathbf{7}$ & F & 72 & Unknown & Unknown & Right & Advanced & 9 \\
\hline $\mathbf{8}$ & M & 57 & 57 & Congenital & Left & Advanced & 4 \\
\hline $\mathbf{9}$ & F & 57 & 57 & Meningitis & Right & Advanced & 3 \\
\hline $\mathbf{1 0}$ & F & 57 & 57 & Rhesus & Right & Advanced & 4 \\
& & & & factor & & Bionics & \\
\hline $\mathbf{1 1}$ & M & 55 & 7 & Cogan's & Right & Advanced & 5 \\
& & & & Syndrome & & Bionics & \\
\hline x- & & 60.1 & 33.4 & & & & 5.0 \\
\hline SD & & 6.4 & 20.7 & & & & 1.9 \\
\hline
\end{tabular}

$\mathrm{x}^{-}$, mean; SD, Standard Deviation

their tinnitus was stable independent of $\mathrm{Cl}$ activation or de-activation, 3 subjects indicated partial tinnitus suppression when their $\mathrm{Cl}$ was activated and 2 subjects indicated that their tinnitus was completely suppressed when their $\mathrm{Cl}$ was activated. Table 2 presents perceived tinnitus location and THI-scores per subject.

\section{Pitch match procedure}

Before the initiation of the experiments the tinnitus pitch matched electrode was identified by a twoAlternative Forced-Choice (2AFC) method. Subjects were presented with pairs of loudness balanced electric stimuli and asked to judge which stimulus most closely matched tinnitus pitch. Electric stimul consisted of trains of biphasic pulses presented at rates of at least 750 pulses per second (pps). Step size was 1 electrode. Each electrode was stimulated at least twice (2 "runs", apical-to-basal and basal-toapical, so that the range of starting frequency perception is highest and in order to prevent octaveconfusion). The pitch matched electrode was identified when the result of the first run was consistent with the result from the second run. Otherwise the whole procedure was repeated. Electrodes which were unusable for either technical or medical reasons were ignored during the pitch match procedure. 
Table 2: Tinnitus characteristics

\begin{tabular}{|c|c|c|c|c|c|c|c|}
\hline Subject & $\begin{array}{c}\text { Tinnitus } \\
\text { duration } \\
\text { (yr) }\end{array}$ & $\begin{array}{l}\text { Pre-op. } \\
\text { tinnitus }\end{array}$ & Tinnitus location & $\begin{array}{l}\text { THI } \\
\text { score }\end{array}$ & $\begin{array}{c}\text { Tinnitus } \\
\text { loudness } \\
(0-10)\end{array}$ & $\begin{array}{c}\text { Effect CI on } \\
\text { tinnitus } \\
\text { loudness }\end{array}$ & $\begin{array}{l}\text { PM electrode } \\
\text { (center freq.) }\end{array}$ \\
\hline 1 & 3 & + & Both ears $(L=R)$ & 8 & 6.2 & Clon $=$ Cloff & $13(1438 \mathrm{~Hz})$ \\
\hline 2 & $15-20$ & + & Both ears (L=R) & 34 & 6.9 & Clon -, Cloff + & $9(2501 \mathrm{~Hz})$ \\
\hline 3 & 2 & - & Implanted ear & 58 & 7.0 & Clon $<$ Cloff & $14(1251 \mathrm{~Hz})$ \\
\hline 4 & 8 & + & $\begin{array}{l}\text { Inside the head/ } \\
\text { implanted ear }\end{array}$ & 50 & $\begin{array}{l}\text { Depends on } \\
\text { fatigue }\end{array}$ & Clon -, Cloff + & $1(291 \mathrm{~Hz})$ \\
\hline 5 & 25 & + & $\begin{array}{l}\text { Inside the head/ } \\
\text { not implanted ear }\end{array}$ & 16 & 6.1 & Clon $=$ Cloff & $\begin{array}{c}<3(>5688 \\
\mathrm{Hz})^{*}\end{array}$ \\
\hline 6 & 40 & + & Both ears $(L>R)$ & 32 & 8.8 & Clon $=$ Cloff & $8(2876 \mathrm{~Hz})$ \\
\hline 7 & 9 & - & $\begin{array}{l}\text { Inside the head/ } \\
\text { not implanted ear }\end{array}$ & 80 & 7.1 & Clon $=$ Cloff & $13(3490 \mathrm{~Hz})$ \\
\hline 8 & 2 & - & $\begin{array}{l}\text { Inside the head/ } \\
\text { implanted ear }\end{array}$ & 10 & 3.6 & Clon $<$ Cloff & $1(336 \mathrm{~Hz})$ \\
\hline 9 & 3 & - & $\begin{array}{l}\text { Inside the head/ } \\
\text { implanted ear }\end{array}$ & 56 & 8.0 & Clon $=$ Cloff & $1(333 \mathrm{~Hz})$ \\
\hline 10 & 40 & + & $\begin{array}{l}\text { Inside the head/ } \\
\text { implanted ear }\end{array}$ & 32 & 6.7 & Clon = Cloff & $1(333 \mathrm{~Hz})$ \\
\hline 11 & 17 & + & $\begin{array}{l}\text { Inside the head/ } \\
\text { both ears }(L>R)\end{array}$ & 32 & 7.6 & Clon $<$ Cloff & $3(540 \mathrm{~Hz})$ \\
\hline $\mathrm{x}^{-}$ & 15.1 & & & 37.1 & 6.8 & & \\
\hline SD & 14.4 & & & 22.2 & 1.4 & & \\
\hline
\end{tabular}

Cloff=Cl switched off, $\mathrm{Clon}=\mathrm{Cl}$ switched on, $\mathrm{L}=$ left, $\mathrm{PM}=($ tinnitus) pitch matched, pre-op.=pre-operative $\mathrm{R}=$ right, $\mathrm{SD}=$ standard deviation, $\mathrm{THI}=$ Tinnitus Handicap Inventory, $\mathrm{x}^{-}=$mean. In the fourth column, $(L=R)$ means tinnitus equally dominates both ears and $(L>R)$ means tinnitus dominates left ear. In the second to last column the effect of $\mathrm{Cl}$ (speech perception) on tinnitus loudness is shown. Here, Clon=Cloff means no difference in tinnitus loudness between Cl switched on and Cl switched off. Clon-,Clofft means an absence of tinnitus when $\mathrm{Cl}$ is switched on, but a presence of tinnitus when $\mathrm{Cl}$ is de-activated. Furthermore, Clon<Cloff means louder tinnitus when $\mathrm{Cl}$ is de-activated compared to Cl activation. Asterisk: Subject 5 indicated that the tinnitus pitch was higher than the pitch generated by stimulation of electrode 3 . Because the more basal located electrodes were unusable due to VII nerve stimulation, we decided to use electrode 3 as pitch matched electrode.

\section{Intracochlear electrical stimulation for tinnitus reduction}

Intracochlear electrical stimulation was generated using the subject's $\mathrm{Cl}$. Standard clinical software was used to adjust the settings, based on the subject's clinical fit. An audio file uploaded on an mp3-player and connected to the processor was used to generate highly controlled charge-balanced, biphasic cathodic-first pulses. All stimuli were applied in monopolar mode. The audio file consisted of a steady state white noise. To control the current level, Thresholds (T-levels) and Maximum Comfortable Loudness levels (C-levels) were kept equal, giving a dynamic range of zero. Standard clinical settings were considered the default from which adjustments were made. Therefore, parameters as pulse rate and pulse duration were subject specific and depended on the subject's standard clinical settings.

Advanced Bionics (Advanced Bionics LLC, SyImar, CA, USA): Electrical stimuli were delivered through the Harmony speech processor. The mp3-player was connected to the processor by Auria Direct Connect, Auria Direct Connect Cable and BTE Audio Interface Cable. Furthermore, standard clinical software (SoundWave 2.1) and interface (Clarion Clinical Programming Interface) were used. HiRes-S was used and Fidelity 120 (and therefore Clearvoice) was disabled during this experiment. Input dynamic range was set on $30 \mathrm{~dB}$ and audio mix was set on "aux only".

Cochlear (Cochlear Ltd., Sydney, Australia): Electrical stimuli were delivered through the CP810 or Nucleus Freedom speech processor. A Nucleus Freedom Personal Audio Cable was used to connect the $\mathrm{mp3}$-player to the processor and standard clinical software (Custom Sound 3.2) and interface (Cochlear Ltd Programming Pod) were used. Smart sounds were disabled during these measurement sessions. Audio mixing ratio was set to " 0 " or " $\mathrm{A}$ " for the Nucleus Freedom or CP810 processor respectively (i.e. audio input only). Furthermore, the standard 'Advanced Combination Encoder' (ACE) strategy was modified to a strategy resembling 'Continuous Interleaved Sampling' (CIS). Here, the number of active electrodes equals (and was never higher than) the number of maxima ( $\mathrm{N}$-of-M). In this way a subset of the electrodes or channels is selected and these stimulation sites remain fixed so that the same subset of channels is stimulated during each frame. A minimum of disabled electrodes were evenly spread over the array.

MED-EL (MED-EL Corporation, Innsbruck, Austria): Electrical stimuli were delivered via the OPUS2 speech processor. An Adapter Cable was used to connect the mp3-player to the processor. Standard clinical software (Maestro System Software Version 4.1.1) and interface (Diagnostic Interface Box II) were used. In order to keep the pulse rate between channels fixed, the 'high-definition CIS' (HDCIS) strategy was used. Used audio mixing ratio was 50:50.

The present study consisted of 2 experiments. Each experiment investigated one single parameter. The effect of current level on tinnitus loudness was investigated in Experiment 1. All electrodes were enabled, except for Cochlear users in which a CIS-like strategy was applied or in case of disabled electrodes for medical/technical reasons. Used current levels were $10 \%$ of the clinical Dynamic Range (DR) subthreshold and $20 \%$, $50 \%$ - and $80 \%$ of the clinical DR above threshold. In addition, sham stimulation was applied to investigate placebo effects. Here, our subjects were asked to test a subthreshold stimulus which was inaudible, but in fact no stimulation was present. Conditions were offered in random order. Current level for each individual electrode was calculated on the basis of its clinical DR (Fig. 1). 


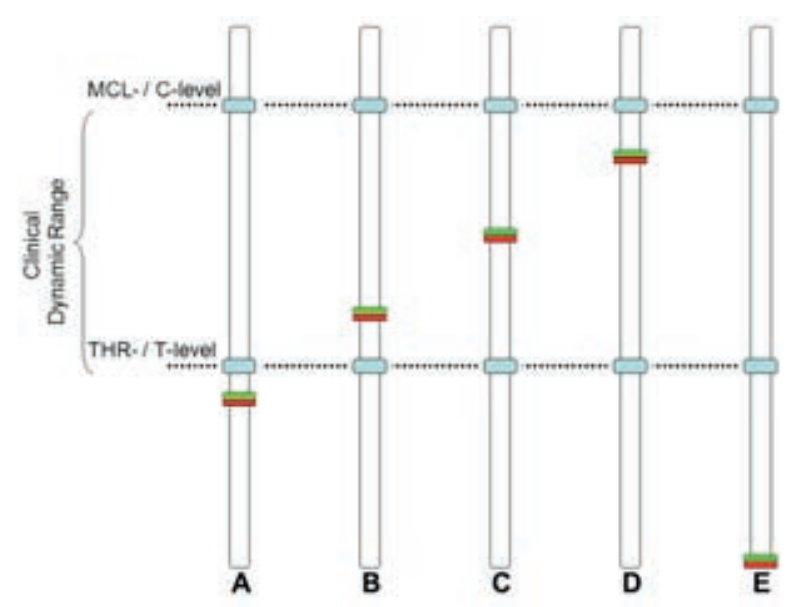

Figure 1: Schematic overview of applied current levels in Experiment 1. Condition A: current level set on 10\% subthreshold (-10\% DR). Condition B: current level set on $20 \%$ above threshold ( $20 \%$ DR). Condition C: current level set on $50 \%$ above threshold (50\% DR). Condition D: current level set on $80 \%$ above threshold ( $80 \%$ DR). Condition E: current level set on 0 current units (sham).

For Experiment 2, the parameter under investigation was the anatomical stimulation site inside the cochlea. In this experiment, current levels were fixed at levels giving optimal results with respect to tinnitus suppression and level of comfort in Experiment 1. Eight conditions were tested in random order: most apical electrode (1Ap), 3 most apical electrodes ( $3 \mathrm{Ap}$ ), central electrode (1Ce), central electrode together with the 2 adjacent electrodes (3Ce), most basal electrode (1Ba), 3 most basal electrodes (3Ba), electrode responsible for the tinnitus pitch (1PM, from now called "pitch matched electrode", Table 2) and pitch matched electrode together with the 2 nearest electrodes (3PM). The electrode configuration for each of the presented conditions per manufacturer is presented in Figure 2.

Both experiments were performed during one single visit to our clinic and took several hours, duration depending on residual inhibition (RI) and fatigue.

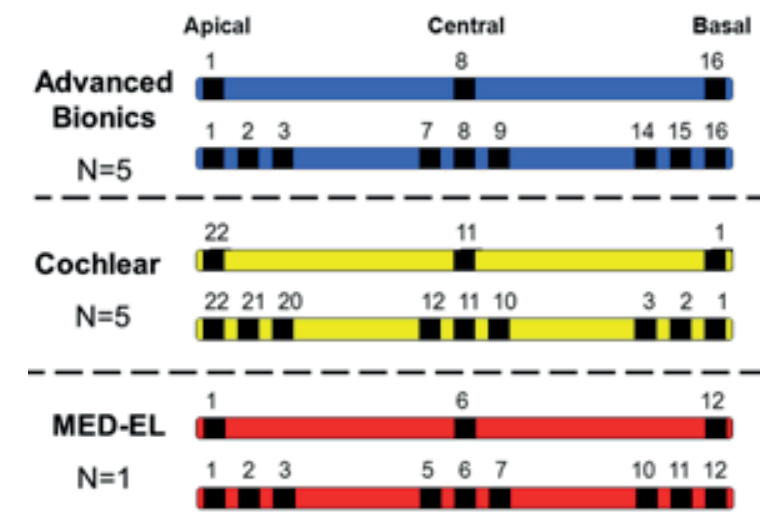

Figure 2: Electrode configurations for each of the presented conditions per manufacturer. The upper electrode array shows the activated electrode when narrow band stimulation was applied, the lower when noise band stimulation was applied. Pitch Matched electrical stimulation (not shown here) was based on the pitch of subject's tinnitus.

\section{Outcome measures}

Before each intervention and every 30 seconds during 5 minutes of stimulation, subjects were asked to rank the tinnitus loudness and the loudness perceived at that specific moment on the VAS. Subjects indicated loudness perception by setting a mark on a $10 \mathrm{~cm}$ bar, on which at the left ' 0 No Tinnitus' or ' 0 Not Audible' and at the right '10 Extreme Loud' was indicated (Kleine Punte et al. 2013). Tinnitus was allowed to return to baseline before testing the next condition, with a maximum recovery time of 30 minutes.

Intracochlear electrical stimulation effects on tinnitus were expressed in percentage tinnitus reduction, calculated using the following equation:

$$
\text { Tinnitus reduction }(\%)=\frac{L_{0}-L_{t}}{L_{0}} \times 100 \%
$$

Where, Lo represents baseline tinnitus loudness in absence of and before stimulation, ranking at time $t=$ $O$ (Cl switched off). $L t$ is the most extreme loudness ranking (minimum or maximum) at time $t$ using the VAS method. A value of $0 \%$ corresponds with no change in tinnitus while positive values indicate a reduction in tinnitus. A value of $100 \%$ indicates complete suppression. Negative values indicate an increase of tinnitus loudness. Tinnitus loudness changes of $30 \%$ or more were considered as clinically relevant and a percentage tinnitus reduction of $\geq 30 \%$ was considered as successful (Reavis et al. 2010; Chang et al. 2012).

\section{Loudness adaptatio}

The same equation, replacing tinnitus with stimulus loudness perception was used to evaluate loudness adaptation (Eq.1). Because the percentage suppression may be highlyaffected by the loudness perception at start, which is indicated as baseline, only data from Experiment 2 were observed, in which all conditions applied within subjects, had the same current level.

\section{Statistics}

In order to find the optimal parameters, in Experiment 1 different current levels while in Experiment 2 varying anatomical stimulation sites inside the cochlea were applied. Since the outcome measure considered was loudness perception relative to varying conditions per person, the non-parametric onesided Wilcoxon signed-rank tests for paired measurements or Paired Sample $t$ tests in case of a normal distribution (checked with the Kolmogorov-Smirnov test) were obtained. An independent Sample $t$ Test was performed for comparing outcomes between $\mathrm{Cl}$-subgroups. A $\chi^{2}$ test was obtained to compare successful conditions to unsuccessful conditions in subjects who responded to at least 1 stimulus. Statistics were performed with IBM SPSS Statistics, version 20, whereby $p$ values $<0.05$ were considered statistically significant.

\section{Ethical consideration}

This study was approved by the Ethics Committee of Maastricht University/academic hospital Maastricht in accordance with the declaration of Helsinki (approval No. NL38528.068.12). Written informed consent was obtained from each subject prior to participation. 


\section{Results:}

\section{General}

The Tinnitus Characteristics questionnaire indicated that for $52 \%$ of the patients in our clinic who experienced postoperative tinnitus, tinnitus loudness remained unchanged regardless of $\mathrm{Cl}$ activation or de-activation for standard clinical use (i.e. speech perception) while for $45 \%$ of the patients a positive effect of $\mathrm{Cl}$ use on tinnitus loudness was seen. A negative effect of $\mathrm{Cl}$ on tinnitus loudness was observed scarcely in 2 patients 1 of whom experienced tinnitus only when the $\mathrm{Cl}$ was activated. Here, it is unclear whether the sound perception is actually tinnitus or the perception is due to electrical stimulation by $\mathrm{Cl}$.

Both experiments were fully completed by $55 \%$ of the subjects (6/11). Subjects 3 and 4 did not start Experiment 2. Subject 3 decided to terminate the experiment prematurely due to fear of getting a headache during the first tested condition. Subject 4 dropped out during Experiment 1 due to a RI of more than half an hour. This subject did not return to complete the experiments because complete tinnitus suppression had also been achieved by using $\mathrm{Cl}$ for speech perception. Instructions were given to repea the experiment at home. This subject indicated that even at home the tinnitus could completely be suppressed with a subthreshold intracochlear electrical stimulation independent of acoustic sounds. Subjects 7 and 9 did not complete the ' $80 \%$ DR-condition' as this current level was perceived as being uncomfortably loud. Subject 7 also did not complete the last tested condition of Experiment 2 (3Ce) due to fatigue. Subject 6 did not complete the ' 1 Ba-condition' of Experiment 2 because stimulation remained imperceptible regardless of the amount of current suggesting the existence of a dead region.

Taking both experiments together, 107 conditions were tested of which 37 (35\%) were successful which means that tinnitus was reduced by $30 \%$ or more in about one-third of the conditions. In 6 conditions the tinnitus was completely suppressed. There were 2 tested conditions for which tinnitus loudness increased more than $30 \%$. Two of our tested subjects (18\%) described that tinnitus was completely suppressed in at least 1 tested condition. For $82 \%$ of the subjects (9/11) there was at least 1 successful condition. Details concerning suppression effects are shown below.

Subjects who responded to the first stimulus were more likely to respond to a second stimulus $\left(\chi^{2}=5.1, d\right.$ $=1, p=0.017$ ). Per subject response ranged from a minimum of $9 \%$ of conditions tested and up to $85 \%$ of tested conditions for an average of $41 \%$ of successful conditions (excluding subject 3 and 4 who dropped out). These results are comparable to those obtained by Chang et al. (2012).

\section{Experiment 1, the effect of current level on tinnitus suppression}

Four examples of tested conditions in Experiment 1 are given in Figure 3. Figure 3A shows an example of tinnitus reduction, combined with loudness adaptation. Figure $3 \mathrm{~B}$ shows the only tested condition in subject 3. After 3.5 minutes the subject terminated the experiment due to fear of headaches even when robust tinnitus suppression was indicated. Figure $3 \mathrm{C}$ is an example of complete tinnitus suppression by intracochlear electrical stimulation. Here, subthreshold electrical stimulation was applied on subject 4. After termination of this condition the tinnitus remained suppressed for more than half an hour and the remaining conditions, including the sham stimulation, could not be tested. For subject 10 when the full electrode array delivered a current level of $50 \%$ of the dynamic range, the tinnitus disappeared almost instantaneously (Fig. 3D). Here, the loudness perception of the stimulus was not lower than baseline tinnitus loudness and "masked" the tinnitus. Here the definition of masking is the process by which the threshold of audibility for tinnitus is raised by the presence of another (masking) perception. Although during the 5 minutes of stimulation the loudness of the stimulus remained more or less constant (there was no adaptation) the patient herself described the stimulus as beneficial, the stimulus being less annoying than the tinnitus.
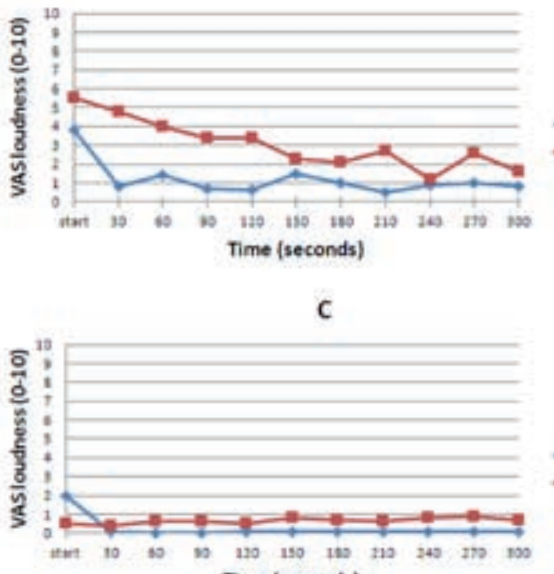

nime (seconds)
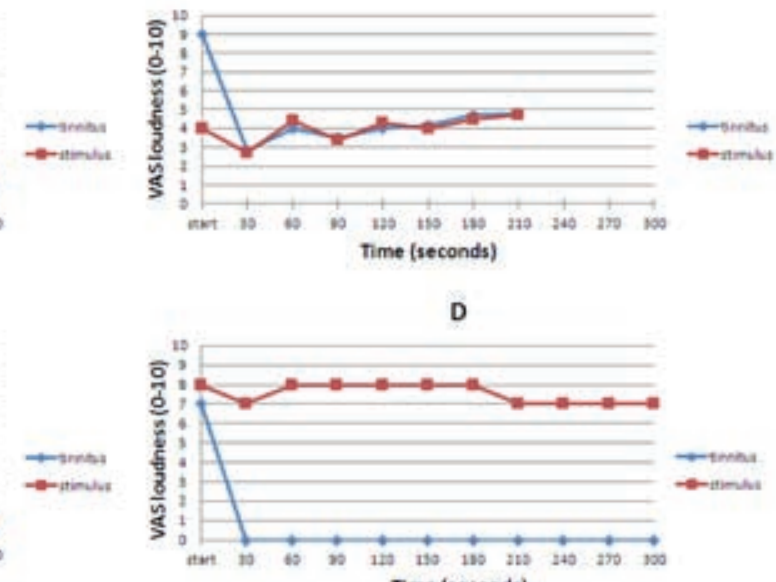

Time (neconds)
Figure 3: Examples of the effects of intracochlear electrical stimulation on tinnitus in Experiment 1. Each graph represents the loudness perception of both the tinnitus and the stimulus ranked on a Visual Analog Scale over time. A, $20 \%$ DR tested in subject 2. B, $50 \%$ DR tested in subject 3. C, $-10 \%$ DR tested in subject $4 . D, 50 \%$ DR tested in subject 10 .

The effect of current level on tinnitus is shown in Figure 4. Figure 4A shows the tinnitus reduction for different current levels for each subject, whereas Figure $4 B$ shows the tinnitus reduction per current level. Both graphs contain the same data. A $30 \%$ change in tinnitus loudness whether reduction or increase was considered clinically relevant.

Figure 4A shows that subject 1 and subject 11 did not respond to any of the broad band stimuli in this experiment (i.e. tinnitus was not reduced for $30 \%$ or more). For subject 11 , one condition $(20 \%$ DR) resulted in a tinnitus increase of $42 \%$. In this condition, the tinnitus loudness restored directly to baseline when stimulation ended. Although there were marked differences between individuals and the effect of current level, a positive relation between mean (average for the 11 subjects per condition) percentage tinnitus suppression and current level was observed (Fig. 4B). An average increase of 9.1 percentage points per $30 \%$ of the clinical DR was observed with a correlation coefficient between mean tinnitus suppression (in percentage) and current level (in percentage of the clinical DR) of 0.999 . 

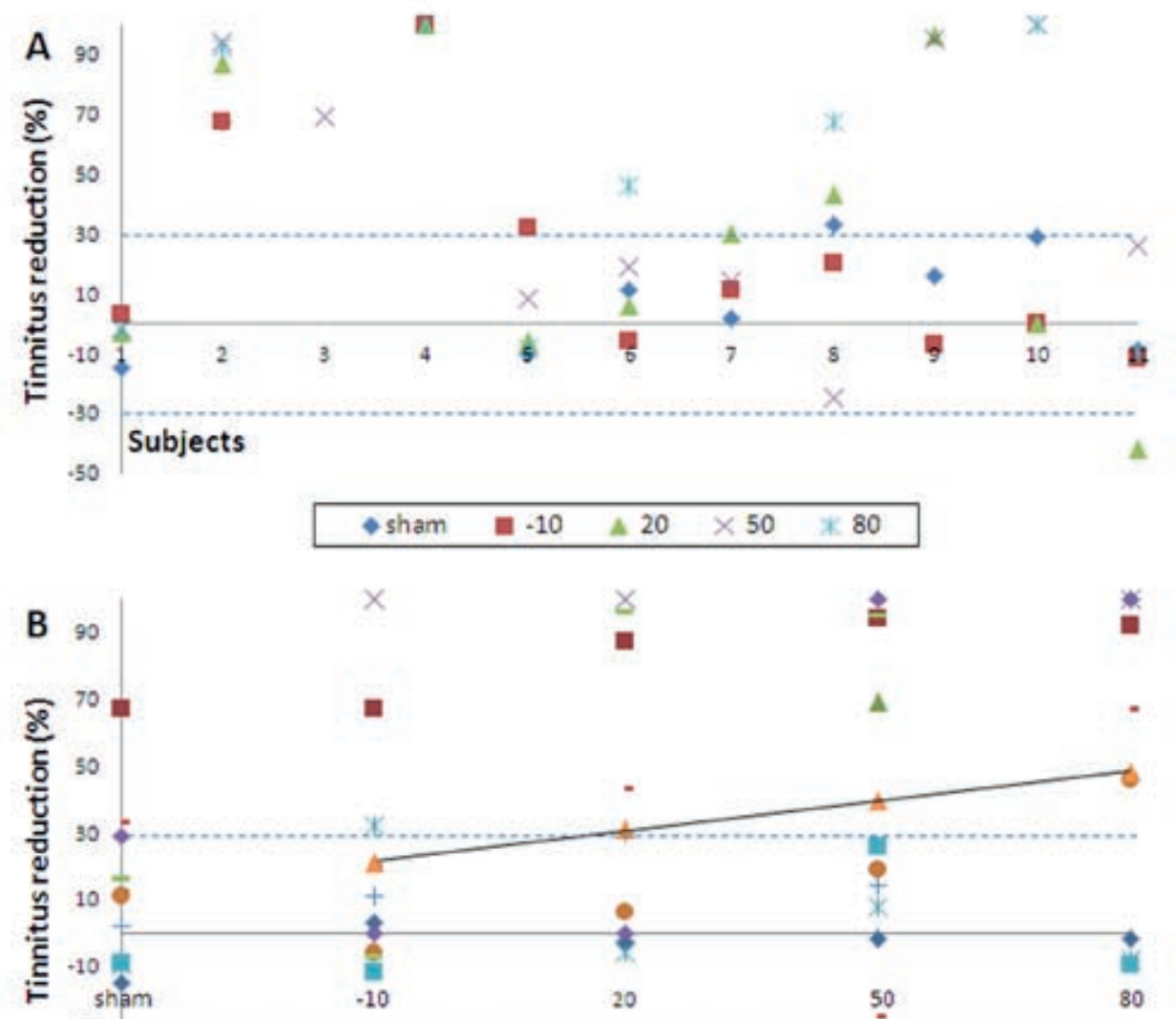

$\times$

๖

홈

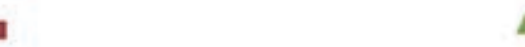

*

${ }^{50}{ }^{70}=$

.30

Current level (\% DR)

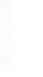

$\bullet \$ 1\|\$ 2 \Delta \$ 3 \times \$ 4 \times \$ 5 \bullet \$ 6+\$ 7-\$ 8-59 * \$ 10\| \$ 11 \triangle$ average

Figure 4: The effect of current level on tinnitus reduction shown per subject. Here, 'all' electrodes were stimulated. A, Tinnitus reduction for different current levels for each subject. B, Tinnitus reduction as a function of current Both graphs contain the same dat Tinnitus reduction and tinnitus increase of $30 \%$ or more was consideriod as clinically relevant.

Tinnitus reduction was successful in $22 \%(2 / 9)$ of tested sham stimulations. Subject 2 claimed a clearly reduced tinnitus (67\%) while for subject 8 a near-cutoff tinnitus reduction (33\%) was observed. Tinnitus reduction with sham stimulation ranged from $-15 \%$ to $67 \%$ with a median of $11 \%$ (Fig. 5A). Intracochlear electrical stimulation at the levels $50 \%$ and $80 \%$ of the clinical dynamic range gave rise to a larger percentage of tinnitus suppression compared to sham stimulation (Wilcoxon signed-ranks test, one sided $p=0.033$ and $p=0.014$ respectively). No significant differences between subthreshold electrical stimulation (-10\% DR) and suprathreshold electrical stimulation ( $20 \%$ DR, $50 \%$ DR or $80 \%$ DR) were obtained (Fig. 5A). There were near-significant differences between percentage of successful conditions in sham stimulation and above threshold stimulation with $20 \%$ DR and $80 \%$ DR (Wilcoxon signed-ranks test, one sided $p=0.079$ and $p=0.079$ respectively) (Fig. 5B).

A
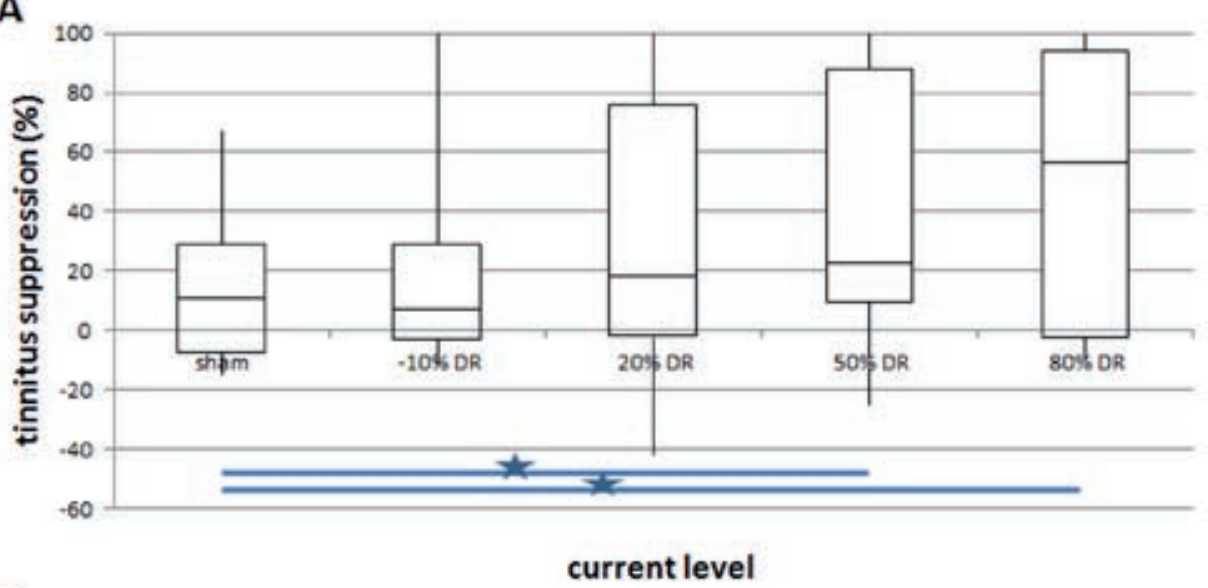

B

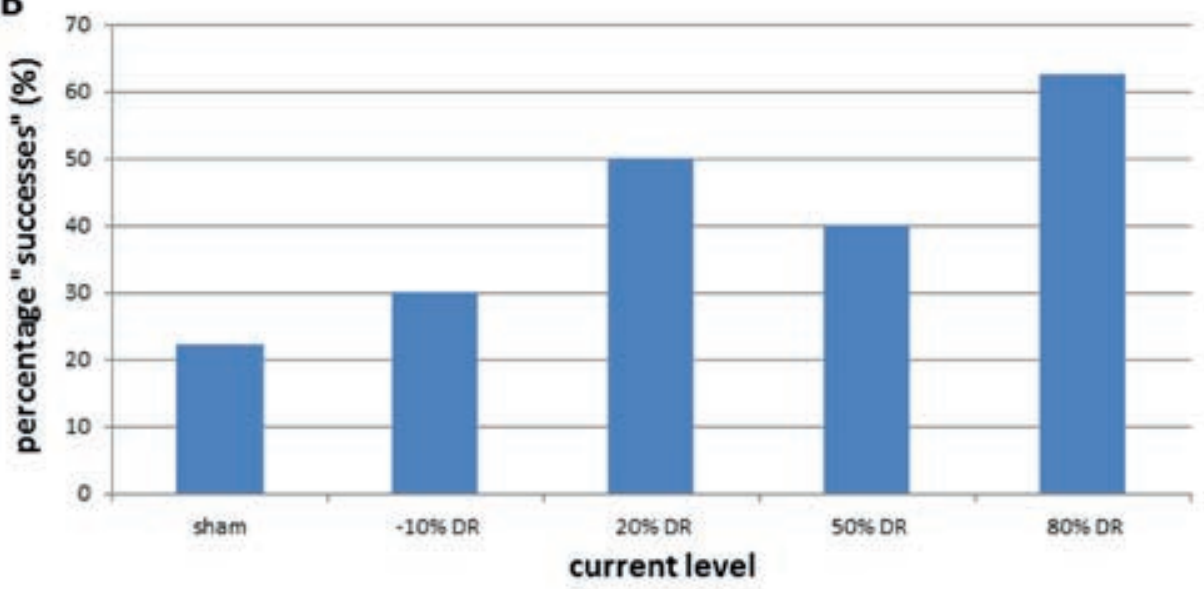

Figure 5: The effect of current level on tinnitus reduction shown for the sample. A, Boxplots indicating minimum, $25^{\text {th }}$ percentile, median, $75^{\text {th }}$ percentile and maximum values. Significant differences were obtained only between percentage tinnitus reduction in the sham stimulation vs. stimulation level of $50 \% \mathrm{DR}$ and vs. $80 \% \mathrm{DR}(\mathrm{p}=0.033$ and $p=0.014$ respectively). B, Percentage of successful conditions. There were no significant differences between any of the conditions.

\section{Experiment 2, the effect of anatomical stimulation site inside the cochlea on tinnitus reduction}

In Figure 6 four examples of the effect of intracochlear electrical stimulation on tinnitus in 2 of our subjects are given. The upper graphs pertain to subject 2 and the lower to subject 9 . Figure $6 \mathrm{~A}$ and Figure $6 \mathrm{~B}$ show tinnitus reduction using a stimulus which was almost inaudible or partly adapted to inaudible respectively. Figure $6 \mathrm{C}$ shows tinnitus reduction for approximately 1 minute while stimulus adapted to inaudible. Figure 6D shows a period of approximately 1 minute with complete tinnitus suppression. During this period the stimulus was adapted to inaudible. For clinical purposes this is a best-case scenario (total tinnitus suppression with loudness adaptation to the stimulus). This subject who had suffered tinnitus for 3 years was instantaneously freed of it (Table 2). After this period of complete silence tinnitus returned to its initial loudness level while adaptation continued. 
$A$

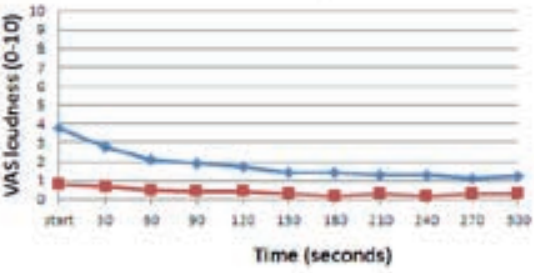

$$
\begin{array}{r}
\frac{5}{4} \\
\rightarrow-\min 4 \\
\frac{5}{5}
\end{array}
$$
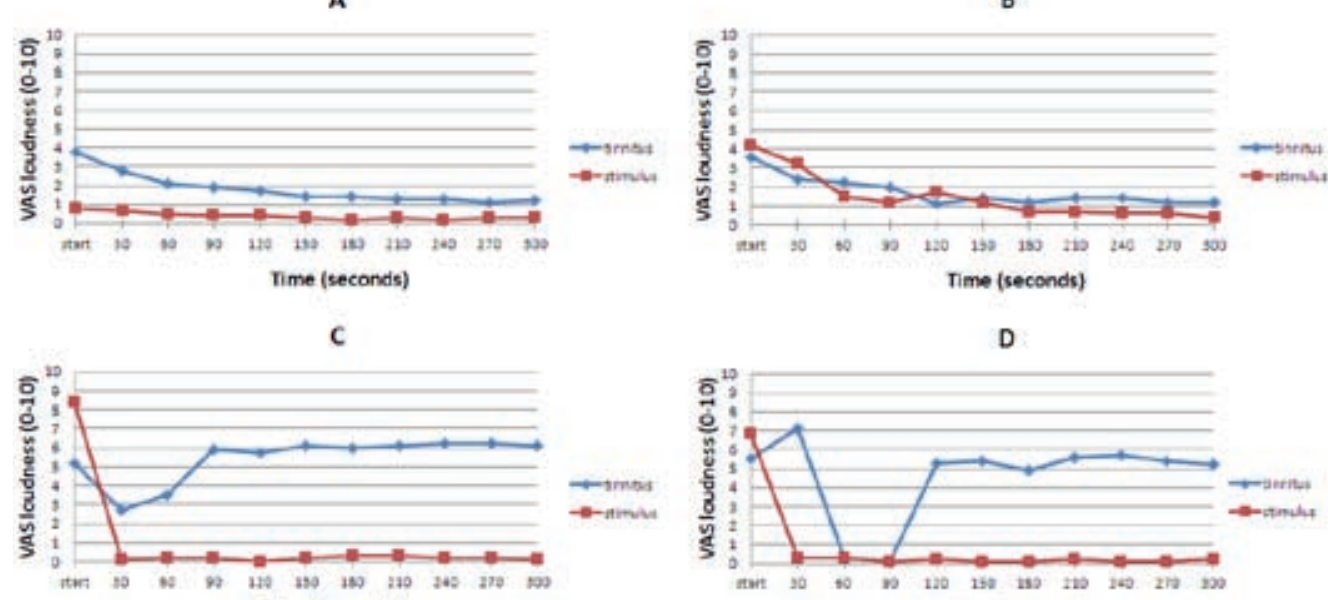

Time (seconds)

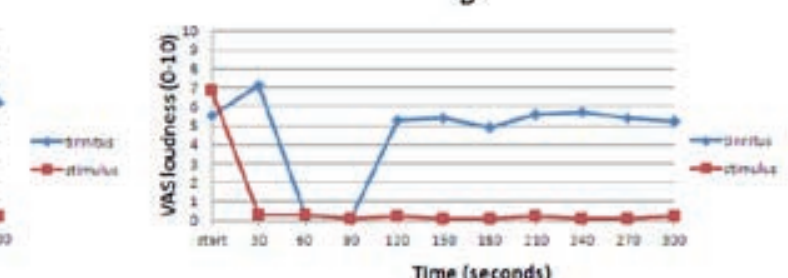

Time (seconds)

Figure 6: Examples of the effects of intracochlear electrical stimulation on tinnitus in Experiment 2. Each graph represents the loudness perception of both the tinnitus and the stimulus ranked on a Visual Analog Scale over time. The upper graphs represent the effect of intracochlear electrical stimulation on tinnitus in subject 2 with a current level of $20 \%$ DR. A, Narrow band apical (1 Ap). B, Narrow band central (1 Ce). The lower graphs represent the effect of intracochlear electrical stimulation on tinnitus in subject 9 with a current level of $20 \%$ DR. C, Noise band basal (3 Ba). D, Narrow band central (1 Ce).

The effect on anatomical stimulation sites inside the cochlea is shown in Figure 7, with tinnitus reduction per subject in Figure 7A and tinnitus reduction as a function of stimulation site in Figure 8B. Both graphs contain the same data. Two horizontal dotted lines in each graph mark the tinnitus reduction and tinnitus increase of $30 \%$ or more which is considered clinically relevant.

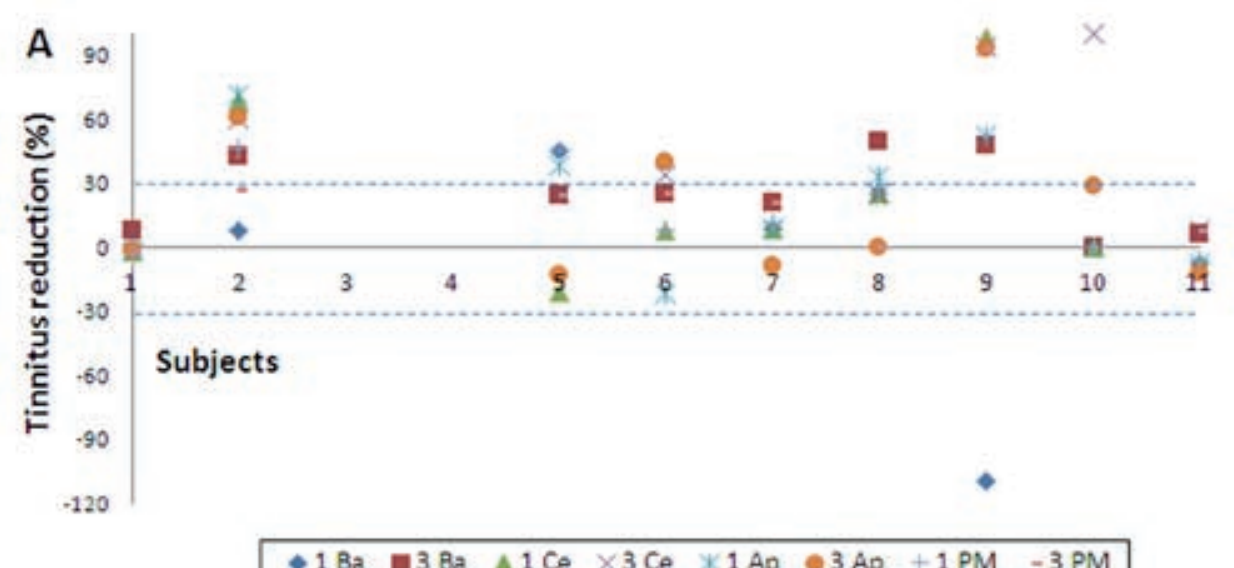

+ $1 \mathrm{Ba}$ a $3 \mathrm{Ba} \triangle 1 \mathrm{Ce} \times 3 \mathrm{Ce}$ *1 Ap $\bullet 3 \mathrm{Ap}+1 \mathrm{PM}-3 \mathrm{PM}$

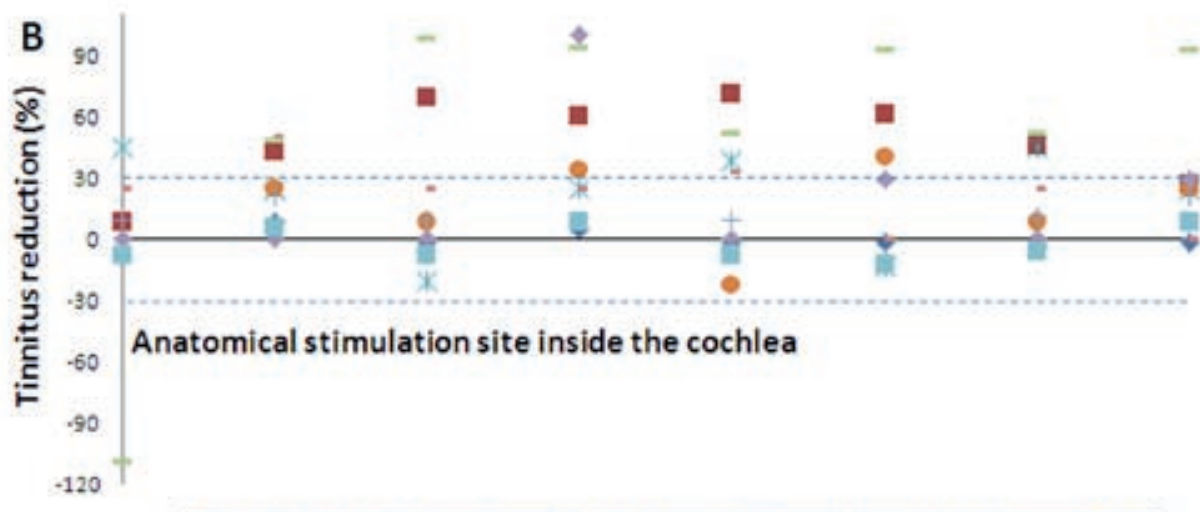

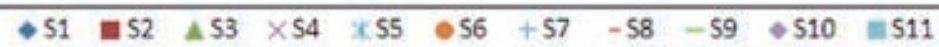

Figure 7: The effect of anatomical stimulation site inside the cochlea on tinnitus reduction. A, The tinnitus reduction for different stimulation sites per subject. B, Tinnitus reduction as a function of stimulation site. Conditions were basal ( $\mathrm{Ba}$ ), central (Ce), apical (Ap) or tinnitus pitch matched (PM). Each site was stimulated either narrow band (i.e. one single electrode) or noise band (i.e. 3 electrodes). Both graphs contain the same data.

In Figure 7A it can be seen that subjects 1,7 and 11 did not respond to any of the tested stimuli (i.e. tinnitus was not reduced for $30 \%$ or more). For subject 9 , one condition ( $1 \mathrm{Ba}$ ) resulted in doubling tinnitus loudness. In this condition, the tinnitus loudness decreased within minutes to baseline when stimulation was terminated. Data on subjects 3 and 4 are not available due to dropout.

In Figure 8 the group effects of stimulating different anatomical sites inside the cochlea on tinnitus are shown. The upper graphs represent boxplots of percentage tinnitus reduction. Central stimulation reduced tinnitus significantly better than pitch matched stimulation (Wilcoxon signed-rank test, one sided $p=0.043$ ) (Fig. 8B). The lower graphs present the percentage of successful conditions. Here, apical stimulation was significantly more successful than pitch-matched stimulation (Wilcoxon signed-rank test, one sided $p=0.042$ ) (Fig. 8D). 

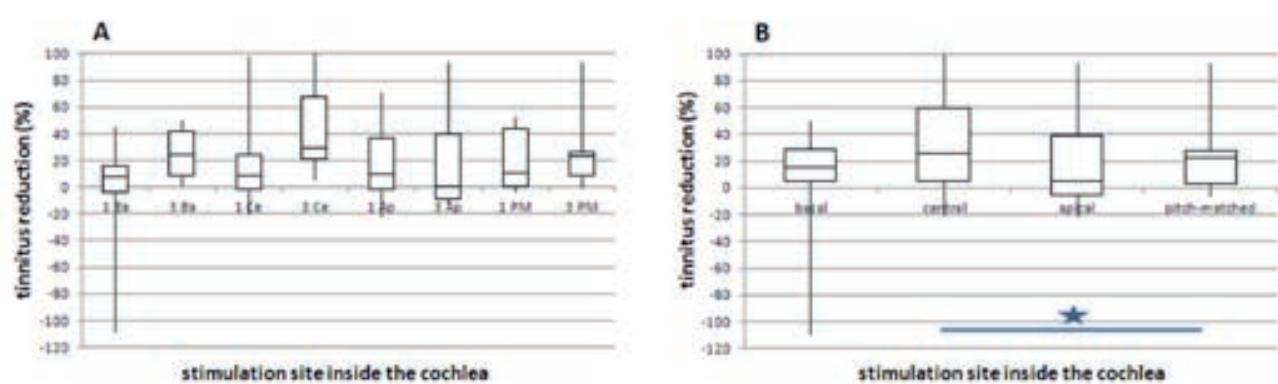

stimulation site inside the cochles
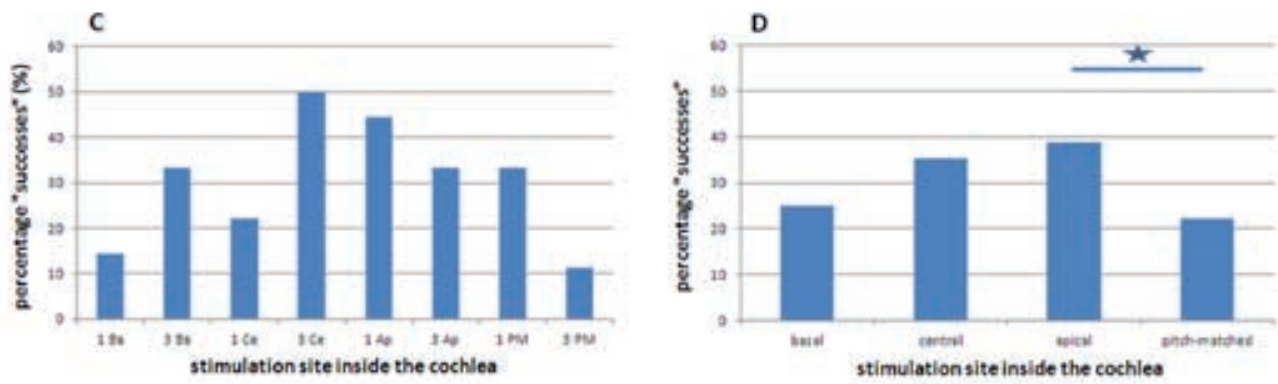

Figure 8: Sample stimulation effects of different stimulation sites inside the cochlea on tinnitus. The upper graphs (A and B) show minimum, $25^{\text {th }}$ percentile, median, $75^{\text {th }}$ percentile and maximum percentage tinnitus reduction for the different stimulation sites. A, Boxplots showing tinnitus reduction for the different (narrow or noise band) stimulation sites. B, The same data as is shown in graph A, now with merged narrow and noise band stimulation. Significant difference was obtained between pitch matched and central stimulation $(p=0.043)$. The lower graphs ( $C$ and $D$ ) representing the percentage of successful conditions in each tested condition. C, The percentage responders for the different (narrow or noise band) stimulation sites. D, The same data as is shown in graph C, now with merged narrow and noise band stimulation. Significant difference was obtained between pitch matched and apical stimulation $(p=0.042)$.

Finally, while we explored only short-term effects using steady-state electrical stimulation, subject 8 reported a long-term effect of intracochlear electrical stimulation on tinnitus. At the end of Experiment $2(1 \mathrm{Ap})$ there was a minor reduction which surprisingly was maintained for 4 full days.

\section{Loudness adaptation}

In Experiment 2, 10\% of the tested conditions (7/69) resulted in complete loudness adaptation. In 67\% of tested conditions $(46 / 69)$ the loudness decreased $30 \%$ or more, while loudness enhancement was reported in $14 \%$ of the tested conditions (10/69) of which $60 \%$ pertains to subject 1 . A comparison of the $A B$ and Cochlear groups showed that stimulation rate, pulse width and $\mathrm{Cl}$ manufacturer significantly affect loudness adaptation (Independent Sample $t$ Test, two sided, $p=0.011$ ). Our results cannot distinguish between stimulation rate, pulse width and manufacturer as Cochlear Cl's only tested low

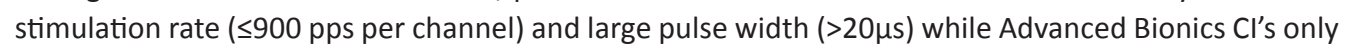
tested high stimulation rate ( $>2000 \mathrm{pps}$ per channel) and small pulse width $(<20 \mu \mathrm{s})$. Subject 4 who received a MED-EL Cl was 1 of our dropouts and could therefore not be included for data analysis. Concerning the effect of stimulus location, a significant lower loudness adaptation was observed in pitch-matched stimulation compared to apical, central or basal stimulation (Paired Sample $t$ Test, two sided, $p=0.041, p=0.020$ and $p<0.001$ respectively)

\section{Narrow vs. noise band stimulation}

Tinnitus reduction and loudness adaptation by narrow band (i.e. 1 electrode) pitch matched stimulation did not differ significantly from noise band (i.e. 3 adjacent electrodes) pitch matched stimulation (Fig. 8A) These results did not differ from merged (apical, central, basal and pitch-matched) narrow and noise band stimulation.

\section{Discussion:}

\section{Overall}

Tinnitus reduction could be obtained through intracochlear electrical stimulation independent of acoustic sounds for the majority of our subjects, which is in line with the findings of previous studies (Cazals et al. 1977; Battmer et al. 1989; Dauman et al. 1993; Hazell et al. 1993; Rubinstein et al. 2003; Zeng et al. 2011). In $82 \%$ of subjects (9/11) tinnitus was reduced for $30 \%$ or more for at least 1 of the tested conditions. Battmer et al. (1989) reported a tinnitus reduction by subthreshold electrical stimulation in $19 \%$ of their subjects (3/16) while Chang et al. (2012) reported tinnitus reduction by intracochlear electrical stimulation in $69 \%$ of their tested subjects $(9 / 13)$. Our results show that tinnitus could effectively be reduced in 5 subjects who did not initially report tinnitus benefit when using their $\mathrm{Cl}$ as a speech processor which is consistent with the findings of Zeng et al (2011) and Chang et al. (2012).

\section{Experiment 1}

It was hypothesized that subthreshold and thus theoretically inaudible current levels reduce tinnitus to a lesser extent than above threshold stimulation. We found no statistically significant evidence for this hypothesis although a trend was observed. While a current level of $10 \%$ of the clinical dynamic range below threshold (-10\% DR) should in theory be inaudible, stimulation was still perceived in 6 out of the 10 tested subjects, probably as a result of spectral loudness summation using broad band stimuli (Shannon 1983). Moreover spectral loudness summation may be the reason why 3 of 10 tested subjects declared that a current level of $80 \%$ of the clinical dynamic range $(80 \%$ DR) was uncomfortably loud so that the experiment could not be completed.

No clear level-dependence was observed for tinnitus reduction, which may be due to a large variability in tinnitus response between subjects and our small sample. Nevertheless medium and high current levels reduced tinnitus significantly better than sham stimulation and a positive correlation coefficient could be found between current level and the average percentage tinnitus reduction. These results conform partially to those by Chang et al. (2012) who reported that loud stimuli reduce tinnitus significantly better than soft sounds which may be (partially) be attributable to masking.

\section{Experiment 2}

It was hypothesized that tinnitus reduction is optimal when applying pitch-matched electrical stimulation. No evidence for this hypothesis was obtained. To our knowledge the studies assessing the effectiveness of pitch matched electrical stimulation are scarce and the studies that were performed were not able to draw conclusions on the effects of stimulation site possibly due to the limited number of subjects (Rubinstein et al. 2003; Rothholtz et al. 2009). Further research is needed, possibly in a larger group of participants, to evaluate the possible benefits of site-specific stimulation for tinnitus reduction. 
Our results regarding site-specific stimulation could be affected by the fact that subjects indicated that it was difficult to point out which electrode matched the pitch of the perceived tinnitus. None of the applied electrical stimuli perfectly matched the tinnitus pitch which underlines the fact that brain activity due to cochlear electrical stimulation differs from acoustic stimulation. It should also be considered that besides anatomical location, electrical stimulation rate and -level may also affect pitch perception (Pijls 1997; McKay et al. 2000; Landsberger et al. 2005; Carlyon et al. 2010).

Also a point of consideration is the fact that matching pitches between sounds with different perceptual qualities, as in our case of tinnitus pitch matching with electrical stimulation, are highly susceptible to non-sensory biases (Carlyon et al. 2010).

Other factors which may complicate tinnitus pitch matching are changes in the tinnitus pitch itself, perceived tinnitus consisting of multiple sounds and the difference in perceived location (tinnitus inside the head and stimulus outside the head). The present study tried to minimize influences of the first factor by testing on one single day assuming that tinnitus pitch would be stable for 1 day. Six out of 11 subjects (55\%) reported tinnitus consisting of multiple sounds. Here, in the pitch matching procedure the task was given to match the dominant tinnitus frequency. For the third factor mentioned, 2 subjects emphasized the difficulty they had in matching sound perceptions from different locations.

Caution is therefore required when interpreting our results as it is unclear whether the electrode found is a good representation of the tinnitus pitch. Presenting acoustic tones to an ear with residual hearing is probably a better method for psychoacoustic assessment (Vermeire et al. 2008; Carlyon et al. 2010). To our knowledge there is not yet a better method to determine the pitch-matched electrode in subjects with bilateral hearing loss treated with a $\mathrm{Cl}$ who suffer from postoperative tinnitus.

\section{Narrow vs. noise band stimulation}

Tinnitus reduction by narrow band (i.e. 1 electrode) pitch matched stimulation did not differ significantly from noise band (i.e. 3 adjacent electrodes) pitch matched stimulation. These results did not differ from merged (apical, central, basal and pitch-matched) narrow and noise band stimulation. Literature on the effect of noise band electrical stimulation on tinnitus is scarce. Kleine Punte et al. (2013) reported the necessity of full length cochlear stimulation for tinnitus suppression by electrical stimulation dependent of acoustic sounds (standard Cl-use). All of their tinnitus outcome measures remained unchanged with 1 , 2, 3 or 4 activated basal electrodes. Therefore, no difference was obtained between narrow band (i.e. 1 electrode) and noise band (i.e. 3 electrodes) stimulation on tinnitus reduction. These results are consistent with our findings. Further research is needed, possibly in a larger group of participants, to evaluate the possible benefits of noise band stimulation for tinnitus reduction.

\section{Predictors of effectiveness}

Subject 1 and 11 were non-responders in Experiment 1 while subject 1, 7 and 11 did not respond to any of the tested stimuli in Experiment 2. No predictions for effectiveness of intracochlear electrical stimulation on tinnitus suppression could be made considering the duration and cause of deafness (Table 1) or the duration of tinnitus (Table 2). This may partly be due to our small sample size. As can be seen in Table 1, the success of treating tinnitus by electrical stimulation may depend on the duration of deafness as the nonresponders had a relatively recent deafness.

\section{Loudness adaptation}

As seen in Figure 3 and Figure 6, loudness adaptation of the applied stimuli occurs regularly. From a clinical point of view it is interesting to note that tinnitus could remain reduced even when adaptation has occurred (Figs. 3A, 6B). Significantly lower loudness adaptation was observed in pitch-matched stimulation compared to apical, central, or basal stimulation, which may be due to difficulties in distinguishing between stimulus and tinnitus.

Tang et al. (2006) reported loudness adaptation in electric hearing in $5 \mathrm{Cl}$-recipients. Here, a great variability in subjects was observed which is possibly due to various etiologies and nerve survival patterns. Total loudness adaptation was observed in $22 \%$ of tested conditions, mostly in 1 subject which suggests a possible etiology of neuropathy. Stimulation rate, -place and-mode (monopolar or bipolar) did not appear to affect loudness adaptation. In contrast, Chang et al. (2012) reported significant effects of stimulation rate, -place and -level on loudness adaptation to electrical stimulation. These findings are in line with our results. To our knowledge there has been no reporting of the effect of electrical stimulation bandwidths on loudness adaptation. The differences between loudness adaptations in normal hearing versus electric hearing (using a $\mathrm{Cl}$ ) are possibly due to different loudness growth between different stimulus configurations.

\section{Masking vs. reduction}

Our findings show that tinnitus can be reduced with audible or even inaudible, subthreshold stimuli which is consistent with the study by Battmer et al.(1989). Complete tinnitus suppression induced by an inaudible stimulus would be the most convenient strategy for clinical application. Inaudible stimuli are subthreshold stimuli or stimuli with complete loudness adaptation. These stimulation characteristics represent the "code of silence" (Rubinstein et al. 2003). Complete silence was only experienced once by 1 patient for approximately 1 minute (Fig. 7D).

In reducing tinnitus complaints stimuli can either mask or effectively reduce the tinnitus. Masking diverts the patient's attention away from the tinnitus by competing sound perceptions while reduction is likely to modulate the activity of the auditory cortex and interrupt tinnitus generation. While it should be stressed that tinnitus reduction is more convenient for clinical application, masking could also be beneficial to alleviate tinnitus complaints (Fig. 3D).

\section{Potential weakness of the study}

Stimulation rate and pulse width are examples of parameters which were not under investigation and were therefore fixed per subject based on subject's clinical fit. These fixed parameters might be key factors for tinnitus reduction may muddle our results. An adjustment for these parameters could increase the interpretability of our results. Furthermore, stimulation rate and pulse width were not fixed between subjects and could decrease the comparability between subjects. As different $\mathrm{Cl}$ devices were used and current levels were calculated from the clinical DR, clinical settings including stimulation rate and pulse width were used as default from which variables under investigation were adjusted so that clinical DR remained unchanged. 
An additional study is currently being conducted which investigates in addition to current level and intracochlear anatomical location, the tinnitus suppressive effects of stimulation rate, amplitude modulation and polarity.

\section{Conclusion:}

Our results show that the majority of subjects were able to experience tinnitus reduction through intracochlear electrical stimulation independent of acoustic sounds. No robust trends could be found in optimal stimulation characteristics. No significant differences were obtained between subthreshold and above threshold electrical stimulation. Furthermore, statistically significant evidence was not found for the hypothesis that tinnitus reduction is optimal when applying pitch-matched electrical stimulation Instead, the optimal stimulus characteristics seem to be highly subject-specific. Therefore the ability to adjust stimulation characteristics using $\mathrm{Cl}$ seems to be indispensable requiring a customized, patientspecific treatment. This means that when using a $\mathrm{Cl}$ to manage tinnitus the clinical speech processor needs to be customized for optimal tinnitus reduction.

Before intracochlear electrical stimulation can be implemented as a potential tinnitus suppressor, longterm effects should be investigated. This treatment is only viable when reduction can be maintained over time. Given that our subjects are on the one hand bilaterally deaf and fitted with a unilateral $\mathrm{Cl}$ to improve hearing and on the other hand the applied intracochlear electrical stimulation to reduce tinnitus is independent of acoustic sounds, an investigation of the long-term effects is practically and ethically unfeasible. The authors are currently conducting a study on patients with unilateral hearing loss to test the long-term effects of intracochlear electrical stimulation independent of acoustic sounds to suppress tinnitus.

\section{Acknowledgements:}

The work of the first author (RA) was supported by a research grant from MED-EL Corporation, Innsbruck Austria. The third author (MC) provided statistical support, made possible by a grant from the Dutch Heinsius-Houbolt Foundation. The authors would like to thank two anonymous reviewers for their comments on an earlier draft of the manuscript.

\section{References:}

Arts, R.A., George, E.L., Stokroos, R.J., et al. (2012). Review: cochlear implants as a treatment of tinnitus in single-sided deafness. Curr Opin Otolaryngol Head Neck Surg. 20, 398-403.

Aschendorff, A., Pabst, G., Klenzner, T., et al. (1998). Tinnitus in cochlear implant users: the Freiburg experience. Int Tinnitus J. Vol.5 No.2, 162-164.

Battmer, R.D., Heermann, R., Laszig, R., (1989). Suppression of tinnitus by electric stimulation in cochlear implant patients. HNO. Vol. 37 No. 4: 148-52.

Bishop, C.E., \& Eby, T.L., (2010). The current status of audiologic rehabilitation for profound unilateral sensorineural hearing loss. Laryngoscope. 120:552-6.

Carlyon, R.P., Macherey, O., Frijns, J.H.M., et al. (2010). Pitch comparison between electrical stimulation of a cochlear implant and acoustic stimuli presented to a normal-hearing contralateral ear. J. Assoc. Res. Otolaryngol. 11,625-640.

Carterette, E.C. (1956). Loudness adaptation for bands of noise. J. Acoust. Soc. Am. Vol.28 Issue 5, 865871.

Cazals, Y., Negrevergine, M., Aran, J.-M., (1977). Electrical stimulation of the cochlea in man: hearing induction and tinnitus suppression. J Am Aud Soc. Vol.3 No.5.

Chang, JE., \& Zeng, FG., (2012). Tinnitus suppression by electrical stimulation of the auditory nerve. Front Syst Neurosci. Vol.6: Art.19.

Dauman, R., Tyler, R.S., Aran, J.M., (1993). Intracochlear electrical tinnitus reduction. Acta Otolaryngol (stockh). 113: 291-295

Fretz, R.J., \& Fravel, R.P., (1985). Design and function: a physical and electrical description of the 3M House cochlear implant system. Ear Hear 6(3suppl) :14S-19S.

Hazell, J.W.P., Jastreboff, P.J., Meerton, L.E., et al. (1993). Electrical tinnitus suppression: frequency dependence of effects. Int J Audiol. 32:68-77.

Hellman, R., Miskiewicz, A., Scharf, B. J., (1997). Loudness adaptation and excitation patterns: effects of frequency and level. Acoust. Soc. Am. 101:2176-2185.

Jastreboff, P.J. (1999). The neurophysiologic model of tinnitus and hyperacousis. In: Hazell, J. (Ed.), Proceedings of the sixth international tinnitus seminar. The tinnitus and hyperacusis Centre, London, pp.32-38,

Javel, F. J. (1996). Long-term adaptation in cat auditory-nerve fiber response. Acoust. Soc. Am. 99:10401052. 
Kim, D-K., Bae, S-C., Park, K-H., et al. (2013). Tinnitus in patients with profound hearing loss and the effect of cochlear implantation. Eur Arch Otorhinolaryngol.270:1803-1808.

Kleine Punte, A., De Ridder, D., Van de Heyning, P., (2013). On the necessity of full length electrical cochlear stimulation to suppress severe tinnitus in single-sided deafness. Hear Res. Vol. 295:24-29.

Landsberger, D.M., \& McKay, C.M., (2005). Perceptual differences between low and high rates of stimulation on single electrodes for cochlear implantees. J.Acoust. Soc. Am. 117:319-327.

Luo, H., Zhang, X., Nation, J., et al. (2012). Tinnitus suppression by electrical stimulation of the rat dorsal cochlear nucleus. Neurosci. Lett. 522:16-20

McKay, C.M., McDermott, H.J., Carlyon, R.P. (2000). Place and temporal cues in pitch perception: are they truly independent? Acoustic Research Letters Online, http://scitationaiporg/arlo/ 1:25-30.

Mielczarek, M., Konopka, W., Olszewski J., (2013). The application of direct current electrical stimulation of the ear and cervical spine kinesitherapy in tinnitus treatment. Auris Nasus Larynx. 40:61-65.

Newman, C.W., Jacobson, G. P., Spitzer, J.B., (1996). Development of the Tinnitus Handicap Inventory. Arch Otolaryngol Head Neck Surg. 122: 143-148.

Nondahl, D., Cruickshanks, K., Wiley, T., et al. (2002). Prevalence of 5-years incidence of tinnitus among older adults: the epidemiology of hearing loss study. J. Am. Acad. Audiol. 13, 323-331.

Pijl, S. (1997). Pulse rate matching by cochlear implant patients: effect of loudness randomization and electrode position. Ear Hear. 18:316-325.

Reavis, K.M., Chang, J.E., Zeng, F-G., (2010). Patterned sound therapy for the treatment of tinnitus. Hear J. Vol.63 No.11

Rothholtz, V.S., Tang, Q., Wu, E.C., et al. (2009). Exploring the parametric space of tinnitus suppression in a patient with a cochlear implant. Laryngoscope. 119:S117-S117.

Rubinstein, J.T., Tyler, R.S., Johnson, A., et al. (2003). Electrical suppression of tinnitus with high-rate pulse trains. Otol Neurotol. 24:478-485

Schaette, R., Turtle, C., Munro, K.J., (2012). Reversible induction of phantom auditory sensation through simulated unilateral hearing loss. PLoS ONE. Vol. 7 Issue 6, e35238.

Scharf, B. (1983). Loudness adaptation. JV Tobias, ED Schubert (Eds.), Hearing Research and Theory, Vol.2, Academic Press, New York, pp. 1-56.

Shannon, R.V. (1983). Multichannel electrical stimulation of the auditory nerve in man. II Channel interaction. Hear Res. 12:1-16.
Shulman, A. (1979). Tinnitus: Diagnosis/Treatment. Hear Aid. 6, 32-34.

Surr, R.K., Montgomery, A.A., Mueller, H.G., (1985). Effect of amplification on tinnitus among new hearing aid users. Ear Hear. 6, 71-75.

Tang, Q., Liu, S., Zeng, F-G., (2006). Loudness adaptation in acoustic and electric hearing. JARO-J Assoc Res Oto. Vol.7:59-70.

Vermeire, K., Nobbe, A., Schleich, P., et al. (2008). Neural tonotopy in cochlear implants: an evaluation in unilateral cochlear implant patients with unilateral deafness and tinnitus. Hear Res. 245:98-106.

Wibel, G.D. (1768). Casum aegroti auditu difficili zit. n. Stephens S.D.G., 1984. The treatment of tinnitus - a historical perceptive. J Laryngol Otol. 98:963-972.

Zeng, F-G. (2004). Trends in cochlear implants. Trends Amplif. Vol.8 No.1.

Zeng, F-G., Tang, Q., Dimitrijevic, A., et al. (2011). Tinnitus suppression by low-rate electric stimulation and its electrophysiological mechanisms. Hear Res. 277, 61-66. 


\section{Chapter}

Long-Term Effects of Intracochlear Electrical Stimulation with Looped Patterns on Tinnitus: A Case Study.

Remo A.G.J. Arts MSc. ${ }^{1}$

Erwin L.J. George PhD ${ }^{1}$,

Andreas Griessner $\mathrm{PhD}^{2}$

Clemens Zierhofer $\mathrm{PhD}^{2}$

Robert J. Stokroos $\mathrm{PhD}^{1}$

Department of ENT/Head and Neck

Surgery, Maastricht University Medical

Centre, Maastricht, The Netherlands

${ }^{2}$ Institute of Mechatronics, University of Innsbruck, Innsbruck, Austria

Accepted: Ear Nose Throat J

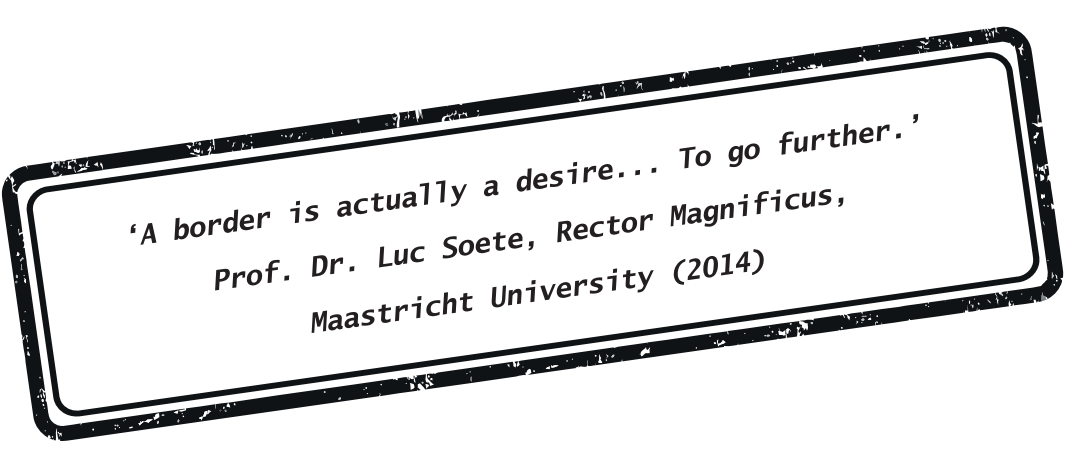




\section{Abstract:}

Electrical stimulation by Cochlear Implants (CI) has proven a viable treatment option for tinnitus in many recent studies and intracochlear electrical stimulation independent of an acoustic input seems to suppress tinnitus at least short term.

The present study investigated long-term effects of both standard clinical $\mathrm{Cl}$ and intracochlear electrica stimulation independent of an acoustic input on tinnitus in a single-sided deaf (SSD) subject with unilateral tinnitus.

Tinnitus can be reduced for months with both, standard clinical $\mathrm{Cl}$ and intracochlear electrical stimulation independent of an acoustic input. No negative effect of intracochlear electrical stimulation independen of an acoustic input on speech perception in noise was observed. Furthermore, the additional use of clinical $\mathrm{Cl}$ is advantageous for speech discrimination in our SSD subject.

Long-term tinnitus suppression can be achieved with intracochlear electrical stimulation with looped patterns. Results on speech discrimination in our SSD subject are consistent with previous studies.

\section{List of abbreviations:}

AH contralateral acoustic-hearing ear

AM Amplitude Modulation

$\mathrm{Cl} \quad$ Cochlear Implant

$\mathrm{HL}$ hearing loss

LIST Leuven Intelligibility Sentence Test

PM pitch matching

pps pulsesper second

PTA Pure Tone Average

RI Residual Inhibition

SL sensation leve

SRT Speech Reception Threshold

SSD Single Sided Deafness

TI Tinnitus Implant

TLM Tinnitus Loudness Matching

VAS Visual Analog Scale

3-AFC 3-alternative forced choice

\section{Introduction}

Subjective tinnitus (about $99 \%$ of all tinnitus cases) is the perception of sound in absence of an acoustic source. Tinnitus is a common symptom with a prevalence ranging from 10\%-15\% [1] and an increasing incidence [2]. It is a major global burden associated with excessive exposure to leisure noise [3] stress and hearing impairment [4-6]. Tinnitus may cause many issues ranging from hearing and attention deficits to depression and even suicide [7]. Although the precise underlying mechanism is still under debate, it commonly results from auditory deprivation or deafferentation $[8,9]$, analogous to phantom pain after somatosensory deprivation [10] and the perception of phosphenes after visual deprivation [11,12].

There is little doubt that tinnitus has a central origin resulting from neural homeostatic plasticity which pathologically increases response gain. Any lesion or abnormality of the auditory pathway from the cochlea to the cortex altering its normal function can cause subjective tinnitus, even when the audiogram is normal $[9,13,14]$. When auditory deprivation is caused at the periphery, treatment efforts are generally focused on the origin of the deafferentiation. Examples are conventional hearing aids [15] or Cochlear Implants which have recently been under investigation as a treatment option for tinnitus [16], even in Single Sided Deafness (SSD) [17-19]. Cochlear implants are implantable electronic hearing aids for hearing restoration in the profoundly deaf which have been in clinical use since the early 1980s [20].

Applying cochlear implants for alleviating tinnitus complaints may be a feasible approach: the plasticity which underlies tinnitus can probably be reversed by restoring auditory signal processing. Prior studies have shown that tinnitus can be suppressed for several minutes with intracochlear electrical stimulation independent of an acoustic input [7,21,22], but there is a lack of reports on long-term effects on tinnitus. Here, we present our findings of an SSD subject who received a "Tinnitus Implant" (TI), a cochlear implant specially implanted for the purpose of tinnitus suppression, in this long-term trial. The TI generates simple intracochlear electrical stimulation (i.e. intracochlear electrical stimulation independent of an acoustic input by continuously looping simple buffered pulse patterns and thus does not make use of the external microphone signal). This patient experiences robust long-term tinnitus reduction. To our knowledge this is the first report of long-term effects of intracochlear electrical stimulation, independent of an acoustic input, on tinnitus.

\section{Case report}

\section{1. Subject}

A 53-year-old male presented with a two-year history of debilitating tinnitus in the right ear following an idiopathic profound sudden sensorineural hearing loss in this right ear with a Pure Tone Average (PTA averaged across $0.5,1$ and $2 \mathrm{kHz}$ ) of $97 \mathrm{~dB} \mathrm{HL}$. In his left ear he had pure tone thresholds better than $20 \mathrm{~dB}$ $\mathrm{HL}$ for low frequencies $(0.25-1 \mathrm{kHz}$ in octave steps) and a mild sensorineural hearing loss in the high frequencies (2-8 kHz in octave steps) with a PTA of $18 \mathrm{~dB} \mathrm{HL}$. His tinnitus was perceived continuously and was described as "the sound of the sea to the sound of crickets". He suffered more from the tinnitus than from his hearing impairment due to the sudden single-sided deafness. Prior to implantation no treatment options were tried as a hearing aid or masking device would be unsuccessful due to his deafness. The patient subjectively rated his tinnitus loudness as 7 on a 0-10 Visual Analog Scale (VAS). He was able to match the tinnitus in his deaf right ear to a white noise with a loudness of $62 \mathrm{~dB}$ sensation level (SL) in his normal left ear offered by headphone. 


\section{2. Tinnitus Implant}

In order to control his tinnitus the patient underwent implantation of a CONCERTO Cl with a Flex28 electrode array (MED-EL Corporation, Innsbruck, Austria) using the mastoidectomy with posterio tympanotomy approach. Insertion occurred via the round window membrane. An OPUS2 speech processor (MED-EL Corporation, Innsbruck, Austria) was used which was converted into a TI pulse generator using customized software (Inst. of Mechatronics, Innsbruck, Austria) in Matlab version 7.11.0 (R2010b) (The Mathworks, Natick, MA, USA). The TI was used to generate continuous charge-balanced, biphasic pulses in monopolar mode.

\section{3. Follow up}

Baseline measurements were performed prior to surgery. Measurements were repeated approximately one week post-operatively to assess surgical effects on tinnitus, and at predetermined moments during the follow-up (Fig.1). Cl activation occurred approximately one month post-operatively, after which conventional $\mathrm{Cl}$ rehabilitation was performed for approximately two months to reach stable loudness perceptions of electrical stimulation. Subsequently, the $\mathrm{Cl}$ was deactivated for one week to reverse the possible influence of providing a hearing sensation on tinnitus.

Then, simple electrical stimulation was generated with the TI (i.e. the converted $\mathrm{Cl}$ ) in order to find the optimal pattern that provides tinnitus suppression. During this fine tuning procedure, stimulus location, stimulation rate, amplitude, amplitude modulation (AM) and polarity (anodic first or cathodic first) were systematically varied in random order. This procedure was conducted in two 6 -hour visits. During these visits, 48 stimulation patterns were examined for five minutes each, scoring them on perceived tinnitus and stimulus loudness.

After finding stimulation patterns which seemed to be optimal for short term tinnitus reduction, sham stimulation was applied for one week to investigate placebo effects. Here, our subject was informed that a subthreshold and thus inaudible stimulus was tested, but in fact no stimulation was present. Subsequent to the fine tuning procedure, the four most effective stimulation patterns obtained were activated for two full days each. At the end of each day in the two-day periods, the subject scored the pattern on tinnitus reduction, stimulus loudness and comfort. Finally, the most optimal stimulus as regards tinnitus reduction and comfort, based on patient's experiences, was applied for three full months in order to assess the long-term effects of simple electrical stimulation on tinnitus.

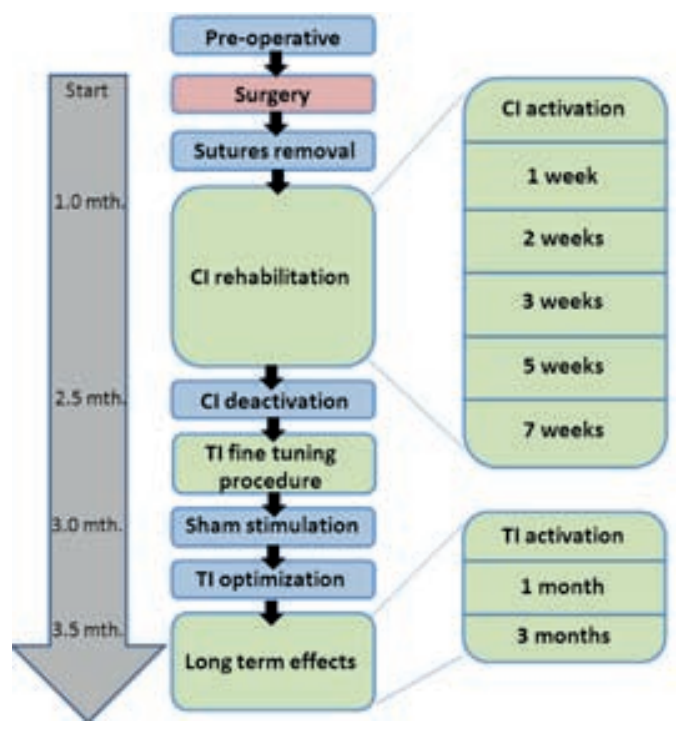

Figure 1: Flowchart of the applied protocol. Shown on the very right: The points in time when measurements were performed during $\mathrm{CI}$ rehabilitation and testing the long-term effects of $\mathrm{TI}$.

\section{4. Outcome measures}

2. 4.1.Tinnitus

Tinnitus pitch and loudness were measured, under headphones (Telephonics, TDH-39P), via the contralateral Acoustic-Hearingear (AH). Tinnitus Pitch Matching(PM) was performed using a 3-Alternative Forced Choice (3-AFC) method. Pure tones, $1 / 3$ octave narrow band noises and warble tones within the $250-8000 \mathrm{~Hz}$ range and white noise were offered. Tinnitus Loudness Matching (TLM) was measured in $\mathrm{dB}$ SL using predetermined tinnitus pitch. Loudness steps of $1 \mathrm{~dB}$ were used in a "one up two down" adaptive staircase rule.

For the VAS method, the subject marked how he perceived tinnitus loudness, amount of discomfort, effect on life and extent of problems due to tinnitus, respectively, on a $10 \mathrm{~cm}$ bar. At the left on this bar ' 0 No Tinnitus', '0 No Discomfort', '0 No effect on Life' or '0 No Problems' and at the right' 10 Extremely Loud', '10 Extremely Burdensome','10 Maximal Effect' or'10Extremely Problematic' wereindicated, respectively [23].

\section{4.2. Speech perception}

Speech discrimination scores in quiet were tested using open-set Dutch monosyllable words (ConsonantVowel-Consonant) in a free-field-condition in a sound-treated booth. In order to determine the effect of the standard $\mathrm{Cl}$ on speech perception, the $\mathrm{AH}$ was shielded using an earplug (E-A-Rsoft $\left.{ }^{\mathrm{TM}} \mathrm{FX}\right)$ together with an earmuff (E-A-R Ultra 9000). Furthermore, speech discrimination scores were additionally obtained using the Cl's direct input to eliminate the AH completely. For this purpose, an Adapter Cable (EXT-modus) was used to connect an mp3-player to the processor.

Speech recognition in noise was tested using the Leuven Intelligibility Sentence Test (LIST) [24] in an adaptive procedure. Tests were performed in a free-field-condition in a sound-treated booth. The subject was seated one meter away from the loudspeakers, with one at the front of the patient and the other to 
the right. Loudspeakers were separated by 90 degrees. Spatial configurations included both speech and (speech-weighted stationary) noise presented from the front (SONO), speech presented from the front and noise from the $\mathrm{Cl}$ side $(\mathrm{SONCl})$, and noise presented from the front and speech presented from the $\mathrm{Cl}$ side (SCINO). Tested listening conditions included listening with the acoustic-hearing ear only (monaural), and with both the acoustic-hearing ear and $\mathrm{Cl}$ or $\mathrm{TI}$ (binaural). The noise signal was presented at a constant level of $65 \mathrm{~dB}$ SPL. The initial speech level was $55 \mathrm{~dB}$ SPL and was adjusted in steps of $2 \mathrm{~dB}$ according to the response of the patient. Two lists, consisting of ten sentences each, of the LIST test were presented for each tested condition. The Speech Reception Threshold (SRT) was calculated by averaging the signal-to-noise ratio over the second list that was presented, in order to obtain a $50 \%$ correct score [25].

\section{5. Ethical consideration}

This case study was approved by the Ethics Committee of Maastricht University/academic hospital Maastricht in accordance with the Declaration of Helsinki.

\section{6. Results}

During TI fine tuning procedure 48 conditions were tested for five minutes each in order to find the best stimulation patterns to suppress tinnitus. The four most effective patterns were tested for two full days each in order to select the optimal stimulus as regards tinnitus reduction and comfort. The optimal stimulation pattern was subsequently used to assess the long-term effect of simple electrical stimulation on tinnitus. The optimal pattern, preferred by patient, was a stimulation of electrode 9 with a rate of 2820 pps, which was sinusoidal amplitude modulated with a modulation depth of $24 \%$ and a modulation frequency of $441 \mathrm{~Hz}$. Pulses were cathodic first with a final maximum charge value of $3.32 \mathrm{nC}$ and a phase duration of $37 \mu \mathrm{s}$ (Fig.2). After one day of stimulation the perceived stimulus loudness, was 0.8 on a VASscale $0-10$.

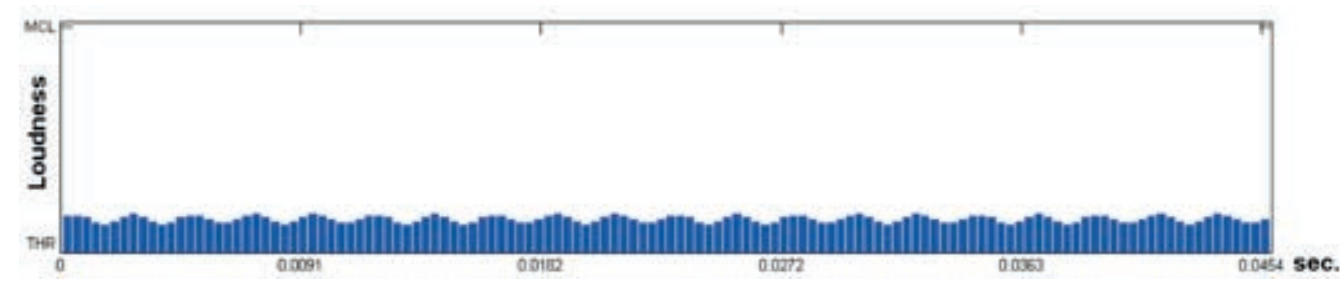

Figure 2: Optimal sinusoidal amplitude modulated stimulation pattern in TI mode. THR: Threshold (i.e. the level slightly below an audible stimulus), MCL: Most Comfortable Level (i.e. the highest level at which a stimulus is perceived as loud but not uncomfortable or painful). Loudness was set to $9 \%$ of the Dynamic Range, the range between THR and MCL. Inset: schematic presentation of a single biphasic stimulus which corresponds to a single bar in the presented stimulation pattern.

\section{6.1. Tinnitus}

Over time different post-operative tinnitus pitches ranging from narrow-band (1/3 octave) noise with a center frequency of $630 \mathrm{~Hz}$ to a narrow band with a center frequency of $6000 \mathrm{~Hz}$ were observed with a median of a narrow band noise with a center frequency of $2000 \mathrm{~Hz}$. Figure 3 shows tinnitus loudness in chronological order (see also Fig.1 for more detail). A robust reduction in tinnitus loudness over time is shown. However, tinnitus seemed to partly restore when stimulation was stopped for one week (Cl deact. and placebo).

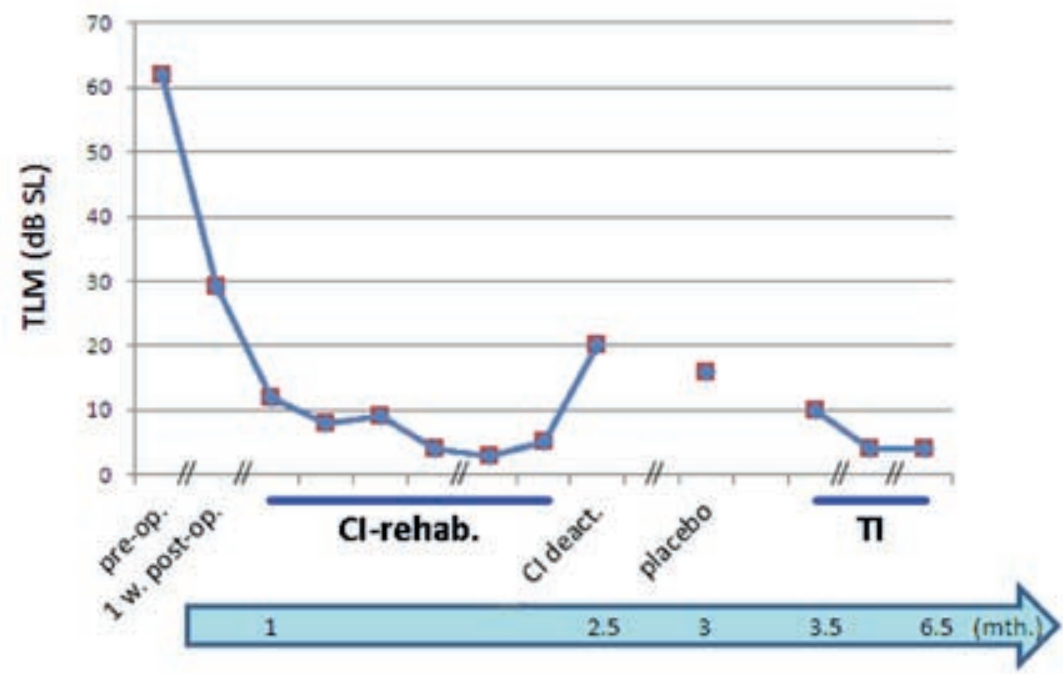

Figure 3: Results of tinnitus loudness matching.

The tinnitus loudness as well as the effect on life, extent of problems, and amount of discomfort due to the tinnitus were measured using the VAS method (Fig.4). A reduction in VAS scores over time is shown. Tinnitus-complaints seemed to come back when stimulation was stopped for one week (CI deact.). Tinnitus-complaints were also partly re-experienced after a week of sham stimulation as tinnitus was again suppressed during TI fine tuning procedure (data not shown).

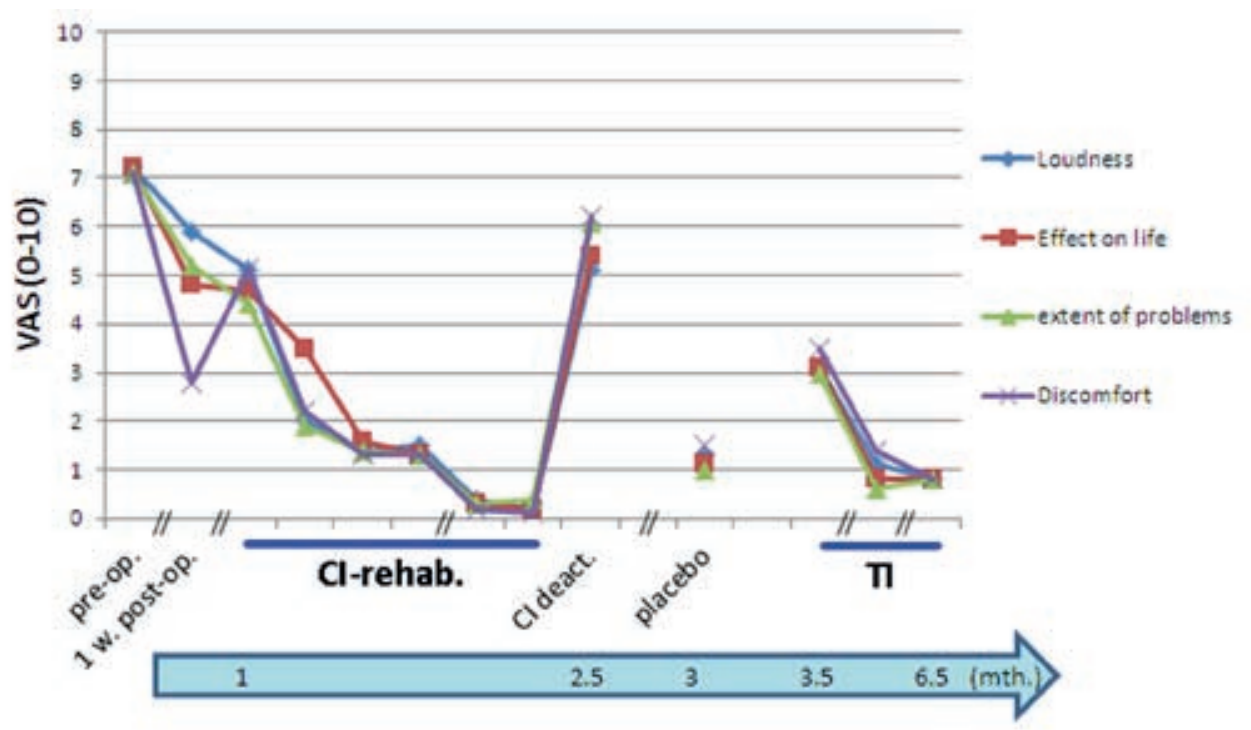

Figure 4: Results of VAS testing. The TI fine tuning procedure was performed between $\mathrm{Cl}$ deactivation and sham stimulation (see Fig.1) 
5. 6.2. Speech perception

During rehabilitation, the standard $\mathrm{Cl}$ was used (i.e. for perception of environmental sounds). For assessing speech discrimination in silence, the AH was shielded to prevent ceiling effects. Figure 5 shows speech discrimination tests at the third and eighth week of $\mathrm{Cl}$ rehabilitation and after three months of $\mathrm{TI}$ use. PTA of the contralateral normal hearing ear was stable over time. Shielding in the third week of rehabilitation seemed to mute the AH less compared to shielding in the eighth week. Nevertheless, speech discrimination with $\mathrm{Cl}$ in quiet improved over time, specifically at low speech levels. As the $\mathrm{TI}$ stimulated independently of the acoustic input, no improvement in speech discrimination with TI was expected. However, as Figure 5 shows, TI had no negative effect on speech discrimination in quiet either. Figure 5: Speech discrimination score in silence. Percentage phoneme score in a free-field-condition using

In addition, in order to shield the $\mathrm{AH}$ completely, monosyllable words were directly provided on the $\mathrm{Cl}$ via direct input. Here, a 70\% phoneme score was achieved in the eighth week of Cl rehabilitation (not shown in Fig.5).

Figure 6 shows speech perception in noise tested pre-operatively, in the eighth week of $\mathrm{Cl}$ rehabilitation and after three months TI-use Monaural means unilateral hearing with the normal hearing ear while binaural means bilateral "hearing" with additional $\mathrm{Cl}$ or TI in the deaf ear. In the eighth week of C rehabilitation, a significant improvement in speech perception from acoustic-hearing only (monaural) to acoustic-hearing together with $\mathrm{Cl}$ (binaural) is shown when speech is presented from the $\mathrm{Cl}$-side (SCINO) No differences between monaural and binaural hearing are shown in the other spatial conditions. Additionally, no differences in speech perception are shown between acoustic hearing only (monaural) and acoustic hearing together with $\mathrm{TI}$ (binaural) in any of the tested spatial configurations. Thus, no negative effects of TI on speech perception were observed.

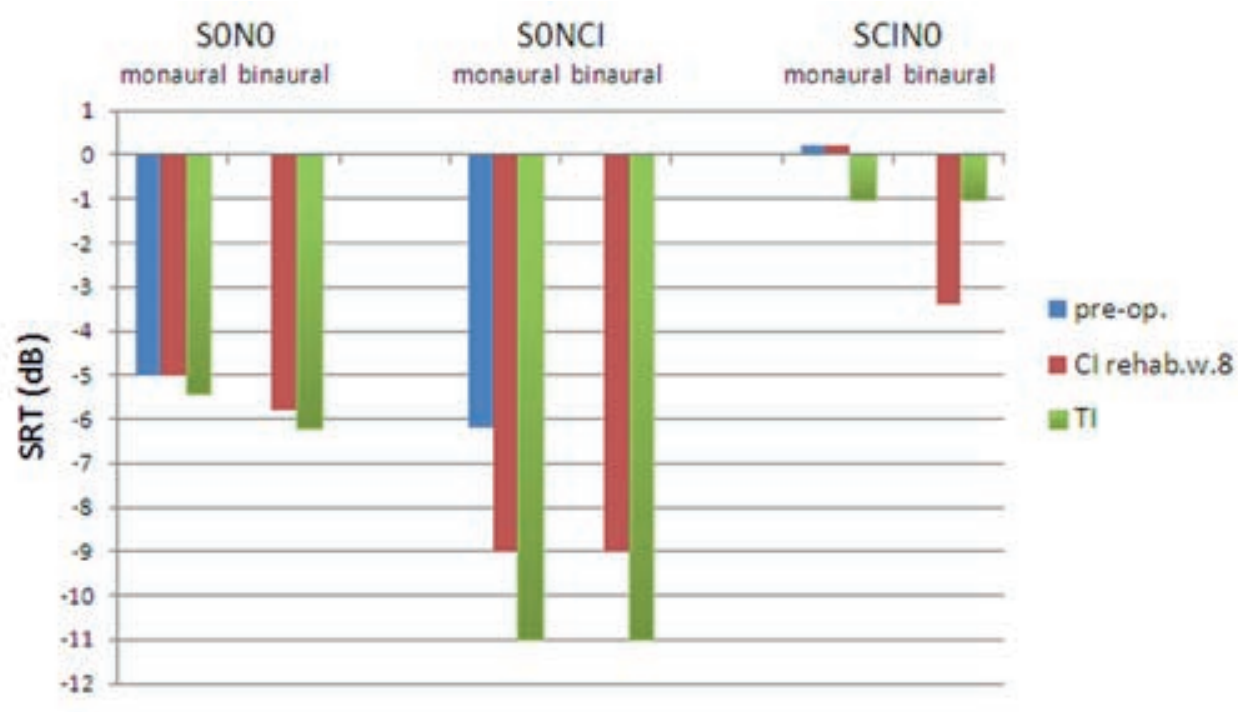

Figure 6: SRT scores for speech recognition in noise. SONO: speech and noise from front, SONCl: speech from front and noise from Cl-ear, SCINO: speech from Cl-ear and noise from front.

\section{Discussion}

In our subject, tinnitus was significantly reduced with intracochlear electrical stimulation. Stimulation was both dependent on $(\mathrm{CI})$ and independent of $(\mathrm{TI})$ the acoustic input. Stopping the stimulation for one week (Cl deact. and placebo) seems to be too short to completely reverse the effects of electric stimulation on tinnitus perception as the tinnitus is only partly restored (see Figs. 3 and 4). Results from TLM and VAS are generally consistent even though the effects of $\mathrm{Cl}$ deactivation ( $\mathrm{Cl}$ deact. and placebo) on tinnitus are different. During $\mathrm{Cl}$ deactivation ( $\mathrm{Cl}$ deact.), tinnitus restoration was more pronounced in VAS-scores compared totinnitusloudness in dBSL. For the placebocondition, tinnitus restoration wasless pronounced in VAS-scores as in tinnitus loudness in dB SL. This could probably be explained by the fact that the placebo condition was tested during subject's holiday allowing a greater acceptance of tinnitus which resulted in reduced VAS scores.

The optimal simple electrical stimulation found to suppress tinnitus was an AM sinusoidal stimulus on the pitch matched electrode (i.e. electrode which is, according to Greenwood's function [26], responsible for the tinnitus pitch matched via the contralateral ear). The pitch matched electrode is also the electrode which matched best the perceived tinnitus pitch by consecutively stimulating the single electrodes with a pulse rate of $2820 \mathrm{pps}$ in a 2-alternative forced choice method. A pulse rate of $2820 \mathrm{pps}$ was applied as it equals the tinnitus pitch (in $\mathrm{Hz}$ ), matched via the contralateral ear. This stimulation, offered on the pitch matched electrode is in accordance with Rubinstein and Tyler [21] who reported on an effective high rate pattern on the tinnitus pitch matched electrode. In contrast, Zeng and colleagues [7] reported on a low rate apical stimulation which significantly suppressed a 4000-8000 Hz tinnitus.

Additionally, successful tinnitus reducers from Zeng and colleagues [7] and Rothholtz and colleagues [27] seem to show a relation between stimulus location and stimulation rate. From this one could hypothesize that stimuli characteristics are optimal when stimulus rate equals corresponding frequency of the specific electrode location. This could explain why basal electrical stimulation (represents high frequencies) with 
low stimulation rate was ineffective [28]. However, these settings were often not tested in previous studies and have to be investigated in the future.

There is a significant decrease in speech discrimination in quiet when the $\mathrm{AH}$ was shielded and $\mathrm{Cl}$ was deactivated (shielded $\mathrm{AH}, \mathrm{no} \mathrm{Cl}$ ) in the eighth week compared to the third week of $\mathrm{Cl}$ rehabilitation (see Fig.5). As described above this could be due to a difference in attenuation. An additional explanation could be that our subject made in the eighth week much more use of his $\mathrm{Cl}$ than in the third week and does therefore not rely on the shielded acoustic input signal. Speech discrimination in quiet with Cl only (shielded AH, Cl) was, for the lower loudness intensities (55 and $65 \mathrm{~dB} \mathrm{SPL}$ ), improved in the eighth week compared to the third week of CI rehabilitation (Fig.5). No improvement could be observed for the higher loudness intensity ( $75 \mathrm{~dB} \mathrm{SPL}$ ) which is probably due to the limited shielding of the earplug and earmuff. Speech discrimination in quiet with $\mathrm{Cl}$ only and via direct input in the eighth week of $\mathrm{Cl}$ rehabilitation is analogous to a report on four SSD subjects after six months of Cl use [29]. A report on an SSD child implanted with a $\mathrm{Cl}$ shows worse performance with $\mathrm{Cl}$ only after two months of $\mathrm{Cl}$ use, but gradually improved during at least six months of $\mathrm{Cl}$ use [30]. Because improving hearing was not our primary objective, $\mathrm{Cl}$ rehabilitation was intermittent partially incomplete when stable loudness perception on electrical stimulation was achieved.

In the eighth week of $\mathrm{Cl}$ rehabilitation, binaural speech perception in noise improved compared to monaural (AH only) speech perception in noise when speech was presented from the $\mathrm{Cl}$ side and noise was presented from front. These results are consistent with a previous report showing a significant improvement of binaural spatial hearing compared to monaural hearing (AH only) in the SCINO condition but no significant improvement in the SONO and SONCI condition [25]. Improvement of speech perception was expected in binaural hearing compared to monaural hearing in the SCINO condition. This improvement is due to head shadow effect and squelch effect. Furthermore, no negative effect of $\mathrm{TI}$ on speech perception in noise was observed.

The improvement in speech perception in the eighth week of $\mathrm{Cl}$ rehabilitation (both monaural and binaural) in the SONCI condition and after three months of TI-use (both monaural and binaural) compared to pre-operative performance is striking. A possible explanation could be that the reduced tinnitus loudness improves speech perception [31]. This positive effect was maintained after TI deactivation (monaural). Probably due to Residual Inhibition (RI) which was more than 30 minutes.

Both standard $\mathrm{Cl}$ as $\mathrm{Tl}$ show robust tinnitus suppression. This trial shows that environmental sound perception is not required and tinnitus can also be suppressed by a less sophisticated pulse generator. Therefore, TI provides a possible treatment option in case $\mathrm{Cl}$ is not appropriate. On the other hand, TI could probably (when choosing the optimal stimulus) optimize tinnitus suppression when a sub-optimal effect was achieved with standard Cl.

In conclusion, the present study reports on long-term reduction of tinnitus with simple intracochlear electrical stimulation in a SSD individual with unilateral severe tinnitus. No negative, but even a positive effect of $\mathrm{TI}$ on speech perception in noise could be observed. Furthermore, the additional use of a clinical $\mathrm{Cl}$ is advantageous for spatial speech discrimination in noise in our SSD subject. Because this report describes a single case, further research on long-term effects of simple electrical stimulation to suppress tinnitus is highly recommended.

\section{Acknowledgements}

We thank our subject for his participation in the present study and two anonymous reviewers for their comments on an earlier draft of the manuscript. Furthermore, the authors were very grateful to $d r$. Katrien Vermeire for the effort during protocol development. This work was supported by a research grant from MED-EL Corporation, Innsbruck, Austria. The funding source had no involvement in the study design; in the collection, analysis and interpretation of data; in the writing of the report; or in the decision to submit the article for publication. Informed consent was obtained from our subject. 
8. References

1. Langguth B, Kreuzer PM, Kleinjung T, De Ridder D. Tinnitus: causes and clinical management. Lancet Neurol 2013; 12: 920-30.

2. Nondahl DM, Cruickshanks KJ, Huang G-H, et al. Generational differences in the reporting of tinnitus. Ear Hear 2012; 33: 640-644.

3. Gilles A, Van Hal G, De Ridder D, Wouters K, Van de Heyning P. Epidemiology of noise-induced tinnitus and the attitudes and beliefs towards noise and hearing protection in adolescents.

PLOS ONE 2013; 8(7): e70297.

Hoffman HJ, Reed GW. Epidemiology of tinnitus. In: Snow JB, ed. Tinnitus: theory and management. London, BC Decker, 2004; 16-41.

5. Baguley D, McFerran D, Hall D. Tinnitus. Lancet 2013; 382: 1600-07.

6. Knipper M, Van Dijk P, Nunes I, Rüttiger L, Zimmermann U. Advances in the neurobiology of hearing disorders: recent developments regarding the basis of tinnitus and hyperacusis. Pros. Neurobiol 2013; 111: 17-33 DOI: 10.1016/J.pneurobio.2013.08.002.

7. Zeng F-G, Tang Q, Dimitrijevic A, Starr A, Larky J, Blevins NH. Tinnitus suppression by low-rate electric stimulation and its electrophysiological mechanisms. Hear Res 2011; 277: 61-66.

8. Eggermont JJ, Roberts LE. The neuroscience of tinnitus. Trends Neurosci 2004; 27:676-82.

9. Schaette R, McAlpine D. Tinnitus with a normal audiogram: physiological evidence for hidden hearing loss and computational model. The journal of neuroscience 2011; 31 (38): 1345213457.

10. De Ridder D, Elgoyhen AB, Romo R, Langguth B. Phantom percepts: tinnitus and pain as persisting aversive memory networks. Proc Natl Acad Sci USA 2011:108: 8075-80.

11. Billock VA, Tsou BH. Elementary visual hallucinations and their relationships to neural pattern-forming mechanisms. Psychol Bull 2012; 138 (4): 744-74.

12. Naycheva L, Schatz A, Röck T, et al. Phosphene threshold elicited by transcorneal electrical stimulation in healthy subjects and patients with retinal diseases. IOVS 2012; Vol.53 No.12: 7440-8.

13. Heller MF, Bergman M. Tinnitus aurium in normally hearing persons. Ann Otol 1953; 62: 73-83.

14. Kiani F, Yoganantha U, Tan CM, Meddis R, Schaette R. Off-frequency listening in subjects with chronic tinnitus. Hear Res 2013; 306: 1-10.

15. Bishop CE, Ashford A, Galster J, Windmill I. Hearing aid use by unilaterally impaired individuals : outcome of the tinnitus handicap inventory. Otolaryngology 2012; 2: 122. DOI: 10.4172/2161-119x.1000122.

16. Kim D-K, Bae S-C, Park K-H, et al. Tinnitus in patients with profound hearing loss and th effect of cochlear implantation. Eur Arch Otorhinolaryngol 2013; 270: 1803-1808

17. Van de Heyning P, Vermeire K, Diebl M, Nopp P, Anderson I, De Ridder D. Incapacitating unilateral tinnitus in single-sided deafness treated by cochlear implantation. Ann Oto Rhinol Laryn 2008; 117 (9): 645-652.

18. Arts RA, George EL, Stokroos RJ, Vermeire K. Review: Cochlear implants as a treatment of tinnitus in single-sided deafness. Curr Opin Otolaryngol Head Neck Surg 2012; 20: 398-403. Vlastarakos PV, Nazos K, Tavoulari E-F, Nikolopoulos TP. Cochlear implantation for single-sided deafness: the outcomes. An evidence-based approach. Eur Arch Otorhinolaryngol 2013; [Epub ahead of print].
Fretz RJ, Fravel RP. Design and function: a physical and electrical description of the 3M House Cochlear Implant system. Ear Hear 1985; 6: 14-19.

21. Rubinstein JT, Tyler RS, Johnson A, Brown CJ. Electrical suppression of tinnitus with high-rate pulse trains. Otol Neurotol 2003; 24: 478-485.

22. Chang JE, Zeng F-G. Tinnitus suppression by electric stimulation of the auditory nerve. Frontiers in systems neuroscience 2012; vol.6 art.19: 1-11.

23. Buechner A, Brendel M, Lesinski-Schiedat A, et al. Cochlear implantation in unilateral deaf subjects associated with ipsilateral tinnitus. Otol Neurotol 2010;31: 1381-1385.

24. Van Wieringen A, Wouters J LIST en LINT: sentences and numbers for quantitying speech understanding in severely impaired listeners for Flanders and The Netherlands. Int J Audiol 2008; 47: 348-355

25. Vermeire K, Van de Heyning P. Binaural hearing after cochlear implantation in subjects with unilateral sensorineural deafness and tinnitus. Audiol Neurotol 2009; 14: 163-171.

26. Greenwood DD. A cochlear frequency-position function for several species-29 years later. $\mathrm{J}$ Acoust. Soc. Am. 1990; 87: 2592-2605.

27. Rothholtz VS, Tang Q, Wu EC, Fine EL, Djalilian H, Zang F-G. Exploring the parametric space of tinnitus suppression in a patient with a cochlear implant. Laryngoscope 2009; 119: s177-s177.

28. Kleine Punte A, De Ridder D, Van de Heyning P. On the necessity of full length electrical cochlear stimulation to suppress severe tinnitus in single-sided deafness. Hear Res 2012; 295 24-9.

29. Stelzig $Y$, Jacob R, Mueller J. Preliminary speech recognition results after cochlear implantation in patients with unilateral hearing loss: a case series. Journal of Medical Case Reports 2011; 343.

30. Plontke SK, Heider C, Koesling S, et al. Cochlear implantation in a child with posttraumatic single-sided deafness. Eur Arch Otorhinolaryngol 2013; 270 (5): 1757-61.

31. Mertens G, Kleine Punte A, De Ridder D, Van de Heyning P. Tinnitus in a single-sided deaf ear reduces speech reception in the nontinnitus ear. Otol Neurotol 2013; 34: 662-666. 


\section{Chapter}

Tinnitus suppression by intracochlear electrical stimulation in Single Sided Deafness a prospective clinical trial: Part I

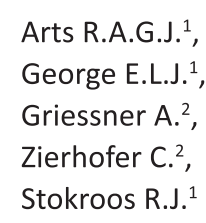

Zierhofer $\mathrm{C}^{2}$

Stokroos R.J.

1Department of ENT/Head and Neck Surgery,

Maastricht University Medical Centre,

PO Box 5800, 6202 AZ Maastricht, The Netherlands

2Institute of Mechatronics, University of Innsbruck Technikerstraße 13, 6020 Innsbruck, Austria

Accepted: Ear Nose Throat J

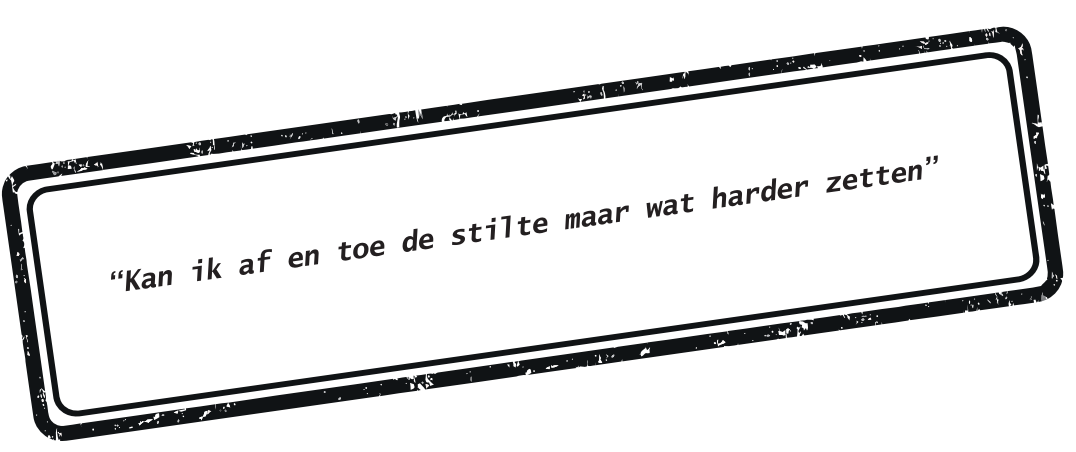


Abstract:

Cochlear implantation is a viable treatment option for tinnitus, but the underlying mechanism is yet unclear. Is the tinnitus suppression due to the reversal of the assumed maladaptive neuroplasticity or is it the shift in attention from the tinnitus to environmental sounds and therefore a reduced awareness that reduces tinnitus perception? In this prospective trial, ten patients with Single Sided Deafness were fitted with a cochlear implant to investigate the effect of looped intracochlear electrical stimulation (i.e. stimulation that does not encode environmental sounds) on tinnitus, in an effort to find optimal stimulation parameters. Variables under investigation were: amplitude (perceived stimulus loudness), anatomical location inside the cochlea (electrode/electrodes), amplitude modulation, polarity (cathodic/ anodic first biphasic stimulation) and stimulation rate. The results suggest that tinnitus can be reduced with looped electrical stimulation, in some cases even with inaudible stimuli. The optimal stimuli for tinnitus suppression appear to be subject-specific. However, medium-to-loud stimuli suppress tinnitus significantly better than soft stimuli, which partly can be explained by the masking effect. Although the long-term effects on tinnitus would still have to be investigated and will be described in Partll, intracochlear electrical stimulation seems a potential treatment option for tinnitus in this population.

\section{Introduction:}

Subjective tinnitus is an auditory phantom sensation that is imperceptible by someone other than the sufferer. The estimated prevalence in the general population is about 10\%-15\% [Langguth et al., 2013] and is expected to increase in the future [Nondahl et al., 2012; Gilles et al., 2013]. Tinnitus can be extremely bothersome which may lead to depression and serious problems in daily life [Turner et al., 2007]. To date, tinnitus remains refractory to current medical treatment and therefore only management treatments and therapies can be offered [Jastreboff 1999; Cima et al., 2012]

While the exact cause of tinnitus remains unknown, several mechanisms underlying tinnitus are suggested. Some of these are changes in central gain to compensate for hearing loss, increased spontaneous activity and synchrony of cortical neurons by tonotopic reorganization and a shift in the balance of excitatory and inhibitory neurotransmitters [Eggermont and Roberts, 2012; Roberts et al., 2013]. The current consensus is that tinnitus is the result of maladaptive plasticity in the central auditory pathway as a result of auditory deprivation [Engineer et al., 2011]. This is supported by the results of a number of studies: tinnitus development after simulating unilateral hearing loss [Schaette et al., 2012], a tinnitus prevalence of $75-90 \%$ in otosclerotic patients [Schaette, 2014] and $80 \%$ in the idiopathic sudden sensorineural hearing loss population [Nosrati-Zarenoe et al., 2007], a tinnitus incidence of 58\% after cutting the cochlear nerve for removal of acoustic tumors [Berliner et al., 1992] and the ineffectiveness of cochlear nerve section as a treatment option for tinnitus [House and Brackmann, 1981]. Furthermore, in $85-90 \%$ of tinnitus cases hearing loss is detected [Sanchez and Ferrari, 2002] and even "hidden hearing loss" could trigger tinnitus [Schaette and McAlpine, 2011; Modh et al., 2014].

Based on unwanted neural plasticity as the underlying cause, tinnitus should be reversible by increasing auditory stimulation, known as the 'bottom-up approach'. Acoustic sound enrichment by either conventional hearing aids [Saltzman and Ersner, 1947; Shekhawat et al., 2013; Hoare et al., 2014], tinnitus maskers [Erlandsson et al. 1987; Vernon and Meikle, 2003; Fioretti et al., 2012] or music with tinnitusrelated modification [Davis et al., 2007; Tass et al., 2012; Pantev et al., 2012a; Pantev et al., 2012b; Reavis et al., 2012] is a potential treatment option but shows a large variability in effectiveness among patients. This variability may be explained by the heterogeneity of tinnitus, which is caused by auditory deprivation and can occur at various positions along the auditory pathway. This heterogeneity is substantial and has hampered both basic science and treatment research [Baguley et al., 2013]. As the most common cause of auditory deprivation is in the cochlea at the level of the hair cells, treatment options need to bypass this pathology.

Increasing evidence seems to indicate that electrical sound enrichment may be a promising alternative for its acoustic counterpart. Electrical stimulation bypasses the cochlear transduction of acoustic signals and instead directly stimulates the cochlear nerve. In the early 19th century, Alessandro Volta was the first person who ever electrically stimulated the hearing system in humans [Wilson and Dorman, 2008] Almost two centuries later, House [1976] reported tinnitus suppression in one of the first cochlear implant $(\mathrm{Cl})$ patients. Although the primary goal of cochlear implantation is to provide a sense of sound to a person who suffers from severe to profound hearing loss, during decades of research it has become clear that tinnitus suppression is a common side effect [Ito and Sakakihara, 1994; Aschendorff et al., 1998; House, 1999; Steenerson and Cronin, 1999; Ruckenstein et al., 2001; Miyamoto and Bichey, 2003; Di Nardo et al., 2007; Tyler et al., 2008; Olze et al., 2011; Olze et al., 2012; De Carvalho et al., 2013]. Nevertheless, tinnitus can also be a complication after cochlear implantation [Di Nardo et al., 2007; 


\section{Materials and Methods:} et al., 2014; Todt et al., 2014].

Van de Heyning et al. [2008] reported the first results of cochlear implantation in an attempt to treat tinnitus in single-sided deafness (SSD), after which many studies followed on tinnitus suppression in SSD [Kleinjung et al., 2009; Arndt et al., 2010; Buechner et al., 2010; Palau et al., 2010; Jacob et al., 2011; Kleine Punte et al., 2011; Firszt et al., 2012b; Mendes et al., 2012; Ramos et al., 2012; Hansen et al., 2013; Távora-Vieira et al., 2013, 2015 and reviewed by Arts et al., 2012; Blasco and Redleaf, 2014; Tokita et al., 2014; Vlastarakos et al., 2014; van Zon et al.,2015]. As a common conclusion it can be stated that cochlear implantation is a viable treatment option for tinnitus in SSD.

The question arises what mechanism is responsible for tinnitus suppression after cochlear implantation. Is this suppression due to the reversal of the assumed maladaptive plasticity or is it simply the shift in attention from the tinnitus to environmental sound and therefore less awareness that reduces tinnitus perception [Searchfield et al., 2012]? The former mechanism is supported by the presence of residual inhibition in many patients, a persistency in the tinnitus reduction after the stimulation is deactivated [Osaki et al., 2005; Arts et al., 2015]. Therefore, it should be possible to treat tinnitus by intracochlea electrical stimulation that does not directly encode acoustic sound. This intracochlear electrical stimulation, independent of external acoustic signals, forms a potential new treatment option for tinnitus.

Prior studies show short-term tinnitus suppression of intracochlear electrical stimulation independent of external acoustic sounds on tinnitus [Rubinstein et al., 2003; Zeng et al.,2011; Chang and Zeng, 2012; Arts et al., 2015]. Optimal stimulus characteristics seem to be subject-specific, which could be explained by the heterogeneity of the underlying tinnitus pathophysiology and perception.

In order to assess the potential clinical benefit of intracochlear electrical stimulation without an acoustic input signal on tinnitus suppression, studies on the long-term effects are needed. However, only two long-term case reports (of which one anecdotally) on improvement of tinnitus during stimulation are available [Vernon and Meikle, 2003; Arts et al., in press]. The current study therefore aims to furthe examine these long-term effects, applying a subject-specific adjustment of stimulation characteristics fo optimal tinnitus suppression. The study design is in two parts: in Part I we aim to find potentially optimal characteristics for tinnitus suppression while in Part II the long-term effects of the found stimuli will be investigated. Furthermore, in Part I we aim to answer three additional research questions: (1) Does the surgery itself suppress tinnitus? (2) Does the clinical Cl suppress tinnitus? (3) Is a 1-week period of C deactivation sufficient to rule out the possible influence of providing a hearing sensation on the experience of tinnitus? Here, we reported on the results of Part I, while the results of the (ongoing) Part I will be reported elsewhere.

\section{Subjects}

Ten adult SSD subjects were included with an audiometric hearing loss of at least $70 \mathrm{~dB}$ pure-tone average, averaged across $0.5,1$ and $2 \mathrm{kHz}$ in one ear. Their contralateral ear had a moderate to normal audiometric hearing threshold (pure-tone average better than $50 \mathrm{~dB} \mathrm{HL}$ ); in case of moderate hearing, a hearing aid should at least have been tried and have had no positive effect on tinnitus complaints. The contralateral ear with moderate to normal audiometric hearing threshold was required to enable speech perception during Part II where the long-term effects of intracochlear electrical stimulation independent of external acoustic sounds on tinnitus will be investigated. In addition, this contralateral functional hearing allows us to perform psychoacoustic measurements of tinnitus characteristics which is essentia in the current part of the study. Here, pitch matching their tinnitus via the contralateral ear was performed in order to customize the looped stimulation patterns based on the individual tinnitus characteristics. The subjects suffered from unilateral, chronic (at least 2 years), continuous and severe tinnitus perceived at the hearing-impaired ear (table 1). The perceived tinnitus was required to be stable for at least 1 year, giving rise to at least moderate to severe complaints, with a Tinnitus Handicap Inventory (THI) [Newman et al., 1996; Zeman et al., 2012] score of at least 38 and/or a Tinnitus Questionnaire (TQ) [Hallam et al., 1988: Meeus et al 2007: Zeman etal. 2012] score of at least 42. Both questionnaires were administered before implantation. Furthermore, the tinnitus loudness was at least 7.0 on a visual analogue scale (VAS) with a range from 0 to 10

Main exclusion criteria were: pulsatile tinnitus, congenital malformation of the auditory system, history of vestibular schwannoma, active middle ear disease and ossification of the cochlea. Furthermore, patients with a history of psychiatric disorders, depression and use of antidepressant medication were excluded for participation. In order to exclude underlying depression disorders, the Beck Depression Inventory (BDI) was used. The BDI is a 21-item questionnaire that explicitly stresses the aspects of depression, where each item can be scored from 0 to 3 [Beck et al., 1961; Beck and Beamesderfer, 1974; Bouman et al., 1985]. The total score should not exceed 19 points (minimal or light depression).

The average age at time of surgery was 48.1 years (range: $31.7-67.9$ years) with an average tinnitus duration of 10.5 years (range: 2-27 years). The mean duration of deafness was 11.3 years (range: 2-27 years) with varying etiology (table 1 ).

All SSD subjects were counseled before implantation concerning the risk and possible negative outcomes of cochlear implantation, explaining that no guarantees for tinnitus suppression could be given. This study was approved by the Ethics Committee of Maastricht University/Academic Hospital Maastricht and was performed in accordance with the declaration of Helsinki (approval No. NL38789.068.11). All subjects gave written informed consent before participation. 
Four to six weeks after surgery standard clinical $\mathrm{Cl}$ fitting and rehabilitation were started. During this clinically standardized procedure subjects got used to electrical stimulation of the auditory pathway in order to provide awareness of environmental sounds and speech understanding. The dynamic range was optimized and stabilized by an experienced $\mathrm{Cl}$ audiologist. This 'standard $\mathrm{Cl}$ rehabilitation' procedure took about 2 months.

After rehabilitation, the $\mathrm{Cl}$ was deactivated for 1 week to rule out the possible influence of providing a hearing sensation on the experience of tinnitus. Then, electrical stimulation independent of external acoustic signals was applied via the reprogrammed $\mathrm{Cl}$, from now called 'tinnitus implant' (TI). Durin the 'fine-tuning procedure', a large number of different stimulation patterns were repeatedly presented via the $\mathrm{TI}$ in a looped way with a total stimulus duration of $5 \mathrm{~min}$ for each pattern. The goal of this procedure was to find the patterns which provide the optimal combination of tinnitus suppression and stimulation comfort for each subject.

Subsequently, the four most suitable stimulation patterns in each subject were tested for 2 days each in order to select the stimulation pattern optimal for daily use. These results and the longitudinal effects during our crossover design (part II) will be reported in a later stage.

\section{Device and Software}

Subjects were implanted with the MED-EL cochlear implant system (MED-EL Corp., Innsbruck, Austria), consisting of the CONCERTO implant, the OPUS2 speech processor, and fitted with the standard clinical software, Maestro version 4.1.2. The FS4 processing strategy was applied in all subjects. For complete insertion, all 12 electrodes were positioned inside the cochlea with electrode 1 in the apical region and electrode 12 in the basal region of the cochlea. During the fine-tuning procedure the OPUS2-processor was programmed to function as $\mathrm{TI}$, a pattern generator which provided intracochlear electrical stimulation with looped patterns using the 128-byte random-access memory integrated in the OPUS2, and was programmed using customized software (Institute of Mechatronics, Innsbruck, Austria) in Matlab version 7.11.0 (R2012b) (The Mathworks, Natick, Mass., USA). Both Cl and TI adjustments were performed using MED-EL's Diagnostic Interface Box II.

\section{Fine-Tuning $\mathrm{T}$}

During the fine-tuning procedure the TI was used to continuously generate charge-balanced, biphasic pulses in monopolar mode. In order to find the optimal stimulation characteristics for tinnitus suppression, 48 stimulus conditions were tested for $5 \mathrm{~min}$ each. Variables under investigation were: amplitude (perceived stimulus loudness; the VAS was used for adjusting the stimulus level until it was rated 2, 5 or 7 depending on the tested condition), anatomical location inside the cochlea (electrode/ electrodes), amplitude modulation (see fig. 1 for details), polarity (cathodic/anodic first) and stimulation rate (for an overview and details see table 2 and Appendix 1). In case electrodes could not be used for medical reasons, these electrodes were switched off in this study including the fine-tuning procedure. Patterns were tested in random order. The number of test days per subject depended on the duration of residual inhibition and participant's fatigue. 
Table 2: An overview of the 48 tested looped patterns. See Appendix 1 for pattern numbers. Conditions that were technically not feasible because of a limit to the overall (sum of each activated electrode) stimulation rate were excluded. Top half: overview of 32 conditions (any combination of settings was tested). Bottom half: 16 additional conditions. For the extra conditions, the presented settings were combined with the default settings ( $)$ of the 2 default settings were used for the stimulation rate PM elec $=$ Tinnitus pitch-matched electrode.

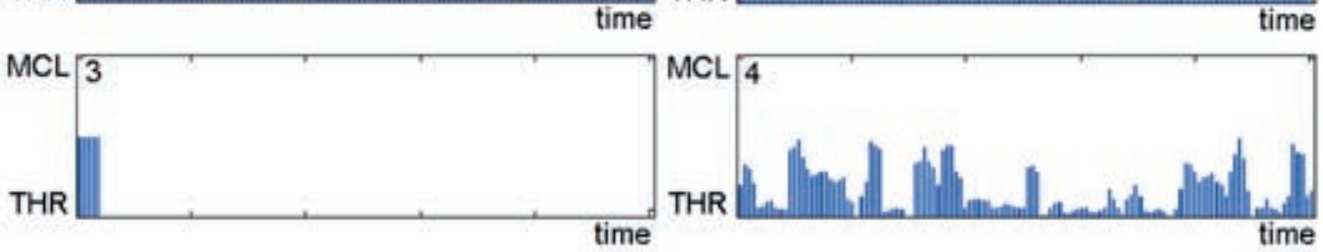

Fig.1: Tested amplitude modulations. Each loop consisted of 128 pulses, with a total duration depending on the used stimulation rate. MCL: Maximum Comfortable Level, THR: Threshold level 1: Fixed (no modulation), 2: Sine wave [20 sine waves per loop, modulation depth is (30/128)*100\%], 3: Burst (per loop, trains of five consecutive pulses followed by a gap with a duration depending on the used stimulation rate) 4 : Random (modulation depth is $100 \%)$

The electrode that could elicit a pitch perceived closest to tinnitus pitch (the pitch-matched electrode) was determined by pitch matching via acoustic stimulation of the contralateral ear (for details see section 'Outcome Measurements'). The default frequency tables in the standard clinical Maestro software were used to translate the (center) frequency of the tinnitus to the corresponding electrode.

Determination of the tinnitus pitch-matched electrode via stimulation of the implanted electrodes was also performed. Here, a two-alternative forced-choice method was used [Arts et al., 2015]. Electric stimuli consisted of trains of biphasic pulses presented at rates (in pulses per second, pps) equal to the tinnitus pitch (in Hertz) previously matched via the contralateral ear.

However, tinnitus pitch matching via stimulation of the implanted electrodes regularly turned out to be impossible since subjects were either not able to choose between perceptions or the subjects indicated that there was a large discrepancy between the obtained pitch and their tinnitus perception. Moreover, tinnitus pitch matching via stimulation of the implanted electrodes seems suboptimal as besides stimulation place the pitch perception is also affected by stimulation polarity, stimulation rate and stimulation level [Carlyon at al., 2013; Schatzer et al., 2014; Arts et al., 2015]. Therefore, different stimulation characteristics than used in this matching procedure may result in different tinnitus pitchmatched electrodes.

For the subjects where both matching procedures were completed, a high degree of consistency was found (data not shown). Therefore and in order to keep the procedure for the determination of the tinnitus pitch-matched electrode constant between subjects, the electrode determined via acoustic stimulation of the contralateral ear was the preferred method.

\begin{tabular}{|c|c|c|}
\hline Variables & Setting & Default \\
\hline \multirow[t]{2}{*}{ Amplitude } & Loudness on a VAS(0-10) $=5$ & * \\
\hline & Loudness on a VAS(0-10) $=2$ & \\
\hline \multirow[t]{2}{*}{ Location } & Electrode responsible for the perceived tinnitus frequency (PM elec.) & * \\
\hline & All electrodes & \\
\hline \multirow[t]{2}{*}{ Modulation } & Random & * \\
\hline & Fixed (no modulation) & \\
\hline Polarity & Biphasic: cathodic first & * \\
\hline \multirow[t]{4}{*}{ Rate (pps/channel) } & Pitch matched (equal to the frequency of the perceived tinnitus) & * \\
\hline & 5000 & * \\
\hline & 750 & \\
\hline & 200 & \\
\hline Total: & $2 * 2 * 2 * 1 * 4=32$ looped patterns & \\
\hline Variables & Setting & $\begin{array}{l}\text { Extra } \\
\text { conditions }\end{array}$ \\
\hline Amplitude & Loudness on a VAS(0-10) $=7$ & 2 \\
\hline \multirow[t]{4}{*}{ Location } & $\begin{array}{l}\text { Electrode responsible for the perceived tinnitus frequency } \\
\text { (PM elec.) and two adjacent electrodes }\end{array}$ & 2 \\
\hline & $\begin{array}{l}\text { Two electrodes adjacent to (but without) electrode responsible } \\
\text { for the perceived tinnitus frequency (PM elec.) }\end{array}$ & 2 \\
\hline & Most basal located electrode & 2 \\
\hline & Most apical located electrode & 2 \\
\hline \multirow[t]{2}{*}{ Modulation } & Burst & 2 \\
\hline & Sine wave & 2 \\
\hline Polarity & Biphasic: anodic first & 2 \\
\hline Total: & & 16 \\
\hline
\end{tabular}

\section{Outcome Measurements}

\section{General}

In order to assess the tinnitus distress during the experiments, we have used a VAS method, psychoacoustic measurements (pitch and loudness matching) and questionnaires (THI and TQ). For the VAS method, the subjects marked how they perceived tinnitus loudness, amount of discomfort, effect on life and extent of problems due to the tinnitus, each on a $10-\mathrm{cm}$ bar. At the left of this bar ' 0 no tinnitus', '0 no discomfort', 
'0 no effect on life' or '0 no problems' and at the right' 10 extremely loud', '10 extremely burdensome','10 maximal effect on life' or' 10 extremely problematic' were indicated, respectively [Buechner et al., 2010; Arts et al., in press]. Values were accurate to one decimal place. VAS measurements were performed prior to the surgery, both before after standard clinical $\mathrm{Cl}$ rehabilitation and after 1 week of $\mathrm{Cl}$ deactivation.

Tinnitus pitch and loudness were measured, under headphones (Telephonics, TDH-39P), via the contralateral acoustic hearing ear. Tinnitus pitch matching was performed, prior to the surgery, both before and after standard $\mathrm{Cl}$ rehabilitation, after 1 week of $\mathrm{Cl}$ deactivation and at the start of each test day during the fine-tuning procedure. A three-alternative forced-choice method was used. Pure tones, 1/3-octave narrowband noises and warble tones within the $250-$ to $8,000-\mathrm{Hz}$ range (for the center frequency in case of narrowband noises) and white noise were presented. Both octave and interoctave frequencies were presented in separate 'runs' from low to high frequency and from high to low frequency respectively [Arts et al. 2015]. Subjects were asked to concentrate on the predominant pitch of their tinnitus.

Acoustic tinnitus loudness matching was measured in decibel sensation level (dBSL) using predetermined tinnitus pitch. Subjects were asked to indicate whether their tinnitus was louder or softer than the stimulus. A two-down one-up adaptive staircase rule [Leek, 2001] with step sizes of $5 \mathrm{~dB}$ for rough determination and $1 \mathrm{~dB}$ for precise determination were used. The starting point was at $15 \mathrm{~dB} \mathrm{SL}$. Tinnitus loudness matching was performed, prior to the surgery, both before and after standard $\mathrm{Cl}$ rehabilitation, after 1 week of $\mathrm{Cl}$ deactivation and at the start of each test day during the fine-tuning procedure.

The degree of handicap due to the perceived tinnitus was evaluated with the THI prior to the surgery and both before and after $\mathrm{Cl}$ rehabilitation. The THI, which has been internationally validated, quantifies the effect of tinnitus on the patient's emotions and daily activities. Subjects were asked to answer the 25 items of the self-administered questionnaire with 'no' (0 points), 'sometimes' (2 points) or 'yes' (4 points), with a higher THI score indicating a more severe handicap [Newman et al., 1996; Zeman et al., 2012]

Distress caused by the tinnitus was evaluated with the Dutch validated TQ [Meeus et al., 2007] prior to the surgery and both before and after $\mathrm{Cl}$ rehabilitation. The TQ consists of 52 questions, with a maximum score of 84 . Again, a higher score indicates a more severe distress.

\section{Fine-Tuning Procedure}

Before and every $30 \mathrm{~s}$ during the $5 \mathrm{~min}$ of each stimulation, subjects had to rank the perceived tinnitus and stimulus loudness at that specific moment on the VAS. Subjects indicated loudness perception by setting a mark on a $10-\mathrm{cm}$ bar, on which at the left ' 0 no tinnitus' or ' 0 inaudible' and at the right '10 extremely loud' was indicated [Kleine Punte et al., 2013; Arts et al., 2015]. In order to bring the tinnitus back to its baseline loudness level before testing the next condition, a maximum recovery time of $30 \mathrm{~min}$ was allowed. The amount of tinnitus reduction due to electrical stimulation was expressed in percent relative to the baseline loudness and can be calculated using the following equation [Arts et al., 2015]:

$$
R_{t}(\%)=\frac{L_{0}-L_{t}}{L_{0}} \times 100 \%
$$

Here, $R_{t}$ represents the amount of tinnitus reduction and $L_{0}$ represents the perceived tinnitus loudnes at baseline, that is, in absence of and before stimulation (TI switched off). The most extreme loudness (the biggest change, positive or negative, relative to $L_{0}$ ), ranked on the VAS during stimulation is denoted as $L$ A tinnitus reduction of $0 \%$ corresponds with no change in perceived tinnitus loudness while positive values correspond to tinnitus reduction and negative values correspond to a tinnitus aggravation. Tinnitus loudness changes of $30 \%$ or more were considered as clinically relevant and a reduction of $\geq 30 \%$ was considered as successful [Reavis et al., 2010; Chang et al., 2012; Arts et al., 2015].

\section{Statistics}

\section{Standard Cl Rehabilitation}

Using this study design, three different research questions that were specified a priori were answered: (1) Does the surgery itself suppress tinnitus? (2) Does the clinical Cl suppress tinnitus? (3) Is a 1-week period of $\mathrm{Cl}$-deactivation sufficient to rule out the possible influence of providing a hearing sensation on the experience of tinnitus? In order to answer these research questions, nonparametric one-sided Wilcoxon signed-rank tests for paired measurements were performed. Individual results and median were presented. Statistics were performed with IBM SPSS Statistics, version 20, and p values smaller than 0.05 were considered statistically significant.

\section{Fine-Tuning Procedure}

In order to find the optimal parameters for tinnitus suppression exploratory analyses were performed using the within-subject mean effect sizes of the stimulation patterns within the condition of interest. The within-subject mean effect size was calculated as the pooled mean difference per person between the paired measurements in which all the variables, except the variable under investigation, were kept constant ( within, but not between the paired measurements). Paired measurements containing missing values were excluded from analyses. Nonparametric Friedman tests for multiple comparisons were performed prior to post hoc testing with two-sided Wilcoxon signed-rank tests. Here, corrections were applied to control for family-wise type 1 error rate using Holm's sequential Bonferroni method.

Since the various conditions for practical reasons have not been tested in equal numbers, post-hoc analyses were carried out anyway where the within-subject mean effect sizes were calculated over a larger amount of stimulation patterns. This procedure was applied in order to use the data obtained as completely as possible. In addition we performed descriptive statistics including mean effect size and success rate.

\section{Results:}

\section{Standard CI Rehabilitation}

Because high consistency was found between the various scales the VAS scores of perceived tinnitus loudness, amount of discomfort, effect on life and extent of problems due to the tinnitus were averaged prior to the surgery, both before and after standard $\mathrm{Cl}$ rehabilitation and after 1 week of $\mathrm{Cl}$ deactivation and are shown in figure 2. No significant difference in the averaged VAS score was obtained between baseline and 1 week postoperative $(p=0.40)$. A gradual tinnitus reduction during $\mathrm{Cl}$ rehabilitation was obtained (data not shown) with a significant tinnitus reduction, compared to the baseline condition, after completing $\mathrm{Cl}$ rehabilitation $(p=0.0045)$. No significant tinnitus reduction, compared to baseline was obtained after 1 week of $\mathrm{Cl}$ deactivation following the $\mathrm{Cl}$ rehabilitation $(\mathrm{p}=0.24)$. 


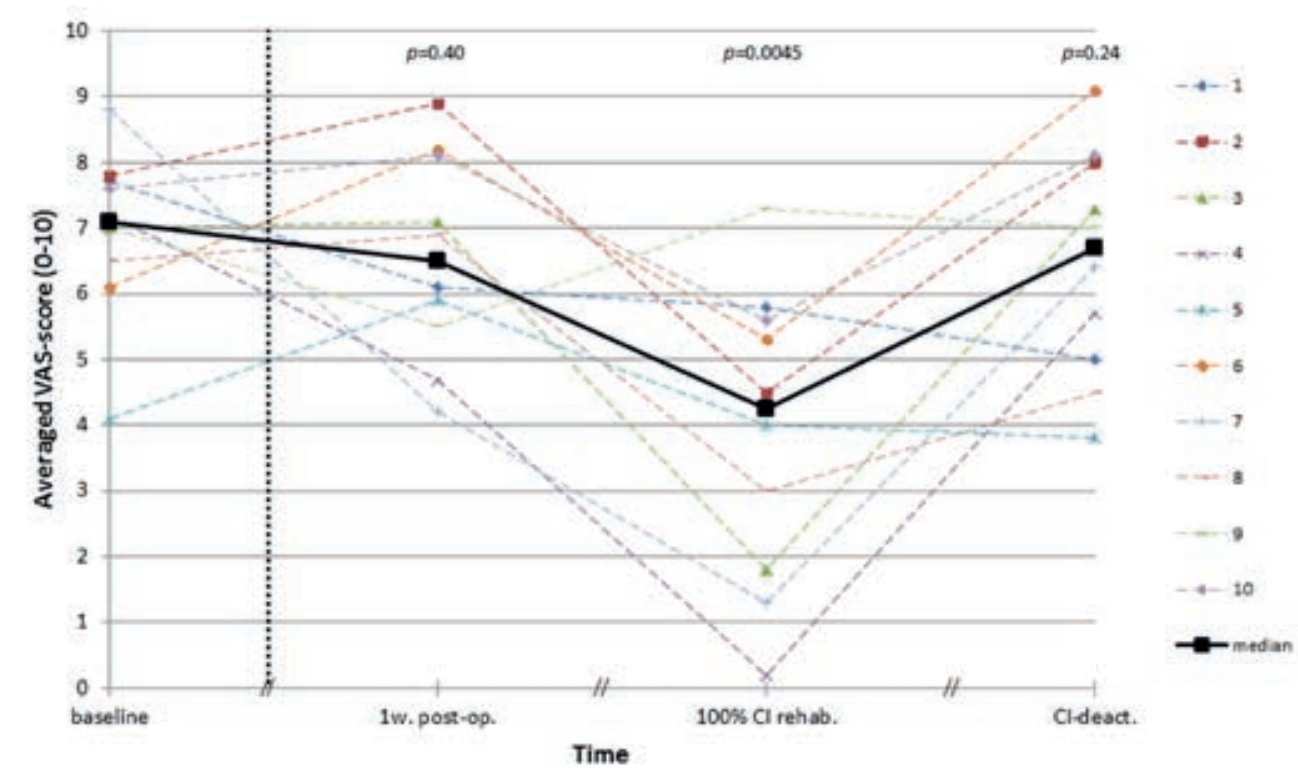

Fig.2: Effects of $\mathrm{Cl}$ surgery and standard $\mathrm{Cl}$ rehabilitation on perceived tinnitus loudness, amount of discomfort, effect on life and the extent of problems due to the tinnitus displayed as the averaged score on a VAS (0-10). The duration of the Cl rehabilitation was approximately 8 weeks. Individual results and medians are shown. Significant tinnitus reduction, compared to the baseline condition, was obtained after completing $\mathrm{Cl}$ rehabilitation.

Figure 3 shows the tinnitus loudness in $\mathrm{dB}$ SL matched via acoustic stimulation to the contralateral normal-hearing ear prior to the surgery, both before and after $\mathrm{Cl}$ rehabilitation and after 1 week of $\mathrm{Cl}$ deactivation. No significant difference in tinnitus loudness was obtained between baseline and 1 week postoperative $(p=0.45)$. Again, a gradual tinnitus reduction during $\mathrm{Cl}$ rehabilitation was obtained (data not shown) with a significant tinnitus reduction after completing $\mathrm{Cl}$ rehabilitation $(p=0.030)$, while no significant reduction was obtained between baseline and 1 week of $\mathrm{Cl}$ deactivation after the $\mathrm{Cl}$ rehabilitation $(p=0.14)$.

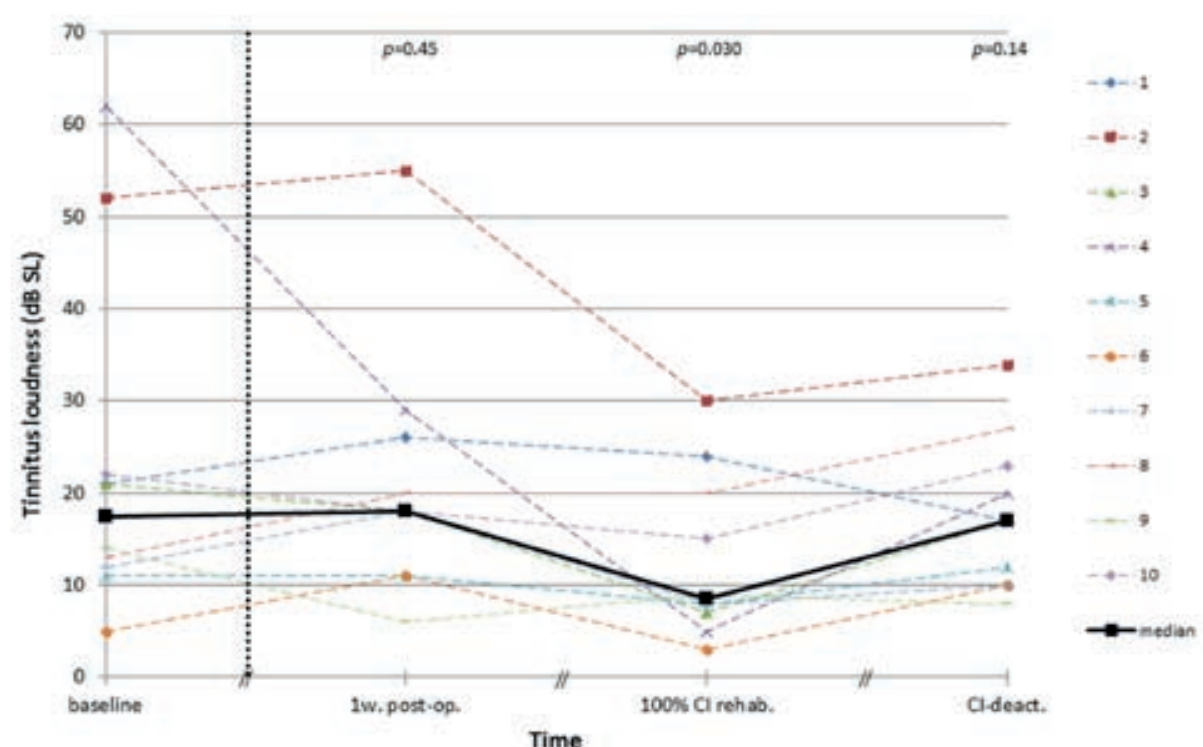

Fig.3: Effects of $\mathrm{Cl}$ surgery and standard $\mathrm{Cl}$ rehabilitation on tinnitus loudness matched via the contralateral normal-hearing ear. Individual results and medians are shown. Significant tinnitus reduction was obtained between baseline and after completing $\mathrm{Cl}$ rehabilitation.

The total THI and TQ scores prior to the surgery and both before and after standard $\mathrm{Cl}$ rehabilitation are shown in figure 4. No significant differences in tinnitus handicap (THI) and tinnitus distress (TQ) were obtained between baseline and 1 week postoperative $(p=0.38$ and $p=0.096$ respectively). Significant reductions were obtained between baseline and after completing $\mathrm{Cl}$ rehabilitation $(p=0.033$ and $p=$ 0.016 respectively).

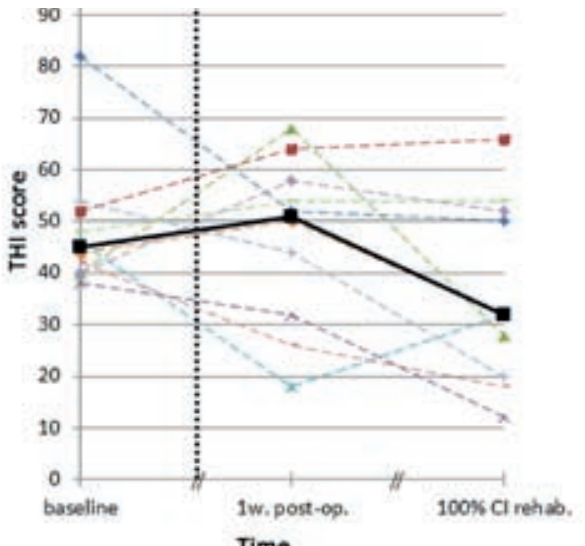

Time

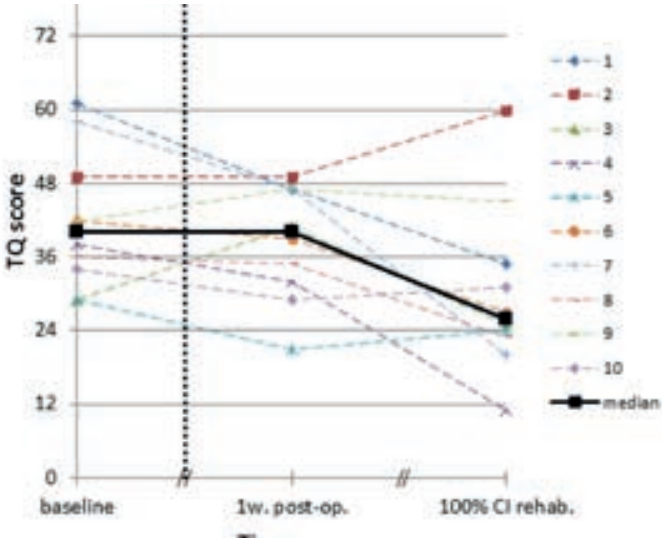

Iw. post
Fig.4: Effects of $\mathrm{Cl}$ surgery and standard CI rehabilitation on tinnitus handicap ( $\mathrm{THI}$; left graph) and tinnitus distress (TQ; right graph). Individual results and medians are shown. Significant reductions in tinnitus handicap and tinnitus distress were obtained between baseline and after completing $\mathrm{Cl}$ rehabilitation 
S1: pattern 23

\section{Fine-Tuning Procedure}

\section{General}

After clinical rehabilitation the $\mathrm{Cl}$ was deactivated for 1 week to minimize possible interfering effects of $\mathrm{C}$ rehabilitation. The fine-tuning procedure was thus started 1 week after Cl rehabilitation had ended. Table 3 shows the tinnitus pitch-matched electrode used for the different test days during the fine-tuning procedure. Tinnitus frequency seems quite stable across time for most patients but changes with time in some cases, resulting in a different tinnitus pitch-matched electrode.

Table 3: Pitch-matched electrode per test day (2, 3 or 4, based on patients' preference during the fine-tuning procedure.

\begin{tabular}{|c|c|c|c|c|c|c|c|c|}
\hline \multirow[t]{3}{*}{ Subject } & \multicolumn{4}{|c|}{ Tinnitus frequency during fine tuning procedure $(\mathrm{Hz})$} & \multicolumn{4}{|c|}{ Pitch Matched Electrode (PM elec.) } \\
\hline & \multicolumn{4}{|l|}{ Test day: } & \multicolumn{4}{|c|}{ Test day: } \\
\hline & 1 & 2 & 3 & 4 & 1 & 2 & 3 & 4 \\
\hline 1 & 2,820 (NB) & 3,550 (NB) & 1,190 (NB) & & 9 & 10 & 6 & \\
\hline 2 & 2,240 (NB) & 2,240 (NB) & 1,780 (NB) & & 8 & 8 & 7 & \\
\hline 3 & 4,470 (NB) & 4,000 (NB) & 4,000 (NB) & & 10 & 10 & 10 & \\
\hline 4 & 2,000 (NB) & 2,820 (NB) & & & 8 & 9 & & \\
\hline $5^{\mathrm{a}}$ & $7,100(\mathrm{~T})$ & $7,500(\mathrm{~T})$ & $8,000(\mathrm{~T})$ & $8,000(\mathrm{~T})$ & 10 & 10 & 10 & 10 \\
\hline 6 & 5,000 (NB) & 4,000 (NB) & 4,470 (NB) & 5,000 (NB) & 11 & 10 & 10 & 11 \\
\hline 7 & $1,250(\mathrm{~T})$ & $1,000(\mathrm{~T})$ & 800 (T) & $1,500(\mathrm{~T})$ & 6 & 6 & 5 & 7 \\
\hline 8 & 3,550 (NB) & 3,550 (NB) & 4,730 (NB) & 4,470 (NB) & 10 & 10 & 11 & 10 \\
\hline 9 & $2,820(\mathrm{~T})$ & $3,350(\mathrm{~T})$ & $1,780(\mathrm{~T})$ & & 9 & 9 & 7 & \\
\hline 10 & $3,000(\mathrm{~T})$ & $2,500(\mathrm{~T})$ & $3,000(\mathrm{~T})$ & $2,240(\mathrm{~T})$ & 9 & 8 & 9 & 8 \\
\hline
\end{tabular}

$N B=1 / 3$-octave narrowband, $T=$ pure tone, ${ }^{a}$ partial insertion with two extracochlear electrodes

Postoperative clinical examinations show that subject 5 had a partial insertion with two extracochlear electrodes, as confirmed by cone beam computed tomography. Therefore, the high pitch of the tinnitus was allocated to the most basal electrode which was still intracochlear (electrode 10).
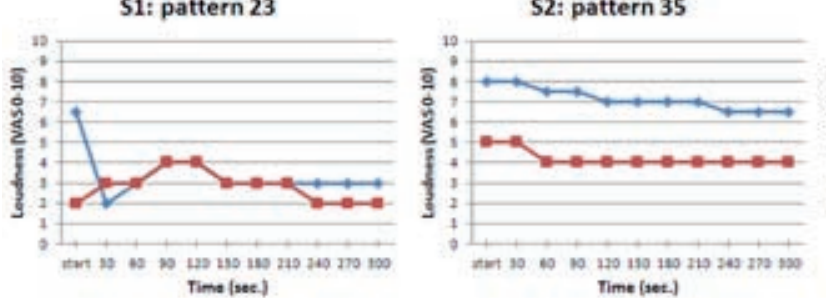

S4: pattern 3
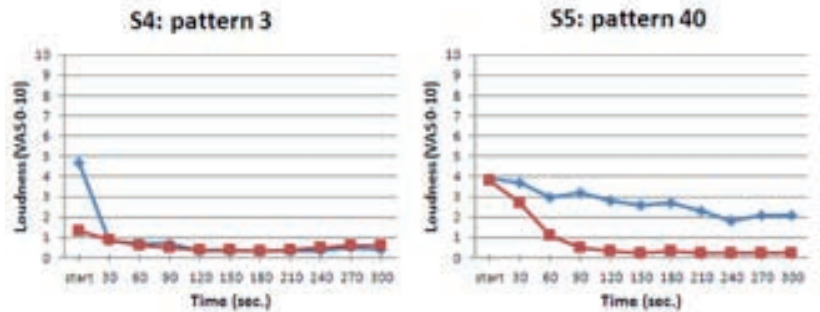

58: pattern 27
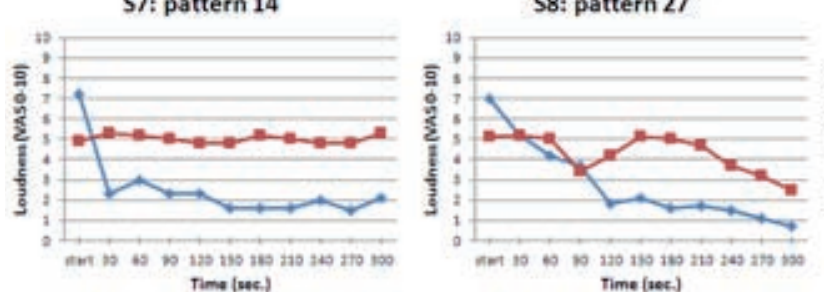

10: pattern 37

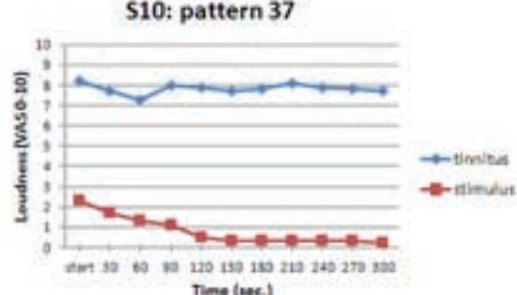

Fig.5: Representative examples of tested stimulation patterns and their effects on tinnitus and stimulus loudness perception. For each subject one stimulation pattern is shown. Tinnitus loudness on a VAS is indicated by diamonds, while the perceived stimulus loudness is indicated by squares. Patterns were tested for 5 min each.

Figure 5 shows representative examples of the effects on both tinnitus loudness and the perceived stimulus loudness of a tested stimulation pattern for each subject. Tinnitus reduction of at least $30 \%$ can be observed in 8 out of 10 subjects (fig. 5, subjects 1, 3-8 and 9). A significant loudness adaptation, that is a decrease in perceived stimulus loudness over time, is shown in 6 out of 10 subjects (fig. 5, subjects 4-6 and 8-10). In subjects 4 and 6 examples of 'the code of silence' are shown [Rubinstein et al., 2003; Arts et al., 2015]. Here, loudness adaptation occurred in combination with tinnitus suppression. For subjects 1 and 9 the same stimulation pattern is shown. Note that the effects on both tinnitus and stimulus loudness are different for both subjects. For subject 1, robust tinnitus suppression in the first seconds is shown after which the loudness stabilizes. No loudness adaptation occurs. On the other hand, in subject 9 , gradual tinnitus suppression together with complete loudness adaptation is observed. 
Individual results of the tested stimulation patterns are shown in table 4. Patterns 2, 10,18 and 26 were not tested in all subjects and patterns $8,16,24$ and 32 were not tested in 9 out of 10 subjects as these were technically not feasible due to the high tinnitus pitch and a limit to the overall (sum of each activated electrode) stimulation rate. Subject 2 did not complete the proposed patterns as she could tolerate only stimulation at apically located electrodes. As a compromise, some specifically designed patterns have been tested for this subject and several others who could manage the necessary measurement time (Appendix 2). Results from subject 2 were excluded from analysis of the data obtained from the proposed patterns.

General analysis of the 48 proposed patterns (Appendix 1) shows that in 153 out of the 365 patterns tested in total (41.9\%) the tinnitus loudness was reduced by at least $30 \%$. For 1 out of 365 tested patterns the tinnitus loudness increased at least by $30 \%$. Luckily, the negative effect on tinnitus loudness disappeared within seconds after deactivation. Figure 6 gives an overview of the observed effects for all subjects, showing that 8 out of 10 subjects $(80 \%)$ were responders, experiencing a tinnitus reduction in at least one condition by $30 \%$ or more.

\section{Effect of Loudness}

No significant difference in tinnitus reduction was found between soft stimulation (conditions with a VAS loudness 2), medium stimulation (conditions with a VAS loudness 5 ) and loud stimulation (conditions with a VAS loudness 7, Friedman test: $p=0.097$ ). Since loud stimulation was tested only twice and soft stimulation was observed to have a considerably lower mean effect size compared to both medium and loud stimulation, soft stimulation was compared to medium to loud stimulation (conditions with a VAS loudness 5 or 7). Here, medium to loud stimulation reduced tinnitus significantly better than soft stimulation $(p=0.038)$. Furthermore, with loud stimulation a success rate was obtained of 9 out of 18 patterns tested (50.0\%). However, tinnitus suppression could also be achieved when stimulus loudness adaptation occurred.

\section{Effect of Stimulation Site}

The effect of looped electrical stimulation on tinnitus did not differ significantly between stimulation on the most basal located electrode, the most apical located electrode, the tinnitus pitch-matched electrode together with the two adjacent electrodes and stimulation at these two adjacent electrodes without the tinnitus pitch-matched electrode (Friedman test, $p=0.10$ ). In this analysis, each condition was tested for only two stimulation patterns. Electrical stimulation at the two adjacent electrodes of the tinnitus pitchmatched electrode shows the highest mean effect size with a success rate of 9 out of 18 patterns tested $(50 \%)$, while electrical stimulation at the most apical located electrode shows the lowest mean effect size. The effect of stimulation at the tinnitus pitch-matched electrode on tinnitus did not differ significantly from electrical stimulation at all intracochlear electrodes $(p=0.59)$. Finally, the effect of narrowband electrical stimulation (i.e., one single electrode) on tinnitus did not differ significantly from broadband electrical stimulation (i.e., more than one single electrode, $p=0.31$ ).

\section{Effect of Modulation}

Again, no significant difference in tinnitus reduction was found between fixed amplitude (no modulation) random, burst and sine wave amplitude modulation (Friedman test, $p=0.48$ ). In this analysis, each condition was tested only twice. Electrical stimulation with burst amplitude modulation shows a considerable higher mean effect size with a success rate of 10 out of 18 patterns tested (55.6\%), while unmodulated electricalstimulation shows the lowestmean effect size. Withtheaddition of supplementary data, random amplitude modulation showed again no significant difference in tinnitus reduction 


\section{Discussion:}

\section{General}

Our study shows a significant tinnitus reduction after completing standard $\mathrm{Cl}$ rehabilitation. This effect disappeared after 1 week of $\mathrm{Cl}$ deactivation. No effect of the $\mathrm{Cl}$ surgery itself on tinnitus was observed. In $80 \%$ of our subjects at least one successful tinnitus-suppressing pattern could be found in the fine-tuning procedure. Results show that medium to loud stimulation patterns reduce tinnitus significantly better than soft stimulation patterns. No significant effects of stimulation site, amplitude modulation, polarity or pulse rate were observed.

\section{Standard Cl Rehabilitation}

The observed effects on tinnitus after completing the standard $\mathrm{Cl}$ rehabilitation phase are in line with previous studies [Van de Heyning et al., 2008; Kleinjung et al., 2009; Arndt et al., 2010; Buechner et al., 2010; Palau et al., 2010; Jacob et al., 2011; Kleine Punte et al., 2011; Firszt et al., 2012b; Ramos et al., 2012; Hansen et al., 2013; Távora-Vieira et al., 2013]. Our results show a decrease in tinnitus perception over the approximately 8 weeks of $\mathrm{Cl}$ rehabilitation that is not caused by the surgery itself, which is consistent with an earlier case report [Gartrell et al., 2014]. Based on the findings of previous studies, tinnitus reduction may be further optimized by prolonged $\mathrm{Cl}$ use. Suppressive effects on tinnitus were partially undone by $\mathrm{Cl}$ deactivation which is also consistent with previous studies [Van de Heyning et al., 2008; Arndt et al., 2010].

\section{Fine-Tuning Procedure}

During the fine-tuning procedure, $80 \%$ of the subjects were shown to be responders to the $\mathrm{Tl}$, experiencing a tinnitus reduction of at least $30 \%$ with at least one stimulation pattern that does not encode acoustic sounds. Therefore, our results show that it is possible to reduce tinnitus with looped intracochlear electrical stimulation, at least for minutes. This finding is consistent with previous studies [Rubinstein et al., 2003; Zeng et al., 2011; Chang and Zeng, 2012; Arts et al., 2015] although we found a relatively high percentage of responders. Because the optimal stimulation pattern is highly subject-specific, the fairly large success rate may be explained by the large number of conditions tested per subject. Our overall response rate was $42 \%$ (153/365 conditions tested) while the overall response rate found in Chang and Zeng [2012] was 37\% (83/227 conditions tested) and 35\% (37/107 conditions tested) by Arts et al. [2015].

As discussed before, Subjects 4 and 6 show tinnitus suppression in combination with stimulus loudness adaptation (fig. 5). In both subjects, complete silence was achieved within seconds of stimulation. The silence during stimulation indicates that inaudible electrical stimulation with looped patterns is able to suppress tinnitus successfully, at least in some cases. Furthermore, it refutes the idea that a shift in attention from the tinnitus to another sound (whether environmental sounds in standard $\mathrm{Cl}$ or stimulus perceptions in $\mathrm{Tl}$ ) is the responsible mechanism for tinnitus suppression via intracochlear stimulation.

\section{Effect of Loudness}

Results show that medium to loud stimulation reduces tinnitus significantly better than soft stimulation. Moreover, the mean percentage tinnitus reduction was considerably lower for soft stimulation compared to both medium and loud stimulation. In our earlier study [Arts et al., 2015] we found no significant effect of stimulation level on tinnitus reduction, although a trend was found. Chang and Zeng [2012] concluded that loud stimulation leads to more successful cases of tinnitus reduction than soft sound, although no significant effect of stimulation level was found on the overall degree of tinnitus reduction. Masking and 
an attention shift from the tinnitus to the stimulus could both be possible explanations for better tinnitus reduction in medium to loud stimulation compared to soft stimulation.

\section{Effect of Stimulation Site}

Electrical stimulation via the tinnitus pitch-matched electrode did not have a significantly different effect on tinnitus than stimulation at all available electrodes. This is consistent with a previous study in bilaterally hearing-impaired $\mathrm{Cl}$ users [Arts et al. 2015]. However, caution is required in interpreting these results, as there is some uncertainty in the determination of the tinnitus pitch matched electrode via the used method. For example, no postoperative $X$-rays were available from each subject to determine the tinnitus pitch-matched electrode on the basis of the tinnitus pitch and electrode location [Boëx et al., 2006; Vermeire et al., 2008]. Furthermore, it is not exactly known whether stimulation targets the nerve fibers at the level of the organ of Corti or the spiral ganglion cells within Rosenthal's canal. As Rosenthal's canal is not linearly related to the organ of Corti [Kawano et al., 1996] it is unclear which frequency-position function is appropriate for intracochlear electrical stimulation [Greenwood, 1990; Sridhar et al., 2006; Stakhovskaya et al., 2007; Schatzer et al., 2014]. Finally, mechanisms like spread of excitation (Hughes et al. 2013) including cross-turn stimulation [Frijns et al., 2001; Boëx et al., 2006] and channel interaction [Abbas et al., 2004, Undurraga et al., 2012] and frequency-place map reorganization [Carlyon et al., 2010; Reiss et al., 2014] may influence the frequency-position prediction, and may thus affect which electrode is the tinnitus pitch-matched electrode.

\section{Effect of Modulation}

No significant differences in tinnitus suppression were observed between the applied amplitude modulations. However, the mean percentage tinnitus reduction was highest for burst amplitude modulation compared to fixed, random and sine wave amplitude modulation. As far as the authors know, burst stimulation has never been applied for tinnitus reduction via intracochlear electrical stimulation. However, stimulation with pulse trains has been applied by vagus nerve stimulation [Engineer et al., 2011], external ear canal stimulation [Mielczarek et al., 2013] and transcranial magnetic stimulation [Kim et al., 2014] as an experiment for tinnitus suppression with varying results. Therefore, burst stimulation seems a promising option for tinnitus suppression, but future experiments will have to show whether current results can be replicated or even optimized.

\section{Effect of Polarity}

Charge-balanced biphasic pulses were applied in the current study as direct current stimulation could damage tissue [Aran, 1977; Hazell et al., 1993; Shepherd et al., 1999; Bahmer and Baumann, 2013]. Our results show no significant difference between cathodic and anodic first biphasic charge-balanced electrical stimulation. However, caution is required as anodic first stimulation was applied only twice per subject. A difference in tinnitus suppression between cathodic and anodic first charge-balanced electrical stimulation was initially expected as prior studies showed that anodic stimulation could be more sensitive than cathodic stimulation [Undurraga et al., 2010, 2013; Carlyon et al., 2013]. The cathodic phase is thought to stimulate the peripheral process while the anodic phase probably excites the nerve fibers more centrally [Rattay et al., 2001]. Cazals et al. [1978] found tinnitus suppression with anodic extracochlear stimulation while cathodic stimulation evoked auditory sensation.

\section{Effect of Rate}

The current study found no significant difference in tinnitus reduction between the stimulation rates applied. There was also no difference found between the effect of low ( $<2,000 \mathrm{pps})$ and high ( $\geq 2,000 \mathrm{pps})$ stimulation rates on tinnitus suppression, which is consistent with a previous study [Chang and Zeng,
2012]. Rubinstein et al. [2003] used a high stimulation rate (4,800 pps) at the tinnitus pitch-matched electrode to mimic spontaneous neural activity for tinnitus suppression. In 2 out of 3 subjects the tinnitus was suppressed, 1 of these subjects perceived tinnitus suppression in combination with complete adaptation to the stimulus percept (the code of silence). However, in contrast, Zeng et al. [2011] found tinnitus suppression in combination with partial adaptation of the stimulus using low-rate (100 pps) stimulation at the most apical located electrode in an SSD subject. Thus, the optimal stimulation rate seems to be subject-specific.

\section{Limitations}

In the current prospective clinical trial caution is required in interpreting the results. A (double-blind) randomized controlled trial would for example be desirable to control for possible placebo effects. However, a 'placebo Cl' treatment raises serious ethical concern. As a compromise, the current study was designed as a crossover trial to subsequently examine the long-term effects of both the standard clinical $\mathrm{Cl}$ and the experimental TI. Long-term results will be reported in a later stage (part II). Furthermore, the limited sample size does not allow making robust statements about the effect of surgery on tinnitus or about the effectiveness of intracochlear electrical stimulation to suppress tinnitus. Finally, the exploratory analyses on the short-term effects on tinnitus suppression during the fine-tuning procedure must be interpreted with caution. Further research is therefore recommended.

Studying the SSD population may possibly limit the applicability of the findings to the wider $\mathrm{Cl}$ population. However, the current study investigated the possibility of suppressing tinnitus with looped electrical stimulation in subjects with clearly lateralized tinnitus to the deaf ear, and the periphery of the deaf ear in the studied population seems to be the same as for the 'conventional' bilaterally deaf population. The similarity between the deaf ear in the SSD population and in the bilaterally deaf population is supported by comparable outcomes of the standard clinical $\mathrm{Cl}$ between these populations on for example electric dynamic ranges, pitch saturation limit, speech perception and tinnitus [Firszt et al., 2012a; Olze et al., 2012; Vermeire and Lawson, 2012; Schatzer et al., 2014].

In conclusion, current results showed a tinnitus reduction during standard clinical $\mathrm{Cl}$ rehabilitation while the $\mathrm{Cl}$ surgery itself had no positive or negative effect on tinnitus. The baseline (original) tinnitus loudness was restored after 1 week of $\mathrm{Cl}$ deactivation. These findings corroborate the hypothesis that tinnitus is related to unwanted neuroplasticity related to hearing loss and indicate that tinnitus can be suppressed at least for minutes by looped intracochlear electrical stimulation. Optimal stimuli for tinnitus suppression were observed to be subject-specific, which means that the fine-tuning procedure needs to be customized for each individual user. However, medium to loud electrical stimuli were shown to suppress tinnitus significantly better than soft stimuli, which partly can be explained by the masking effect. A future prospective longitudinal crossover trial with the current subjects will investigate the possible benefits of electrical stimulation on tinnitus suppression in the long run.

\section{Appendices:}

The current study tested a wide variability in stimulus characteristics, with a total of 48 different stimulation patterns.

The amplitude, expressed as the perceived stimulus loudness on a VAS, was initially set to 2 (soft), 5 (medium) or 7 (loud). Loud stimulation would be the most likely to mask tinnitus whereas soft stimulation would have the highest stimulus comfort. 
To vary the anatomical location inside the cochlea, different electrodes were activated. Tested stimulation sites were: PM elec. (the tinnitus pitch-matched electrode), all (all available electrodes), basal elec. (the most basal located electrode), apical elec. (the most apical located electrode), PM elec. + two adjacent elec. (the tinnitus pitch-matched electrode together with the two adjacent electrodes) and two adjacent elec. of PM elec. [the two electrodes adjacent to (but without) the tinnitus pitch-matched electrode]. Although Zeng et al. [2011] found in an SSD subject that stimulation in the apical region of the cochlea suppresses tinnitus better than stimulation in the basal region, Rubinstein et al. [2003] reported on tinnitus suppression stimulating the electrode that was pitch matched to the tinnitus. Chang and Zeng [2012] and Arts et al. [in press] found no significant effect of stimulation place on tinnitus suppression.

Furthermore, four amplitude modulations were tested: fixed (no modulation), sine wave [20 sine waves per loop, with a modulation depth of $\left.(30 / 128)^{*} 100 \%\right]$, burst (per loop, trains of five consecutive pulses followed by a gap with a duration depending on the used pulse rate) and random (random amplitudes with a modulation depth of $100 \%$, resulting in a minimum modulation frequency of $34 \mathrm{~Hz}$ at a stimulation rate of 200 pps, which resembles speech envelope information [Rosen, 1992]; fig. 1). Each loop consisted of 128 pulses, with a duration that is depending on the used stimulation rate.

Cathodic as well as anodic first biphasic stimulation were tested. Although cathodic first biphasic stimulation is used for speech processing in Cls and the suppressive effect of speech processing on tinnitus is known, reports on tinnitus suppression with intracochlear anodic first biphasic stimulation are lacking.

Furthermore, stimulation patterns included pulse rates of 200,750 and 5,000 pps/channel and a stimulation rate equal to the frequency of the perceived tinnitus in Hertz. These stimulation rates were tested as Zeng et al. [2011] reported on complete tinnitus suppression with low-rate (100 pps) stimulation on the most apically located electrode while Rubinstein et al. [2003] reported on complete tinnitus suppression with high-rate (4,800 pps) stimulation at the tinnitus pitch-matched electrode. The high stimulation rate causes pseudostochastic responses since the pulse interval duration is less than the absolute refractory period of the neurons. These pseudostochastic responses could in theory mimic (not synchronous) spontaneous activity [Rubinstein et al., 1999]. A stimulation rate equal to the frequency of the perceived tinnitus in Hertz was tested in an effort to mimic acoustic stimulation where the frequency of the traveling wave on the basilar membrane is analogous to the frequency of the opening of the $\mathrm{K}^{+}$ channels in the stereocilia.

The used phase duration was fixed between the active electrodes within the patterns tested, but not within subjects (because of the limited current level and loudness balancing at the maximum comfortable level) or between subjects (technically not feasible).
Appendix 1: Tested looped Stimulation Patterns.

\begin{tabular}{|c|c|c|c|c|c|}
\hline Pattern & $\begin{array}{l}\text { Amplitude } \\
\text { (VAS-loudness } \\
0-10 \text { ) }\end{array}$ & Electrode(s) & $\begin{array}{l}\text { Amplitude } \\
\text { Modulation }\end{array}$ & $\begin{array}{l}\text { Polarity } \\
\text { (cathodic/anodic } \\
\text { first) }\end{array}$ & $\begin{array}{l}\text { Stimulation } \\
\text { Rate (pps/ } \\
\text { channel) }\end{array}$ \\
\hline 1 & 2 & PM elec. & Fixed & Cathodic & 5,000 \\
\hline 2 & 2 & All & Fixed & Cathodic & 5,000 \\
\hline 3 & 2 & PM elec. & Fixed & Cathodic & 750 \\
\hline 4 & 2 & All & Fixed & Cathodic & 750 \\
\hline 5 & 2 & PM elec. & Fixed & Cathodic & 200 \\
\hline 6 & 2 & All & Fixed & Cathodic & 200 \\
\hline 7 & 2 & PM elec. & Fixed & Cathodic & PM \\
\hline 8 & 2 & All & Fixed & Cathodic & PM \\
\hline 9 & 5 & PM elec. & Fixed & Cathodic & 5,000 \\
\hline 10 & 5 & All & Fixed & Cathodic & 5,000 \\
\hline 11 & 5 & PM elec. & Fixed & Cathodic & 750 \\
\hline 12 & 5 & All & Fixed & Cathodic & 750 \\
\hline 13 & 5 & PM elec. & Fixed & Cathodic & 200 \\
\hline 14 & 5 & All & Fixed & Cathodic & 200 \\
\hline 15 & 5 & PM elec. & Fixed & Cathodic & PM \\
\hline 16 & 5 & All & Fixed & Cathodic & PM \\
\hline 17 & 2 & PM elec. & Random & Cathodic & 5,000 \\
\hline 18 & 2 & All & Random & Cathodic & 5,000 \\
\hline 19 & 2 & PM elec. & Random & Cathodic & 750 \\
\hline 20 & 2 & All & Random & Cathodic & 750 \\
\hline 21 & 2 & PM elec. & Random & Cathodic & 200 \\
\hline 22 & 2 & All & Random & Cathodic & 200 \\
\hline 23 & 2 & PM elec. & Random & Cathodic & PM \\
\hline 24 & 2 & All & Random & Cathodic & PM \\
\hline 25 & 5 & PM elec. & Random & Cathodic & 5,000 \\
\hline 26 & 5 & All & Random & Cathodic & 5,000 \\
\hline 27 & 5 & PM elec. & Random & Cathodic & 750 \\
\hline 28 & 5 & All & Random & Cathodic & 750 \\
\hline 29 & 5 & PM elec. & Random & Cathodic & 200 \\
\hline 30 & 5 & All & Random & Cathodic & 200 \\
\hline 31 & 5 & PM elec. & Random & Cathodic & PM \\
\hline 32 & 5 & All & Random & Cathodic & PM \\
\hline 33 & 5 & Basal elec. & Random & Cathodic & 5,000 \\
\hline 34 & 5 & Basal elec. & Random & Cathodic & PM \\
\hline 35 & 5 & Apical elec. & Random & Cathodic & 5,000 \\
\hline 36 & 5 & Apical elec. & Random & Cathodic & PM \\
\hline 37 & 5 & PM elec. + two adjacent elec. & Random & Cathodic & 5,000 \\
\hline 38 & 5 & PM elec. + two adjacent elec. & Random & Cathodic & PM \\
\hline 39 & 5 & Two adjacent elec. of PM elec. & Random & Cathodic & 5,000 \\
\hline 40 & 5 & Two adjacent elec. of PM elec. & Random & Cathodic & PM \\
\hline 41 & 7 & PM elec. & Random & Cathodic & 5,000 \\
\hline 42 & 7 & PM elec. & Random & Cathodic & PM \\
\hline 43 & 5 & PM elec. & Random & Anodic & 5,000 \\
\hline 44 & 5 & PM elec. & Random & Anodic & PM \\
\hline 45 & 5 & PM elec. & Burst & Cathodic & 5,000 \\
\hline 46 & 5 & PM elec. & Burst & Cathodic & PM \\
\hline 47 & 5 & PM elec. & Sine wave & Cathodic & 5,000 \\
\hline 48 & 5 & PM elec. & Sine wave & Cathodic & PM \\
\hline
\end{tabular}


Appendix 2: Additional Patterns Tested. Marked patterns were selected as potential tinnitus suppressors which will be utilized in the longitudinal study following the present acute study.

\begin{tabular}{|c|c|c|c|c|c|c|}
\hline Subject & $\begin{array}{l}\text { Amplitude } \\
\text { (VAS-loudness } \\
0-10 \text { ) }\end{array}$ & Electrode(s) & $\begin{array}{l}\text { Amplitude } \\
\text { Modulation }\end{array}$ & $\begin{array}{l}\text { Polarity } \\
\text { (first cathodic/ } \\
\text { anodic) }\end{array}$ & $\begin{array}{l}\text { Stimulation } \\
\text { Rate (pps/ } \\
\text { channel) }\end{array}$ & Effectiveness \\
\hline 2 & 5 & Elec. $1 \& 2$ & Fixed & Cathodic & 200 & $=$ \\
\hline 2 & 5 & Elec. 1-3 & Fixed & Cathodic & 750 & $=$ \\
\hline 2 & 5 & Elec. 2 \& 4 & Fixed & Cathodic & 200 & $=$ \\
\hline 2 & 5 & Elec. 3 & Fixed & Anodic & 5,000 & $=$ \\
\hline 2 & 5 & Apical & Sine wave & Anodic & 200 & $=$ \\
\hline 2 & 5 & Elec. 1 \& 2 & Fixed & Cathodic & 5,000 & $=$ \\
\hline 2 & 5 & Apical & Fixed & Cathodic & 200 & $=$ \\
\hline 2 & 5 & Apical & Burst & Anodic & 5,000 & $=$ \\
\hline 2 & 5 & Elec. 1-4 & Fixed & Cathodic & 750 & $=$ \\
\hline 2 & 6 & Elec. 1-8 & Random & Cathodic & 750 & $=$ \\
\hline 2 & 5 & Elec. 1-8 & Fixed & Cathodic & 750 & $=$ \\
\hline 3 & 2 & Two adjacent elec. of PM & Random & Cathodic & PM & + \\
\hline 3 & 2 & PM + two adjacent & Random & Cathodic & PM & + \\
\hline 5 & 5 & Two adjacent elec. of PM & Random & Cathodic & 750 & $=$ \\
\hline 5 & 5 & Basal & Random & Cathodic & 13,000 & $=$ \\
\hline 5 & 5 & Elec. 9 & Random & Cathodic & 13,000 & $=$ \\
\hline 5 & 5 & Elec. 8 & Random & Cathodic & 18,000 & $=$ \\
\hline 5 & 5 & PM elec. & Fixed & Cathodic & PM & $=$ \\
\hline 5 & 5 & Elec. 9-11 & Random & Cathodic & 5,000 & $=$ \\
\hline 5 & 5 & Elec. 10 \& 11 & Fixed & Cathodic & 750 & + \\
\hline 5 & 6 & Elec. 9 \& 11 & Sine wave & Cathodic & 200 & $=$ \\
\hline 5 & 3 & Basal & Sine wave & Anodic & PM & $=$ \\
\hline 5 & 3 & Elec. $1,9,10$ & Fixed & Cathodic & 5,000 & $=$ \\
\hline 5 & 5 & Elec. 7-11 & Sine wave & Cathodic & 200 & $=$ \\
\hline 5 & 5 & Basal & Sine wave & Cathodic & 10,000 & + \\
\hline 5 & 5 & Basal & Random & Cathodic & 10,000 & $=$ \\
\hline 5 & 5 & Basal & Fixed & Anodic & 10,000 & $=$ \\
\hline 5 & 4 & Basal & Fixed & Cathodic & 16,000 & $=$ \\
\hline 5 & 4 & Basal & Random & Anodic & 16,000 & $=$ \\
\hline 5 & 5 & Elec. $9 \& 11$ & Random & Anodic & PM & $=$ \\
\hline 5 & 5 & Elec. $9 \& 11$ & Sine wave & Cathodic & PM & $=$ \\
\hline 5 & 4 & Elec. $10 \& 11$ & Random & Cathodic & 5,000 & $=$ \\
\hline 5 & 2 & Basal & Fixed & Cathodic & 100 & $=$ \\
\hline 5 & 5 & Elec. 9-11 & Burst & Cathodic & 750 & $=$ \\
\hline 9 & 5 & Elec. 8 & Burst & Cathodic & PM & $=$ \\
\hline 9 & 5 & Elec. 8 & Sine wave & Cathodic & PM & $=$ \\
\hline 9 & 5 & Elec. 7-9 & Fixed & Cathodic & PM & + \\
\hline 9 & 5 & Elec. 7-9 & Random & Anodic & PM & + \\
\hline 10 & 7 & All & Fixed & Anodic & 750 & $=$ \\
\hline 10 & 4 & All & Fixed & Anodic & 750 & $=$ \\
\hline 10 & 4 & PM elec. & Fixed & Cathodic & PM & $=$ \\
\hline 10 & 2 & PM elec. & Random & Cathodic & 750 & $=$ \\
\hline
\end{tabular}

$=$ aggravation of at least $30 \%$, $+=$ reduction of at least $30 \%,++=$ reduction of at least $70 \%,{ }^{\prime}=\prime=$ less than $30 \%$

aggravation/reduction

\section{Acknowledgements:}

The authors would like to thank Katrien Vermeire, MSc, PhD, for her contribution during the development of the test protocol, M. Janssen, MSc, for the statistical support, the independent Data Safety Monitoring Board (Jan Wouter Brunings, MD, Fons Kessels, MD, MSc, and Dyon Scheijen, MSc) for overseeing the safety of the included subjects and two anonymous reviewers for their suggestions and comments. Moreover, the authors are very grateful to the participants for their effort during data collection. This study was financially supported by MED-EL (MED-EL Corp., Innsbruck, Austria) and the Dutch Heinsius Houbolt Foundation.

\section{Disclosure Statement:}

This work was supported by a research grant from MED-EL Corp., Innsbruck, Austria. The funding source had no involvement in the study design; in the collection, analysis and interpretation of data; in the writing of the report; or in the decision to submit the article for publication. The authors declare that they have no conflict of interest. 


\section{References:}

Abbas PJ, Hughes ML, Brown CJ, Miller CA, South H: Channel interaction in cochlear implant users evaluated using the electrically evoked compound action potential. Audiol Neurotol 2004;9:203-213.

Aran JM: Neural correlates of electrically-induced cochlear dysfunction. Clinical Otolaryngology 1977;2:305-310.

Arndt S, Aschendorff A, Laszig R, Beck R, Schild C, Kroeger S, Ihorst G, Wesarg T: Comparison of pseudobinaural hearing to real binaural hearing rehabilitation after cochlear implantation in patients with unilateral deafness and tinnitus. Otol Neurotol 2010;32:39-47.

Arts RA, George EL, Chenault MN, Stokroos RJ: Optimizing intracochlear electrical stimulation to suppress tinnitus. Ear Hear 2015;36:125-135.

Arts RAGJ, George ELJ, Griessner A, Zierhofer C, Stokroos RJ: Long term effects of intracochlear electrical stimulation with looped patterns on tinnitus: a case report. Ear Noise Throat J, in press.

Arts RA, George EL, Stokroos RJ, Vermeire K: Review: Cochlear implants as a treatment of tinnitus in single-sided deafness. Curr Opin Otolaryngol Head Neck Surg 2012;20:398-403.

Aschendorff A, Pabst G, Klenzner T, Laszig R: Tinnitus in cochlear implant users: The Freiburg Experience. Int Tinnitus J 1998;2:162-164.

Baguley D, McFerran D, Hall D: Tinnitus. Lancet 2013;382:1600-1607.

Bahmer A, Baumann U: Effects of electrical pulse polarity shape on intra cochlear neural responses in humans: Triphasic pulses with cathodic second phase. Hearing Res 2013;306:123-130.

Beck AT, Beamesderfer A: Assessment of depression: The Depression Inventory. Psychological Measurements in Psychopharmacology. Mod Probl Pharmacopsychiat 1974;7:151-169.

Beck AT, Ward CH, Mendelson M, Mock J, Erbaugh J: An inventory for measuring depression. Archives of General Psychiatry 1961;4:561-571

Berliner KI, Shelton C, Hitselberger WE, Luxford WM: Acoustic tumors: effect of surgical removal on tinnitus. Am J Otol 1992;13:13-7.

Blasco MA, Redleaf MI: Cochlear implantation in unilateral sudden deafness improves tinnitus and speech comprehension: meta-analysis and systematic review. Otol Neurotol 2014 [Epub ahead of print]

Boëx C, Baud L, Cosendai G, Sigrist A, Kós M-I, Pelizzone M: Acoustic to electric pitch comparisons in cochlear implant subjects with residual hearing. J Assoc Res Otolaryngol 2006;7:110-124.

Bouman TK, Luteijn F, Albersnagel FA, van der Ploeg FAE: Enige ervaringen met de Beck Depression Inventory. Gedrag - Tijdschrift voor psychologie 1985;13:13-24.
Buechner A, Brendel M, Lesinski-Schiedat A, Wenzel G, Frohne-Buechner C, Jaeger B, Lenarz T: Cochlear implantation in unilateral deaf subjects associated with ipsilateral tinnitus. Otol Neurotol 2010;31:1381 1385.

Carlyon RP, Deeks JM, Macherey O: Polarity effects on place pitch and loudness for three cochlearimplant designs and at different cochlear sites. J Acoust Soc Am 2013;134(1):503-509.

Carlyon RP, Macherey O, Frijns JHM, Axon PR, Kalkman RK, Boyle P, Baguley DM, Briggs J, Deeks JM, Briaire J, Barreau X, Dauman R: Pitch comparison between electrical stimulation of a cochlear implan and acoustic stimuli presented to a normal-hearing contralateral ear. J Assoc Res Otolaryngol 2010;11:625-640

Cazals Y, Negrevergine M, Aran J-M: Electrical stimulation of the cochlea in man: hearing induction and tinnitus suppression. J Am Aud Soc 1978;3(5):209-13.

Chang JE, Zeng F-G: Tinnitus suppression by electric stimulation of the auditory nerve. Front Syst Neurosci DOI: 10.3389/2012/00019.

Cima RF, Maes IH, Joore MA, Scheyen DJ, El Refaie A, Baguley DM, Anteunis LJ, van Breukelen GJ, Vlaeyen JW: Specialised treatment based on cognitive behaviour therapy versus usual care for tinnitus: randomized controlled trial. Lancet 2012;379(9830):1951-9,

Davis PB, Paki B, Hanley PJ: Neuromonics tinnitus treatment: third clinical trial. Ear Hear 2007;28:242 259

de Carvalho GM, Guimaraes AC, Duarte ASM, Muranaka EB, Soki MN, Martins RSZ, Bianchini WA, Paschoal JR, Castilho AM: Hearing preservation after cochlear implantation: UNICAMP outcomes. Int J Otolaryngol DOI: 10.1155/2013/107186

Di Nardo W, Cantore I, Cianfrone F, Melillo P, Scorpecci A, Paludetti G: Tinnitus modifications after cochlear implantation. Eur Arch Otorhinolaryngol 2007:264:1145-1149.

Eggermont JJ, Roberts LE: The neuroscience of tinnitus: understanding abnormal and normal perception. Front Syst Neurosci DOI: 10.3389/2012/00053.

Engineer ND, Riley JR, Seale JD, Vrana WA, Shetake JA, Sudanagunta SP, Borland MS, Kilgard MP: Reversing pathological neural activity using targeted plasticity. Nature 2011;470(7332):101-104

Erlandsson S, Ringdahl A, Hutchins T, Carlsson SG: Treatment of tinnitus: a controlled comparison of masking and placebo. Br J Audiol 1987:21(2):37-44.

Fioretti A, Peri G, Eibenstein A: Suppression of tinnitus in a patient with unilateral sudden hearing loss: a case report. Case Reports in Otolaryngology DOI: 10.1155/2012/210707.

Firszt JB, Holden LK, Reeder RM, Cowdrey L, King S: Cochlear implantation in adults with asymmetric hearing loss. Ear Hear 2012a;33(4):521-33. 
Jacob R, Stelzig Y, Nopp P, Schleich P: Audiologische Ergebnisse mit Cochleaimplantat bei einseitiger Taubheit. HNO 2011;59:453-460

adults with unilateral deafness: a pilot study. Otol Neurotol 2012b;33:1339-1346.

Frijns JHM, Briaire JJ, Grote JJ: The importance of human cochlear anatomy for the results of the modiolus-hugging multichannel cochlear implants. Otol Neurotol 2001;22:340-349.

Gartrell BC, Jones HG, Kan A, Buhr-Lawler M, Gubbels SP, Litovsky RY: Investigating long-term effects of cochlear implantation in single-sided deafness: a best practice model for longitudinal assessment of spatial hearing abilities and tinnitus handicap. Otol Neurotol 2014;35:1525-1532.

Gilles A, Van Hal G, De Ridder D, Wouters K, Van de Heyning P: Epidemiology of noise-induced tinnitu and the attitudes and beliefs towards noise and hearing protection in adolescence. PLOS ONE DOI:

10.1371/journal.pone.0070297.

Greenwood DD: A cochlear frequency-position function for several species - 29 years later. J Acoust Soc Am 1990;87(6):2529-2605.

Hallam RS, Jakes SC, Hinchcliffe R: Cognitive variables in tinnitus annoyance. Br J Clin Psychol $1988 ; 27: 213-222$

Hansen MR, Gantz BJ, Dunn C: Outcomes after cochlear implantation for patients with single-sided deafness, including those with recalcitrant Ménière's disease. Otol Neurotol 2013;34:1681-1687.

Hazell JWP, Jastreboff PJ, Meerton LE, Conway MJ: Electrical tinnitus suppression: frequency dependence of effects. Int J Audiol 1993;32:68-77.

Hoare DJ, Edmondson-Jones M, Sereda M, Akeroyd MA, Hall D: Amplification with hearing aids for patients with tinnitus and co-existing hearing loss. Cochrane Database of Systematic Reviews DOI: 10.1002/14651858.CD010151.pub2.

Hou J-H, Zhao S-P, Ning F, Rao S-Q, Han D-Y: Postoperative complications in patients with cochlea implants and implact of nursing intervention. Acta Oto-Laryngol 2010;130:687-695.

House D: Tinnitus suppression via cochlear implants: review and remarks. Int Tinnitus J 1999;5(1):27-29.

House JW, Brackmann DE: Tinnitus: surgical treatment. Ciba Found Symp 1981;85:204-16.

House WF: Cochlear implants. Ann Otol Rhinol Laryngol 1976;85(27):1-93.

Hughes ML, Stille LJ, Baudhuin JL, Goehring JL: ECAP spread of excitation with virtual channels and physical electrodes. Hearing Res 2013;306:93-103.

Ito J, Sakakihara J: Suppression of tinnitus by cochlear implantation. Am J Otolaryngol 1994;15(2):145148
Jastreboff PJ: Tinnitus retraining therapy. Br J Audiol 1999;33(1):68-70.

Kawano A, Seldon HL, Clark GM: Computer-aided three-dimensional reconstruction in human cochlear maps: measurement of the lengths of organ of Corti, outer wall, inner wall, and Rosenthal's canal. Ann Otol Rhinol Laryngol 1996;105(9):701-709.

Kim HJ, Kim DY, Kim HI, Oh HS, Sim NS, Moon IS Long-term effects of repetitive transcranial magnetic stimulation in unilateral tinnitus. Laryngoscope 2014;124(9):2155-60.

Kleine Punte A, Vermeire K, Hofkens A, De Bodt M, De Ridder D, Van de Heyning P Cochlear implantation as a durable tinnitus treatment in single-sided deafness. Cochlear Implants Int 2011;12(1):S26-9.

Kleinjung T, Steffens T, Strutz J, Langguth B: Curing tinnitus with a cochlear implant in a patient with unilateral sudden deafness: a case report. Cases J 2009;2:7462.

Langguth B, Kreuzer PM, Kleinjung T, De Ridder D: Tinnitus: cause and clinical management. Lancet Neurol 2013;12:920-30.

Leek MR: Adaptive procedure in psychophysical research. Perception \& Psychophysics 2001;63(8):12791292.

Meeus O, Blaivie C, Van de Heyning P: Validation of the Dutch and the French version of the Tinnitus Questionnaire. B-ENT 2007;3(Suppl 7):11-17.

Mendes Rde C, Ribas A, De Macedo IP, Buschle M, Ataíde AL, Pereira R, Kochen AP: Cochlear implant in the treatment of incapacitating unilateral tinnitus: case report. Int Tinnitus J 2012;17(2):200-4.

Mielczarek M, Konopka W, Olszewski J: The application of direct current electrical stimulation of the ear and cervical spine kinesitherapy in tinnitus treatment. Auris Nasus Larynx 2013;40:61-65.

Miyamoto RT, Bichey BG: Cochlear implantation for tinnitus suppression. Otolaryngol Clin N Am 2003, 36:345-352.

Modh D, Katarkar A, Alam N, Jain A, Shah P: Relation of distortion product otoacoustic emission and tinnitus in normal hearing patients: A pilot study. Noise Health 2014;16(69):69-72.

Newman CW, Jacobson GP, Spitzer JB: Development of the Tinnitus Handicap Inventory. Arch Otolaryngol Head Neck Surg 1996;122:143-148.

Nondahl DM, Cruickshanks KJ, Huang G-H, Klein BEK, Klein R, Tweed TS, Zhan W: Generationa differences in the reporting of tinnitus. Ear Hear 2012;33:640-644. 
Roberts LE, Husain FT, Eggermont J]: Role of attention in the generation and modulation of tinnitus. Neurosci Biobehav Rev 2013;37(8):1754-73

surgery and outcome in residual hearing and the vestibular system. Int J Audiol 2014;53:121-127.

Nosrati-Zarenoe R, Arlinger S, Hultcrantz E: Idiopathic sudden sensorineural hearing loss: results drawn from the Swedish national database. Acta Otolaryngol 2007:127:1168-1175.

Olze H, Szczepek AJ, Haupt H, Förster U, Zirke N, Gräbel S, Mazurek B: Cochlear implantation has a positive influence on quality of life, tinnitus, and psychological comorbidity. Laryngoscope 2011;121:2220-2227.

Olze H, Szczepek A, Haupt H, Zirke N, Graebel ., Mazurek B: The impact of cochlear implantation on tinnitus, stress and quality of life in postlingually deafened patients. Audiol Neurotol 2012;17:2-11.

Osaki Y, Nishimura H, Takasawa M, Imaizumi M, Kawashima T, Iwaki T, Oku N, Hashikawa K, Doi K, Nishimura T, Hatazawa J, Kubo T: Neural mechanism of residual inhibition of tinnitus in cochlear implant users. NeuroReport 2005;16(15):1625-8.

Palau EM, Gil JLM, Vidal CM, González JCF, Cabrera OA, Macías ÁR: Tinnitus and cochlea implantation. Preliminary experience. Acta Otorrinolaringol Esp 2010;61(6):405-411.

Pantev C, Okamoto H, Teismann H: Music-induced cortical plasticity and lateral inhibition in the human auditory auditory cortex as foundations for tonal tinnitus treatment. Front Syst Neurosc 2012a;6:50.

Pantev C, Okamoto H, Teismann H: Tinnitus: the dark side of auditory cortex plasticity. Ann NY Acad Sci 2012b;1252:253-258.

Postelmans JTF, Cleffken B, Stokroos RJ: Post-operative complications of cochlear implantation in adults and childeren: five years' experience in Maastricht. J Laryngol Otol 2007;121:318-323.

Ramos Á, Polo R, Masgoret E, Artiles O, Lisner I, Zaballos ML, Moreno C, Osoria Á: Cochlear implant in patients with sudden unilateral sensorineural hearing loss and associated tinnitus. Acta Otorrinolaringol Esp 2012;63(1):15-20

Rattay F, Lutter P, Felix H: A model of the electrically excited human cochlear neuron. I. Contribution of neural substructures to the generation and propagation of spikes. Hearing Res 2001;153:43-63.

Reavis KM, Chang JE, Zeng F-G: Patterned sound therapy for the treatment of tinnitus. Hear 2010;63(11):21-24.

Reavis KM, Rothholtz VS, Tang Q, Carroll JA, Djalilian H, Zeng F-G: Temporary suppression of tinnitus by modulated sounds. J Assoc Res Otolaryngol 2012;13(4):561-71.

Reiss LAJ, Turner CW, Karsten SA, Gantz BJ: Plasticity in human pitch perception induced by tonotopically mismatch electro-acoustic stimulation. Neuroscience 2014;256:43-52.
Rosen S: Temporal information in speech: acoustic, auditory and linguistic aspects. Philos Trans R Soc Lond B Biol Sci 1992;336(1273):367-73.

Rubinstein JT, Tyler RS, Johnson A, Brown CJ: Electrical suppression of tinnitus with high-rate pulse trains. Otol Neurotol 2003;24:478-485.

Rubinstein JT, Wilson BS, Finley CC, Abbas PJ: Pseudospontaneous activity: stochastic independence of auditory nerve fibers with electrical stimulation. Hear Res 1999;127:108-118.

Ruckenstein MJ, Hedgepeth C, Rafter KO, Montes ML, Bigelow DC: Tinnitus suppression in patients with cochlear implants. Otol Neurotol 2001;22:200-204.

Saltzman M, Ersner M: A hearing aid for the relief of tinnitus aurium. Laryngoscope 1947;57:358-66.

Sanchez TG, Ferrari GMS: The control of tinnitus through hearing aids: suggestions for optimal use control do zumbido por meio de prótese auditiva: sugestões para otimização do uso]. Pró-Fono Rev d Atualização Cient 2002;14:111-8.

Schaette R: Tinnitus in men, mice (as well as other rodents), and machines. Hear Res 2014;311:63-71.

Schaette R, McAlpine D: Tinnitus with a normal audiogram: physiological evidence for hidden hearing

Schaette R, Turtle C, Munro KJ: Reversible induction of phantom auditory sensation through simulated unilateral hearing loss. PLoS One 2012;7:e35238.

Schatzer R, Vermeire K, Visser D, Krenmayr A, Kals M, Voormolen M, Van de Heyning P, Zierhofer C Electric-acoustic pitch comparisons in single-sided-deaf cochlear implant users: Frequency-place functions and rate pitch. Hear Res 2014;309:26-35.

Searchfield GD, Kobayashi K, Sanders M: An adaptation level theory of tinnitus audibility. Front Syst Neurosci 2012;6:46.

Shekhawat GS, Searchfield GD, Stinear CM: Role of hearing aids in tinnitus intervention: a scoping review. J Am Acad Audiol 2013;24:747-762.

Shepherd RK, Linahan N, Xu J, Clark GM, Araki S: Chronic electrical stimulation of the auditory nerve using non-charge balanced stimuli. Acta Otolaryngol 1999;119:674-684.

Sridhar D, Stakhovskaya O, Leake PA: A frequency-position function for the human cochlear spiral ganglion. Audiol Neurotol 2006;11(1):16-20.

Stakhovskaya O, Sridhar D, Bonham BH, Leake PA: Frequency map for the human cochlear spiral ganglion: implications for cochlear implants. J Assoc Res Otolaryngol 2007;8:220-233. loss and computational model. J Neurosci 2011;31(38):13452-13457. 
Vermeire $\mathrm{K}$, Nobbe A, Schleich $\mathrm{P}$, Nopp $\mathrm{P}$, Voormolen $\mathrm{MH}$, Van de Heyning $\mathrm{PH}$ : Neural tonotopy in cochlear implants: an evaluation in unilateral cochlear implant patients with unilateral deafness and tinnitus. Hear Res 2008;245:98-106.

Tass PA, Adamchic I, Freund H-J, von Stackelberg T, Hauptmann C: Counteracting tinnitus by acoustic coordinated reset neuromodulation. Restor Neurol Neurosci 2012;30:137-159.

Távora-Vieira D, Marino R, Acharya A, Rajan GP: The impact of cochlear implantation on speech understanding, subjective hearing performance, and tinnitus perception in patients with unilatera severe to profound hearing loss. Otol Neurotol 2015;36(3):430-6.

Távora-Vieira D, Marino R, Krishnaswamy J, Kuthbutheen J, Rajan GP: Cochlear implantation for unilateral deafness with and without tinnitus: a case series. Laryngoscope 2013;123(5):1251-5.

Todt I, Rademacher G, Mutze S, Ernst A: Scalar electrode position and the occurrence of tinnitus in cochlear implantees. XI International Tinnitus Seminar, Berlin 2014

Tokita J, Dunn C, Hansen MR: Cochlear implantation and single-sided deafness. Curr Opin Otolaryngol Head Neck Surg 2014;22(5):353-8.

Turner O, Windfuhr K, Kapur N: Suicide in deaf populations: a literature review. Ann Gen Psychiatry 2007;6:26.

Tyler RS, Rubinstein J, Pan T, Chang S-A, Gogel SA, Gehringer A, Coelho C: Electrical stimulation of the cochlea to reduce tinnitus. Semin Hear 2008;29(4):326-332.

Undurraga JA, Carlyon RP, Macherey O, Wouters J, Van Wieringen A: Spread of excitation varies for different electrical pulse shapes and stimulation modes in cochlear implants. Hearing Res 2012;290: 21-36.

Undurraga JA, Carlyon RP, Wouters J, van Wieringen A: The polarity sensitivity of the electrically stimulated human auditory nerve measured at the level of the brainstem. J Assoc Res Otolaryngol 2013;14:359-377.

Undurraga JA, van Wieringen A, Carlyon RP, Macherey O, Wouters J: Polarity effects on neural responses of the electrically stimulated auditory nerve at different cochlear sites. Hearing Res 2010;269:146-161.

Van de Heyning P, Vermeire K, Diebl M, Nopp P, Anderson I, De Ridder D: Incapacitating unilateral tinnitus in single-sided deafness treated by cochlear implantation. Ann Oto Rhinol Laryn 2008;117(9):645-652.

van Zon A, Peters JPM, Stegeman I, Smit AL, Grolman W: Cochlear implantation for patients with single-sided deafness or asymmetrical hearing loss: a systematic review of the evidence. Otol Neurotol 2015;36:209-219.

Vermeire K, Lawson DT: Electric and acoustic dynamic range and loudness growth functions: a withinsubject comparison in cochlear implant patients. Journal of hearing science 2012;2(4):18-25.
Vernon JA, Meikle MB: Masking devices and alprazolam treatment for tinnitus. Otolaryngol Clin N Am 2003;36:307-320

Vlastarakos PV, Nazos K, Tavoulari E-F, Nikolopoulos TP: Cochlear implantation for single-sided deafness: the outcomes. An evidence-based approach. Eur Arch Otorhinolaryngol 2014;271(8):211926 .

Wilson BS, Dorman MF: Cochlear implants: a remarkable past and a brilliant future. Hearing Res 2008;242(1-2):3-21.

Zeman F, Koller M, Schecklmann M, Langguth B, Landgrebe M: Tinnitus assessment by means of the Tinnitus Handicap Inventory (THI), and their short versions in an international and multi-lingual sample. Health and Quality of Life Outcomes DOI: 10.1186/1477-7525-10-128.

Zeng F-G, Tang Q, Dimitrijevic A, Starr A, Larky J, Blevins NH: Tinnitus suppression by low-rate electric stimulation and its electrophysiological mechanisms. Hear Res 2011;277:61-66. standardized self-report questionnaires: Psychometric properties of the Tinnitus Questionnaire (TQ), 


\section{Chapter}

Tinnitus suppression by intracochlear electrical stimulation in Single Sided Deafness a prospective clinical trial: Part II

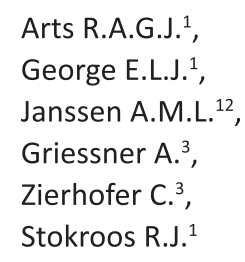

Zierhofer C. ${ }^{3}$,

Stokroos R.J.

${ }^{1}$ Department of ENT/Head and Neck Surgery,

Maastricht University Medical Center

MHeNS School for Mental Health and Neuroscience,

PO Box 5800, 6202 AZ Maastricht, The Netherlands.

2Department of Methodology and Statistics,

Maastricht University; CAPHRI School for

Public Health and Primary Care, P. Debeyeplein 1,

6229 HA Maastricht, The Netherlands.

${ }^{3}$ Institute of Mechatronics, University of Innsbruck,

Technikerstraße 13, 6020 Innsbruck, Austria.

Submitted for publication

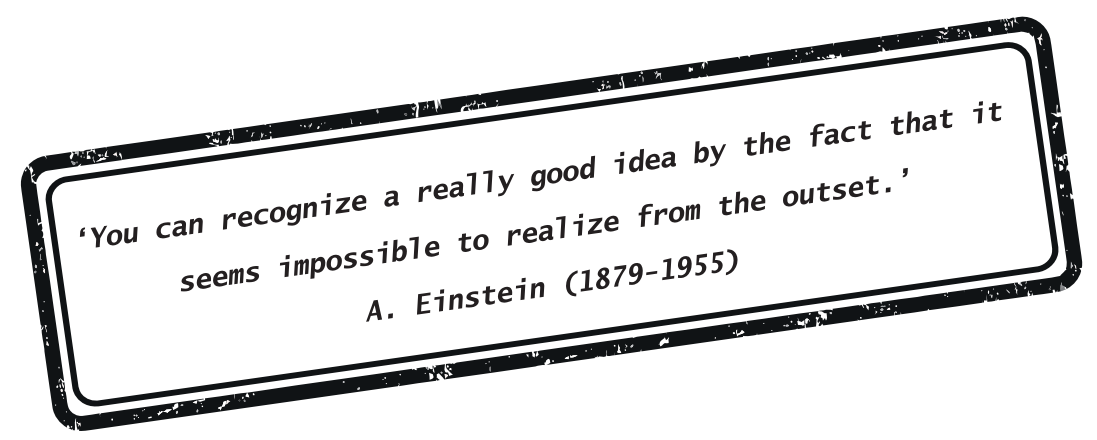


Abstract:

Earlier studies show that a Cochlear Implant (Cl), capable of providing intracochlear electrical stimulation independent of environmental sounds, appears to suppress tinnitus at least short term. The current main objective is to compare the long-term suppressive effects of looped electrical stimulation (without environmental sound perception) with the standard stimulation pattern of a $\mathrm{Cl}$ (with environmental sound perception). This could open new possibilities for the development of a "Tinnitus Implant" (TI), an intracochlear pulse generator for the suppression of tinnitus.

Ten patients with Single Sided Deafness (SSD) suffering from unilateral tinnitus in the deaf ear are fitted with a CI (MED-EL Corporation, Innsbruck, Austria). Stimulation patterns are optimized for each individua patient, after which they are compared using an AB/BA randomized crossover design, with a follow-up of six months, followed by a 3 month period using the modality of patient's choice.

Results show that tinnitus can be suppressed with intracochlear electrical stimulation independent of environmental sounds, even long-term. No significant difference in tinnitus suppression was found between the standard clinical $\mathrm{Cl}$ and the $\mathrm{TI}$.

It can be concluded that coding of environmental sounds is no requirement for tinnitus suppression with intracochlear electrical stimulation. It is therefore plausible that tinnitus suppression by $\mathrm{Cl}$ is not solely caused by an attention shift from the tinnitus to environmental sounds. Both the standard clinical $\mathrm{Cl}$ and the experimental $\mathrm{Tl}$ are potential treatment options for tinnitus. These findings offer perspectives for a successful clinical application of the TI, possibly even in patients with significant residual hearing.

\section{Introduction:}

Tinnitus aurium, meaning ringing of the ears is the phantom sensation of sound. It is a frequent symptom of hearing dysfunction, affecting about 50 million people in the United States and an estimated 70 million in the European Union [Cederroth et al., 2013]. For some of them it can be extremely burdensome and affect daily life. Furthermore, the economic burden of tinnitus to society is substantial with an annual tinnitus related health care cost per patient of $€ 1,544$ on average in the Netherlands [Maes et al., 2013] The exact underlying mechanism is not completely known, but it is plausible that tinnitus has a central origin that is triggered by auditory deprivation as a maladaptive homeostatic compensation mechanism [Schaette and McAlpine, 2011]. It has long been known that auditory deprivation can induce phantom sounds when subjects spend time in complete silence in a sound-proof booth [Heller and Bergman, 1953]. More recently, it has been reported that continuous use of an earplug can also lead to the perception of tinnitus [Schaette et al., 2012].

Due to plasticity, reversing auditory deprivation by electrical stimulation should suppress the tinnitus theoretically. Although intracochlear electrical stimulation seems to be a potential treatment option for tinnitus there is no cure available yet. Research in the past decade show tinnitus suppression in bilatera and unilateral deafness using Cochlear Implants (Cls) [Van de Heyning et al., 2008; Vallés-Varela et al. 2013]. It is not yet clear if the processing of speech, that is, the perception of environmental sounds is a requirement of these observed effects. The question arises if it is possible that similar effects on tinnitus, or even optimization of these effects may be achieved by meaningless, but highly controlled, intracochlear electrical stimulation. Previous studies, including Part I of this study, show short-term tinnitus reduction using electrical stimulation that does not encode environmental sounds [Chang and Zeng, 2012; Arts et al., 2015a; Arts et al., 2015b]. In order to determine whether meaningless chronic intracochlear electrical stimulation is a viable treatment option for people with extremely burdensome tinnitus, long-term effects need to be investigated. The primary goal of this study is to investigate the long-term effects of intracochlear electrical stimulation, that does not encode environmental sounds, on tinnitus and compare these effects with the effects obtained using standard clinical Cl.

\section{Materials and Methods:}

This study is a continuation of the previously published Part I. For a detailed description of the materials and methods used, see this previously published Part I [Arts et al., 2015b]. A concise but adequate description follows below.

\section{Subjects}

Ten adults with Single Sided Deafness (SSD) were included with an audiometric hearing threshold of at least $70 \mathrm{~dB} \mathrm{HL}$ Pure Tone Average (PTA); averaged across $0.5,1$ and $2 \mathrm{kHz}$ in one ear. Their contralateral ear had a moderate to normal audiometric hearing threshold (PTA better than $50 \mathrm{~dB} \mathrm{HL}$ ). In Part I of this study they received a cochlear implant $(\mathrm{Cl})$ in the deaf ear in order to suppress their unilateral tinnitus, localized in the deaf ear. Inclusion criteria for implantation were: chronic, continuous and moderate-to-severe tinnitus that was stable for at least one year. Moderate-to-severe tinnitus was diagnosed as a tinnitus loudness of at least 7.0 on a Visual Analogue Scale (VAS) with a range from 0 to 10, a Tinnitus Handicap Inventory (THI) [Newman et al., 1996] score of at least 38 and/or a Tinnitus Questionnaire (TQ) [Meeus et al., 2007] score of at least 42. Exclusion criteria were medical contraindications for cochlear implantation, diagnosed objective tinnitus, psychiatric disorders, depression and use of antidepressant medication. 


\section{Device and software}

In Part I of this study subjects were implanted with a MED-EL cochlear implant system (MED-EL Corporation, Innsbruck, Austria), consisting of a CONCERTO implant and the OPUS2 processor. The OPUS2 was fitted as a speech processor (CI) with standard clinical software, Maestro version 4.1.2 and 6.0 using MED-EL's Diagnostic Interface Box II or MAX programming interface while the OPUS2 was programmed as a pattern generator (TI) using customized software (Inst. of Mechatronics, Innsbruck, Austria) in Matlab version 7.11.0 (R2010b) (The Mathworks, Natick, MA, USA) using MED-EL's Diagnostic Interface Box II.

\section{Outcome measurements}

\section{Tinnitus}

A VAS method, psychoacoustic measurements (pitch and loudness matching), duration of Residual Inhibition (RI) and questionnaires (THI and TQ) were used as tinnitus specific outcome measures.

For the VAS method, the subjects marked how they perceived tinnitus loudness, amount of discomfort, effect on life and extent of problems due to the tinnitus, each on a $10 \mathrm{~cm}$ bar [Buechner et al., 2010] Values were accurate to one decimal place.

Tinnitus pitch and loudness were acoustically measured, using headphones (Telephonics, TDH-39P), via the contralateral ear. Subjects were asked to concentrate on the predominant pitch of their tinnitus. Tinnitus pitch matching (PM) was performed, after one week of sham stimulation, prior to the crossover design and both after one and three months of standard $\mathrm{Cl}$ and TI. Furthermore, tinnitus PM was performed after one and three months during the implant use of choice following the crossover design. Pure tones, $1 / 3$ octave narrow band noises and warble tones within the $250-8000 \mathrm{~Hz}$ range (for the center frequency in case of narrow band noises) and white noise were presented using a three-Alternative Forced Choice method. Separate "runs" were applied for either octave and interoctave frequencies from low-to-high frequency and from high-to-low frequency respectively [Arts et al. 2015a].

Tinnitus loudness was acoustically measured in $\mathrm{dB}$ Sensation Level (dB SL). Subjects were asked to indicate whether their tinnitus was louder or softer than the tinnitus pitch matched stimulus. A twodown, one-up adaptive staircase rule [Leek, 2001] with step sizes of $5 \mathrm{~dB}$ for rough determination and 1 $\mathrm{dB}$ for precise determination were used. Starting point was $15 \mathrm{~dB}$ SL. Tinnitus Loudness Matching (TLM) was performed, after one week of sham stimulation, prior to the crossover design and both after one and three months of standard $\mathrm{Cl}$ and TI. Finally, TLM was performed after one and three months during implant use of choice following the crossover design. possible placebo effects. Here, a sub-threshold stimulation level of less than $0.5 \mathrm{nC}$ per electrode was used to avoid warning signals of the processor and remote control (single-blinded procedure). Subjects were informed that the stimulation applied was sub-threshold and therefore inaudible, but that the current level was at a level at which significant tinnitus suppression was plausible. Subsequently, for each subject the four most efficient stimulation patterns during the fine tuning procedure were used on a daily basis (for details, see Arts et al., 2015b). Subjects were asked to rank their tinnitus loudness and stimulus comfort at the end of each day. Each of the four stimulation patterns were tested for two full days to choose the single most convenient pattern, which was consequently utilized during the randomized crossover design. Here, either the processor was first programmed for three months as a standard clinical $\mathrm{Cl}$ in order to perceive environmental sounds after which the processor was switched for three months to function as a looped pattern generator (TI) or vice versa. Finally, subjects were allowed to choose, based on their experiences, to use their processor either as a speech processor or as a pattern generator for another three-month follow-up period. At the end of the trial, it was allowed to fit the processor with both modalities, each programmed in a different program bank.
In case of subjective tinnitus suppression by intracochlear electrical stimulation the RI was measured. $R$ is the length of persistence in the reduction of tinnitus after the electrical stimulation was stopped [Osak et al., 2005].

The degree of handicap due to the perceived tinnitus was evaluated with the internationally validated TH after one week of sham stimulation, prior to the crossover design and both after one and three months of standard $\mathrm{Cl}$ and $\mathrm{TI}$. Furthermore, the THI was filled out after one and three months during use of choice following the crossover design. The THI quantifies the effect of tinnitus on the patient's emotions and daily activities using 25 items, each answered with No ( 0 points), Sometimes ( 2 points) or Yes ( 4 points). A higher score indicates a more severe handicap [Newman et al., 1996; Zeman et al., 2012]. 
Effectiveness of intracochlear electrical stimulation on tinnitus suppression

Tinnitus distress was evaluated using the Dutch validated TQ [Hallam et al., 1988; Meeus et al., 2007] after one week of sham stimulation, prior to the crossover design and both after one and three months of standard $\mathrm{Cl}$ and $\mathrm{TI}$. The TQ was also filled out after one and three months during implant use of choice following the crossover design. The questionnaire consists of 52 questions, with a maximum score of 84 . Higher scores indicate more severe levels of distress.

\section{Quality of Life}

The Health Utilities Index Mark III (HUI mark III) was used to estimate health-related quality of life [Feeny et al., 2007; Maes et al., 2011]. The HUI mark III consists of 17 questions of which 12 questions are used to compute eight dimensions: vision, hearing, speech, emotion, pain/complaints, ambulation, dexterity and cognition. Possible overall utility scores range from -0.36 (the all-worst health state) to 0.00 (dead) to 1.00 (perfect health). The single-attribute utility score for the hearing dimension was obtained from question 3 and 4 and varies between 0.00 and 1.00. The HUI mark III was filled out prior to the Cl-surgery, after three months of standard $\mathrm{Cl}$ and $\mathrm{Tl}$ and after three months during implant use of choice following the crossover design.

\section{Depression}

To measure the behavioral manifestation of depression the Beck Depression Inventory (BDI) was used [Beck et al., 1961; Bouman et al., 1985]. The BDI is a 21-item questionnaire that explicitly stresses the aspects of depression. Each item can be scored from 0 to 3, with a total score of 0-13 for minimal depression, 14-19 for light depression, 20-28 corresponds to moderately serious depression, and finally, a score of 29 or more corresponds to serious depression. The BDI was filled out prior to the $\mathrm{Cl}$-surgery, after three months of standard $\mathrm{Cl}$ and $\mathrm{Tl}$ and after three months during the implant use of choice following the crossover design.

\section{Speech perception}

Speech perception in silence, speech perception in noise and results from the Speech, Spatial and Quality of hearing questionnaires will be reported elsewhere.

\section{Statistics}

Statistics were performed with IBM SPSS Statistics, version 20, and $p$ values smaller than 0.05 were, unless otherwise reported, considered statistically significant.

\section{Crossover design}

In order to assess the effectiveness of the TI on tinnitus, the outcomes obtained during TI were compared to the outcomes obtained during standard $\mathrm{Cl}$. Two-sided Mann-Whitney $\mathrm{U}$ tests (Exact) were performed to determine possible carryover effects, period effects and treatment effects. A carryover effect in a crossover design occurs when the effects of one or both interventions during the first active period have a residual biological effect during the second active period. Testing for possible carryover effects is important because no washout period was used in the current methodological design. The power of the test to detect carryover effects is limited. Therefore, $p$ values smaller than 0.10 were considered statistically significant [Grizzle, 1965]. Period effects show a clear preference for the former or latter period and could bias treatment effects. Treatment effects were studied to determine the effectiveness of the $\mathrm{Tl}$ in relation the $\mathrm{Cl}$ treatment were observed.
Using this methodological design, the current study was able to assess both a possible placebo effect as well as the effect of intracochlear electrical stimulation on tinnitus. Non-parametric Friedman tests for multiple comparisons were performed prior to post hoc testing with two-sided Wilcoxon signed-rank tests. A statistically significant Friedman test allows us to perform post hoc testing. In order to assess the placebo effect, baseline outcomes (before implantation) were compared to the outcomes after one week of sham stimulation. The effect of intracochlear electrical stimulation on tinnitus was assessed by comparing baseline outcomes to the outcomes obtained at the end of the follow-up.

\section{Ethics}

This study was designed and conducted in accordance with the declaration of Helsinki. Ethics approval was obtained from the Ethics Committee of Maastricht University/academic hospital Maastricht (approval No. NL38789.068.11). An independent Data Safety Monitoring Board was used to oversee the safety of the included subjects. All subjects gave written informed consent before participation.

\section{Results:}

During this clinical trial, subject 2 struggled as she adjusted to the new sound provided by the implant. This was partially due to her noisy and stressful daily environment which included taking care of her two young children and her mother who passed away during the trial. Subject 8 had a stressful period with her partner and child which made wearing her hearing prosthesis complicated. Nevertheless, none of the included subjects dropped out prematurely and no serious adverse events that can be related to the

\section{Stimulation patterns}

Following TI optimization of the marked potential stimulation patterns in Part I [Arts et al., 2015b] on a daily basis, Table 1 shows the stimulation patterns, one for each subject, which were subjectively scored as the most convenient stimuli. This pattern was used during TI-stimulation in the current longitudina study. In eight out of the ten included subjects the electrical stimulation was presented on the tinnitus pitch matched electrode. In five of them the stimulation was presented at this single electrode, in one subject the stimulation was presented on the tinnitus pitch matched electrode together with the two adjacent electrodes and in the other two subjects the stimulation was presented on all the available electrodes. Furthermore, eight out of ten subjects preferred a cathodic first charge-balanced biphasic stimulation. During the crossover design it was allowed to reduce the applied current level of the looped stimulation patterns by consultation of our tertiary otologic practice in case of for example tinnitus reduction or in order to improve stimulus comfort. 
Table 2: Differences between $\mathrm{Tl}$ and $\mathrm{Cl}$, investigated in a crossover design. mode.

\begin{tabular}{|c|c|c|c|c|c|c|c|}
\hline Subject & Pattern & Electrode(s) & $\begin{array}{l}\text { Amplitude } \\
\text { Modulation }\end{array}$ & $\begin{array}{l}\text { Polarity } \\
\text { (first A/C) }\end{array}$ & $\begin{array}{l}\text { Stimulation } \\
\text { rate (pps/ } \\
\text { channel) }\end{array}$ & $\begin{array}{l}\text { Mean pulse } \\
\text { width/ } \\
\text { channel ( } \mu \mathrm{s})\end{array}$ & $\begin{array}{l}\text { Final mean } \\
\text { maximum } \\
\text { charge value/ } \\
\text { channel (nC) }\end{array}$ \\
\hline 1 & 5 & 10 (PM elec.) & Fixed & C & 200 & 69 & 12.1 \\
\hline $1^{*}$ & 41 & 10 (PM elec.) & Random & c & 5000 & 85 & 9.1 \\
\hline 2 & App. 2 & $1 \& 2$ & Fixed & c & 200 & 60 & 3.2 \\
\hline 3 & 28 & All & Random & c & 750 & 79 & 5.5 \\
\hline 4 & 48 & 9 (PM elec.) & Sine wave & c & PM & 74 & 3.3 \\
\hline $5^{\ddagger}$ & 40 & $\begin{array}{l}8 \& 9 \text { (two } \\
\text { adjacent elec. } \\
\text { of PM elec.) }\end{array}$ & Random & c & $4918^{\circ}$ & 80 & 6.1 \\
\hline 6 & 47 & 11 (PM elec.) & Sine wave & C & 5000 & 62 & 6.6 \\
\hline 7 & 13 & 6 (PM elec.) & Fixed & c & 200 & 69 & 10.4 \\
\hline 8 & 27 & 7 (PM elec.) & Random & c & 750 & 65 & 8.0 \\
\hline 9 & App. 2 & $\begin{array}{l}\text { 7-9 (PM elec. + two } \\
\text { adjacent elec.) }\end{array}$ & Random & A & PM & 84 & 7.1 \\
\hline 10 & App. 2 & All & Fixed & A & 750 & 88 & 2.5 \\
\hline
\end{tabular}

A: anodic, App.: appendix; C: cathodic, *: change of stimulation pattern after 1 month due to suboptimal effect on tinnitus, ${ }^{\neq}:$partial insertion with two extracochlear electrodes, $\square:$ limited due to the overall stimulation rate. The most convenient stimulation patterns were obtained from Part I [Arts et al., 2015b]. Furthermore, results obtained from subject 4 were additionally presented as a case report [Arts et al., in press].

\section{Crossover design}

The included subjects were equally distributed in a randomized order between the two treatment arms, consisting of standard $\mathrm{Cl}$ followed by $\mathrm{Tl}$ or vice versa. Table 2 shows the results obtained during the crossover design. Here, descriptive statistics include mean score, standard deviation (SD), median and interquartile range (IQR) of both $\mathrm{TI}$ and $\mathrm{Cl}$. Results were obtained after one month and three months of treatment. No carryover effects were obtained. A statistically significant period effect was found only for the BDI-score after three months of treatment in which the depression-score in period 1 was higher compared to period 2, regardless of the treatment arm. No significant treatment effects were observed for the tested variables after both one month and three months of treatment.

\begin{tabular}{|c|c|c|c|c|c|c|}
\hline & \multicolumn{2}{|c|}{ Implant function } & \multirow{2}{*}{ 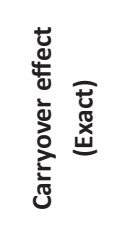 } & \multirow{2}{*}{ 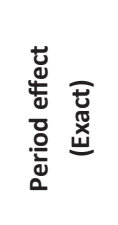 } & \multirow{2}{*}{ 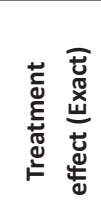 } \\
\hline & & $\mathrm{N}=10$ & $\mathrm{Cl} \quad \mathrm{N}=10$ & & & \\
\hline \multicolumn{7}{|l|}{1 month treatment } \\
\hline \multirow{3}{*}{ Averaged VAS-score } & Mean & 4.20 & 3.69 & \multirow{3}{*}{$p=0.548$} & \multirow{3}{*}{$p=0.167$} & \multirow{3}{*}{$p=0.246$} \\
\hline & SD & 2.41 & 2.25 & & & \\
\hline & Median (IQR) & $3.35(2.68-6.95)$ & $3.15(2.00-5.80)$ & & & \\
\hline \multirow{3}{*}{ TLM } & Mean & 13.30 & 12.90 & \multirow{3}{*}{$p=0.730$} & \multirow{3}{*}{$p=0.397$} & \multirow{3}{*}{$p=0.881$} \\
\hline & SD & 13.70 & 9.53 & & & \\
\hline & Median (IQR) & $7.50(4.75-20.00)$ & $12.00(5.75-18.00)$ & & & \\
\hline \multirow{3}{*}{ TQ } & Mean & 29.60 & 29.70 & \multirow{3}{*}{$p=0.841$} & \multirow{3}{*}{$p=0.175$} & \multirow{3}{*}{$p=0.770$} \\
\hline & SD & 13.06 & 11.84 & & & \\
\hline & Median (IQR) & $30.00(19.25-38.25)$ & $27.00(23.50-38.50)$ & & & \\
\hline \multirow{3}{*}{ THI } & Mean & 38.40 & 34.80 & \multirow{3}{*}{$p>0.999$} & \multirow{3}{*}{$p=0.802$} & \multirow{3}{*}{$p=0.056$} \\
\hline & SD & 15.77 & 14.88 & & & \\
\hline & Median (IQR) & $40.00(25.00-44.50)$ & $38.00(21.50-44.50)$ & & & \\
\hline \multicolumn{7}{|c|}{3 months treatment } \\
\hline \multirow{3}{*}{ Averaged VAS-score } & Mean & 4.53 & 3.79 & \multirow{3}{*}{$p=0.968$} & \multirow{3}{*}{$p=0.500$} & \\
\hline & SD & 2.80 & 2.58 & & & $p=0.389$ \\
\hline & Median (IQR) & $3.40(2.40-7.63)$ & $3.50(1.55-6.63)$ & & & \\
\hline & Mean & 12.50 & 13.00 & & & \\
\hline TLM & SD & 10.34 & 8.84 & $p>0.999$ & $p=0.683$ & $p=0.857$ \\
\hline & Median (IQR) & $9.50(3.75-20.25)$ & $11.00(7.00-17.75)$ & & & \\
\hline & Mean & 32.10 & 28.30 & & & \\
\hline TQ & SD & 12.81 & 16.63 & $p=0.595$ & $p=0.389$ & $p=0.183$ \\
\hline & Median (IQR) & $30.00(22.50-34.75)$ & $23.50(13.75-43.25)$ & & & \\
\hline & Mean & 40.40 & 35.00 & & & \\
\hline THI & SD & 16.49 & 14.52 & $p=0.524$ & $p=0.730$ & $p=0.151$ \\
\hline & Median (IQR) & $40.00(25.00-52.00)$ & $31.00(22.00-46.50)$ & & & \\
\hline & Mean & 6.70 & 6.10 & & $p=0.024$ & \\
\hline BDI & SD & 4.08 & 2.92 & $p=0.889$ & (period & $p=0.333$ \\
\hline & Median (IQR) & $7.50(3.75-10.00)$ & $6.00(4.75-7.50)$ & & 1>period2) & \\
\hline & Mean & 0.715 & 0.698 & & & \\
\hline HUI Mark III & SD & 0.210 & 0.203 & $p=0.738$ & $p=0.206$ & $p=0.738$ \\
\hline & Median (IQR) & $0.745(0.625-0.865)$ & $0.720(0.535-0.865)$ & & & \\
\hline & Mean & 0.801 & 0.773 & & & \\
\hline HUI Mark III & SD & 0.240 & 0.221 & $p=0.738$ & $p=0.397$ & $p>0.999$ \\
\hline hearing & Median (IQR) & $0.930(0.480-1.000)$ & $0.860(0.480-1.000)$ & & & \\
\hline
\end{tabular}


VAS: Visual Analogue Scale, TLM: Tinnitus Loudness Match, TQ: Tinnitus Questionnaire, THI: Tinnitus Handicap Inventory, BDI: Beck Depression Inventory, HUI: Health Utilities Index, SD: standard deviation, IQR: interquartile range, $\mathrm{Tl}$ : tinnitus implant, Cl: cochlear implant.

\section{Treatment effect of intracochlear electrical stimulation}

Although no statistically significant differences on tinnitus related outcomes, depression and quality-of life between $\mathrm{Tl}$ and $\mathrm{Cl}$ could be found (Table 2), subject 9 was the only one who chose for $\mathrm{TI}$ following the crossover design. All other subjects preferred $\mathrm{Cl}$, probably because of the audiological advantages compared to TI which will be reported elsewhere. For consistency, the current report presents the same tinnitus related outcome measures as in Part l of this study [Arts et al., 2015b].

Figure 2 shows the averaged VAS-scores of perceived tinnitus loudness, amount of discomfort, effect on life and extent of problems due to the tinnitus prior to surgery, after one week of sham stimulation and at the end of the follow-up, i.e., after three months of implant use of choice. The Friedman test was statistically significant $(p=0.001)$. Post-hoc tests show no significant difference in averaged VAS-score between baseline and sham stimulation $(p=0.156)$. A statistically significant reduction of the averaged VAS-score was observed at the end of the follow-up compared to baseline $(p=0.002)$.

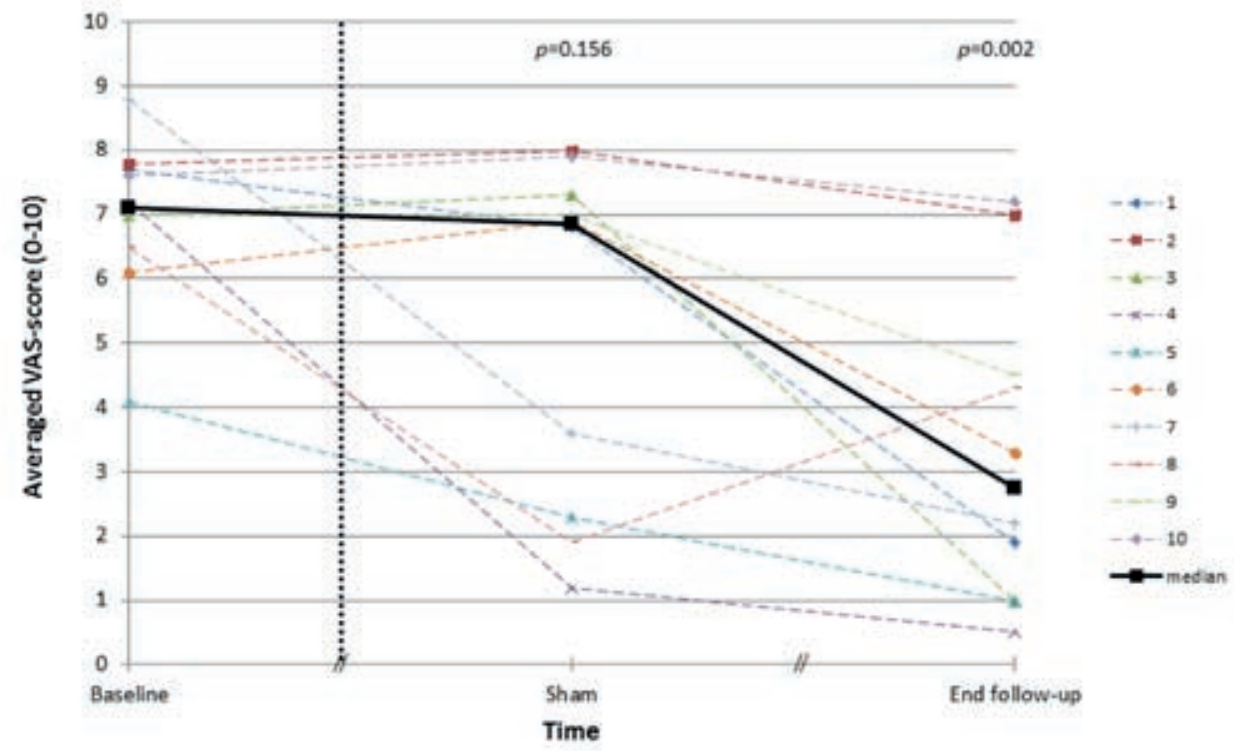

Fig.2: The averaged score on a Visual Analogue Scale (0-10) on perceived tinnitus loudness, amount of discomfort, effect on life and extent of problems due to the tinnitus. Individual results and median are shown at baseline, after one week of sham stimulation and at the end of the follow-up.

The tinnitus loudness, matched via acoustic stimulation to the contralateral normal hearing ear, was expressed in dB SL prior to surgery, after one week of sham stimulation and at the end of the follow-up (Figure 3). The Friedman test was statistically significant $(p<0.001)$. Again, no significant difference in innitus loudness was obtained between baseline and sham stimulation $(p=0.750)$ while the difference between baseline and the end of the follow-up was statistically significant ( $p=0.004)$.

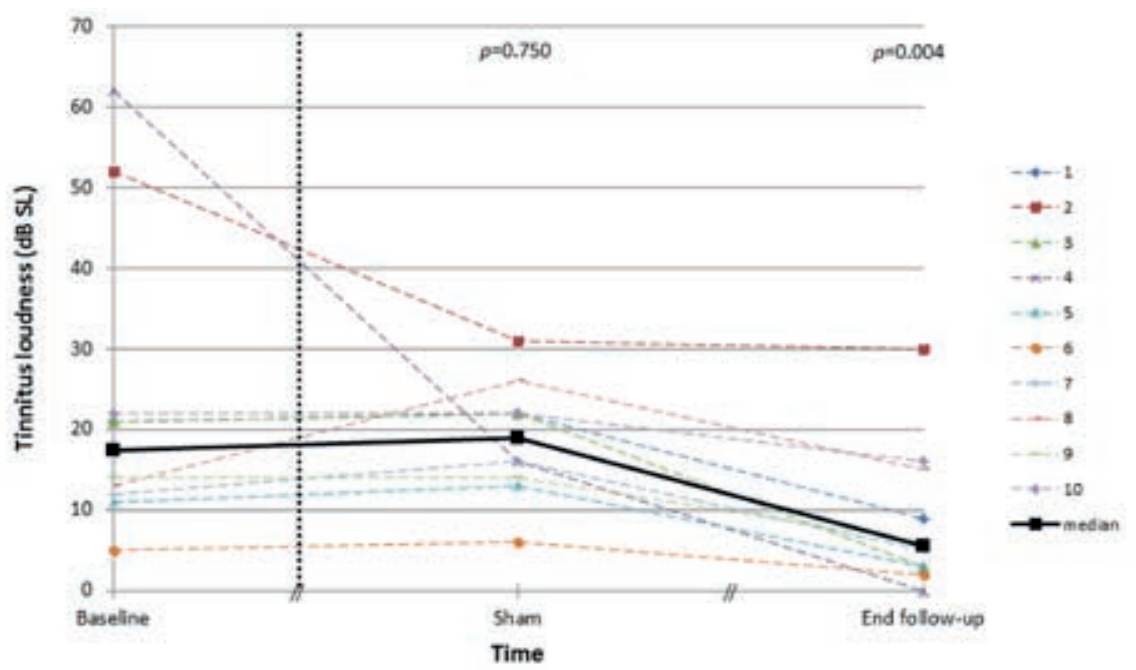

Fig.3: Tinnitus loudness matched via the contralateral normal hearing ear at baseline, after one week of sham stimulation and at the end of the follow-up. Individual results and median are shown.

The total THI and TQ scores prior to surgery, after one week of sham stimulation and at the end of the follow-up are shown in Figure 4. The Friedman tests were statistically significant ( $p=0.018$ and $p=0.026$ respectively). No significant differences in tinnitus handicap (THI) and tinnitus distress (TQ) were obtained between baseline and sham stimulation ( $p=0.203$ and $p=0.338$ respectively). Significant reductions were obtained between baseline and after completing this clinical trial $(p=0.031$ and $p=0.037$ respectively).
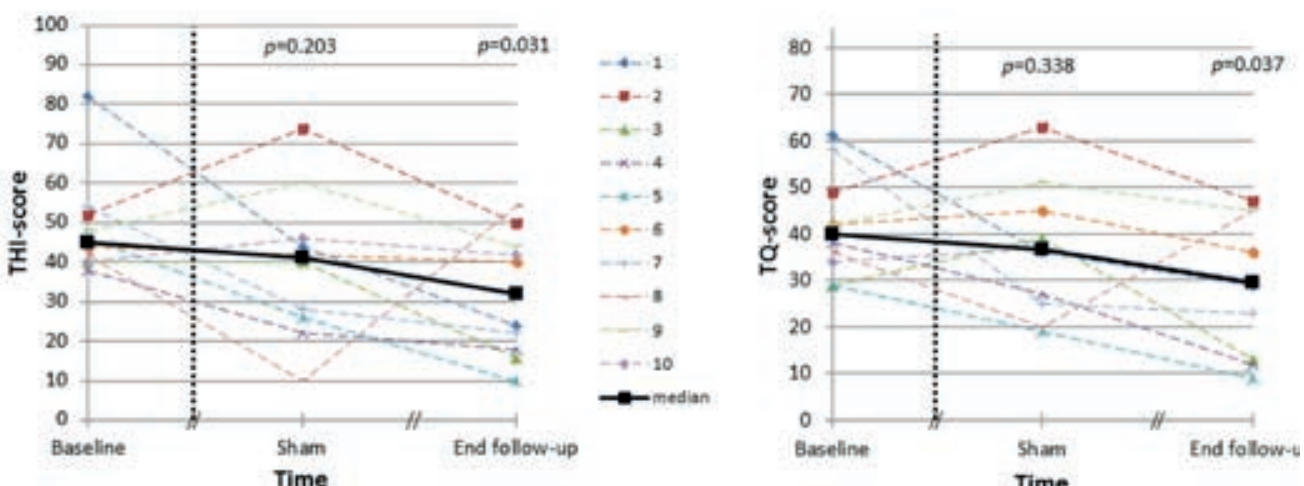

Time

ig.4: Subject-specific results of the Tinnitus Handicap Inventory (left graph) and Tinnitus Questionnaire (right graph) at baseline, after one week of sham stimulation and at the end of the follow-up.

In each subject the RI was measured at least once. Of the 34 times the RI was measured during this trial, in $15 \%$ of the cases the reduction of tinnitus persisted for seconds. For $26 \%$ of the cases the reduction persisted between one and 15 minutes and in another $12 \%$ of the cases the reduction continued between 15 and 30 minutes. The majority of the measured residual inhibitions (47\%) persisted for more than 30 minutes. All six of the residual inhibitions measured in subject 4 persisted for more than 30 minutes while all three of the residual inhibitions measured in subject 5 continued for seconds (Figure 5 ). 
At the end of this clinical trial it was allowed to fit the processor with both standard clinical $\mathrm{Cl}$ and $\mathrm{TI}$, with each modality programmed in a different program bank. Using this combination of implant functions subjects were able to select the preferred modality depending on the daily situation by using their remote control. Six out of the ten included subjects $(60 \%)$ chose for a combination of speech processing $(\mathrm{Cl})$ and looped stimulation (TI) while four subjects (40\%) chose only for speech processing.

\section{Discussion:}

\section{Observations}

Ourstudy shows no statistically significant difference between looped intracochlear electrical stimulation (TI) and standard clinical $\mathrm{Cl}$ on tinnitus outcome measures during the crossover design. There was also no significant difference between treatments found on the BDI and HUI Mark III. However, a period effect was observed for the BDI-scores that could probably bias the analysis of the treatment effect during this crossover design.

Fig.5: Subject-specific Residual Inhibition (left graph), tinnitus pitch (middle graph) and type of tinnitus perception (right graph) during the crossover design and the three-month follow-up period during modality-use of patient's choice. The Y-axis represents the individual subjects. The percentages of the types of tinnitus perceptions were color-coded in the individual pie charts; black for pure tones, grey of narrow band noises and white for white noise

Furthermore, Figure 5 shows the results obtained from tinnitus pitch matching during the crossover design and the three-month follow-up period during modality-use of patient's choice (middle and right graph). For five subjects the tinnitus was perceived in all the measurements as a narrow band noise while the tinnitus perceptions in the other subjects fluctuated over time between pure tones, narrow band noises and white noise. No warble tones were perceived.

Table 3 shows the results obtained from the BDI, the HUI Mark III overall utility score and the HUI Mark III score for the hearing dimension prior to the surgery and at the end of the follow-up. Descriptive statistics include mean score, SD, median and IQR. No statistically significant difference in depression and quality of life, for both the overall utility score as well as the single-attribute utility score for the hearing dimension, could be found between baseline and the end of the follow-up $(p=0.563, p=0.148$ and $p>0.999$ respectively).

Table 3: Depression and utility scores.

\begin{tabular}{|c|c|c|c|c|}
\hline & & Baseline & End follow-up & $\begin{array}{l}\text { Wilcoxon signed-rank } \\
\text { test (Exact) }\end{array}$ \\
\hline \multirow{3}{*}{ BDI } & Mean & 6.90 & 7.20 & \multirow{3}{*}{$p=0.563$} \\
\hline & SD & 1.85 & 6.32 & \\
\hline & Median (IQR) & $7.00(5.00-8.25)$ & $6.00(3.25-10.75)$ & \\
\hline \multirow{3}{*}{$\begin{array}{l}\text { HUI Mark III } \\
\text { overall }\end{array}$} & Mean & 0.809 & 0.727 & \multirow{3}{*}{$p=0.148$} \\
\hline & SD & 0.173 & 0.300 & \\
\hline & Median (IQR) & $0.845(0.738-0.955)$ & $0.725(0.695-0.925)$ & \\
\hline \multirow{3}{*}{$\begin{array}{l}\text { HUI Mark III } \\
\text { hearing }\end{array}$} & Mean & 0.868 & 0.906 & \multirow{3}{*}{$p>0.999$} \\
\hline & SD & 0.212 & 0.164 & \\
\hline & Median (IQR) & $1.000(0.765-1.000)$ & $1.000(0.860-1.000)$ & \\
\hline
\end{tabular}

BDI: Beck Depression Inventory, HUI: Health Utilities Index, SD: standard deviation, IQR: interquartile range.
All used tinnitus outcome measures showed a statistically significant reduction at the end of the followup compared to baseline while no significant difference was found between baseline and after one week of sham stimulation. This treatment did not result in complete tinnitus suppression (except for 1 subject), but the reduction in tinnitus was significant. The BDI-scores and HUI Mark III utility scores at the end of the follow-up were not statistically different from baseline. Furthermore, RI could often be measured during the trial and ranged from a few seconds to more than 30 minutes. The tinnitus was most often perceived as an $1 / 3$ octave narrow band noise. Finally, no serious adverse events that can be related to neither the standard clinical $\mathrm{Cl}$ nor the TI were observed.

\section{Comparison of $\mathrm{Cl}$ and $\mathrm{TI}$}

There is no literature available (with the exception of two case reports, of which one anecdotally [Vernon and Meikle, 2003; Arts et al., in press]) on the long-term effectiveness of intracochlear electrical stimulation independent of environmental sounds on tinnitus. The current study shows no statistically significant difference between looped intracochlear electrical stimulation and standard clinical $\mathrm{Cl}$ in any of the analyzed outcome measures after both one month and three months of treatment in the used crossover design. Therefore, speech perception appears to be no requirement for tinnitus suppression using intracochlear electrical stimulation. However, the results on the depression scale need to be interpreted with caution since a period effect was detected that could influence a possible treatment effect. The preference in the majority of subjects of using the $\mathrm{Cl}$ in relation to the TI during the trial (only 1 subject preferred using the $\mathrm{TI}$ following the crossover design) is plausibly explained by the audiological advantages using the $\mathrm{Cl}$ compared to the $\mathrm{Tl}$.

No statistically significant differences in the health-related quality of life were found both during the crossover design as well as in the comparison between baseline and at the end of the follow-up. Therefore it is assumed that no response shift biased the analyses of the tinnitus outcome measures [Schwartz et al., 2006]. Response shift is the phenomenon that subjects change their internal standards when they experience changes in health.

Effectiveness of intracochlear electrical stimulation on tinnitus suppression

It is generally known that intracochlear electrical stimulation by using standard clinical $\mathrm{Cl}$ suppresse tinnitus in both the bilateral severe to profound deaf population as well as the SSD population [Di Nardo et al., 2007; Bovo et al., 2011; Arts et al., 2012; Olze et al., 2012; Vlastarakos et al., 2014; Blasco and 
Redleaf, 2014; Van Zon et al., 2015]. Our results are consistent with these findings. It is worth mentioning that the significant reduction in the tinnitus loudness matched via the contralateral ear is a subtle addition to the existing literature. Nevertheless, the current study shows no significant improvement of the health-related quality of life after cochlear implantation which is consistent with a previous study using the HUI Mark III after 6 months of $\mathrm{Cl}$ use in eleven adult subjects with unilateral deafness [Arndt et al., 2010]. However, these results are not in agreement with a previous German study using the diseasespecific Nijmegen Cochlear Implant Questionnaire in the bilateral deaf population [Olze et al., 2011]. This inconsistency could probably be explained by the difference in questionnaire used or the difference in the studied population. The HUI Mark III was initially chosen since Maes and colleagues found that this utility measure was preferred in a tinnitus population, compared to the EuroQol-5D [Maes et al., 2011]. A hearing handicap and chronic tinnitus may be associated with emotions such as helplessness and depressive symptoms. Nevertheless, the statistically significant reduction of tinnitus after cochlear implantation did not result in a significant reduction of the depression score. This is consistent with a previous study [Olze et al., 2011] and was initially expected because the absence of depression complaints was one of the criteria for subject inclusion [Arts et al., 2015b]. Moreover, as far as the authors known this is the first study that included a sham stimulation in an attempt to control, although using a single-blinded design, for possible placebo effects.

\section{Variation between subjects}

The heterogeneity of auditory deprivation could declare the considerable variation in effectiveness between subjects. The current consensus is that tinnitus is the result of maladaptive plasticity in the central auditory pathway as a result of auditory deprivation [Arts et al., 2015b]. Therefore, it is plausible that tinnitus could be reversible by restoring auditory stimulation. To be effective it is assumed to be necessary to bypass the cause of auditory deprivation which can occur at various positions along the auditory pathway. As intracochlear electrical stimulation directly stimulates the cochlear nerve and bypasses the transduction at the level of the hair cells, a more central origin of auditory deprivation is presumably outside the range of this treatment option. The suboptimal results obtained in for example subject 2 could therefore possibly be explained by a more central pathology due to the HELLP-syndrome, although no evidence was found for this argument [Arts et al., 2015b]. This assumption is in accordance with the high effectiveness of electrical stimulation on tinnitus found in the subjects with a history of Morbus Menière (subject 3 and 7). Furthermore, both subject 2 and subject 8 were in a stressful perio during the trial which could possibly have hampered the effectiveness on tinnitus suppression. This emphasizes the importance of an appropriate selection procedure.

\section{Limitations}

The study reported was a pilot study, and outcomes could have been victim to potential methodological limits, starting with the small sample size which is mainly the result of the significant costs related to cochlear implantation. It is therefore desirable that alternatives of the relatively expensive standard clinical $\mathrm{Cl}$ will become under investigation. The current study could possibly contribute to investigate these alternatives.

It is also important to note in the interpretation of the results that the test for period effect in the crossover design described above has low sensitivity. Especially for small crossover trials one may fail to detect an interaction even if present.

The current study used a single-blinded placebo controlled procedure and is therefore not completely free from bias. Nevertheless, placebo controlled studies are scarce if not exceptional in intracochlear electrical stimulation for tinnitus suppression while it is expected that the tinnitus population is highly sensitive to possible placebo effects [Dobie, 1999]. Here, a minimal current level was applied to avoid warning signals on both the processor and remote control. This current level was sub-threshold and assumed to be unable to influence neural structures for tinnitus suppression. The current study needs to be interpreted with caution and future studies on the effectiveness of meaningless intracochlear electrical stimulation on tinnitus are highly recommended.

\section{Future perspective}

In the current study, coding of environmental sounds seems to be no requirement for tinnitus suppression using intracochlear electrical stimulation. Therefore, the relatively simple electrical stimulation, without a highly sophisticated speech processing strategy, might be a viable treatment option and could possibly reduce the production costs with respect to the standard clinical $\mathrm{Cl}$. A relatively simple pattern generator is sufficient for this treatment option. Moreover, it might be possible that the effectiveness of intracochlear electrical stimulation on tinnitus suppression could be further optimized using a combination of speech processing and the meaningless, but highly controlled, intracochlear electrical stimulation [Tyler et al., 2015]

The results obtained from looped intracochlear electrical stimulation on tinnitus suppression could possibly be further optimized in future studies. Pre-operative ultra-high field (functional) magnetic resonance imaging of the central nervous system could for example show more accurate information about the optimal stimulation site inside the cochlea compared to the subjective tinnitus pitch matching procedure used in the current study. Furthermore, postoperative imaging using $\mathrm{x}$-rays improves the knowledge about the position of the electrode array and prevent a possible mismatch between the assigned tinnitus pitch-matched electrode based on the default frequency allocation table in the standard clinical software [Landsberger et al., 2015] and the calculated tinnitus pitch-matched electrode based on the available frequency-position functions [Greenwood, 1990; Stakhovskaya et al., 2007].

One might wonder what the profit is of replacing the perception of their own tinnitus by a sound perception induced by the intracochlear electrical stimulation. The sound perception induced by the electrical stimulation applied was experienced as more comfortable than their own tinnitus and often became inaudible over time due to loudness adaptation or a shift in attention [Arts et al., 2015b]. The stimulus comfort was guaranteed by the subject-specific selection on a subjective base of the most convenient stimulation patterns. Moreover, subjects were allowed to reduce the current level of the looped stimulation during the crossover design in case of tinnitus reduction or stimulus discomfort.

Although the current study tested a great range of looped stimulation patterns, it is possible that the effectiveness and comfort could be optimized using other stimulation patterns. For example, although the optimal stimuli for tinnitus suppression appear to be subject-specific (Part I), we concluded that low amplitude electrical stimulation and high rate stimulation resulted in statistically more loudness adaptation compared to high amplitude electrical stimulation and low rate stimulation (manuscript submitted for publication). Loudness adaptation improves stimulus comfort and therefore a combination of optimal tinnitus suppression and loudness adaptation may be preferred.

Furthermore, additional studies will give insight in the factors limiting the effectiveness of intracochlear electrical stimulation on tinnitus. In the current study it seems for example that stress hampered the effectiveness and there are possible indications that this proposed treatment option is suboptimal in case of a more central origin of the auditory deprivation. Future studies are needed for more evidence 
References: be interesting to investigate the effectiveness of looped intracochlear electrical stimulation in subjects with tinnitus complaints and significant residual hearing, in which a standard clinical $\mathrm{Cl}$ would not be expected to have any audiological advantages.

\section{Conclusion:}

In the current placebo-controlled clinical trial a statistically significant tinnitus reduction was observed using intracochlear electrical stimulation. No statistically significant difference was found between looped intracochlear electrical stimulation (TI) and standard clinical CI. Furthermore, no significant effect of either $\mathrm{Cl}$ or TI was found on depression and health-related quality of life. These results show that coding of environmental sounds does not appear to be a requirement for tinnitus suppression in the SSD population. Nevertheless, an adequate selection procedure seems to be essential for the effectiveness of the proposed treatment option. These results need to be interpreted with caution because of several methodological limitations and therefore future research is highly recommended.

\section{Acknowledgement:}

The authors would like to thank Katrien Vermeire MSc Ph.D for her contribution during the developmen of the test protocol and the independent Data Safety Monitoring Board (Jan Wouter Brunings MD, Fons Kessels MD, MSc and Dyon Scheijen MSc) for overseeing the safety of the included subjects. Moreover, the authors are very grateful to the participants for their effort during data collection. This study was financially supported by MED-EL (MED-EL Corporation, Innsbruck, Austria).

\section{Conflict of interest:}

This work was supported by a research grant from MED-EL Corporation, Innsbruck, Austria. The funding source had no involvement in the study design; in the collection, analyses and interpretation of data; in the writing of the report; or in the decision to submit the article for publication. The authors declare that they have no conflict of interest.
Arndt S, Aschendorff A, Laszig R, Beck R, Schild C, Kroeger S, Ihorst G, Wesarg T: Comparison of pseudobinaural hearing to real binaural hearing rehabilitation after cochlear implantation in patients with unilateral deafness and tinnitus. Otol Neurotol 2010;32:39-47.

Arts RA, George EL, Chenault MN, Stokroos RJ: Optimizing intracochlear electrical stimulation to suppress tinnitus. Ear Hear 2015a; 36(1):125-35.

Arts RAGJ, George ELJ, Griessner A, Zierhofer C, Stokroos RJ: Long-term effects of intracochlear electrical stimulation with looped patterns on tinnitus: a case report. ENT J, in press.

Arts RA, George EL, Griessner A, Zierhofer C, Stokroos RJ: Tinnitus suppression by intracochlear electrical stimulation in single sided deafness - a prospective clinical trial: Part I. Audiol Neurotol 2015b; 20:294313

Arts RA, George EL, Stokroos RJ, Vermeire K: Review: Cochlear implants as a treatment of tinnitus in single-sided deafness. Curr Opin Otolaryngol Head Neck Surg 2012;20:398-403.

Blasco MA, Redleaf MI: Cochlear implantation in unilateral sudden deafness improves tinnitus and speech comprehension: meta-analysis and systematic review. Otol Neurotol 2014 [Epub ahead of print].

Bovo R, Ciorba A, Martini A: Tinnitus and cochlear implants. Auris Nasus Larynx 2011; 38(1):14-20. Buechner A, Brendel M, Lesinski-Schiedat A, Wenzel G, Frohne-Buechner C, Jaeger B, Lenarz T: Cochlear implantation in unilateral deaf subjects associated with ipsilateral tinnitus. Otol Neurotol 2010;31:1381 1385.

Cederroth $\mathrm{CR}$, Canlon B, Langguth B: Hearing loss and tinnitus - are funders and industry listening? Nature Biotechnology 2013; 31:972-4

Chang JE, Zeng F-G: Tinnitus suppression by electric stimulation of the auditory nerve. Front Syst Neurosci DOI: 10.3389/2012/00019.

Di Nardo W, Cantore I, Cianfrone F, Melillo P, Scorpecci A, Paludetti G: Tinnitus modifications after cochlear implantation. Eur Arch Otorhinolaryngol 2007;264:1145-1149.

Dobie RA: A review of randomized clinical trials in tinnitus. Laryngoscope 1999; 109: 1202-1211.

Feeny D, Wu L, Eng K: Comparing short form 6D, standard gamble, and Health Utility Index Mark 2 and Mark 3 utility scores: results from total hip arthroplasty patients. Qual Life Res 2004; 13: 1659-1670.

Greenwood DD: A cochlear frequency-position function for several species -29 years later. J Acoust Soc Am 1990; 87(6): 2592-2605

Grizzle JE: The two-period change-over design and its use in clinical trials. Biometrics 1965; 21, 467-480. 
Hansen MR, Gantz B: A series of case studies of tinnitus suppression with mixed background stimuli in a cochlear implant. Am J Audiol 2015; [Epub ahead of print].

222

Heller MF, Bergman M: Tinnitus aurium in normally hearing persons. Ann Otol 1953; 62:73-83.

Landsberger DM, Svrakic M, Roland JT, Svirsky M: The relationship between insertion angles, default frequency allocations, and spiral ganglion place pitch in cochlear implants. Ear Hear 2015; [Epub ahead of print].

Maes IHL, Cima RFF, Vlaeyen JW, Anteunis LJC, Joore MA: Tinnitus: a cost study. Ear Hear 2013;34(4);508514

Maes IHL, Joore MA, Cima RFF, Vlaeyen JW, Anteunis LJC: Assessment of health state in patients with tinnitus: a comparison of the EQ-5D and HUI Mark III. Ear Hear 2011:32:428-435.

Meeus O, Blaivie C, Van de Heyning P: Validation of the Dutch and the French version of the Tinnitus Questionnaire. B-ENT 2007;3(Suppl 7):11-17.

Newman CW, Jacobson GP, Spitzer JB: Development of the Tinnitus Handicap Inventory. Arch Otolaryngol Head Neck Surg 1996;122:143-148.

Olze H, Szczepek AJ, Haupt H, Förster U, Zirke N, Gräbel S, Mazurek B: Cochlear implantation has a positive influence on quality of life, tinnitus, and psychological comorbidity. Laryngoscope 2011;121:2220-2227.

Olze H, Szczepek A, Haupt H, Zirke N, Graebel ., Mazurek B: The impact of cochlear implantation on tinnitus, stress and quality of life in postlingually deafened patients. Audiol Neurotol 2012;17:2-11.

Osaki Y, Nishimura H, Takasawa M, Imaizumi M, Kawashima T, Iwaki T, Oku N, Hashikawa K, Doi K,

Nishimura T, Hatazawa J, Kubo T: Neural mechanism of residual inhibition of tinnitus in cochlear

implant users. Neuroreport 2005;16(15):1625-8.

Schaette R, McAlpine D: Tinnitus with a normal audiogram: physiological evidence for hidden hearing loss and computational model. J Neurosci 2011;41(38):13452-13457.

Schaette R, Turtle C, Munro KJ: Reversible induction of phantom auditory sensation through simulated unilateral hearing loss. PLoS ONE 2012; e35238.

Schwartz CE, Bode R, Repucci N, Becker J, Sprangers MA, Fayers PM: The clinical significance of

adaptation to changing health: a meta-analysis of response shift. Qual Life Res 2006;15:1533-1550. Stakhovskaya O, Sridhar D, Bonham BH, Leake P: Frequency map for the human cochlear spiral ganglion: implications for cochlear implants. JARO 2007; 8: 220-233.

Tyler RS, Keiner AJ, Walker K, Deshpande AK, Witt S, Killian M, Ji H, Patrick J, Dillier N, van Dijk P, Lai WK,
Vallés-Varela H, Royo-López, Carmen-Sampériz L, Sebastián-Cortés JM, Alfonso-Collado I: The cochlear implant as a tinnitus treatment. Acta Otorrinolaringol Esp 2013;64(4):253-257.

Van de Heyning P, Vermeire K, Diebl M, Nopp P, Anderson I, De Ridder D: Incapacitating unilateral tinnitus in single-sided deafness treated by cochlear implantation. Ann Oto Rhinol Laryn 2008;117(9):645-652.

Van Zon A, Peters JPM, Stegeman I, Smit AL, Grolman W: Cochlear implantation for patients with singlesided deafness or asymmetrical hearing loss: a systematic review of the evidence. Otol Neurotol 2015;36:209-219.

Vernon JA, Meikle MB: Masking devices and alprazolam treatment for tinnitus. Otolaryngol Clin N Am 2003;36:307-320.

Vlastarakos PV, Nazos K, Tavoulari E-F, Nikolopoulos TP: Cochlear implantation for single-sided deafness: the outcomes. An evidence-based approach. Eur Arch Otorhinolaryngol 2014;271(8):2119-26. standardized self-report questionnaires: Psychometric properties of the Tinnitus Questionnaire (TQ), the Tinnitus Handicap Inventory (THI), and their short versions in an international and multi-lingual sample. Health and Quality of Life Outcomes DOI: 10.1186/1477-7525-10-128.
Zeman F, Koller M, Schecklmann M, Langguth B, Landgrebe M: Tinnitus assessment by means of 


\section{Chapter}

Hearing after cochlear implantation in patients with unilateral severe to profound hearing loss accompanied with tinnitus and the effect of formal auditory training.

$$
\begin{aligned}
& \text { Arts R.A.G.J. }{ }^{1}, \\
& \text { George E.L.J. }{ }^{1}, \\
& \text { Janssen A.M.L. } \\
& \text { Griessner A. }{ }^{3}{ }^{12} \\
& \text { Zierhofer C. }{ }^{3}, \\
& \text { Stokroos R.J. }{ }^{1}
\end{aligned}
$$

${ }^{1}$ Department of ENT/Head and Neck Surgery,

Maastricht University Medical Center;

MHeNS School for Mental Health and Neuroscience,

PO Box 5800, 6202 AZ Maastricht, The Netherlands.

2Department of Methodology and Statistics,

Maastricht University; CAPHRI School for Public Health

and Primary Care, P. Debeyeplein 1

6229 HA Maastricht, The Netherlands.

3 Institute of Mechatronics, University of Innsbruck,

Technikerstraße 13, 6020 Innsbruck, Austria.

Submitted for publication

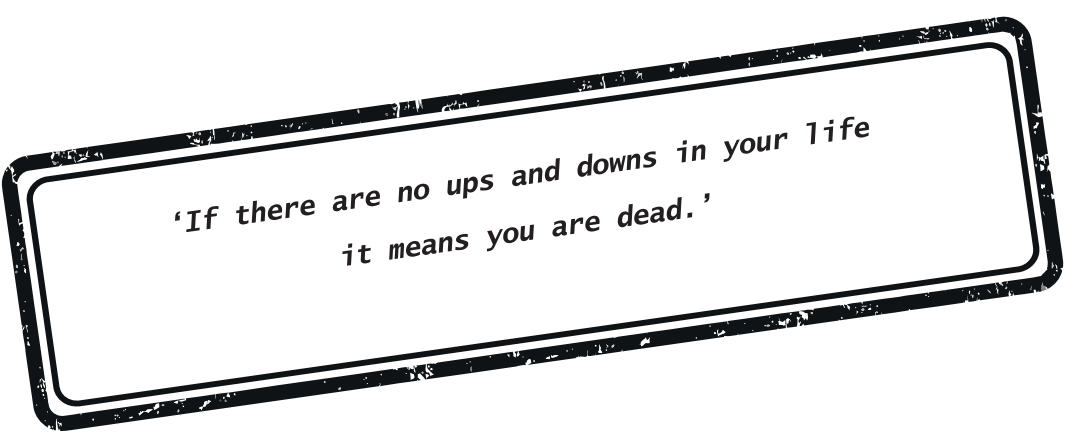




\section{Abstract:}

Previous studies show that intracochlear electrical stimulation independent of environmental sounds appears to suppress tinnitus, even long-term. In order to assess the viability of this potential treatment option it is essential to study the effects of this tinnitus specific electrical stimulation on speech perception. The current research prospectively assesses the effect of standard clinical $\mathrm{Cl}$ on speech perception in Single Sided Deafness (SSD) and the effect of formal auditory training during rehabilitation in ten SSD patients with severe tinnitus complaints. Results show that tinnitus specific electrical stimulation has neither positive nor negative effects on speech perception. Standard clinical $\mathrm{Cl}$ in SSD is shown to be beneficial for speech perception in quiet, speech perception in noise and subjective hearing ability. Furthermore, formal auditory training does not appear to improve speech perception performance. However, Cl-related discomfort reduces significantly more rapidly during $\mathrm{Cl}$ rehabilitation in subjects receiving formal auditory training. In combination with the findings from previous studies on tinnitus suppression using intracochlear electrical stimulation independent of environmental sounds, the results of this study contribute to the viability of cochlear implantation based on tinnitus complaints.

\section{Introduction:}

For decades, a Cochlear Implant (Cl) has become a good treatment option for patients with bilateral severe-to-profound sensorineural hearing loss. Up to 2012, over 300,000 people worldwide have received a $\mathrm{Cl}$ [National Institute on Deafness and Other Communication Disorders]. Most candidates are fitted with unilateral $\mathrm{Cl}$ while some of them, especially children, are implanted in both ears. Previous reports show some binaural advantages in bilateral cochlear implant devices in both children [Lammers et al., 2014] and adults [Olze et al., 2012; Kokkinakis and Pak, 2013; van Schoonhoven et al., 2013]. The use of Cl in subjects with Single Sided Deafness (SSD) was first reported in 2008 [Van de Heyning et al., 2008]. Although their study was primarily designed to investigate the effects of $\mathrm{Cl}$ on tinnitus in an SSD population, they concluded that $\mathrm{Cl}$ is beneficial in specific listening conditions and reported a subjective improvement in daily life [Vermeire and Van de Heyning, 2009].

The yearly incidence of SSD is estimated around one in 30,000 worldwide and often gives rise to difficulties with sound localization and recognition of speech in noise as these functions strongly rely on binaural hearing [Cochlear Clinical background; Nawaz et al., 2014]. In a listening condition where the head is between the sound source and the good ear, the head acts as a barrier reducing the sound intensity reaching the good ear ('the head-shadow effect'). However, the head shadow effect is a physical effect and is not a result of true binaural processing. It is more pronounced at high frequencies, with a magnitude of about $10 \mathrm{~dB}$ at $1000 \mathrm{~Hz}$ up to $20 \mathrm{~dB}$ at $7000 \mathrm{~Hz}$ and typically 8.9-10.7 dB for speech [Shaw, 1974; Schleich et al., 2004]. The squelch effect is the advantage in speech understanding using brain's signal processing of the input from two ears. When speech and noise arrive from different locations, the brain is able to separate them using their distinct interaural timing, loudness, and spectral cues [Vermeire and Van de Heyning, 2009]. Spectral cues are mainly caused by the pinna and important for localization and separation in the vertical plane. The squelch effect results in an improved speech reception threshold (SRT), that is, the signal-to-noise ratio (SNR) at a speech perception score of 50\% [Plomp and Mimpen, 1979], of approximately 3-5 dB in normal hearing subjects [Carhart, 1965]. Furthermore, the advantage of hearing identical signals arriving at both ears compared to one ear is called the binaural summation effect. Here, stimuli from both ears are consolidated which results in a subtle improvement of 1.1-1.9 dB at SRT in normal hearing [Schleich et al., 2004]. The SSD population could in theory benefit from the head shadow effect, squelch effect and binaural summation effect by restoring auditory input in the deaf cochlea. Restoring auditory input in the deaf cochlea can be accomplished by $\mathrm{Cl}$, but is impossible using treatment options like bone conduction systems and contralateral routing of signal systems. Fitted with these alternatives, patients can only be expected to benefit from the head shadow effect [Arndt et al., 2010]. However, the effect of Cl on functional speech reception performance in SSD is not advantageous, by definition. The time delay due to speech processing in $\mathrm{Cl}$ and a suboptimal integration of electric and acoustic stimulation are examples of factors that could limit the binaural speech reception performance in this population.

Some of the previous studies on the effects of $\mathrm{Cl}$ in SSD explicitly reported on auditory training for $\mathrm{Cl}$ rehabilitation [Távora-Vieira et al., 2013; Nawaz et al., 2014; Távora-Vieira et al., 2015]. The SSD population is different from the conventional bilateral severe-to-profound sensorineural hearing loss population as they have one acoustic hearing (AH) ear. This AH ear enables simultaneous stimulation in daily situations and can be expected to promote binaural interaction. Therefore, the question arises what the profit is of intensive formal auditory training and which training program would be optimal for the SSD population. 
Furthermore, standard cochlear implantation has become a viable treatment option for tinnitus complaints in patients with SSD [Van de Heyning et al., 2008; Kleinjung et al., 2009; Arndt et al., 2010; Buechner et al., 2010; Palau et al., 2010; Jacob et al., 2011; Kleine Punte et al., 2011; De Cassia Cassou Guimarães Mendes et al., 2012; Firszt et al., 2012b; Ramos et al., 2012; Távora-Vieira et al., 2013; Hansen et al., 2013; Gartrell et al., 2014; Arts et al., 2015b; Távora-Vieira et al., 2015 and reviewed by Arts et al., 2012; Blasco and Redleaf, 2014; Tokita et al., 2014; Vlastarakos et al., 2014; Van Zon et al., 2015] and intracochlear electrical stimulation that does not encode environmental sounds has been shown to suppress tinnitus both short-term [Rubinstein et al., 2003; Zeng et al., 2011; Chang and Zeng, 2012; Arts et al. 2015a] and long-term [Vernon and Meikle, 2003; Arts et al., in press; unpublished data]. To assess the viability of intracochlear electrical stimulation that does not encode environmental sounds as a potential treatment option for tinnitus, the effects of this meaningless electrical stimulation on speech perception needs to be evaluated.

The aim of the current study was to answer three research questions: 1 . What is the effect of the standard clinical $\mathrm{Cl}$ on functional speech reception performance in SSD-subjects? 2. Is formal auditory training during $\mathrm{Cl}$-rehabilitation in SSD beneficial? and 3 . What is the effect of intracochlear electrical stimulation that does not encode environmental sounds, but suppresses tinnitus, on speech reception performance in SSD?

\section{Materials and Methods:}

The research trial was primarily designed to investigate the effects of intracochlear electrical stimulation that does not encode environmental sounds on tinnitus [Arts et al., 2015b; unpublished data]. The current paper reports on the effects of both standard clinical $\mathrm{Cl}$ and tinnitus specific stimulation (looped electrical stimulation) on speech perception. For a detailed description of the materials and methods used, see the previously published reports. A concise description follows below

\section{Subjects}

Ten SSD subjects were included with an audiometric hearing loss of at least $70 \mathrm{~dB}$ Pure Tone Average (PTA) across $0.5,1$ and $2 \mathrm{kHz}$ in one ear. Their contralateral ear had a moderate to normal audiometric hearing threshold (PTA better than $50 \mathrm{~dB}$ hearing level, $\mathrm{HL}$ ); in case of moderate hearing, a hearing aid (HA) should at least have been tried. The subjects' primary motivation for participating in this study was to reduce their unilateral severe tinnitus, ipsilateral to the deaf ear. Exclusion criteria were medical contraindications for cochlear implantation, diagnosed objective tinnitus, psychiatric disorders and depression.

The average age at time of surgery was 48 years (range: 31-67 years) with an average duration of deafnes of 11 years (range: 2-27 years) with varying etiology (Table 1).

This study was approved by the Ethics Committee of University Maastricht/academic hospital Maastricht and was in accordance with the declaration in Helsinki (approval No. NL38789.068.11). All subjects gave written informed consent prior to participation.
Table 1: demographics

\begin{tabular}{|c|c|c|c|c|c|c|c|c|}
\hline \multirow[t]{3}{*}{ Subject } & \multirow{3}{*}{$\begin{array}{c}\text { Age at time } \\
\text { of surgery } \\
\text { (y) }\end{array}$} & \multirow{3}{*}{ Gender } & \multirow[t]{3}{*}{$\begin{array}{l}\text { Implant } \\
\text { side }\end{array}$} & $\begin{array}{l}\text { Unaided hearing } \\
\text { threshold* }\end{array}$ & \multirow{3}{*}{$\begin{array}{c}\text { Duration of } \\
\text { deafness at time } \\
\text { of surgery (y) }\end{array}$} & \multirow[t]{3}{*}{ Etiology } & \multirow[t]{3}{*}{ Bimodal } & \multirow{3}{*}{$\begin{array}{l}\text { Formal } \\
\text { auditor } \\
\text { training }\end{array}$} \\
\hline & & & & {$[0.5,1,2,4 \mathrm{kHz}](\mathrm{dB})$} & & & & \\
\hline & & & & Implant side & & & & \\
\hline 1 & 59 & Female & Left & $85,95,100,115$ & 8 & $\begin{array}{l}\text { Unknown } \\
\text { Sudden }\end{array}$ & No & No \\
\hline 2 & 38 & Female & Right & $75,75,70,65$ & 7 & $\begin{array}{c}\text { HELLP- } \\
\text { syndrome }\end{array}$ & No & No \\
\hline 3 & 67 & Male & Left & $\mathrm{NR}, \mathrm{NR}, \mathrm{NR}$, NR & 10 & $\begin{array}{l}\text { Morbus } \\
\text { Menière }\end{array}$ & Yes & Yes \\
\hline $\begin{array}{c}4 \text { [Arts et al., } \\
\text { in press] }\end{array}$ & 53 & Male & Right & $N R, N R, N R$, NR & 2 & $\begin{array}{l}\text { Unknown } \\
\text { Sudden }\end{array}$ & No & Yes \\
\hline 5 & 62 & Male & Right & $110,100,110,95$ & 27 & $\begin{array}{c}\text { Viral } \\
\text { Infection }\end{array}$ & Yes & Yes \\
\hline 6 & 50 & Female & Left & $100,105,100,85$ & 10 & $\begin{array}{l}\text { Unknown } \\
\text { progressive }\end{array}$ & No & No \\
\hline 7 & 38 & Female & Left & $\mathrm{NR}, \mathrm{NR}, 105,85$ & 26 & $\begin{array}{l}\text { Morbus } \\
\text { Menière }\end{array}$ & No & Yes \\
\hline 8 & 43 & Female & Left & $\mathrm{NR}, \mathrm{NR}, \mathrm{NR}$, NR & 14 & Infection & No & Yes \\
\hline 9 & 31 & Male & Left & $N R, 110,100,100$ & 2 & Infection & No & No \\
\hline 10 & 35 & Male & Left & $\mathrm{NR}, \mathrm{NR}, \mathrm{NR}, 110$ & 6 & $\begin{array}{l}\text { Unknown } \\
\text { Sudden }\end{array}$ & No & No \\
\hline
\end{tabular}

*: at the third week of Cl-activation; NR: No Response

\section{Design}

Figure 1 shows a schematic presentation of the study design. Subjects who met the inclusion criteria received a $\mathrm{Cl}$ using the mastoidectomy with posterior tympanotomy approach. The electrode array was inserted into the cochlea through the (extended) round window. Four to six weeks post-surgery the standard clinical $\mathrm{Cl}$ fitting was started. This "standard Cl rehabilitation" took about two months. After these two months the $\mathrm{Cl}$ was deactivated for one week followed by the fine tuning procedure to find the optimal stimulation characteristics for tinnitus suppression. 


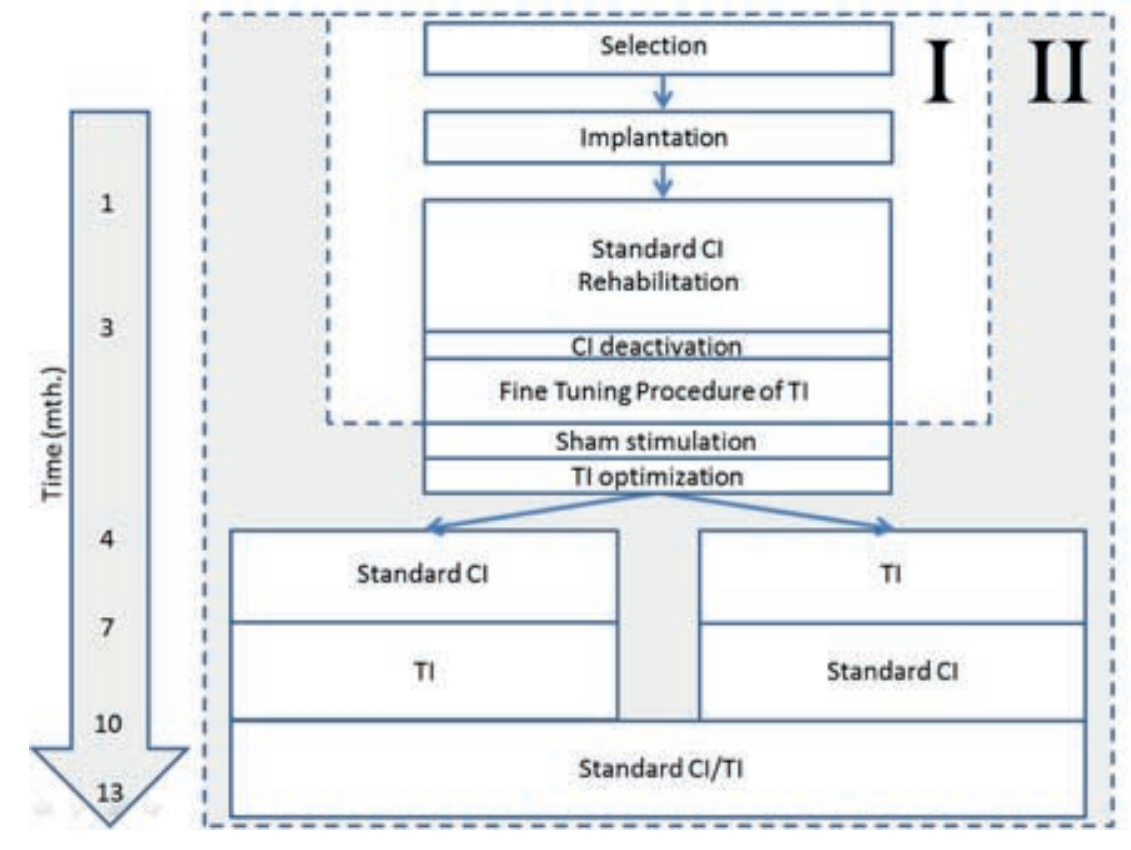

Figure 1: Flowchart. Cl: cochlear implant. This report is about the effects of electrical stimulation on speech perception while previous reports are electrical stimulation on tinnitus [Arts et al., 2015b; unpublished data].

Next, one week of sham stimulation was applied in order to control for possible placebo effects on tinnitus suppression before the preferred stimulation pattern was chosen, based on their experiences in daily use. Subsequently, a crossover design was used in order to compare the effects of both standard clinical $\mathrm{Cl}$ and electrical stimulation that does not encode environmental sounds on tinnitus. Here, either the processor was first programmed for three months as a standard clinical $\mathrm{Cl}$ after which the processor was switched for three months to function as a pattern generator or vice versa. Subjects were randomly assigned to the different treatment arms. Finally, subjects were allowed to choose, based on their experiences, to use their processor either as a speech processor or as a pattern generator for another three-months period. The three-month period of the modality of patient's choice was not considered in this report in order to prevent selection bias.

\section{Device and software}

Subjects were implanted with a MED-EL cochlear implant system (MED-EL Corporation, Innsbruck, Austria), consisting of a CONCERTO implant and the OPUS2 processor. The Flex28 electrode array was used for all but one subject; subject 2 received the Flex24 electrode array for optimal preservation of residual hearing. The OPUS2 was fitted as a speech processor (standard $\mathrm{Cl}$ ) with standard clinical software, Maestro version 4.1.2 and 6.0 using MED-EL's Diagnostic Interface Box II or MAX programming interface while the OPUS2 was programmed as a pattern generator for tinnitus suppression using customized software (Inst. of Mechatronics, Innsbruck, Austria) in Matlab version 7.11.0 (R2010b) ) (The Mathworks, Natick, MA, USA) using MED-EL's Diagnostic Interface Box II. The used subject-specific electrical stimulation of the pattern generator for tinnitus suppression is shown in a previous report [unpublished data].

\section{Auditory training}

A random five out of the ten included subjects received formal auditory training (Table 1). This forma auditory training, based on the Dutch training program for conventional Cl-candidates, was provided in the clinic by a speech therapist with considerable experience in Cl-rehabilitation. The training started one week after $\mathrm{Cl}$-activation on a weekly basis for the first three weeks followed by a session in week 7 and 8 accompanied with practicing at home. The intensity of the formal auditory training was similar to the auditory training during $\mathrm{Cl}$-rehabilitation in patients with bilateral severe-to-profound hearing loss. Training first focused on simple detection of sounds, progressing with time towards more complex auditory abilities like recognition and distinction of simple sounds, recognition of vowels and recognition of words and sentences. In order to optimize training using sound originating from the $\mathrm{Cl}$-ear, the contralateral ear was shielded at home using an earplug (E-A-Rsoft ${ }^{\mathrm{TM}} \mathrm{FX}$ ) and in the clinic using an earplug together with an earmuff (E-A-R Ultra 9000). If applicable, the contralateral HA was deactivated during training.

Independent of whether they received formal auditory training, all ten subjects were provided with Dutch audiobooks including the written versions, mp3-files of Dutch monosyllabic words and an audio cable (Adapter Cable, EXT mode) that connected the processor to the audio source. They were informed to train their hearing by listening to and read the books concomitantly. At a later stage we suggested to use the audiobooks without visual help. No requirements were imposed on the intensity of this training. The subjects who received formal auditory training were advised to start with the audiobooks after the formal auditory training was finished.

\section{Outcome measurements}

\section{Speech reception in quiet}

Speech discrimination scores in quiet were tested at 55,65 and $75 \mathrm{~dB}$ sound pressure level (SPL) using open-set Dutch monosyllable words (Consonant-Nucleus-Consonant, CNC) [Bosman et al., 1995] in free-field-condition in a sound-treated booth. Subjects were seated 1 m away in front (0 degrees azimuth) of the speaker. The contralateral AH ear was shielded using an earplug and earmuff to prevent a ceiling effect in the condition using the standard clinical $\mathrm{Cl}$ (median of preoperative unaided phoneme score at $50 \mathrm{~dB} \mathrm{SPL}: 82.5 \%$ correct). No shielding was performed for the condition using tinnitus specific electrical stimulation. Speech discrimination scores in quiet were collected in the seventh week of the $\mathrm{Cl}$ rehabilitation, and after three months during both standard $\mathrm{Cl}$ use and tinnitus specific stimulation during the crossover design. Speech discrimination scores were additionally obtained using the Cl's direct input to eliminate the AH ear completely. For this purpose, an Adapter Cable (EXT mode) was used to connect an $\mathrm{mp3}$-player to the processor. Here, a comfortable loudness level was used to present similar lists of CNC-words. The speech discrimination scores were obtained in an open-set via direct input at the end of the $\mathrm{Cl}$-rehabilitation.

\section{Speech reception in noise}

The speech perception in noise test used in the current study was performed earlier in SSD-subjects with a Cl by Vermeire and Van de Heyning [2009]. Speech perception in noise was tested using the Leuven Intelligibility Sentence Test (LIST) [Van Wieringen and Wouters, 2008] consisting of 35 lists of 10 sentences each, spoken by a female speaker in a speech-weighted stationary noise. An adaptive one-down, one-up procedure with a constant noise level of $65 \mathrm{~dB}$ SPL was used to avoid possible floor and ceiling effects. The level of speech signal was adaptively varied to estimate the SRT [Plomp and Mimpen, 1979]. The initia speech level was $55 \mathrm{~dB} \mathrm{SPL}$. After each sentence the signal level was adjusted in steps of $2 \mathrm{~dB}$ according to subject's response. Two consecutive lists were presented per condition and the SRT was calculated by 
averaging the SNRs of the second list that was presented [Plomp and Mimpen. 1979; Vermeire and Van de Heyning, 2009]. The test was performed in a free-field-condition in a sound-treated booth with the speakers positioned $1 \mathrm{~m}$ away from the subject, separated by $90^{\circ}$ azimuth. Three spatial configurations were included; both speech and noise presented from the front (SONO), speech presented from the front and noise from the implant-side (SONi), and noise presented from the front and speech presented from the implant-side (SiNO). Moreover, monaural (listening with the AH ear only: bimodal hearing subjects used their conventional $\mathrm{HA}$ ) and binaural listening conditions (listening with both the $\mathrm{AH}$ ear and the implanted ear) were included. The speech perception in noise test was performed at the end of the $\mathrm{Cl}$ rehabilitation, and after three months of both standard $\mathrm{Cl}$ use and tinnitus specific stimulation during the crossover design. The order of spatial configurations and listening conditions was randomized across subjects and measurements.

\section{Speech, Spatial and Quality of hearing questionnaire}

To evaluate the subjective improvement of $\mathrm{Cl}$ the Speech, Spatial and Qualities of hearing scale (SSO) was used [Gatehouse and Noble, 2004]. This questionnaire consists of three sections; hearing speech, spatia hearing and qualities of sound, with a total of 49 items. Each item was scored by placing a mark on a ruler, marked from 0 (minimal ability) to 10 (complete ability). The questionnaire was filled out prior to the surgery, at the end of the $\mathrm{Cl}$-rehabilitation, and after three months of both standard $\mathrm{Cl}$ use and tinnitus specific stimulation during the crossover design. When answering the questions, the subjects were instructed to consider their experiences in everyday listening condition.

\section{Discomfort due to the Cl}

In order to investigate the effect of formal auditory training during $\mathrm{Cl}$-rehabilitation in SSD, changes in comfort related to $\mathrm{Cl}$-use during $\mathrm{Cl}$-rehabilitation were assessed using a Visual Analogue Scale (VAS) from 0 to 10 . At the left end of the scale ' 0 No Discomfort' and at the right ' 10 Maximum Discomfort' was indicated. Values were accurate to one decimal place. The discomfort due to the $\mathrm{Cl}$ was scored during the fitting sessions in the standard Cl-rehabilitation; in the second, third, fourth, seventh and eighth week.

\section{Statistic}

Statistics were performed with IBM SPSS Statistics, version 22, and $p$ values smaller than 0.05 were, unless otherwise reported, considered statistically significant.

\section{Standard Cl rehabilitation}

Speech discrimination scores in quiet, speech reception in noise and results of the SSQ were analyzed using one-sided Wilcoxon signed-rank tests (Exact). A one-sided one-sample Wilcoxon signed-rank tests (Exact) was used for speech perception via direct input (median equals zero). In order to assess the effect of formal auditory training, one-sided Mann-Whitney $U$ tests (Exact) were performed. Bonferroni corrections for multiple comparisons were applied. Furthermore, in order to assess the effect of formal auditory training on $\mathrm{Cl}$ related discomfort during $\mathrm{Cl}$-rehabilitation, a two-sided repeated measures analysis of variance ( $\mathrm{rANOVA}$ ) was performed.

\section{Crossover design}

To study the effects of intracochlear electrical stimulation that does not encode environmental sounds on hearing, results obtained during the crossover design were used. Two-sided Mann-Whitney U tests (Exact) were performed to determine possible carryover effects and period effects while one-sided Mann-Whitney $\mathrm{U}$ tests (Exact) were performed to determine possible treatment effects. A carryover effect in a crossover design is when the effects of one or both interventions during the first active period have a residual biological effect during the second active period. Testing for possible carryover effects is important as using this methodological design no washout period was used. For the carryover effects, $p$ values smaller than 0.10 were considered statistically significant as the power of the test to detect carryover effects is not great [Grizzle, 1965]. In case of a detected carryover effect, the second-period data are not used because they provide a biased estimate of treatment effects. Period effects show a clear preference for the former or latter period and could bias treatment effects as well. Treatment effects were used to determine the effectiveness of the standard clinical $\mathrm{Cl}$ compared to stimulation that does not encode environmental sounds. Finally, descriptive statistics and two-sided one-sample Wilcoxon signed-rank tests (Exact) were applied to describe the effects of stimulation that does not encode environmental sounds on hearing, compared to no implant use (median equals zero).

\section{Results:}

Figure 2 shows the contralateral hearing thresholds at time of the selection procedure. Unaided audiometric hearing thresholds were obtained for all but two subjects; for subject 3 and 5 the audiometric hearing thresholds were obtained in the aided condition (left). The summarized unaided hearing thresholds are shown for both the "no contralateral HA" group (upper right) and the "bimodal" group (lower right). Subjects 2 had an extended Cl-rehabilitation period as she experienced problems during the fitting procedure with optimizing and stabilizing the dynamic range.
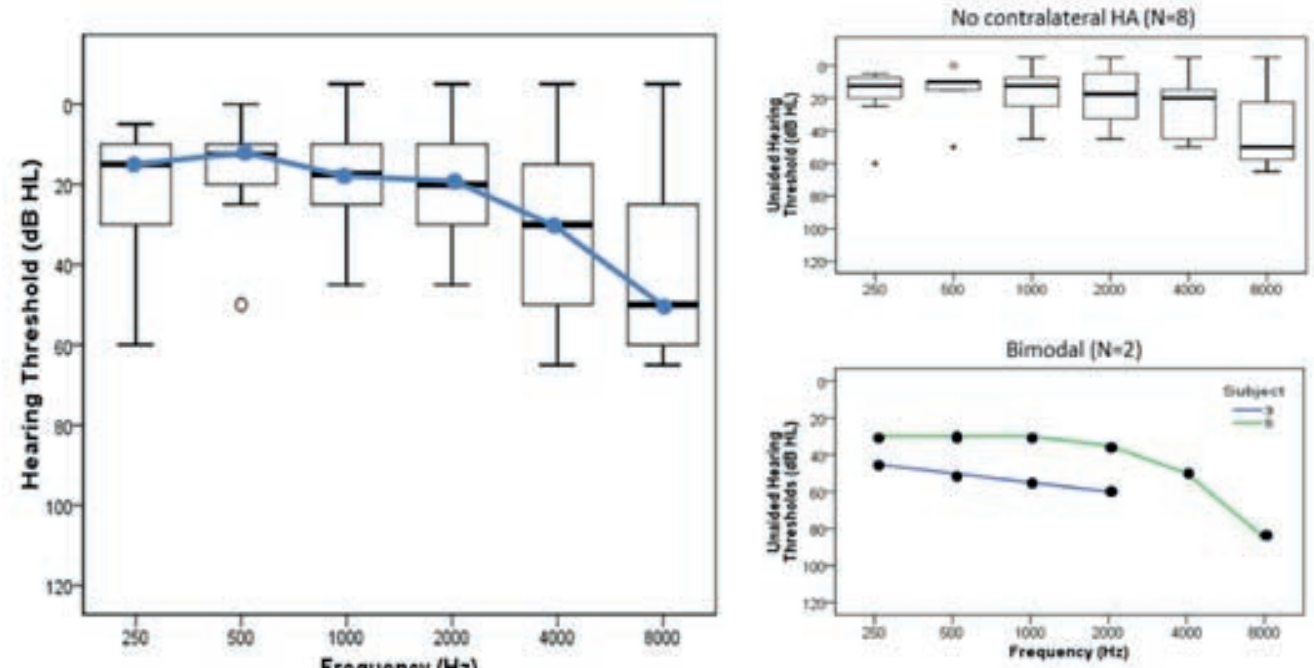

Figure 2: Summarized contralateral hearing thresholds. Unaided audiometric hearing thresholds were obtained for all but two subjects: for subject 3 and 5 the hearing thresholds were obtained in the aided condition (left). The summarized

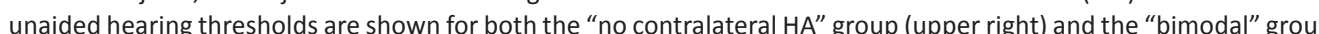
(lower right). The figure shows minimum, first quartile, median, third quartile, maximum, and outliers. 


\section{Speech reception using standard $C$}

In order to assess the effect of the standard clinical $\mathrm{Cl}$ on speech reception, the benefit of adding the $\mathrm{Cl}$ on speech discrimination scores in quiet was measured both in free-field conditions with a shielded $\mathrm{AH}$ ear to prevent ceiling effects (figure 3 , left graph) and via direct audio input (figure 3, right graph). Even with the $\mathrm{Cl}$-processor turned off, larger phoneme scores are observed at higher stimulus intensities, which is possibly related to limited shielding of the contralateral $\mathrm{AH}$ ear. However, at each intensity, a statistically significant profit of adding the $\mathrm{Cl}$ was found $(p=0.001, p=0.001$ and $p=0.002$ at 55,65 and $75 \mathrm{~dB} \mathrm{SPL}$ respectively). Furthermore, a statistically significant advantage of the $\mathrm{Cl}$ was found via direct input $(p=0.002)$.
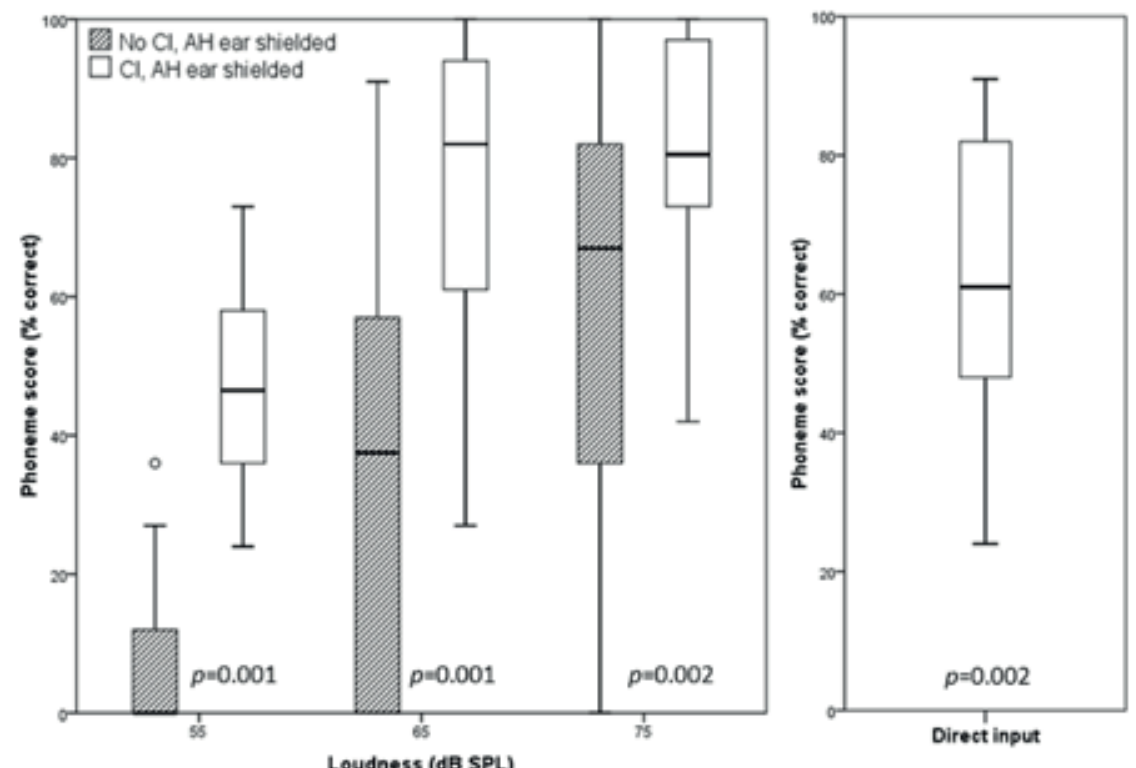

Figure 3: Speech discrimination scores in quiet using CNC words in a free-field-condition (left graph) and via direct input (right graph). In the free-field-condition the $\mathrm{AH}$ ear was shielded. The figures show minimum, first quartile, median, third quartile, maximum, and outliers. A statistically significant benefit of adding the $\mathrm{Cl}$ was found at 55 , 65 and $75 \mathrm{~dB}$ SPL. Furthermore, a statistically significant benefit of the $\mathrm{Cl}$ was found via direct input.

Results of the speech in noise measurements are shown in Figure 4: SRTs for both monaural (AH only) and binaural ( $\mathrm{AH}$ and $\mathrm{Cl}$ ) listening conditions are shown. A statistically significant advantage of adding the $\mathrm{Cl}$ was found only in the SiNO configuration $(p=0.009)$; in the other two spatial configurations, no significant differences of adding the $\mathrm{Cl}$ were observed. Comparing the monaural conditions of the SONO and SONi spatial configurations shows a positive head shadow effect. After adding the $\mathrm{Cl}$, this effect appears to be still present and equally strong. Interestingly, the negative head shadow effect by comparing the monaural conditions of the SONO and SiNO configurations was partially offset by adding the $\mathrm{Cl}$. In order to evaluate the spatial release from masking, the results obtained in the binaural listening condition when speech and noise were co-located (SONO) were compared to the results obtained in the binaural listening condition when speech and noise were spatially separated (SONi) [Gartrell et al., 2014]. Here, a statistically significant benefit of separating speech and noise was found $(p=0.006)$.

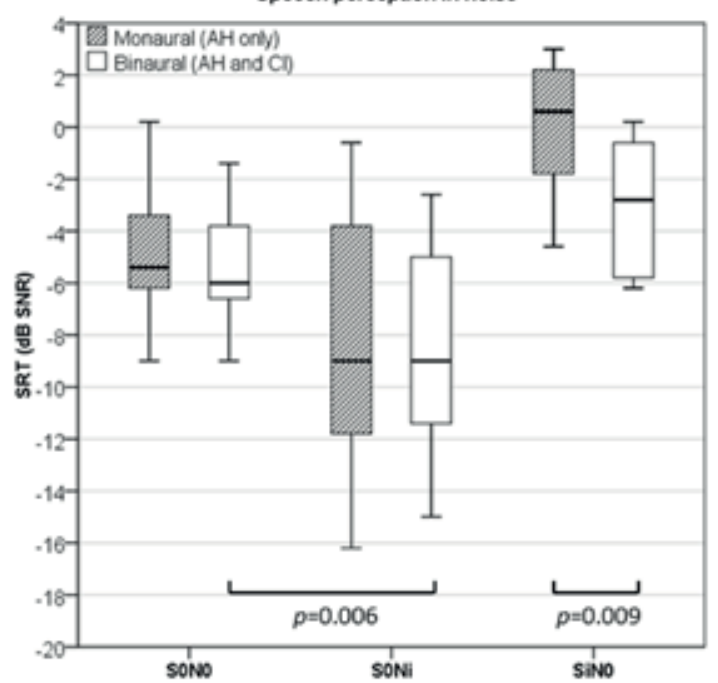

Figure 4: Speech reception in noise after approximately two months of standard Cl-use. The figure shows minimum, first quartile, median, third quartile and maximum. A statistically significant benefit of adding the $\mathrm{Cl}$ was found in the SiNO configuration. Moreover, the SRT in the binaural listening condition when speech and noise were spatially separated (SONi) was statistically improved compared to the binaural listening condition when speech and noise were co-located (SONO).

Figure 5 shows the subjective ability of hearing using the SSQ scored prior to the surgery and after approximately two months of $\mathrm{Cl}$-use. Although not statistically significant for all, slight improvements were seen in all subscales by adding the $\mathrm{Cl}$. However, a statistically significant improvement in the experienced spatial hearing ability was observed $(p=0.007)$.

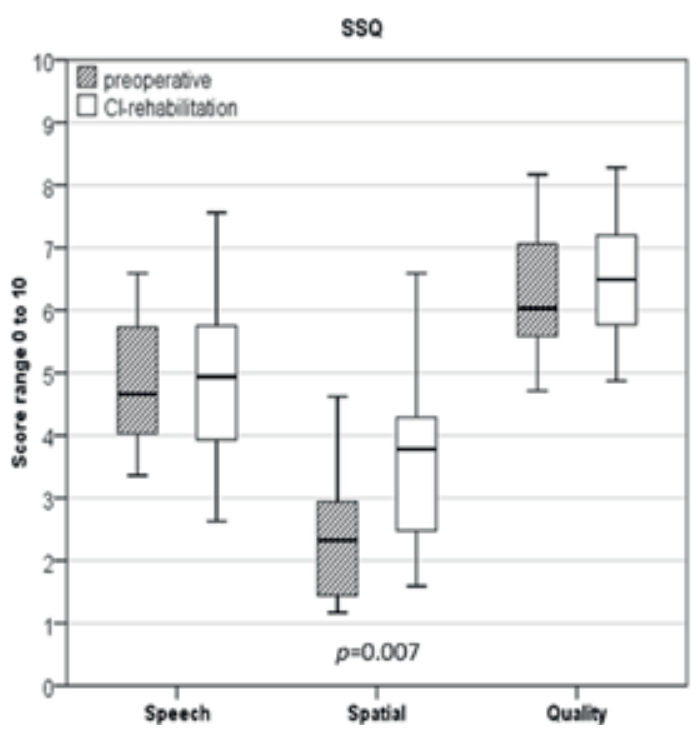

Figure 5: The subjective ability of hearing using the SSQ. The figure shows minimum, first quartile, median, third quartile and maximum. A statistically significant improvement in the subjective ability of hearing was found for spatial hearing after approximately two months of standard Cl-use compared to the preoperative ability of hearing. 
Some subscales of the speech-related and quality-related items of the SSQ are shown in Figure 6. Here, the "speech in noise" (average of item 1, 4, 5 and 6 of the speech scale), "speech in speech contexts" (average of item 7, 8, 9 and 11 of the speech scale), "multiple speech streams processing and switching" (average of item 10,12 and 14 of the speech scale), "segregation of sounds" (average of item 1, 2 and 3 of the quality scale) and "listening effort" (average of item 14,15 and 18 of the quality scale) subscales wer presented as in these subscales the largest effect of adding the $\mathrm{Cl}$ were initially expected (Gatehouse and Akeroyd, 2006). A statistically significant improvement in the subjective ability of hearing was found for the listening effort subscale after two months of $\mathrm{Cl}$-use compared to the preoperative ability of hearin $(p=0.005)$.

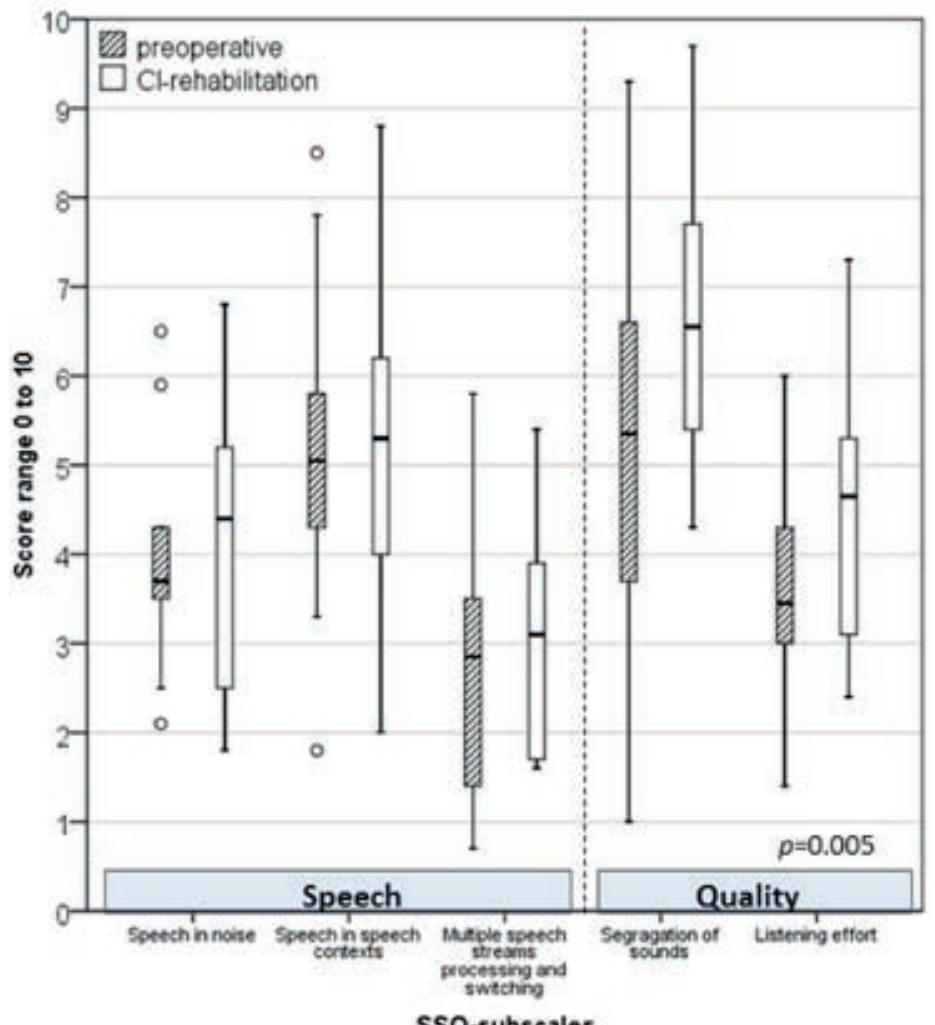

SSQ-subscales Figure 6: The effect of $\mathrm{Cl}$ on the subjective ability of hearing using different SSQ-subscales. The figures show the subjective ability of hearing was found for the listenin effort subsca ste after aproximately two months standard Cl-use compared to the preoperative ability of hearing.

\section{Formal auditory training}

Figure 7 shows the effect of formal auditory training on speech perception in quiet via direct input (left graph), speech perception in noise (middle graph) and SSQ (right graph). Absolute scores of speech discrimination in quiet are shown, while the benefit is shown for speech discrimination in noise (monaural vs. binaural) and SSQ (after two months of Cl-use vs. preoperatively obtained results). No statistically significant benefit of formal auditory training was found for speech perception in quiet via direct input, the three spatial configurations in the speech perception in noise test and for the three subscales of the SSO
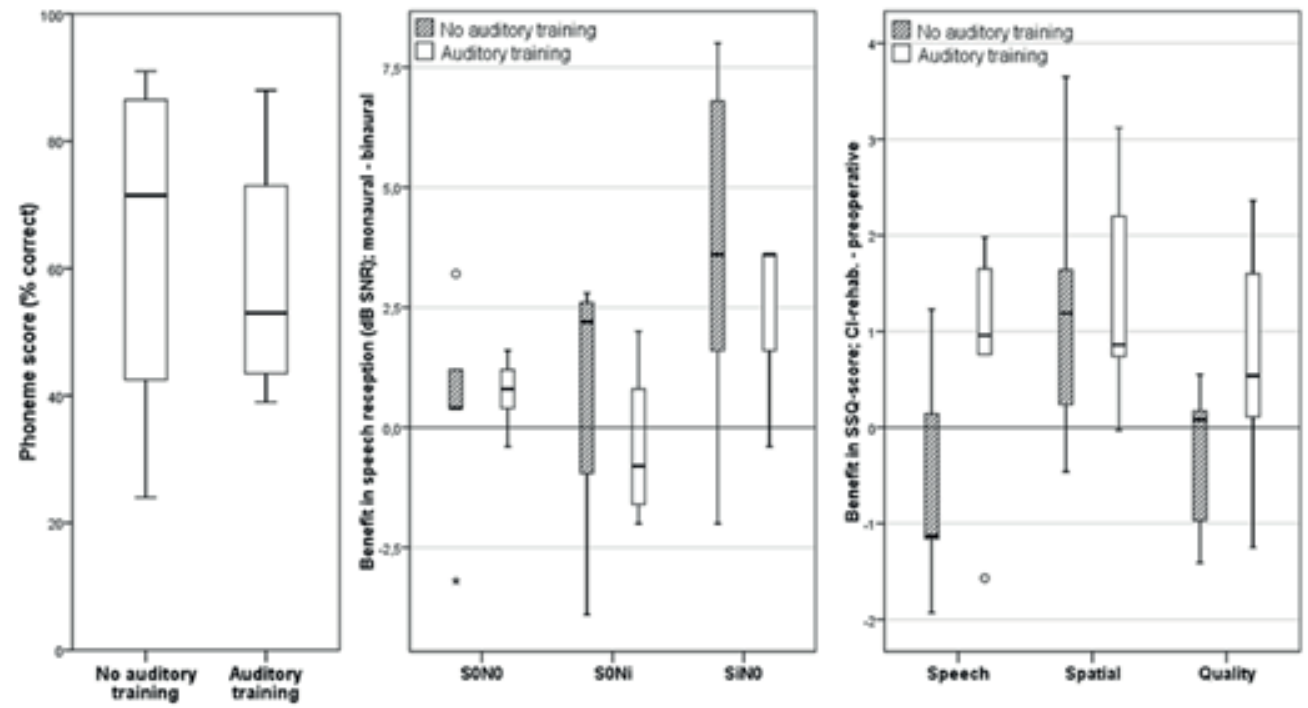

Figure 7: The effect of formal auditory training on direct-audio speech reception in quiet (left graph), speech reception in noise (middle graph) and SSQ-subscales (right graph). The figures show minimum, first quartile, median, third quartile, maximum, and outliers.

Figure 8 shows the effect of formal auditory training on $\mathrm{Cl}$-related discomfort during standard $\mathrm{C}$ rehabilitation, as measured with a VAS. A statistically significant benefit of formal auditory training on $\mathrm{Cl}$ related discomfort was observed $(p=0.049)$. Cl-related discomfort decreased substantially faster in subjects with, compared to subjects without formal auditory training. 


\section{Cl related discomfort}

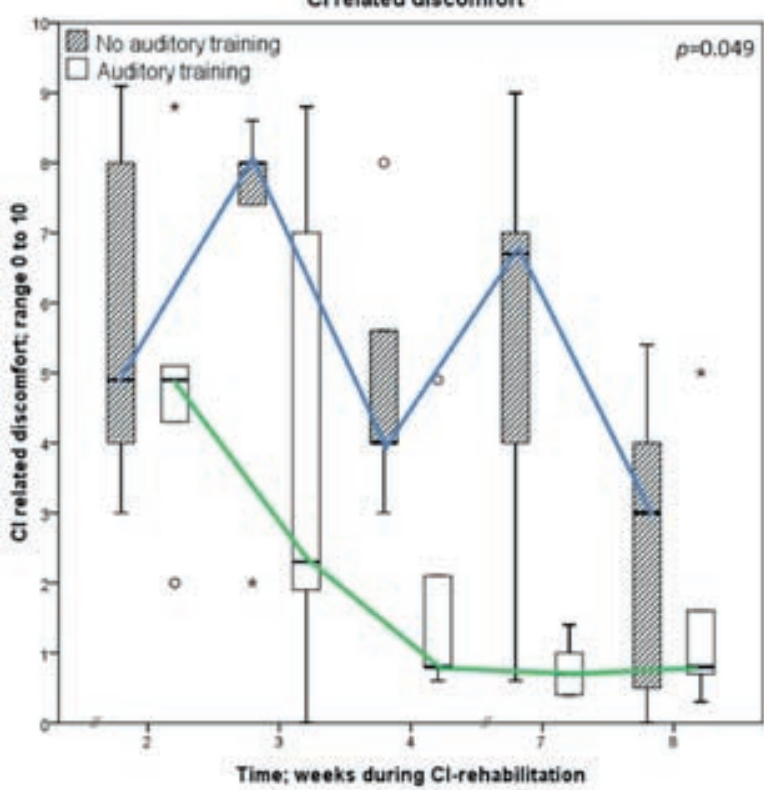

Figure 8: The effect of formal auditory training on Cl related discomfort in the second, third, fourth, seventh and eighth week during standard Cl-rehabilitation. The discomfort was scored using a VAS. The figure shows minimum first quartile, median, third quartile, maximum, and outliers. A statistically significant benefit of formal auditory training on Cl related discomfort was obtained during the standard CI-rehabilitation.

\section{Speech reception using the pattern generator}

The effects of intracochlear electrical stimulation that does not encode environmental sounds (pattern generator) and standard clinical $\mathrm{Cl}$ on speech reception are shown in table 2. Here, a crossover design is used for the two different $\mathrm{Cl}$ modes (pattern generator or standard clinical $\mathrm{Cl}$ ). Each $\mathrm{Cl}$ mode was used for three months.

As shown in table 2, carryover effects were found in the SONO and SONi spatial configurations of the speech perception in noise test. Therefore, to estimate the treatment effect the data was additionally analyzed (for these spatial configurations) after excluding the second period data in the crossover design (Fig.1). No period effects were found in any of the outcomes measures. When comparing the effects of the pattern generator with the effects of the clinical $\mathrm{Cl}$ ('treatment effect'), a statistically significant advantage of the standard clinical $\mathrm{Cl}$ compared to the pattern generator was found for all tested intensity levels in speech perception in quiet ( $55 \mathrm{~dB} \mathrm{SPL}: p=0.032,65 \mathrm{~dB} \mathrm{SPL}: p=0.008$ and $75 \mathrm{~dB}$ SPL: $p=0.032$ ). The profit of binaural hearing ( $\mathrm{AH}$ ear and pattern generator/standard clinical $\mathrm{Cl}$ ) compared to monaura hearing (AH ear only) in speech perception in noise is shown for the three tested spatial configurations. A statistically significant benefit of the standard clinical $\mathrm{Cl}$ compared to the pattern generator was obtained for the SONi and SiNO spatial configuration ( $p=0.020$ and $p=0.016$ respectively). Furthermore, the subjective advantage of adding the implant in the ability of hearing is shown for the three SSQ-subscales. Here, results obtained during the crossover design were compared to the preoperative ability of hearing. A statistically significant benefit of the standard clinical $\mathrm{Cl}$ compared to the pattern generator was observed for the SSQ-speech subscale ( $p=0.048)$; other subscales showed no statistically significant profit of $\mathrm{Cl}$ compared to pattern generator.
Table 2: Crossover design.

\begin{tabular}{|c|c|c|c|c|c|c|c|}
\hline & \multicolumn{2}{|c|}{ Implant function } & \multirow{2}{*}{ 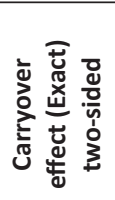 } & \multirow{2}{*}{ 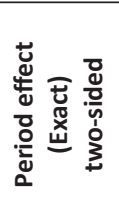 } & \multirow{2}{*}{ 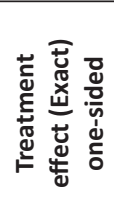 } \\
\hline & & & $\begin{array}{r}\text { Pattern generator } \\
\mathrm{N}=10\end{array}$ & $\begin{array}{r}\text { Standard clinical Cl } \\
\qquad \mathrm{N}=10\end{array}$ & & & \\
\hline \multicolumn{8}{|c|}{3 months treatment } \\
\hline \multirow{5}{*}{$\begin{array}{c}\text { Speech } \\
\text { discrimination in } \\
\text { quiet }^{2}\end{array}$} & \multirow[t]{3}{*}{$55 \mathrm{~dB} \mathrm{SPL}$} & Mean & 3.60 & 25.80 & \multirow[t]{3}{*}{$p=0.135$} & \multirow[t]{3}{*}{$p=0.421$} & \multirow[t]{3}{*}{$p=0.032$} \\
\hline & & SD & 7.183 & 31.850 & & & \\
\hline & & Median (IQR) & $1.50(-0.75-7.50)$ & $22.50(4.50-60.75)$ & & & \\
\hline & \multirow{3}{*}{$65 \mathrm{~dB}$ SPL } & Mean & 0.60 & 30.30 & \multirow[t]{3}{*}{$p>0.999$} & \multirow[t]{3}{*}{$p=0.968$} & \multirow[t]{3}{*}{$p=0.008$} \\
\hline & & SD & 4.195 & 28.087 & & & \\
\hline \multirow{4}{*}{$\begin{array}{l}\text { (percentage } \\
\text { points) }\end{array}$} & & Median (IQR) & $0.00(-1.50-3.75)$ & $30.00(9.75-48.00)$ & & & \\
\hline & \multirow[t]{3}{*}{$75 \mathrm{~dB} \mathrm{SPL}$} & Mean & 0.30 & 19.50 & \multirow[t]{3}{*}{$p=0.802$} & \multirow[t]{3}{*}{$p=0.952$} & \multirow{3}{*}{$p=0.032$} \\
\hline & & SD & 3.302 & 26.805 & & & \\
\hline & & Median (IQR) & $0.00(-0.75-3.00)$ & $13.50(-3.00-45.00)$ & & & \\
\hline \multirow{6}{*}{$\begin{array}{c}\text { Speech } \\
\text { perception in } \\
\text { noise }^{b}\end{array}$} & \multirow[t]{3}{*}{ SONO } & Mean & 0.56 & 0.08 & \multirow[t]{3}{*}{$p=0.032^{\circ}$} & \multirow[t]{3}{*}{$p=0.984$} & \multirow{3}{*}{$\begin{array}{c}p=0.659 \\
(p=0.099)\end{array}$} \\
\hline & & SD & 1.296 & 1.482 & & & \\
\hline & & Median (IQR) & $0.80(-0.50-1.60)$ & $0.40(-0.90-1.20)$ & & & \\
\hline & \multirow[t]{3}{*}{ SONi } & Mean & 0.00 & 1.20 & \multirow[t]{3}{*}{$p=0.048^{\circ}$} & \multirow[t]{3}{*}{$p=0.913$} & \multirow{3}{*}{$\begin{array}{c}p=0.206 \\
(p=0.020)\end{array}$} \\
\hline & & SD & 1.033 & 1.987 & & & \\
\hline & & Median (IQR) & $0.20(-0.60-0.90)$ & $1.80(-0.20-2.80)$ & & & \\
\hline \multirow[t]{3}{*}{ (dB SNR) } & \multirow[t]{3}{*}{ SiNO } & Mean & -0.32 & 2.32 & $p>0.999$ & $p=0.238$ & $p=0.016$ \\
\hline & & SD & 1.777 & 3.045 & & & \\
\hline & & Median (IQR) & $0.20(-1.50-1.00)$ & $2.60(-0.80-4.80)$ & & & \\
\hline & Speech & Mean & -0.86 & 0.13 & $p=0.222$ & $p>0.999$ & $p=0.048$ \\
\hline & & SD & 1.054 & 2.015 & & & \\
\hline & & Median (IQR) & $-0.78(-1.93-0.17)$ & $0.40(-1.88-1.51)$ & & & \\
\hline & Spatial & Mean & 0.91 & 2.11 & $p=0.841$ & $p>0.999$ & $p=0.111$ \\
\hline SSQ $^{c}$ & & SD & 0.918 & 2.240 & & & \\
\hline & & Median (IQR) & $0.67(0.29-2.06)$ & $1.26(0.56-4.74)$ & & & \\
\hline & Quality & Mean & 0.03 & 0.70 & $p=0.310$ & $p>0.999$ & $p=0.155$ \\
\hline & & SD & 0.807 & 1.167 & & & \\
\hline & & Median (IQR) & $0.23(-0.63-0.57)$ & $0.49(-0.13-1.80)$ & & & \\
\hline
\end{tabular}

dB SNR: the signal-to-noise ratio in decibels, CI: cochlear implant, IQR: interquartile range, SD: standard deviation SSQ: Speech, Spatial and Qualities of hearing scale, SONO: both speech and noise presented from the front, SONi speech presented from the front and noise from the implant-side, SiNO: speech resented from the implant-side and noise from the front. ${ }^{a}$ The benefit in correct phoneme score of adding the implant function (by subtracting the percentage correct phoneme score in the monaural condition (AH ear only) from the percentage correct phoneme score in the binaural condition (AH ear and implant function). This benefit is expressed in percentage points. In the standard clinical Cl-condition the AH ear was shielded to prevent a possible ceiling effect. ${ }^{b}$ The benefit in $\mathrm{dB}$ SNR at SRT of adding the implant function (by subtracting the signal-to-noise ratio at SRT in the binaural condition (AH ear and implant function) from the signal-to-noise ratio at SRT in the monaural condition (AH ear only)). c The benefit of adding the implant function (by subtracting the preoperative SSQ-scores from the SSQ-scores obtained during the crossover design). ${ }^{\circ}$ The second-period data was excluded to estimate the treatment effect (in parentheses) due to the identified carryover effect. 
Finally, the effect of the pattern generator per se on speech perception in quiet, speech reception in noise and the subjective ability of hearing was statistically analyzed using a hypothesized median that equals zero (no effect). Results show that the pattern generator has no statistically significant effect (neither advantageous nor disadvantageous on any of the hearing-related outcomes measures in Table 2, except for the speech and spatial subscales of the SSQ ( $p=0.010$ and $p=0.005$ respectively). The pattern generator shows a negative benefit (disadvantageous) on the speech related subjective ability of hearing while a positive benefit (advantageous) was found on the spatially related subjective ability of hearing.

\section{Discussion:}

For the included SSD-participants, the current study shows a statistically significant improvement in speech perception in quiet with a $\mathrm{Cl}$ after approximately two months of $\mathrm{Cl}$-use. Speech reception in noise improved statistically significant with $\mathrm{Cl}$ when speech is presented from the implant-side and noise is presented from the front. Furthermore, a statistically significant improvement of the spatially related subjective ability of hearing and listening effort was obtained with Cl use. No statistically significant profit of formal auditory training was obtained during the $\mathrm{Cl}$ rehabilitation in speech perception in quiet, speech reception in noise and the subjective ability of hearing while a statistically significant benefit was found in $\mathrm{Cl}$-related discomfort.

Compared to electrical stimulation that does not encode environmental sounds, the standard $\mathrm{Cl}$ was significantly better at least to some extent in all tested conditions of speech perception in quiet, in speech perception in noise when speech and noise were spatially separated, and in the subjective ability of speech related hearing using the SSQ. Furthermore, electrical stimulation that does not encode environmental sounds was neither statistically advantageous nor disadvantageous in speech perception in quiet and speech perception in noise. A significant disadvantage was obtained in the SSQ-speech-scale while a statistically significant advantage was obtained in the SSQ-scale related to spatial hearing.

\section{Speech reception using standard $C$}

Shielding the better ear in order to prevent ceiling effects in speech perception in quiet resulted in an unrealistic situation which makes the interpretation of some results complex. However, the speech perception in quiet measured using this methodology was consistent with previous studies [Stelzig et al., 2011; Firszt et al., 2012b]. As far as the authors know, this is the first study in SSD using direct input to obtain speech discrimination scores in quiet of the $\mathrm{Cl}$ only condition, which appears to be a valid method to be used in this specific group of listeners with severe asymmetric hearing. These results are consisten with the speech discrimination scores in quiet clinically obtained with $\mathrm{Cl}$ in the bilateral severe-toprofound sensorineural hearing loss population [Chen et al., 2001].

The obtained speech in noise results, after two months of $\mathrm{Cl}$-use are consistent with previous result obtained after 12 months of $\mathrm{Cl}$-use in a similar group of subjects as indicated in the study by Vermeire and Van de Heyning [2009]. Both their and our studies show a statistically significant improvement of speech reception in noise in binaural hearing ( $\mathrm{AH}$ and $\mathrm{Cl}$ ) compared to monaural hearing ( $\mathrm{AH}$ only). This benefit is a combination of squelch effect and head shadow effect. Head shadow effect could not directly be measured in the current study due to difficulties in masking the AH ear. The observed binaural summation effect (which was evaluated by the addition of the $\mathrm{Cl}$ when both speech and noise were presented from the front) and squelch effect (which was evaluated by the addition of the $\mathrm{Cl}$ when speech was presented from the front and noise was presented from the implant-side) were not statistically significant in both studies. The binaural masking level difference to measure squelch effect is suggested in future studies as it is a more sensitive measurement compared to the used speech in noise test [Snik et al., 2015]. Simila to previous reports [Arndt et al., 2010; Buechner et al., 2010; Távora-Vieira et al., 2012] but different from Vermeire and Van de Heyning [2009], no deterioration was found as a result of $\mathrm{Cl}$ activation in speech reception in noise when speech was presented from the front and noise from the implant-side.

Based on the current results, one could state that tinnitus suppression by the $\mathrm{Cl}$ is a confounder of speech reception in noise in binaural hearing: the observed SRT improves by tinnitus suppression, even contralaterally [Mertens et al., 2013; Gartrell et al., 2014]. In order to control for this potential confounde the spatial release from masking was additionally evaluated. In the binaural listening condition, statistically significant benefit of separating speech and noise was found.

The SSQ scale related to spatial hearing was significantly improved after two months of Cl-use compared to the preoperative rating. The speech and quality subscales also showed a slight improvement, but did not reach the level of significance. These results are partially consistent with Vermeire and Van de Heyning [2009] who found statistically significant improvements on all three subscales after 12 months of Cl-use. Firszt and colleagues [2012a] show a statistical improvement of the speech and spatial subscale after 6 months of $\mathrm{Cl}$-use in 10 adults with asymmetric hearing loss. The results in the current study can probably be explained by the significantly shorter follow-up since previous studies show an improvin speech perception up to at least 12 [Arndt et al., 2011; Hassepass et al., 2012] and 36 months of Cl-use [Mertens et al., 2015].

\section{Formal auditory training}

Our study shows no statistically significant profit of formal auditory training on either of the hearing related outcomes (speech reception in quiet and noise and SSQ). However, a statistically significant benefit was found during $\mathrm{Cl}$ rehabilitation in $\mathrm{Cl}$-related discomfort. To the best of our knowledge, this is the first study investigating the effects of formal auditory training for Cl in SSD patients. From the literature reporting on auditory training after cochlear implantation in SSD, Nawaz and colleagues [Nawaz et al., 2014] used a formal auditory training with the audiologist and at home on a daily basis. They used an mp3-player plugged to a 2 channels attenuation box connected to both the $\mathrm{Cl}$ using a direct input cable and the acoustic ear using an insert phone. Távora-Vieira and colleagues [Távora-Vieira et al., 2013] asked their subjects to listen on a daily basis to audiobooks through the direct audio input of the processor. Subjects were initially recommended to listen and read the book concomitantly. Afterwards they listened to the same part of the book again without visual help. In a more recent study, Távora-Vieira and colleagues [Távora-Vieira et al., 2015] reported on a similar protocol starting with meaningful words a for example numbers, colors and animals prior to the audiobooks. In contrast, the formal auditory raining used in the current study consisted of intensive training with a speech therapist on a weekly basis using a shielded contralateral ear.

\section{Speech reception using the pattern generator}

Electrical stimulation using the pattern generator does not encode environmental sounds and was neither statistically advantageous nor statistically disadvantageous in speech perception in quiet and speech reception in noise. Loudness adaptation to the stimulus provided by the pattern generato [unpublished data] might contribute to this positive result, indicating a good acceptability of this tinnitus suppressive device. A statistically significant disadvantage was however obtained in the speech related SSO scale while a statistically significant advantage was found in the spatial SSO subscale. The subjective disadvantage of the pattern generator on the speech related ability of hearing while no disadvantage was observed in speech perception in quiet and noise may be explained by the improvement of speech 
perception during standard $\mathrm{Cl}$ rehabilitation. Using this standard $\mathrm{Cl}$, subjects seem to develop new internal standards for their subjective ability of hearing.

Finally, the standard $\mathrm{Cl}$ was significantly better than the pattern generator in all tested conditions of speech perception in quiet, for some of the tested spatial configurations of the speech perception in noise test and in the subjective ability of hearing using the SSQ. Noteworthy, suboptimal effects of standard Cl during the used crossover design seem to be plausible due to the intermittent standard Cl follow-up (when using e.g. the pattern generator). Nevertheless, a significant benefit of the standard $\mathrm{Cl}$ on speech perception was found.

\section{Limitations}

This pilot study needs to be interpreted with caution. Firstly, a limited sample size was used which is mainly the result of the significant costs associated with $\mathrm{Cl}$ treatment. This emphasizes the importance to develop a cost-reducing alternative to a standard $\mathrm{Cl}$, specifically developed for tinnitus suppression. The used pattern generator shows that environmental sound is not specifically required to reduce tinnitus complaints and opens up future possibilities in this direction.

Secondly, the primary aim of this study was to investigate the effects of intracochlear electrical stimulation that does not encode environmental sounds on tinnitus [Arts et al., in press, 2015b, unpublished data]. The effects on speech reception were investigated using an explorative approach. This means that the current study was not specifically designed to answer the research questions posed in this article. That is, results may for example have been affected by confounding effects by tinnitus suppression, even though effort was undertaken to describe and investigate confounding effects adequately. However, using this explorative approach it is known that the used tinnitus specific stimulation significantly suppresses tinnitus [unpublished data].

Finally, no stratified randomization was used for the assignment of subjects to the groups with and without formal auditory training. Therefore, no control for ipsilateral and contralateral audiometric hearing thresholds was performed that could possibly bias the results on the effect of formal auditory training on speech perception using $\mathrm{Cl}$ in SSD. In future studies, we plan to specifically investigate the influence of meaningless intracochlear electrical stimulation developed for tinnitus suppression on speech perception.

In conclusion, the current clinical trial, including 10 patients with single-sided deafness, shows a statistically significant improvement after two months of standard $\mathrm{Cl}$ on speech reception in quiet, speech reception in noise and the subjective ability of hearing. Formal auditory training during $\mathrm{Cl}$ rehabilitation was not statistically advantageous on either of the hearing related outcomes. However, a statistically significant benefit was found in $\mathrm{Cl}$-related discomfort during $\mathrm{Cl}$ rehabilitation. Electrical stimulation that does not encode environmental sounds appears to be neither advantageous nor disadvantageous for speech perception. Furthermore, a statistically significant advantage of standard $\mathrm{Cl}$ was found on speech reception in quiet, speech reception in noise and the subjective ability of hearing, compared to electrical stimulation that does not encode environmental sounds.

\section{Acknowledgements:}

The authors are very grateful to the participants for their time and effort during data collection. Furthermore, the authors gratefully acknowledge the contribution from X.S. van der Tier from Maastricht $\mathrm{UMC}+$ for her valuable contribution in providing the formal auditory training. This paper is also in memoriam of our colleague Jogina Hettinga, who passed away on November 18, 2014. This study was financially supported by MED-EL (MED-EL Corporation, Innsbruck, Austria).

\section{Conflict of interest:}

This work was supported by a research grant from MED-EL Corporation, Innsbruck, Austria. The funding source had no involvement in the study design; in the collection, analyses and interpretation of data; in the writing of the report; or in the decision to submit the article for publication. The authors declare that they have no conflict of interest. 
References:

Arndt S, Aschendorff A, Laszig R, Beck R, Schild C, Kroeger S, Ihorst G, Wesarg T: Comparison of pseudobinaural hearing to real binaural rehabilitation after cochlear implantation in patients with unilateral deafness and tinnitus. Otol Neurotol 2010;32:39-47.

Arndt S, Laszig R, Aschendorff A, Beck R, Schild C, Hassepa $\beta$ F, Ihorst G, Kroeger S, Kirchem P, Wesarg T: Einseitige Taubheit und Cochleaimplantat-Versorgung. HNO 2011;59:437-446.

Arts RA, George EL, Chenault MN, Stokroos RJ: Optimizing intracochlear electrical stimulation to suppress tinnitus. Ear Hear 2015a; 36(1):125-35.

Arts RAGJ, George ELJ, Griessner A, Zierhofer C, Stokroos RJ: Long term effects of intracochlear electrical stimulation with looped patterns on tinnitus: a case report. ENT J, in press.

Arts RA, George EL, Griessner A, Zierhofer C, Stokroos RJ: Tinnitus suppression by intracochlear electrical stimulation in single sided deafness - a prospective clinical trial: Part I. Audiol Neurotol 2015b; 20:294-313.

Arts RA, George EL, Stokroos RJ, Vermeire K: Review: Cochlear implants as a treatment of tinnitus in single-sided deafness. Curr Opin Otolaryngol Head Neck Surg 2012;20:398-403.

Blasco MA, Redleaf MI: Cochlear implantation in unilateral sudden deafness improves tinnitus and speech comprehension: meta-analysis and systematic review. Otol Neurotol 2014 [Epub ahead of print].

Bosman AJ, Wouters J, Damman W: Realisatie van een cd voor spraakaudiometrie in Vlaanderen. Logopedie en Audiologie 1995;9:218-225.

Buechner A, Brendel M, Lesinski-Schiedat A, Wenzel G, Frohne-Buechner C, Jaeger B, Lenarz T: Cochlear implantation in unilateral deaf subjects associated with ipsilateral tinnitus. Otol Neurotol 2010;31:13811385.

Carhart R: Monaural and binaural discrimination against competing sentences. Intern Audiol 1965;4:510.

Chang JE, Zeng F-G: Tinnitus suppression by electric stimulation of the auditory nerve. Front Syst Neurosci DOI: 10.3389/2012/00019.

Chen JM, Shipp D, Al-Abidi A, Ng A, Nedzelski JM: Does choosing the "worse" ear for cochlea implantation affect outcome? Otol Neurotol 2001;22:335-339.

Cochlear Clinical background. Available at: http://cochlear-cee-training.org/wordpress/wp-content/ uploads/2011/11/E82075_-_Clinical_background_Single-sided_deafness_GB.pdf. Accessed April 8, 2015.

De Cassi a Cassou Guimarães Mendes R, Ribas A, De Macedo IP, Buschle M, Ataíde AL, Pereira R, Kochen AP: Cochlear implant in the treatment of incapacitating unilateral tinnitus: case report. Int Tinnitus J 2012;17(2):200-4.
Firszt JB, Holden LK, Reeder RM, Cowdrey L, King S: Cochlear implantation in adults with symmetrical hearing loss. Ear Hear 2012a;33(4):521-533.

Firszt JB, Holden LK, Reeder RM, Waltzman SB, Arndt S: Auditory abilities after cochlear implantation in adults with unilateral deafness: a pilot study. Otol Neurotol 2012b;33:1339-1346.

Gartrell BC, Jone

s HG, Kan A, Buhr-Lawler M, Gubbels SP, Litovsky RY: Investigating long-term effects of cochlear implantation in single-sided deafness: a best practice model for longitudinal assessment of spatia hearing abilities and tinnitus handicap. Otol Neurotol 2014;35:1525-1532.

Gatehouse S, Akeroyd M: Two-eared listening in dynamic situations. Int J Audiol 2006; 45(1):S120-S124 Gatehouse S, Noble W: The Speech, Spatial, and Qualities of Hearing Scale (SSQ). Int J Audiol 2004:43:85-99.

Grizzle JE: The two-period change-over design and its use in clinical trials. Biometrics, 21, 467-480. Hansen MR, Gantz BJ, Dunn C: Outcomes after cochlear implantation for patients with single-sided deafness, including those with recalcitrant Ménièr's disease. Otol Neurotol 2013;34:1681-1687. Hassepass F, Aschendorff A, Wesarg T, Kröger S, Laszig R, Beck RL, Schild C, Arndt S: Unilateral deafness in children: audiologic and subjective assessment of hearing ability after cochlear implantation. Otol Neurotol 2012;34:53-60

Jacob R, Stelzig Y, Nopp P, Schleich P: Audiologische Ergebnisse mit Cochleaimplantat bei einseitiger Taubheit. HNO 2011;59:453-460.

Kleine Punte A, Vermeire K, Hofkens A, De Bodt M, De Ridder D, Van de Heyning P Cochlear implantation as a durable tinnitus treatment in single-sided deafness. Cochlear Implants Int 2011:12(1):S26-9. Kleinjung T, Steffens T, Strutz J, Langguth B: Curing tinnitus with a cochlear implant in a patient with unilateral sudden deafness: a case report. Cases J 2009;2:7462

Kokkinakis K, Pak N: Binaural advantages in users of bimodal and bilateral cochlear implant devices. Acoust Soc Am 2014;135(1):EL47-53.

Lammers MJW, Van der Heijden GJMG, Pourier VEC, Grolman W: Bilateral cochlear implantation in children: a systematic review and best-evidence synthesis. Laryngoscope 2014;124:1694-1699.

Mertens G, Kleine Punte A, De Bodt M, Van de Heyning P: Binaural auditory outcomes in patients with postlingual profound unilateral hearing loss: 2 years after cochlear implantation. Audiol Neurotol 2015;20(1):67-72.

Mertens G, Kleine Punte A, De Ridder D, Van de Heyning P: Tinnitus in a single-sided deaf ear reduce speech reception in the nontinnitus ear. Otol Neurotol 2013;34:662-666.

National Institute on Deafness and Other Communication Disorders, National Institute of Health. Cochlear implants. Available at: http://www.nidcd.nih.gov/health/hearing/pages/coch.aspx. Accessed April 8, 2015. 
Nawaz S, McNeill C, Greenberg SL: Improving sound localization after cochlear implantation and auditory training for the management of single-sided deafness. Otol Neurotol 2014;35:271-276.

Olze H, Gräbel S, Haupt H, Förster U, Mazurek B: Extra benefit of a second cochlear implant with respect to health-related quality of life and tinnitus. Otol Neurotol 2012;33:1169-1175.

Palau EM, Gil JLM, Vidal CM, González JCF, Cabrera OA, Macías ÁR: Tinnitus and cochlear implantation. Preliminary experience. Acta Otorrinolaringol Esp 2010;61(6):405-411.

Plomp P, Mimpen AM: Improving the reliability of testing the speech perception thresholds for sentences. Audiology 1979;18:43-52.

Ramos Á, Polo R, Masgoret E, Artiles O, Lisner I, Zaballos ML, Moreno C, Osoria Á: Cochlear implant in patients with sudden unilateral sensorineural hearing loss and associated tinnitus. Acta Otorrinolaringol Esp 2012;63(1):15-20

Rubinstein JT, Tyler RS, Johnson A, Brown CJ: Electrical suppression of tinnitus with high-rate pulse trains. Otol Neurotol 2003;24:478-485.

Shaw EA: Transformation of sound pressure level from the free field to the eardrum in the horizontal plane. J Acoust Soc Am 1974;56:1848-1861.

Schleich P, Nopp P, D'Haese P: Head shadow, squelch, and summation effects in bilateral users of the MED-EL Combi 40/40+ cochlear implant. Ear Hear 2004;25(3):197-204.

Snik A, Agterberg M, Bosman A: How to quantify binaural hearing in patients with unilateral hearing using hearing implants. Audiol Neurotol 2015;20(1):44-47.

Stelzig Y, Jacob R, Mueller J: Preliminary speech recognition results after cochlear implantation in patients with unilateral hearing loss: a case series. Journal of Medical Case Reports 2011;5:343.

Távora-Vieira D, Marino R, Acharya A, Rajan GP: The impact of cochlear implantation on speech understanding, subjective hearing performance, and tinnitus perception in patients with unilateral severe to profound hearing loss. Otol Neurotol 2015;36(3):430-6.

Távora-Vieira D, Marino R, Krishnaswamy J, Kuthbutheen J, Rajan GP: Cochlear implantation for unilateral deafness with and without tinnitus: a case series. Laryngoscope 2013;123(5):1251-5.

Tokita J, Dunn C, Hansen MR: Cochlear implantation and single-sided deafness. Curr Opin Otolaryngo Head Neck Surg 2014;22(5):353-8.

Van de Heyning P, Vermeire K, Diebl M, Nopp P, Anderson I, De Ridder D: Incapacitating unilateral tinnitus in single-sided deafness treated by cochlear implantation. Ann Oto Rhinol Laryn 2008;117(9):645-652. van Schoonhoven J, Sparreboom M, van Zanten BG, Scholten RJ, Mylanus EA, Dreschler WA, Grolman W, Maat $B$ : The effectiveness of bilateral cochlear implants for severe-to-profound deafness in adults: a systematic review. Otol Neurotol 2013;34:190-198.

Van Wieringen A, Wouters J: LIST and LINT: sentences and numbers for quantifying speech understanding in severely impaired listeners for Flanders and The Netherlands. Int J Audio 2008;47:348-255.

Van Zon A, Peters JPM, Stegeman I, Smit AL, Grolman W: Cochlear implantation for patients with singlesided deafness or asymmetrical hearing loss: a systematic review of the evidence. Otol Neuroto 2015;36:209-219.

Vermeire K, Van de Heyning P: Binaural hearing after cochlear implantation in subjects with unilatera sensorineural deafness and tinnitus. Audiol Neurotol 2009:14:163-171.

Vernon JA, Meikle MB: Masking devices and alprazolam treatment for tinnitus. Otolaryngol Clin N Am 2003;36:307-320

Vlastarakos PV, Nazos K, Tavoulari E-F, Nikolopoulos TP: Cochlear implantation for single-sided deafness: the outcomes. An evidence-based approach. Eur Arch Otorhinolaryngol 2014;271(8):2119-26.

Zeng F-G, Tang Q, Dimitrijevic A, Starr A, Larky J, Blevins NH: Tinnitus suppression by low-rate electric stimulation and its electrophysiological mechanisms. Hear Res 2011;277:61-66. 
The general objective of this thesis was to evaluate the effectiveness of intracochlear electrical stimulation on tinnitus and optimize stimulation characteristics for tinnitus suppression. Chapter 1 is a review article while Chapter 2 to 7 are original work. Of the original work, Chapter 2 reports on a retrospective study while Chapter 3 to 7 report on prospective studies. Chapter 4 to 7 were based on data from a cohort of single sided deaf subjects with tinnitus localized to the deaf ear.

\section{Summary of findings}

In Chapter 1, we performed the first (scoping) review that reveals an overview of all publicly available reports about $\mathrm{Cl}$ use as a treatment for tinnitus in SSD. Here, nine full research articles have been reported. We found that $\mathrm{Cl}$ suppresses tinnitus in most of the single sided deaf patients. Some studies even demonstrate complete tinnitus suppression after $\mathrm{Cl}$-activation. No tinnitus worsening is reported in any of the cases. The tinnitus level seems to stabilize after 3-6 months after the first fitting. In conclusion, cochlear implantation should be considered as a treatment option for tinnitus in SSD. However, appropriate patient selection seems to be essential for the effectiveness as it is expected that tinnitus arising from cochlear deafferentiation is a requirement for a positive effect.

However, induction of tinnitus is also a known complication of $\mathrm{Cl}$-surgery, although the incidence varies considerably among the studies. To date, it remains unclear why some $\mathrm{Cl}$-recipients experience tinnitus complaints due to $\mathrm{Cl}$-surgery while others do not. A possible explanation is that deterioration of residual hearing as a result of the traumatic insertion of the electrode array may trigger the experience of tinnitus postoperatively.

Chapter 2 describes a retrospective study using the database of the Cl-team of South-East Netherlands, based at the Maastricht University Medical Center. Of the 512 patients who were implanted between 2003 and 2013, 247 patients met the inclusion criteria and were sent a questionnaire. Of these, 197 patients were included. No statistically significant association between perioperative deterioration of hearing thresholds measured by pure tone audiometry and the development or deterioration of tinnitus was found. In conclusion, for patients with preoperative residual hearing, there appears to be no increased risk of developing chronic tinnitus after hearing deterioration due to cochlear implantation. Since $\mathrm{Cl}$ seems to have a positive effect on tinnitus (Chapter 1), the question arises what mechanism is responsible for tinnitus suppression after cochlear implantation. Is this suppression due to the reversal of the assumed maladaptive plasticity or is it simply the shift in attention from the tinnitus to environmental sound and therefore less awareness that reduces tinnitus perception?

In Chapter 3, a prospective clinical trial is reported about a cohort of eleven subjects with bilateral severe to profound hearing loss, unilateral $\mathrm{Cl}$ and tinnitus. The majority of the subjects were able to experience short-term tinnitus reduction through intracochlear electrical stimulation independent of acoustic sounds. Tinnitus can be reduced with audible or even inaudible, subthreshold stimuli. No clear trends were found in optimal stimulation characteristics. Optimal stimulus characteristics for tinnitus reduction therefore appear to be highly subject-specific.

In order to investigate the long-term effects of intracochlear electrical stimulation independent of acoustic input, a contralateral normal hearing ear is required. This contralateral ear enables the communication during intracochlear electrical stimulation independent of acoustic sound. 
Chapter 4 reports therefore about a case presented with a two-year history of debilitating tinnitus in the right ear following an idiopathic profound sudden sensorineural hearing loss in this right ear with a Pure Tone Average (PTA; averaged across 0.5, 1 and $2 \mathrm{kHz}$ ) of $97 \mathrm{~dB}$ Hearing Level (HL). His right ear was implanted with a $\mathrm{Cl}$ in order to suppress his tinnitus. The standard clinical $\mathrm{Cl}$ rehabilitation was followed by a three-month period of looped intracochlear electrical stimulation (without environmental sound perception). Tinnitus can be reduced for months with both, standard clinical $\mathrm{Cl}$ and intracochlear electrical stimulation independent of an acoustic input. No negative effect of intracochlear electrical stimulation independent of an acoustic input on speech perception in noise was observed. Furthermore, the additional use of a clinical $\mathrm{Cl}$ is advantageous for speech discrimination in the reported SSD subject.

Although the results of Chapter 4 are promising, the evidence of this finding is limited since a single case is reported. Therefore, a cohort of ten single sided deaf subjects with tinnitus localized to the deaf ear is described in Chapter 5-7.

Chapter 5 describes a prospective clinical trial about the short term effects of looped electrical stimulation on tinnitus (without environmental sound perception). Furthermore, with the used study design we were able to answer three additional research questions: 1. Does the surgery itself suppress tinnitus? 2. Does the clinical $\mathrm{Cl}$ suppress tinnitus? and 3 . Is a one-week period of Cl-deactivation sufficient to rule out the possible influence of providing a hearing sensation on the experience of tinnitus? The results suggest that tinnitus can be reduced with looped electrical stimulation, in some cases even with inaudible stimuli. The optimal stimuli for tinnitus suppression appear to be subject-specific. However, medium-to-loud stimuli suppress tinnitus significantly better than soft stimuli which can partly be explained by the masking effect. Furthermore, this chapter shows a tinnitus reduction during standard clinical $\mathrm{Cl}$ rehabilitation while the $\mathrm{Cl}$ surgery itself had no positive or negative effect on tinnitus. The baseline (original) tinnitus loudness was restored after one week of $\mathrm{Cl}$-deactivation. These findings corroborate to the hypothesis that tinnitus is related to unwanted neuroplasticity related to hearing loss, and indicate that tinnitus can be suppressed at least for minutes by looped intracochlear electrical stimulation.

The main objective of Chapter 6 is to investigate the possibility for the development of a "Tinnitus Implant" (TI), an intracochlear pulse generator for the suppression of tinnitus. Although the results obtained in Chapter 5 seem to be promising, long-term tinnitus suppression is a requirement for the viability of the TI. Therefore, long-term suppressive effects of looped electrical stimulation (without environmental sound perception) were compared with the standard stimulation pattern of a $\mathrm{Cl}$ (with environmental sound perception). This chapter shows that tinnitus can be suppressed with intracochlear electrical stimulation independent of environmental sounds, even long-term. No statistically significant difference in tinnitus suppression was found between the standard clinical $\mathrm{Cl}$ and the experimental TI.

Coding of environmental sounds seems to be no requirement for tinnitus suppression using intracochlear electrical stimulation. Furthermore, it appears to be possible to suppress tinnitus using inaudible stimuli. It is therefore plausible that tinnitus suppression by $\mathrm{Cl}$ is not solely caused by an attention shift from the tinnitus to environmental sounds. Both the standard clinical $\mathrm{Cl}$ and the experimental $\mathrm{Tl}$ are potential treatment options for tinnitus. Nonetheless, a last relevant research question remains still unanswered, namely: What is the effect of intracochlear electrical stimulation that does not encode environmental sounds, but suppresses tinnitus, on speech perception in SSD?

In Chapter 7, the effect of looped electrical stimulation on speech reception was reported. Furthermore we answered two additional research questions: 1 . What is the effect of the standard clinical $\mathrm{Cl}$ on speech reception in SSD? and 2. Is formal auditory training during CI-rehabilitation in SSD beneficial?
The results suggest that tinnitus specific electrical stimulation has neither positive nor negative effects on speech reception. Standard clinical CI in SSD is beneficial in speech perception in quiet, speech reception in noise and the subjective ability of hearing. Furthermore, formal auditory training seems not to be beneficial in speech reception. However, Cl-related discomfort significantly reduced more rapidly during $\mathrm{Cl}$ rehabilitation in subjects receiving formal auditory training.

\section{General discussion}

\section{Methodological considerations}

This thesis is characterized by the lack of objective outcome measures which is unavoidable in case of subjective tinnitus. As tinnitus is a subjective symptom, researchers are dependent on the collaboration and honesty of their subjects. In Chapter 5 and 6 validated and widely used questionaires were used: the Tinnitus Hanidicap Inventory [Newman et al., 1996; Zeman et al., 2012] and the Tinnitus Questionnaire [Hallam et al., 1988; Meeus et al., 2007]. Athough these questionnaires have proven useful for measuring individual differences regarding tinnitus severity, an important limitation is that these questionnaires have not been validated to measure effectiveness of interventions. They were therefore not designed to maximize responsiveness (i.e., sensitivity for measuring treatment-related changes in tinnitus). However, at the time when the protocol was developed, none of the available tinnitus questionnaires was validated to measure effectiveness of interventions. For future interventional studies, the newly developed Tinnitus Functional Index [Meikle et al., 2012] or Tinnitus Primary Function Questionnaire [Tyler et al., 2014] are suggested.

Dobie [1999] found that the tinnitus population is highly sensitive to placebo effects. Therefore, all intervention studies for tinnitus suppression (Chapter 3-6) implemented a single blinded sham condition to control for placebo effects. Although not completely free from bias, a single blinded approach was chosen for practical reasons. The implementation of a placebo condition is exceptional in tinnitus research with $\mathrm{Cl}$ and only possible in studies using (additional) electrical stimulation which is independent of environmental sounds.

No subjects dropped out prematurely in our clinical trial using single sided deaf subjects (Chapter 4-7). However, in our clinical trial using subjects with bilateral severe to profound hearing loss (Chapter 3), two subjects dropped out prematurely. As one of these subjects dropped out due to a positive reason; a residual inhibition (RI) of more than half an hour while the other subject dropped out because of a more negative reason; fear of possible side effects, we concluded that there was no selective drop-out which is a known potential source of selection bias.

Confounding occurs when the effect of a variable of interest is affected or blurred by that of a third variable [Grimes and Schulz, 2002]. To overcome this bias, we checked for multiple potential confounders in Chapter 2, which makes it unlikely that residual confounding has influenced the results. Unfortunately, due to the limited sample sizes we were not able to check for potential confounders of tinnitus suppression in our prospective clinical trials (Chapter 3,5-6). During the trials we avoided any form of cognitive behavioral therapy as this management treatment has a known positive effect on tinnitus distress [Cima et al., 2012]. Nevertheless, for clinical implications a combination of both treatment options might be favourable. 
In Chaper 5 of this thesis we conclude that the $\mathrm{Cl}$-surgery itself has no positive or negative effect on tinnitus in the studied cohort. In Chapter 6 a statistically significant tinnitus reduction was observed at the end of the follow-up compared to baseline while no effect on tinnitus was observed in the sham condition. A similar effect was found in all tinnitus outcome measurements used (Chapter 5 and 6 ). It seems therefore likely that intracochlear electrical stimulation has a causal relation to tinnitus suppression. Nevertheless, the major methodological concern is the limited sample sizes in our prospective clinical trials (Chapter 3, 5-7). The limited sample size in Chapter 5-7 is mainly caused by the substantial costs associated with this treatment option. This emphasizes the importance of our research. Here, a potential cheaper treatment option compared to the conventional $\mathrm{Cl}$, including a highly sophisticated speech processing strategy, was reported to be effective. A relatively simple pattern generator is sufficient for this treatment option. This pilot study was designed as a feasibility study which opens new possibilities for the development of a "Tinnitus Implant" (TI); a tinnitus specific $\mathrm{Cl}$, and therefore future research is highly recommended.

\section{Future directions}

The results obtained from Chapter 4-6 on tinnitus suppression and Chapter 7 on speech reception using standard clinical $\mathrm{Cl}$ could possibly be further optimized in future studies. Postoperative imaging using $\mathrm{x}$-rays improves for example the knowledge about the position of the individual electrodes and prevent a possible mismatch between the default frequency allocation table in the standard clinical software [Landsberger et al., 2015] and the predicted frequency allocation based on the available frequencyposition functions [Greenwood, 1990; Stakhovskaya et al., 2007]. A three dimensional image analysis of the cochlear electrode position could possibly optimize the predicted frequency allocation of the electrodes inserted [Gazibegovic et al., 2014].

Although no evidence was found for tinnitus pitch matched stimulation as the optimal stimulation characteristic for intracochlear electrical stimulation to suppress tinnitus (Chapter 3 and 5), this treatment option could possibly be optimized by Magnetic Resonance Imaging (MRI). Pre-operative ultra-high field (functional) MRI of the central nervous system could plausibly show more accurate and objective information about the optimal stimulation site inside the cochlea for tinnitus suppression compared to the subjective tinnitus pitch matching procedure used in Chapter 3-5. The tonotopic organization of the cortical [Moerel et al., 2014] and subcortical [De Martino et al., 2013] structures of the auditory pathway can be used to predict the optimal stimulation site inside the cochlea. By means of post-operative Computed Tomography (CT), the exact position of the individual electrodes can be determined [Gazibegovic et al., 2014] and can be calculated which electrode corresponds to the best position of the stimulation.

Chapter 6 of this thesis suggests that intracochlear electrical stimulation for tinnitus suppression does not require acoustic information. Furthermore, hearing seems to be able, due to less traumatic surgery [Van Abel et al., 2015], to be preserved to some extent. Future research on the effect of meaningless intracochlear electrical stimulation on tinnitus could therefore possibly be extended to a population with significant (ipsilateral) residual hearing. Alternatively, extracochlear stimulation [Di Nardo et al., 2009] for tinnitus suppression seems to be a potential treatment option in a selection of tinnitus patients whereby hearing preservation is desirable. Moreover, it might be possible that the effectiveness of intracochlear electricalstimulation on tinnitussuppression could befurtheroptimized using a combination of speech processing and the meaningless, tinnitus specific, intracochlear electrical stimulation [Tyler et al., 2015].
Chapter 3-6 of this thesis suggest that the observed tinnitus suppression is brought about by the reversal of the assumed maladaptive plasticity and is not simply due to a shift in attention from the tinnitus to environmental sound and therefore less awareness that reduces tinnitus perception. Both the frequently observed residual inhibition and the ability to reduce tinnitus with inaudible (subthreshold stimulation or incase of loudness adaptation) electrical stimulation support this hypothesis. This suggested physiological effect together with the plausible heterogeneity in the cause of tinnitus makes neurostimulation of the vestibulocochlear nerve [Bartels et al., 2007], subcortical deep brain stimulation [Smit et al., 2015], and cortical stimulation [Engelhardt et al., 2014] potential treatment options for tinnitus. However, intracochlear electrical stimulation seems to be preferable because of the relative simplicity to access the structure of interest, the relatively simple course of cochlear's tonotopic organization [Greenwood, 1990] and the fact that the cochlea is at the seed of the auditory neural afferent pathway were the information is not forwarded yet to both sides (ipsilateral and contralateral) of the brain stem, and later, both hemispheres. Furthermore, the central nervous system is more complicated, and the connections are less well understood, because there are multiple parallel pathways.

Although intracochlear electrical stimulation seems to be a viable treatment option for tinnitus, a customized treatment is required for clinical implications, and therefore one should consider all available potential treatment options, starting with the least invasive treatment option like conventional hearing aids [Hoare et al., 2014], sound enrichment using music with tinnitus related modification [Tass et al., 2012] and/or cognitive behaviour therapy [Jastreboff 1999; Cima et al., 2012].

Nevertheless, prevention remains the most efficient way to reduce the prevalence of tinnitus and therefore stress [Mazurek et al., 2015], acoustic trauma and ototoxic drugs (e.g. some analgesics, antibiotics, antineoplastics, antimalarials, loop diuretics and Valproate) which could induce tinnitus should be avoided [Zimmerman and Timboe, 2014]. Preventive campaigns such as educational programs on the understanding of noise-induced symptoms to prevent permanent hearing loss and tinnitus due to recreational noise exposure by the use of hearing protection are recommended [Gilles et al., 2013]. 


\section{Valorization addendum}

Although the results obtained in these feasibility studies are promising, one could doubt whether patients would accept such an invasive and expensive procedure to treat their tinnitus.

\section{Treatment acceptance}

To reduce tinnitus completely, $38 \%$ of the 197 tinnitus sufferers who attended a meeting of the Australian Tinnitus Society would accept (i.e., they indicated an acceptance of $91-100 \%$ ) a $\mathrm{Cl}$ as treatment option while $25 \%$ would accept a $\mathrm{Cl}$ in case of reduced tinnitus loudness and annoyance by half. This acceptance rate is comparible to noninvasive wearable devices (42\% and $30 \%$, respectively) [Tyler, 2012]. In contrast, based on a Dutch online survey on 415 tinnitus sufferers and member of the Dutch society for the hearing impaired, $29 \%$ of the population express a large interest in $\mathrm{Cl}$ (i.e., they scored their acceptance $8-10$ on visual analogue scale from 0 to 10 ) in case there is a $100 \%$ chance of full tinnitus suppression while $22 \%$ is very interested in case there is a $50 \%$ chance of full tinnitus suppression. A decrease in treatment acceptability was observed compared to the noninvasive conventional hearing aids, with an acceptance rate of $53 \%$ and $51 \%$ respectively (unpublished data). The difference in $\mathrm{Cl}$-acceptance in relation to noninvasive wearable devices between both studies can probably be explained by selection bias. Nevertheless, both studies have shown that the acceptance of cochlear implantation as a treatment option for tinnitus is considerable which is consistent with the results obtained in an American internet survey with 439 responders. Here, almost three-fourths of the patients would be willing to have a device implanted in their body for a therapy that could eliminate or reduce their tinnitus perception by half [Engineer et al., 2013].

\section{Economic consideration}

As mentioned in the introduction of this thesis, tinnitus affects about 50 million people in the United States and an estimated 70 million in the European Union [Cederroth et al., 2013] which is about 10-15\% of the general population [Langguth et al., 2013]. The estimated tinnitus prevalence in the Netherlands is 2 million of whom about 60.000 severely suffer from their tinnitus [Cima et al. 2009]. Furthermore, Maes and colleagues [2013] concluded that the economic burden of tinnitus to society is substantial with an on average annual tinnitus related societal cost per patient of $€ 5,315$ in the Netherlands. Most of these costs were associated with production losses; on average $€ 3,702$. Overall, the annual costs of tinnitus in the Netherlands examined from a societal perspective is about $€ 6.8$ billion (95\% confidence interval: $€ 3.9$ billion - $€ 10.8$ billion). The annual health care costs were $€ 1.9$ billion ( $95 \%$ confidence interval: $€ 1.4$ billion $€ 2.5$ billion) which amounts to $2.3 \%$ of the total Dutch health care expenditure [Maes et al., 2013].

Based on these resultsitisstriking that within the National Institute on Deafness and other Communication Disorders, tinnitus funding accounts for only $2 \%$ (about $\$ 5$ million per year from 2009 to 2011) of total hearing research funds [Cederroth et al., 2013]. Private donors like for example the founder of the Tinnitus Research Initiative; Matteo de Nora, the fundraising efforts of for example the American Tinnitus Association and investments from the industry are therefore essential for the knowledge about and treatment options for tinnitus.

Moreover, Tyler [2012] reported about the willingness to pay for tinnitus treatments and asked 197 tinnitus sufferers to indicate how much money they would be willing to pay for effective treatments. Most of these patients indicated to pay at least $\$ 5000$ and $20.3 \%$ were willing to pay as much as $\$ 25,000$ for complete tinnitus reduction. Furthermore, Engineer and colleagues [2013] reported about an online survey with 439 responders of which $94 \%$ reported that they had health insurence. Here, almost $40 \%$ had already spent between $\$ 500$ and $\$ 10,000$ on tinnitus therapies. Therefore, from an economical point of view, the tinnitus population is an interesting market for the industry with in particular the industry of medical devices as novel drugs take a long time and hundreds of millions of dollars to develop. For purposes of illustration, Auris Medical recently invested $\$ 51$ million for a phase 3 trial in tinnitus for the pharmacotherapy using AM-101 [Cederroth et al., 2013]. In contrast, the average implantable medical device takes about $\$ 10$ million and five years to develop [Engineer et al., 2013]

This research project is funded by one of our $\mathrm{Cl}$ industry partners. They are interested in the long-term effects of electrical stimulation independent of environmental sounds on tinnitus as they see new opportunities to expand their market. Although the highly sophisticated $\mathrm{Cl}$ shows promising results concerning the tinnitus suppression, the considerable costs (about $€ 25,000$ for the device [Broersen 2010]) have hampered the clinical application. With the promising long-term effects of electrical stimulation independent of environmental sounds on tinnitus, a "Tinnitus Implant" (TI) should by viable. This relatively simple neurostimulator could be produced considerably cheaper because it does not require a strategy to encode environmental sounds. This thesis includes feasibility studies (Chapter 3-7) which open new possibilities for the development of this treatment option.

\section{Possible effects on the standard clinical Cl}

The results obtained on tinnitus suppression may indirectly lead to future research in order to expand the inclusion criteria for $\mathrm{Cl}$, especially regarding residual hearing. To date, there is a considerable risk of damaged residual hearing due to the implantation itself, which is the main reason why only subjects who were deaf in the ear to be implanted were included. Changes in both the device and the surgical procedure could reduce this risk. If this risk can be neglected, this treatment option could possibly be suitable for the tinnitus population with significant residual hearing. This thesis can contribute to the interest to develop an atraumatic procedure for electrode insertion. Although Chapter 2 of this thesis seems to suggest that deterioration of residual hearing as a result of the implantation itself does not cause postoperative chronic tinnitus, hearing loss as a side effect of the electrode insertion for tinnitus treatment is undesirable.

For safety as well as methodological reasons the long-term effects of intracochlear electrical stimulation independent of environmental sounds were investigated using the standard clinical $\mathrm{Cl}$ which was reprogrammed to function as a tinnitus specific neurostimulator. This gave us the opportunity to additionally investigate the audiological effects of the standard Cl in the SSD population (Chapter 7). The positive effects of $\mathrm{Cl}$ obtained can contribute to the broadening of the indication for the standard clinical $\mathrm{Cl}$. For example, in Belgium and Germany the indication for $\mathrm{Cl}$ is expanded already to SSD associated with tinnitus (the $\mathrm{Cl}$ system is CE-Market for SSD in both children and adults), because in these countries the value of $\mathrm{Cl}$ in SSD has been proven [Van de Heyning et al., 2008; Kleinjung et al., 2009; Vermeire et al. 2009; Arndt et al., 2010; Buechner et al., 2010; Arndt et al., 2011; Jacob et al., 2011; Stelzig et al., 2011 Hassepass et al., 2012; Mertens et al., 2013]. It is plausible that the Dutch policy makers (College voor Zorgverzekeringen) will follow after research in the Netherlands has reproduced these findings.

\section{Knowledge valorization}

The findings and ideas have been communicated to and shared with fellow researchers around the world at (inter)national congresses and in scientific journals. Furthermore, a huge appreciation for our work is observed in the tinnitus population. The Maastricht UMC+ organizes for example every six months tinnitus symposium in collaboration with the Dutch society for the hearing impaired (Nederlandse Vereniging Voor Slechthorenden). These symposia were always fully booked. Here, background 
information was given about tinnitus and hearing loss and recent findings from research projects were shared. Frequent interest of national media resulted in a generally better understanding of tinnitus. As tinnitus remains refractory to current medical treatment, sufferers are regularly disappointed after consulting a physician and do often not feel taken seriously. This clinical research contributes to the patient's awareness of global developments in this field of research.

\section{References}

Arndt S, Aschendorff A, Laszig R, Beck R, Schild C, Kroeger S, lhorst G, Wesarg T: Comparison of pseudobinaural hearing to real binaural hearing rehabilitation after cochlear implantation in patients with unilateral deafness and tinnitus. Otol Neurotol 2010;32:39-47.

Arndt S, Laszig R, Aschendorff A, Beck R, Schild C, Hassepaß F, Ihorst G, Kroeger S, Kirchem P, Wesarg T: Einseitige Taubheit und Cochleaimplantat-Versorgung. HNO 2011;59:437-446.

Bartels $\mathrm{H}$, Staal MJ, Holm AF, Mooij JJ, Alberts FW: Long-term evaluation of treatment of chronic, therapeutically refractory tinnitus by neurostimulation. Stereotact Funct Neurosurg 2007;85(4):150-7.

Broersen SFM: Cochleair implantaat opent de wereld. Medisch Contact 2010;10:528-531.

Buechner A, Brendel M, Lesinski-Schiedat A, Wenzel G, Frohne-Buechner C, Jaeger B, Lenarz T: Cochlear implantation in unilateral deaf subjects associated with ipsilateral tinnitus. Otol Neurotol 2010;31:1381 1385.

Cederroth CR, Canlon B, Langguth: Hearing loss and tinnitus - are funders and industry listening? Nature Biotechnology 2013;31(11):972-4.

Cima RF, Maes IH, Joore MA, Scheyen DJ, El Refaie A, Baguley DM, Anteunis LJ, van Breukelen GJ, Vlaeyen JW: Specialised treatment based on cognitive behaviour therapy versus usual care for tinnitus: a randomized controlled trial. Lancet 2012;379(9830):1951-9.

De Martino F, Moerel M, van de Moortele PF, Ugurbil K, Goebel R, Yacoub E, Formisano E: Spatial organization of frequency preference and selectivity in the human inferior colliculus. Nat Commun 2013;4:1386.

Di Nardo W, Cianfrone F, Scorpecci A, Cantone I, Giannantonio S, Paludetti G: Transtympanic electrical stimulation for immediate and long-term tinnitus suppression. Int Tinnitus J 2009;15(1):100-6.

Dobie RA: A review of randomized clinical trials in tinnitus. Laryngoscope 1999;109:1202-1211.

Engelhardt J, Dauman R, Arné P, Allard M, Dauman N, Branchard O, Perez P, Germain C, Caire F, Bonnard D, Cuny $E$ : Effect of chronic cortical stimulation on chronic severe tinnitus: a prospective randomized double-blind cross-over trial and long-term follow up. Brain Stimul 2014;7(5):694-700.

Engineer ND, Rosellini WM, Tyler RS: Willingness to accept and pay for implantable tinnitus treatments: a survey. Neuromodulation 2013;16(2):154-62.

Gazibegovic D, Dees G, van Hoof M, Stokroos R: The assessment of the scalar positionof the HiFocus Mis Scala electrode based on MRI and Cone Beam CT fusion. $8^{\text {th }}$ International symposium on objective measures in auditory implants 2014; http://www.epostersonline.com/omai2014/node/202

Gilles A, Van Hal G, De Ridder D, Wouters K, Van de Heyning P: Epidemiology of noise-induced tinnitus and the attitudes and beliefs towards noise and hearing protection in adolescence. PLOS ONE DOI: 10.1371/journal.pone.0070297. 
Greenwood DD: A cochlear frequency-position function for several species -29 years later. J Acoust Soc Am 1990;87(6):2592-2605.

Grimes DA, Schulz KF. Bias and causal associations in observational research. Lancet 2002;359:248-52.

Hassepass F, Aschendorff A, Wesarg T, Kröger S, Laszig R, Beck RL, Schild C, Arndt S: Unilateral deafness in children: audiologic and subjective assessment of hearing ability after cochlear implantation. Otol Neurotol 2012;34:53-60

Hallam RS, Jakes JC, Hinchcliffe R: Cognitive variables in tinnitus annoyance. Br J Clin Psychol $1988 ; 27: 213-222$

Hoare DJ, Edmondson-Jones M, Sereda M, Akeroyd MA, Hall D: Amplification with hearing aids for patients with tinnitus and co-existing hearing loss. Cochrane Database of Systematic Reviews DOI: 10.1002/14651858.CD010151.pub2.

Jacob R, Stelzig Y, Nopp P, Schleich P: Audiologische Ergebnisse mit Cochleaimplantat bei einseitiger Taubheit. HNO 2011;59:453-460.

Jastreboff PJ: Tinnitus retraining therapy. Br J Audiol 1999;33(1):68-70.

Kleinjung T, Steffens T, Strutz J, Langguth B: Curing tinnitus with a cochlear implant in a patient with unilateral sudden deafness: a case report. Cases J 2009;2:7462.

Landsberger DM, Svrakic M, Roland JT, Svirsky M: The relationship between insertion angles, default frequency allocations, and spiral ganglion place pitch in cochlear implants. Ear Hear 2015; [Epub ahead of print].

Langguth B, Kreuzer PM, Kleinjung T, De Ridder D: Tinnitus: causes and clinical management. Lancet Neurol 2013;12:920-30.

Maes IHL, Cima RFF, Vlaeyen JW, Anteunis LJC, Joore MA: Tinnitus: a cost study. Ear Hear 2013;34(4);508-514.

Mazurek B, Szczepek AJ, Hebert S: Stress and tinnitus. HNO 2015;63:258-265.

Meeus O, Blaivie C, Van de Heyning P: Validation of the Dutch and the French version of the Tinnitus Questionnaire. B-ENT 2007;3(Suppl 7):11-17.

Meikle MB, Henry JA, Griest SE, Stewart BJ, Abrams HB, McArdle R, Myers PJ, Newman CW, Sandridge S, Turk DC, Folmer RL, Frederick EJ, House JW, Jacobson GP, Kinney SE, Martin WH, Nagler SM, Reich GE, Searchfield G, Sweetow R, Vernon JA: The Tinnitus Functional Index: development of a new clinical measure for chronic, intrusive tinnitus. Ear Hear 2012;33(2):153-76.

Mertens G, Kleine Punte A, De Ridder D, Van de Heyning P: Tinnitus in a single-sided deaf ear reduces speech reception in the nontinnitus ear. Otol Neurotol 2013;34:662-666.
Moerel M, De Martino F, Formisano E: An anatomical and functional topography of human auditory cortical areas. Front Neurosci 2014;8:225

Newman CW, Jacobson GP, Spitzer JB: Development of the Tinnitus Handicap Inventory. Arch Otolaryngol Head Neck Surg 1996;122:143-148.

Smit JV, Janssen ML, Schulze H, Jahanshahi A, Van Overbeeke JJ, Temel Y, Stokroos RJ: Deep brain stimulation in tinnitus: current and future perspectives. Brain Res 2015;1608:51-65.

Stakhovskaya O, Sridhar D, Bonham BH, Leake P: Frequency map for the human cochlear spiral ganglion: implications for cochlear implants. JARO 2007;8:220-233.

Stelzig Y, Jacob R, Mueller J: Preliminary speech recognition results after cochlear implantation in patients with unilateral hearing loss: a case series. Journal of Medical Case Reports 2011:5:343.

Tass PA, Adamchic I, Freund H-J, von Stackelberg T, Hauptmann C: Counteracting tinnitus by acoustic coordinated reset neuromodulation. Restor Neurol Neurosci 2012;30:137-159.

Tyler RS: Patients preferences and willingness to pay for tinnitus treatments. J Am Acad Audio 2012;23:115-125

Tyler RS, Ji H, Perreau A, Witt S, Noble W, Coelho C: Development and validation of the tinnitus primary function questionnaire. Am J Audiol 2014;23(3):260-72.

Tyler RS, Keiner AJ, Walker K, Deshpande AK, Witt S, Killian M, Ji H, Patrick J, Dillier N, van Dijk P, Lai WK, Hansen MR, Gantz B: A series of case studies of tinnitus suppression with mixed background stimuli in a cochlear implant. Am J Audiol 2015; [Epub ahead of print].

Van Abel KM, Dunn CC, Sladen DP, Oleson JJ, Beatty CW, Neff BA, Hansen M, Gantz BJ, Driscoll CL: Hearing preservation among patients undergoing cochlear implantation. Otol Neurotol 2015;36(3):416-

Van de Heyning P, Vermeire K, Diebl M, Nopp P, Anderson I, De Ridder D: Incapacitating unilateral tinnitus in single-sided deafness treated by cochlear implantation. Ann Oto Rhinol Laryn 2008;117(9):645-652

Vermeire K, Van de Heyning P: Binaural hearing after cochlear implantation in subjects with unilateral sensorineural deafness and tinnitus. Audiol Neurotol 2009;14:163-171.

Zeman F, Koller M, Schecklmann M, Langguth B, Landgrebe M: Tinnitus assessment by means of standardized self-report questionnaires: Psychometric properties of the Tinnitus Questionnaire (TO) the Tinnitus Handicap Inventory (THI), and their short versions in an international and multi-lingual sample. Health and Quality of Life Outcomes DOI: 10.1186/1477-7525-10-128.

Zimmerman E, Timboe A: Tinnitus: steps to take, drugs to avoid. J Fam Pract 2014;63(2):82-8. 
Tinnitus (aurium), ook wel bekend als oorsuizen, is de betekenisloze fantoomsensatie van geluid. Ongeveer 50 miljoen mensen in de Verenigde Staten en een geschatte 70 miljoen in de Europese Unie ervaren tinnitus [Cederroth et al., 2013]. De geschatte prevalentie van tinnitus in Nederland is 2 miljoen waarvan ongeveer 60.000 mensen ernstig lijden aan tinnitus [Cima et al. 2009]. Sommige van hen ervaren hierdoor serieuze problemen in het dagelijks leven en overwegen zelfdoding [Turner et al., 2007]. Tot op heden blijft tinnitus resistent voor de huidige medische behandelopties en kan er enkel therapie worden aangeboden om te leren omgaan met tinnitus [Jastreboff 1999; Cima et al., 2012].

Hoewel de precieze oorzaak van tinnitus nog niet geheel duidelijk is, is de huidige consensus dat tinnitus het resultaat is van ongewenste plasticiteit in de centrale auditieve zenuwbanen als gevolg van auditieve deprivatie [Du Verney, 1731; Engineer et al., 2011]. Deze neurale plasticiteit als onderliggende oorzaak, en daarmee de tinnitus waarneming zou omkeerbaar moeten zijn met verhoogde auditieve stimulatie. Akoestische geluidsverrijking door zowel conventionele hoortoestellen [Saltzman and Ersner, 1947; Shekhawat et al., 2013; Hoare et al., 2014], tinnitus maskeerders [Erlandsson et al., 1987; Vernon and Meikle, 2003; Fioretti et al., 2012] of muziek met tinnitus-gerelateerde aanpassingen [Davis et al., 2007; Tass et al., 2012; Pantev et al., 2012a; Pantev et al., 2012b; Reavis et al., 2012] is een potentiële behandeloptie, maar laat een grote variabiliteit in effectiviteit tussen patiënten zien. Deze variabiliteit kan worden verklaard door de heterogeniteit van tinnitus dat lijkt te worden veroorzaakt door de auditieve deprivatie dat op verschillende niveaus van de auditieve signaalverwerking kan optreden. Omdat de meest voorkomende oorzaak van auditieve deprivatie in het slakkenhuis is gelegen ter hoogte van de haarcellen, dienen behandelopties deze pathologie te omzeilen.

Een mogelijkheid om deze haarcellen te omzeilen is het gebruik van een cochleair implantaat (CI). Een C is een elektronisch apparaat dat gedeeltelijk wordt geïmplanteerd in de cochlea (slakkenhuis). Het zet akoestisch geluid om in elektrische stroom dat de auditieve zenuw direct stimuleert. Het apparaat werd ontwikkeld voor patiënten met bilateraal ernstig tot zeer ernstig perceptief gehoorverlies waarbij geluidsversterking met een conventioneel hoortoestel onvoldoende de mogelijkheid biedt om spraak te verstaan.

Het doel van dit onderzoek was het evalueren van de relatie tussen intracochleaire elektrische stimulatie (stimulatie in het slakkenhuis) dat gegenereerd wordt door het $\mathrm{Cl}$ en tinnitus. Verder was het doel het optimaliseren van de stimulatiekarakteristieken voor tinnitusonderdrukking.

\section{Samenvatting van de bevindingen}

In Hoofdstuk 1, voerden wij de eerste literatuurstudie uit dat een overzicht laat zien van alle publiekelijk beschikbare artikels over het gebruik van cochleair implantaten als behandeloptie voor tinnitus bij eenzijdig doven. Er werden negen artikels gemeld. Gevonden werd dat het $\mathrm{Cl}$ de tinnitus onderdrukt in het merendeel van de eenzijdig dove patiënten. Sommige studies laten zelfs een complete tinnitus reductie zien na Cl-activatie. Er werd in geen geval een verslechtering van de tinnitus gerapporteerd. Het tinnitus niveau lijkt te stabiliseren na drie tot zes maanden na de eerste fitting. Geconcludeerd wordt dat cochleaire implantatie moet worden beschouwd als een behandeloptie voor tinnitus in eenzijdig doven. Echter, geschikte patiëntselectie lijkt essentieel voor de effectiviteit aangezien verwacht wordt dat voor een positief effect de tinnitus dient te zijn ontstaan door cochleaire deafferentiatie.
Desalniettemin is het bekend dat tinnitus het gevolg kan zijn van de Cl-operatie (complicatie). Echter, de incidentie varieert aanzienlijk tussen de studies. Tot op heden blijft het onduidelijk waarom sommige $\mathrm{Cl}$ patiënten tinnitusklachten ervaren als gevolg van de operatie terwijl andere patiënten deze tinnitusklachten niet ervaren. Een mogelijke verklaring is dat verslechtering van het restgehoor als gevolg van de traumatische insertie van de elektrodedrager de postoperatieve ervaring van tinnitus teweeg kan brengen.

Hoofdstuk 2 beschrijft een retrospectieve studie dat gebruik maakte van de databank van het Cl-team Zuidoost-Nederland, gestationeerd in het Maastricht Universitair Medisch Centrum. Van de 512 patiënten die tussen 2003 en 2013 zijn geïmplanteerd voldeden 247 patiënten aan de inclusiecriteria. Deze 247 patiënten werden een vragenlijst toegezonden waarvan 197 patiënten werden toegelaten in het onderzoek. Er werd geen statistisch significante associatie gevonden tussen de perioperatieve verslechtering van de gehoordrempels (gemeten met behulp van pure toon-audiometrie) en de ontwikkeling of verslechtering van tinnitus. Geconcludeerd wordt dat voor patiënten met preoperatief restgehoor, er geen verhoogd risico op de ontwikkeling van chronische tinnitus na gehoorbeschadiging ten gevolge van cochleaire implantatie lijkt te zijn.

Aangezien het Cleen positief effectlijkt te hebben op tinnitus (hoofdstuk1) rijst de vraag welkmechanisme verantwoordelijk is voor tinnitusonderdrukking na cochleaire implantatie. Is deze onderdrukking te wijten aan de omkering van de veronderstelde ongewenste plasticiteit of is het simpelweg de aandachtsverschuiving van de tinnitus naar omgevingsgeluiden en daardoor minder bewustzijn dat de tinnituswaarneming reduceert?

In Hoofdstuk 3 wordt een prospectieve klinische trial beschreven over een cohort van elf proefpersonen met bilateraal ernstig tot zeer ernstig gehoorverlies, unilateraal $\mathrm{Cl}$ en tinnitus. De meeste proefpersonen waren in staat om kortdurende tinnitus reductie door intracochleaire elektrische stimulatie dat onafhankelijk is van akoestisch geluid te ervaren. Tinnitus kan worden verminderd met hoorbare of zelfs onhoorbare stimuli met een stroomniveau onder de gehoordrempel. Geen duidelijke trends werden gevonden in de optimale stimulatiekenmerken. De optimale stimulatiekenmerken voor tinnitus reductie lijken daarom zeer patiënt-specifiek te zijn.

Om delangetermijneffectenvan intracochleaire elektrischestimulatie datonafhankelijkisvan akoestische input te onderzoeken is een contralateraal normaalhorend oor vereist. Dit contralaterale oor maakt de communicatie mogelijk tijdens intracochleaire elektrische stimulatie dat onafhankelijk is van akoestisch geluid.

Hoofdstuk 4 rapporteert daarom over een patiënt dat zich presenteerde met een slopende tinnitus in het rechter oor dat twee jaar aanwezig was na een idiopathisch plotseling perceptief gehoorverlies in het rechter oor met een gemiddelde gehoordrempel over $0.5,1$ en $2 \mathrm{kHz}$ van $97 \mathrm{~dB}$ hearing level. Zijn rechter oor werd geïmplanteerd met een Cl om de tinnitus te onderdrukken. De standaard klinische Cl-revalidatie werd gevolgd door drie maanden van tinnitus-specifieke intracochleaire elektrische stimulatie (zonder de waarneming van omgevingsgeluiden). Tinnitus kan gedurende maanden worden verminderd met zowel het standaard klinische $\mathrm{Cl}$ als intracochleaire elektrische stimulatie dat onafhankelijk is van een akoestische input. Erwerdgeen negatief effectvan intracochleaire elektrischestimulatie dat onafhankelijk is van een akoestische input op spraakwaarneming in ruis opgemerkt. Bovendien is het aanvullend gebruik van een klinische $\mathrm{Cl}$ voordelig voor spraakdiscriminatie in de gerapporteerde eenzijdig dove patiënt. 
Hoewel de resultaten van hoofdstuk 4 veelbelovend zijn, is het bewijs van deze bevinding gelimiteerd aangezien het een enkele casus betreft. Daarom werd in hoofdstuk 5-7 van dit proefschrift een cohort van tien eenzijdig dove proefpersonen met tinnitus gelokaliseerd in het dove oor beschreven.

Hoofdstuk 5 beschrijft een prospectieve klinische trial over de kortetermijneffecten van tinnitusspecifieke elektrische stimulatie (zonder de waarneming van omgevingsgeluiden) op tinnitus. Bovendien waren met de gebruikte onderzoeksopzet drie aanvullende onderzoeksvragen te beantwoorden: (1) Onderdrukt de $\mathrm{Cl}$-operatie zelf tinnitus? (2) Onderdrukt de klinische $\mathrm{Cl}$ tinnitus? en (3) Is een 1-week durende periode van $\mathrm{Cl}$-deactivatie voldoende om de mogelijke invloed van het verstrekken van een gehoorsensatie op de tinnitus-ervaring uit te sluiten? De resultaten suggereren dat tinnitus kan worden verminderd met tinnitus-specifieke elektrische stimulatie. In sommige gevallen zelfs met onhoorbare stimuli. De optimale stimuli voor tinnitusonderdrukking blijken patiënt-specifiek te zijn. Echter middelluidetotluidestimulionderdrukkentinnitussignificantbeterdanzachte stimuli. Ditkangedeeltelijk worden verklaard door het maskeereffect. Bovendien laat dit hoofdstuk een tinnitus reductie zien tijdens de standaard klinische $\mathrm{Cl}$-revalidatie terwijl de $\mathrm{Cl}$-operatie zelf een positief noch negatief effect op tinnitus heeft. De originele tinnitusluidheid werd hersteld na één week van $\mathrm{Cl}$-deactivatie. Deze bevindingen zijn in lijn met de hypothese dat tinnitus is gerelateerd aan ongewenste neuroplasticiteit als gevolg van gehoorverlies en laten zien dat tinnitus in ieder geval voor minuten kan worden onderdrukt met tinnitus-specifieke intracochleaire elektrische stimulatie.

Het belangrijkste doel van Hoofdstuk 6 is het onderzoeken van de mogelijkheid voor het ontwikkelen van een "Tinnitus Implantaat" (TI), een intracochleaire pulsgenerator voor de onderdrukking van tinnitus. Hoewel de resultaten die verkregen zijn in hoofdstuk 5 veelbelovend lijken te zijn, is langdurige tinnitusonderdrukking een vereiste voor de levensvatbaarheid van het TI. Daarom werden de langetermijneffecten van tinnitus-specifieke elektrische stimulatie (zonder de waarneming van omgevingsgeluiden) vergeleken met de langetermijneffecten van het standaard stimulatiepatroon van een $\mathrm{Cl}$ (met de waarneming van omgevingsgeluiden). Dit hoofdstuk laat zien dat tinnitus ook langdurig kan worden onderdrukt met intracochleaire elektrische stimulatie onafhankelijkvan omgevingsgeluiden. Er werd geen statistisch significant verschil in tinnitusonderdrukking gevonden tussen het standaard klinische $\mathrm{Cl}$ en het experimentele TI.

De codering van omgevingsgeluiden lijkt geen vereiste te zijn voor tinnitusonderdrukking met behulp van intracochleaire elektrische stimulatie. Bovendien blijkt het mogelijk te zijn om tinnitus te onderdrukken met onhoorbare stimuli. Het is daarom aannemelijk dat tinnitusonderdrukking met $\mathrm{Cl}$ niet enkel wordt veroorzaakt door een verschuiving van de aandacht van de tinnitus naar omgevingsgeluiden. Zowel het standaard klinische $\mathrm{Cl}$ als het experimentele TI zijn potentiële behandelopties voor tinnitus. Niettemin, een laatste relevante onderzoeksvraag blijft vooralsnog onbeantwoord, namelijk: Wat is het effect van intracochleaire elektrische stimulatie dat niet codeert voor omgevingsgeluiden, maar wel de tinnitus onderdrukt, op de spraakdiscriminatie bij eenzijdig doven?

In Hoofdstuk 7 werd het effect van tinnitus-specifieke elektrische stimulatie op spraakdiscriminatie gerapporteerd. Bovendien beantwoordden we twee aanvullende onderzoeksvragen: (1) Wat is het effect van het standaard klinische $\mathrm{Cl}$ op spraakdiscriminatie bij eenzijdig doven? en (2) Is formele auditieve training tijdens de $\mathrm{Cl}$-revalidatie in eenzijdig doven voordelig? De resultaten suggereren dat tinnitusspecifieke elektrische stimulatie positieve noch negatieve effecten op spraakdiscriminatie hebben. Het standaard klinische $\mathrm{Cl}$ is bij eenzijdig doven voordelig in zowel spraakdiscriminatie in stilte als in ruis en de subjectief beoordeelde gehoorfunctie. Bovendien lijkt de formele auditieve training niet voordelig te zijn op de spraakdiscriminatie. Echter, het $\mathrm{Cl}$-gerelateerde ongemak tijdens de $\mathrm{Cl}$-revalidatie reduceerde significant sneller bij de proefpersonen die de formele auditieve training ondergingen.

Conclusies en toekomstperspectieven

Dit proefschrift biedt nieuwe en klinisch relevante inzichten in de effecten van intracochleaire elektrische stimulatie op tinnitus. In de verschillende hoofdstukken van dit proefschrift onderdrukt intracochleaire elektrische stimulatie de tinnitus significant. Er werd geen significant verschil gevonden tussen de elektrische stimulatie dat codeert voor omgevingsgeluiden en de elektrische stimulatie dat niet codeert voor omgevingsgeluiden. Bovendien lijken de optimale stimulatiekarakteristieken patiënt-specifiek te zijn. De bevindingen suggereren dat tinnitusonderdrukking mogelijk is door de omkering van de veronderstelde ongewenste plasticiteit. Dit haalbaarheidsonderzoek zal leiden naar de verdere ontwikkeling van intracochleaire elektrische stimulatie als behandeloptie voor tinnitus.

Hoewel er geen bewijs werd gevonden dat stimulatie dat werd afgestemd op de toonhoogte van de tinnitus het optimale stimulatiepatroon is voor tinnitusonderdrukking (hoofdstuk 3 en 5), kan deze behandeloptie mogelijk worden geoptimaliseerd met behulp van Magnetic Resonance Imaging (MRI). Preoperatieve ultra-hoge veldsterkte (functionele) MRI van het centrale zenuwstelsel kan aannemelijk nauwkeuriger en objectief informatie verschaffen over de optimale positie van de intracochleaire stimulatie voor tinnitusonderdrukking in vergelijking met de subjectieve procedure, zoals gebruikt in hoofdstuk 3-5, voor het vinden van de tinnitus toonhoogte. Bovendien zit er mogelijk een discrepantie tussen de waargenomen toonhoogte van de tinnitus en de locatie van de tinnitus binnen de tonotopische organiatie. De tonotopische organisatie van de corticale [Moerel et al., 2014] en subcorticale [De Martino et al., 2013] structuren van de auditieve zenuwbanen kan worden gebruikt om de optimale positie voor stimulatie in de cochlea te voorspellen. Middels postoperatieve Computed Tomography (CT) kan de exacte positie van de afzonderlijke elektroden worden bepaald [Gazibegovic et al., 2014] en kan worden berekend welke elektrode correspondeert met de optimale positie van de stimulatie [unpublished data].

Hoofdstuk 6 van dit proefschrift suggereert dat akoestische informatie geen vereiste is voor tinnitusonderdrukking met intracochleaire elektrische stimulatie. Bovendien lijkt het tot op zekere hoogte mogelijk, met behulp van minder traumatische technieken [Van Abel et al., 2015] het restgehoor te besparen. Toekomstig onderzoek naar het effect van betekenisloze intracochleaire elektrische stimulatie op tinnitus zou daarom mogelijk kunnen worden uitgebreid naar een populatie met aanzienlijk (ipsilateraal) restgehoor. Als alternatief lijkt extracochleaire stimulatie [Di Nardo et al., 2009] een potentiële behandeloptie voor tinnitus in een selectie van de patiënten waarbij behoud van gehoor wenselijk is. Verder kan het zijn dat de effectiviteit van intracochleaire elektrische stimulatie op tinnitusonderdrukking verder wordt geoptimaliseerd door een combinatie van spraakverwerking en de betekenisloze, tinnitus-specifieke elektrische stimulatie [Tyler et al., 2015].

Hoofdstuk 3-6 van dit proefschrift suggereren dat de waargenomen tinnitussuppressie teweeg is gebracht door de omkering van de veronderstelde ongewenste plasticiteit. Deze tinnitussuppressie is niet alleen is te wijten aan een verschuiving van de aandacht van de tinnitus naar omgevingsgeluiden en dus het verminderde bewustzijn. Zowel de frequent waargenomen residuele inhibitie als de mogelijkheid om tinnitus te reduceren met onhoorbare (stimulatie op een niveau onder de gehoordrempel óf stimulatie waarbij luidheidsadaptatie optreedt) elektrische stimulatie ondersteunen deze hypothese. Dit gesuggereerde fysiologische effect in combinatie met de aannemelijke heterogeniteit in de oorzaak van 
tinnitus maken neurostimulatie van de vestibulocochleaire zenuw [Bartels et al., 2007], subcorticale diepe hersenstimulatie [Smit et al., 2015] en corticale stimulatie [Engelhardt et al., 2014] potentiële behandelopties voor tinnitus. Echter, intracochleaire elektrische stimulatie lijkt de voorkeur te hebben door de relatieve eenvoud om toegang te krijgen tot de gewenste structuur, het relatief eenvoudige beloop van de tonotopische organisatie van de cochlea [Greenwood, 1990] en het feit dat de cochlea aan het begin staat van de afferente auditieve zenuwbanen waar de informatie nog niet is doorgestuurd naar beide zijden (ipsilateraal en contralateraal) van de hersenstam, en vervolgens beide hemisferen. Bovendien is het centrale zenuwstelsel gecompliceerder en zijn de verbindingen minder goed begrepen doordat er meerdere parallelle banen zijn.

Hoewel intracochleaire elektrische stimulatie een levensvatbare behandeloptie lijkt te zijn voor tinnitus, is een behandeling op maat vereist voor klinische implicaties waardoor men alle beschikbare potentiële behandelopties moet overwegen. Bij deze overweging zou men eerst moeten kijken naar de minst invasieve behandelopties zoals conventionele hoortoestellen [Hoare et al., 2014], geluidsverrijking met muziek met tinnitus gerelateerde modificaties [Tass et al., 2012] en/of cognitieve gedragstherapie [Jastreboff 1999; Cima et al., 2012].

\section{Referenties}

Bartels H, Staal MJ, Holm AF, Mooij JJ, Alberts FW: Long-term evaluation of treatment of chronic, therapeutically refractory tinnitus by neurostimulation. Stereotact Funct Neurosurg 2007;85(4):150-7.

Cederroth CR, Canlon B, Langguth B: Hearing loss and tinnitus - are funders and industry listening Nature Biotechnology 2013; 31:972-4.

Cima RF, Joore MA, Maes IH, Scheyen DJ, El Refaie A, Baguley DM, Vlaeyen JW, Anteunis LJ: Costeffectiveness of multidisciplinary management of tinnitus at a specialized Tinnitus centre. BMC Health Serv Res 2009;11;9:29.

Cima RF, Maes IH, Joore MA, Scheyen DJ, El Refaie A, Baguley DM, Anteunis LJ, van Breukelen GJ, Vlaeyen JW: Specialised treatment based on cognitive behaviour therapy versus usual care for tinnitus: randomized controlled trial. Lancet 2012;379(9830):1951-9.

Davis PB, Paki B, Hanley PJ: Neuromonics tinnitus treatment: third clinical trial. Ear Hear 2007;28:242259

De Martino F, Moerel M, van de Moortele PF, Ugurbil K, Goebel R, Yacoub E, Formisano E: Spatial organization of frequency preference and selectivity in the human inferior colliculus. Nat Commun 2013;4:1386.

Di Nardo W, Cianfrone F, Scorpecci A, Cantone I, Giannantonio S, Paludetti G: Transtympanic electrical stimulation for immediate and long-term tinnitus suppression. Int Tinnitus J 2009;15(1):100-6.

Du Verney G: Traité de l'organe de l'ouïe: contentant la structure, les usages \& les maladies de toutes les parties de l'oreille (1731). Leide: Joh. Arn. Langerak.

Engelhardt J, Dauman R, Arné P, Allard M, Dauman N, Branchard O, Perez P, Germain C, Caire F, Bonnard $D$, Cuny E: Effect of chronic cortical stimulation on chronic severe tinnitus: a prospective randomized double-blind cross-over trial and long-term follow up. Brain Stimul 2014;7(5):694-700.

Engineer ND, Riley JR, Seale JD, Vrana WA, Shetake JA, Sudanagunta SP, Borland MS, Kilgard MP: Reversing pathological neural activity using targeted plasticity. Nature 2011;470(7332):101-104.

Erlandsson S, Ringdahl A, Hutchins T, Carlsson SG: Treatment of tinnitus: a controlled comparison of masking and placebo. Br J Audiol 1987;21(2):37-44.

Fioretti A, Peri G, Eibenstein A: Suppression of tinnitus in a patient with unilateral sudden hearing loss: a case report. Case Reports in Otolaryngology DOI: 10.1155/2012/210707.

Gazibegovic D, Dees G, van Hoof M, Stokroos R: The assessment of the scalar positionof the HiFocus Mis Scala electrode based on MRI and Cone Beam CT fusion. $8^{\text {th }}$ International symposium on objective measures in auditory implants 2014; http://www.epostersonline.com/omai2014/node/202 
Greenwood DD: A cochlear frequency-position function for several species - 29 years later. J Acoust Soc Am 1990;87(6):2592-2605.

Hoare DJ, Edmondson-Jones M, Sereda M, Akeroyd MA, Hall D: Amplification with hearing aids for patients with tinnitus and co-existing hearing loss. Cochrane Database of Systematic Reviews DOI: 10.1002/14651858.CD010151.pub2.

Jastreboff PJ: Tinnitus retraining therapy. Br J Audiol 1999;33(1):68-70

Moerel M, De Martino F, Formisano E: An anatomical and functional topography of human auditory cortical areas. Front Neurosci 2014;8:225.

Pantev C, Okamoto H, Teismann H: Music-induced cortical plasticity and lateral inhibition in the human auditory auditory cortex as foundations for tonal tinnitus treatment. Front Syst Neurosci DOI:

$10.3389 / 2012 / 00050$

Pantev C, Okamoto H, Teismann H: Tinnitus: the dark side of auditory cortex plasticity. Ann NY Acad Sci 2012;1252:253-258

Reavis KM, Rothholtz VS, Tang Q, Carroll JA, Djalilian H, Zeng F-G: Temporary suppression of tinnitus by modulated sounds. J Assoc Res Otolaryngol 2012;13(4):561-71.

Saltzman M, Ersner M: A hearing aid for the relief of tinnitus aurium. Laryngoscope 1947;57:358-66.

Shekhawat GS, Searchfield GD, Stinear CM: Role of hearing aids in tinnitus intervention: a scoping review. J Am Acad Audiol 2013;24:747-762.

Smit JV, Janssen ML, Schulze H, Jahanshahi A, Van Overbeeke JJ, Temel Y, Stokroos RJ: Deep brain stimulation in tinnitus: current and future perspectives. Brain Res 2015;1608:51-65.

Tass PA, Adamchic I, Freund H-J, von Stackelberg T, Hauptmann C: Counteracting tinnitus by acoustic coordinated reset neuromodulation. Restor Neurol Neurosci 2012;30:137-159.

Turner O, Windfuhr K, Kapur N: Suicide in deaf populations: a literature review. Ann Gen Psychiatry 2007;6:26.

Tyler RS, Keiner AJ, Walker K, Deshpande AK, Witt S, Killian M, Ji H, Patrick J, Dillier N, van Dijk P, Lai WK, Hansen MR, Gantz B: A series of case studies of tinnitus suppression with mixed background stimuli in a cochlear implant. Am J Audiol 2015; [Epub ahead of print]

Van Abel KM, Dunn CC, Sladen DP, Oleson JJ, Beatty CW, Neff BA, Hansen M, Gantz BJ, Driscoll CL:

Hearing preservation among patients undergoing cochlear implantation. Otol Neurotol 2015;36(3):416 21.

Vernon JA, Meikle MB: Masking devices and alprazolam treatment for tinnitus. Otolaryngol Clin N Am 2003;36:307-320 
Het is gelukt: mijn boekje is af! Nu is het tijd om terug te kijken op een fantastische tijd waarin ik veel heb mogen leren en een aantal bijzondere mensen heb ontmoet. Een aantal mensen wil ik graag persoonlijk bedanken.

Allereerst en vooral wil ik mijn promotor professor dr. Robert Stokroos bedanken. Beste Robert, bedankt dat jij mij 4 jaar geleden het vertrouwen gaf. Dit promotietraject heeft pieken en dalen gekend, maar het enthousiasme en doorzettingsvermogen heeft ons gebracht waar we nu staan. Ik vond het zeer prettig om onafhankelijk te werken terwijl ik wist dat ik altijd op je kon terugvallen wanneer dit noodzakelijk was. Deze vrijheid gaf mij de ruimte om extra onderzoek te verrichten. Ik ben er trots op dat ik samen met dit team heb mogen werken. Bedankt voor alles wat ik tijdens dit promotietraject heb mogen leren!

Geachte dr. Erwin George, beste Erwin, wij mochten nagenoeg gelijktijdig beginnen met onze werkzaamheden in het MUMC+. Tijdens dit roerige begin hebben wij elkaar gevonden. Ik ben er trots op dat ik de eerste doctor zal worden die jij aflevert aan de samenleving. Eén ding weet ik zeker; er zullen er nog velen volgen. Ik wil je via deze weg bedanken voor hetgeen ik de afgelopen 4 jaar geleerd heb, je onbegrensde toegankelijkheid en de mooie momenten waaronder de bijscholing in Innsbruck en het congres in Ann Arbor. Jouw citaat "promoveren is het minimaliseren van onafhankelijkheden" ben ik over de jaren steeds meer gaan begrijpen.

Geachte leden van de promotiecommissie: professor dr. Bernd Kremer, professor dr. Pim van Dijk, professor dr. Werner Mess, professor dr. Yasin Temel en dr. Katrien Vermeire, hartelijk dank voor uw tijd, expertise en bereidwilligheid om een deskundig oordeel te geven over dit proefschrift. Beste Katrien, voor jou speciale dank voor onze samenwerking aan het begin van dit project. Jouw ervaring was essentieel in de totstandkoming van het onderzoeksprotocol. Bovendien heb ik hoofdstuk 1 van dit proefschrift samen met jou mogen schrijven. Ik ben blij dat jij als lid van de leescommissie een officiële rol bij mijn verdediging krijgt.

Dear Andreas Griessner PhD, dear Andi, at the start of this PhD program I have met you twice; once in Innsbruck and once in Maastricht. These visits have laid the foundation for our intensive contact by email. Thank you for your help in solving technical problems and your critical revisions on our manuscripts. You made me grow in my work, and I'm deeply grateful for that.

Verder gaat mij dank uit naar alle patiënten die hun bijdrage hebben geleverd aan de totstandkoming van dit proefschrift. Zonder de geschikte proefpersonen was het niet gelukt om klinisch wetenschappelij onderzoek te verrichten. In het bijzonder wil ik de proefpersonen bedanken die gedurende ruim anderhalf jaar veelvuldig naar Maastricht zijn afgereisd voor het verzamelen van de onderzoeksgegevens. Ik wil jullie bedanken voor de enorme bereidwilligheid en flexibiliteit gedurende deze periode. Ik heb jullie persoonlijk leren kennen waarbij mooie maar soms ook droevige gebeurtenissen werden gedeeld. Jullie gaven mijn onderzoek kleur waaruit ik de energie haalde om dit project tot een succes te maken.

De leden van de Data Safety Monitoring Board; drs. Jan Wouter Brunings, drs. Fons Kessels en drs. Dyon Scheijen en de onafhankelijk arts; dr. Kenneth Kross wil ik bedanken voor de belangeloze samenwerking. Zonder jullie medewerking hadden een aantal onderzoeken niet mogen starten.

Voor de statistische hulp wil ik graag dr. Mickey Chenault en later drs. Miranda Janssen bedanken. Ik heb jullie hulp bij mijn statistische uitdagingen als een luxe ervaren. Beste Mickey, na het behalen van je PhD mag eindelijk gaan genieten van je pensioen. Ik wens je daar heel veel plezier van.
Bij wetenschappelijk onderzoek behoren ook monitors en audits. Als monitor wil ik dr. Marijke Buckx bedanken voor haar inspanning bij het controleren van mijn administratie. Als tijdelijke kamergenoot wi ik je ook bedanken voor je interesse in mijn onderzoek.

De collega's van het Audiologisch Centrum, hartelijk dank voor jullie interesse in mijn onderzoek. Jullie teamspirit zorgde ervoor dat ik mij al snel thuis voelde. Jullie flexibiliteit en enthousiasme zal ik niet sne vergeten. Speciale dank voor dr. Lucien Anteunis voor alle kennis en wijsheden die je op mij overbracht en Mirçea Rikers voor alle zaken met betrekking tot de Cl-onderdelen. Verder zijn er drie dames van essentieel belang geweest bij dit proefschrift. Drs. Nadia Hendrice, jij informeerde mij in 2007 met een college over het bestaan van cochleair implantaten. Dit fascinerende college heeft de keuze voor mijn masteropleiding beïnvloed. Tijdens deze masteropleiding was jij degene die mij met mijn verzoek voo een eindstage over cochleair implantaten in contact bracht met professor Robert Stokroos. Na een sollicitatiegesprek en een uitgebreide literatuurstudie heb ik onder dagelijkse supervisie van drs. Winde Rerren en drs. Joke Debruyne een begin gemaakt aan wat uiteindelijk heeftgeresulteerd in dit proefschrift. Nogmaals hartelijk dank voor jullie bijdrage hieraan en Winde en Joke, veel plezier van jullie kersverse gezin!

De stafleden en arts-assistenten wil ik bedanken voor de interesse in en medewerking aan mijn onderzoek. Ik heb de samenwerking als zeer prettig ervaren. Verder zijn een aantal van jullie mij gedurende dit traject voorgegaan in het behalen van zijn of haar academische graad tot doctor. De ervaring die ik daarbij heb opgedaan zal ongetwijfeld van pas komen.

Verder wil ik Edith Berry, de 'poli-dames' en de secretaresses van de KNO-afdeling en het Audiologisch Centrum bedanken met in het bijzonder Jacqueline Hageman die het voor mij mogelijk maakte alle patiënten op het juiste moment in de juiste behandelkamers te zien. Verder speciale dank voor Marèse Gordijn voor het plannen van interne afspraken en de begeleiding bij administratieve zaken. Zonder jullie zou dit proefschrift meerdere malen zijn gestrand. Bedankt voor jullie organisatie en geduld.

Beste drs. Elke Devocht en drs. Marc van Hoof, jammer dat het niet mogelijk is om je door meer dan twe paranimfen terzijde te laten staan... Ik ben ervan overtuigd dat de vele discussies en gedachteexperimenten vanuit onze verschillende achtergronden ten goede zijn gekomen aan de kwaliteit van onze onderzoeken. We zaten alle drie op het 'CTCM-eilandje' waardoor we met dezelfde perikelen kampten. Dagelijks gingen we lunchen en eens in de twee weken hadden wij onze 'boekclub'. Dit heeft uiteindelijk geleid tot een gezamenlijk manuscript dat aanzienlijk meer inspanning heeft gekost dan da vooraf werd ingeschat. Ook dat is onderzoek! Marc, met jou deelde ik zelfs 3 jaar een kamer en gingen we reregeld squashen in het ziekenhuis. Het aantal cappuccino's dat wij samen dronken is ontelbaar. Ik heb mijn tijd met jullie als zeer prettig ervaren en hoop dat we in de toekomst contact zullen houden. Bedankt dat jullie mijn klankbord wilden zijn en ik wens jullie heel veel succes met de afronding van jullie eigen promotie.

Mijn collega-onderzoekers dr. Anouk Linssen, drs. Gusta van Zwieten en bovendien kamergenoten drs. Tim Calon, drs. Michel van Hooren en in het eerste jaar drs. Lindsay de Rozario wil ik bedanken voor de prettige tijd samen. Het delen van ervaringen levert wederzijds veel winst op! Ik vond het fijn om jullie beter te leren kennen en wil jullie heel veel succes wensen bij jullie onderzoek, opleiding en verdere carrière. 
Gedurende de afgelopen jaren heb ik ook een aantal stagiairs/stagiaires mogen begeleiden. Ik wil Tilmann Netz, Bart Pielkenrood en Fabienne Dohmen bedanken voor de prettige samenwerking en jullie enthousiasme in hetverrichtenvan wetenschappelijkonderzoek. Ikbenertrotsop datonzesamenwerking heeft geresulteerd in manuscripten die voor publicatie zijn aangeboden.

From the Cognitive Neuroscience group (FPN) I want to thank Professor Elia Formisano PhD, Federico De Martino PhD, Lars Riecke PhD, Bettina Sorger PhD and Faruk Gülban MSc. It is a pleasure to work with you on the ongoing ultra-high field MRI projects. Special thanks to Federico for the intensive cooperation.

Drs. Kevin Hermans en drs. Brecht Willems, hoewel we alle drie compleet verschillende onderzoeken deden zaten we doordat we nagenoeg gelijk begonnen aan een promotietraject in hetzelfde schuitje. Wat was het geruststellend om af en toe de bevestiging te krijgen dat onderzoek nu eenmaal gepaard gaat met tegenslagen. Gelukkig hadden we ook onze succesjes. Bedankt voor de gezellige lunches en diners!

Ik wil ook mijn nieuwe collega's bij TFS bedanken voor de goede samenwerking en prettige sfeer. Ik voe me al vanaf de eerste week bij jullie thuis en ik ben ervan overtuigd dat we een hele mooie tijd tegemoet gaan.

Bij fulltime onderzoek (lees: onderzoek waarmee je opstaat en naar bed gaat) is het belangrijk om te kunnen relativeren. Daarom wil ik mijn vrienden bedanken voor de welkome afleiding onder het genot van een hapje en/of een drankje, de feestjes, festivals of zoals afgelopen zomer de vakantie in Lagos. Ook al zie ik de meeste van jullie minder vaak dan in onze studententijd, jullie vriendschap is heel belangrijk voor me. Bovendien wil ik van de gelegenheid gebruik maken om mij te excuseren voor de keren dat ik op het laatste moment moest afzeggen omdat ik weer eens een deadline moest halen;-).

Een speciaal woord voor mijn paranimfen Clifton Didden en drs. Jasper Smit. Clifton, we hebben elkaar leren kennen via een bijbaantje wat resulteerde in een hechte vriendschap. Ik ben blij dat wij elkaar ondanks de afstand nog met enige regelmaat zien en hoop dat dit zo zal blijven. Jasper, jij startte niet veel later dan ik aan je promotieonderzoek naar tinnitus. Doordat we vergelijkbaar onderzoek deden zijn we samen naar symposia en congressen geweest. Bij ons eerste congres in Brugge was ik mijn tandenborste vergeten en jij bood aan om jouw tandenborsten te lenen. Ondanks dat ik nog een nieuwe kon kopen was dat het begin van een prettige samenwerking. We hebben samen onderzoek verricht en na ons laatste congres reisden we zelfs samen met Jeanny en Marieke door Canada en de VS. Ik wens je heel veel succes bij je opleiding en de afronding van je promotie. Hopelijk blijven wij contact houden. Ik zal bij mijn verdediging ongetwijfeld blij zijn met jullie aan mijn zijde! Bedankt dat jullie mijn paranimf willen zijn.

Naast mijn vrienden wil ik ook mijn familie bedanken voor alle steun. Pap en mam, dank jullie wel voor jullie onvoorwaardelijk steun, liefde, goede zorgen en jullie absolute vertrouwen in mij. Ik kan altijd bij jullie terecht. Jullie zijn mijn stabiele basis en leerden mij om van het leven te genieten. Ik ontspan volledig zodra ik bij jullie thuis kom en ikvind het altijd heerlijk om bij jullie te zijn. Bedankt voor alle mogelijkheden die jullie mij hebben geboden, maar ook voor alle levenslessen, warmte, advies, uitstapjes, vakanties en etentjes. Kim en Davy, dank jullie wel voor jullie interesse in mij als persoon. Ik ben trots op jullie, kijk er elke keer naar uit om jullie en de kinderen te zien en ben heel blij dat onze band zo goed is.

Mijn 'schoonfamilie' wil ik bedanken voor alle steun en vertrouwen. Leo, Mechtild, Geert, Marjolein, Roel en Annemijn, bedankt voor alle leuke momenten samen. Ik heb het met jullie getroffen!
Lieve Marieke, hetlaatste woord richtikaan jou. Wat ben jij belangrijk geweest bijde totstandkoming van dit proefschrift, maar nog veel meer voor mij als persoon! Ik hoop dat ik nog heel lang van je mag genieten Altijd heb je mij gesteund en gestimuleerd en nooit heb je geklaagd als ik weer eens achter mijn laptop moest. Ik vind het heerlijk om met jou te gaan uiteten, weekendjes weg te gaan, op vakantie te gaan of's avonds met en een glas wijn op de bank te ploffen. Bedankt voor alles! 
Remo Arts is geboren op 6 februari 1987 te Heerlen waar hij zijn gehele jeugd heeft doorgebracht. Van 1999 tot en met 2005 heeft hij in Heerlen op het Sintermeertencollege het VWO gevolgd (profiel "Natuur \& Gezondheid" met als bijvakken Natuurkunde2,

Scheikunde2 en Kwantummechanica). In 2005 werd gestart met de bacheloropleiding

Moleculaire LevensWetenschappen aan de universiteit van Maastricht (NL). Deze opleiding werd in 2009 succesvol afgerond waarna van 2009 tot en met 2011 de masteropleiding BioElektronica en Nanotechnologie aan de universiteit van Hasselt (BE) werd gevolgd. Deze opleiding werd tussentijds bezegeld met een grote onderscheiding en de gehele opleiding resulteerde in een onderscheiding. De volgende aanvullende certificaten werden behaald: Opleiding Stralingsbescherming, Proefdierkunde (FELASA B) en Nanobiotechnologie.

Tijdens zijn masteropleiding kwam Remo in aanraking met actieve implantaten waaronder cochleaire implantaten. Zijn interesse voor dit bionische oor resulteerde in een eindstage bij de afdeling KNO in het academisch ziekenhuis Maastricht (MUMC+). Een vervolg aan deze stage werd gegeven middels een promotieonderzoek waarvan dit proefschrift het resultaat is. Naast zijn primaire werk was Remo actief in wetenschappelijke projecten aangaande ultra-hoge veldsterkte (7 Tesla) (functionele) MRI van corticale en subcorticale auditieve structuren bij tinnitus en het optimaliseren van van de $\mathrm{Cl}$-filterbanken midde 3 dimensionale beeldvormi Zaltbommel. 
Smit JV, Pielkenrood BJ, Arts RAGJ, Stokroos RJ: Patient acceptance of novel invasive treatments for tinnitus. Submitted for publication.

Dohmen FYM*, Arts RAGJ*, George ELJ, Stokroos RJ:. Mechanisms of loudness adaptation in electric hearing. Submitted for publication.

Arts RAGJ, George, ELJ, Janssen AML, Griessner A, Zierhofer C, Stokroos RJ: Hearing after cochlear implantation in patients with unilateral severe to profound hearing loss accompanied with tinnitus and the effect of formal auditory training. Submitted for publication.

Arts RAGJ, George ELJ, Janssen AML, Griessner A, Zierhofer C, Stokroos RJ: Tinnitus suppression by intracochlear electrical stimulation in single sided deafness - a prospective clinical trial: Part II. Submitted for publication.

Arts RAGJ, Netz T, Janssen AML, George ELJ, Stokroos RJ: The occurrence of tinnitus after Cl surgery in patients with severe hearing loss: A retrospective study. Int J Audiol. 2015;Sep24:1-8. [Epub ahead of print]

Devocht EMJ, Dees G, Arts RAGJ, Smits JJ, George ELJ, van Hoof M, Stokroos RJ: Revisiting place-pitch match in Cl listeners using 3D imaging analysis. Ann Oto Rhinol Laryn. Accepted.

Arts RAGJ, George ELJ, Griessner A, Zierhofer C, Stokroos RJ: Long-term effects of intracochlear electrical stimulation with looped patterns on tinnitus: a case report. ENT J. Accepted.

Arts RAGJ, George ELJ, Griessner A, Zierhofer C, Stokroos RJ: Tinnitus suppression by intracochlear electrical stimulation in single sided deafness - a prospective clinical trial: Part I. Audiol Neurotol 2015;20:294-313.

Arts RAGJ, George ELJ, Chenault MN, Stokroos RJ: Optimizing intracochlear electrical stimulation to suppress tinnitus. Ear Hear 2015;36:125-135.

Arts RAGJ, George ELJ, Stokroos RJ, Vermeire K: Review: cochlear implants as a treatment of tinnitus in single sided deafness. Curr Opin Otolaryngol Head Neck Surg 2012;20:398-403. 


\section{The Illustrated Guide to a Ph.D. by Matt Might}

Imagine a circle that contains all of human knowledge:

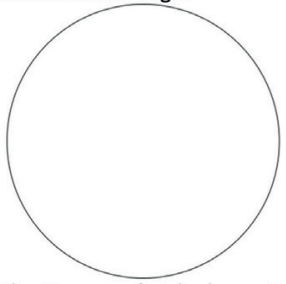

By the time you finish elementary school, you know a little:

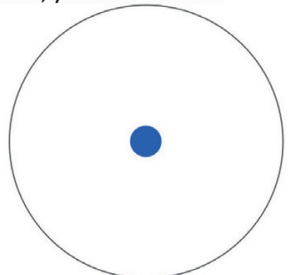

By the time you finish high school, you know a bit more:

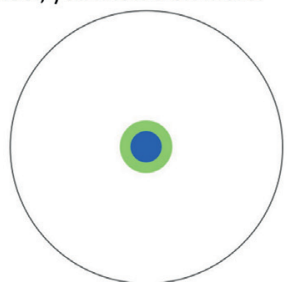

With a bachelor's degree, you gain a specialty:

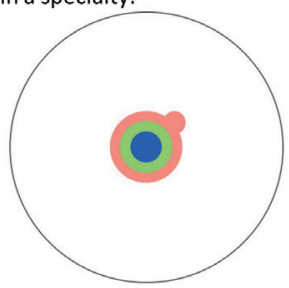

A master's degree deepens that specialty:

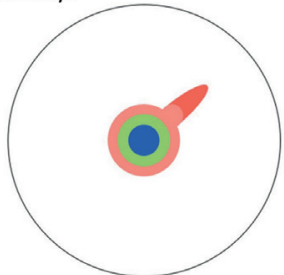

Reading research papers takes you to the edge of human knowledge:

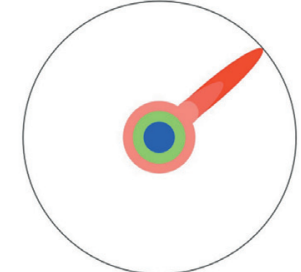

Once you're at the boundary, you focus:

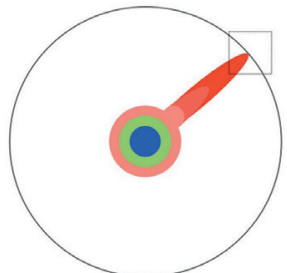

You push at the boundary for a few years:

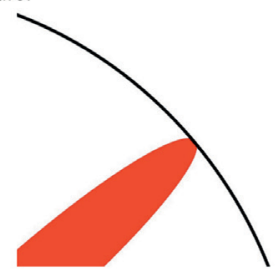

Until one day, the boundary gives way:

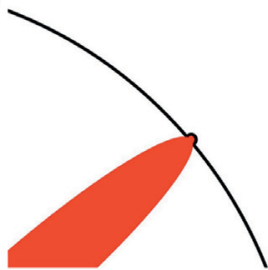

And, that dent you've made is called a Ph.D.:

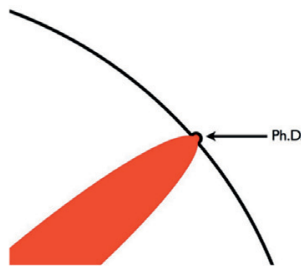

Of course, the world looks different to you now:

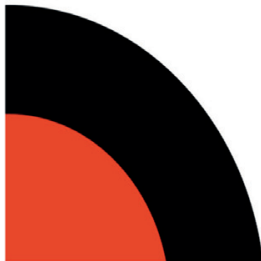

So, don't forget the bigger picture:

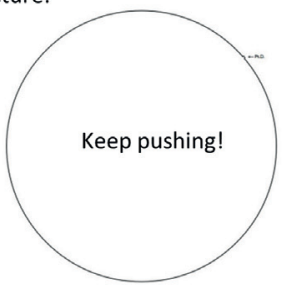

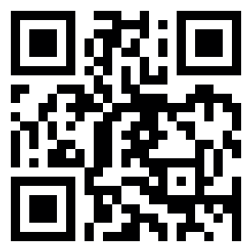

